\title{
ALMENO UNA STELLA \\ Un progetto di tutoraggio per gli adolescenti immigrati
}

a cura di Graziella Favaro e Monica Napoli

\section{La melagrana}

Ricerche e progetti per l'intercultura

FrancoAngeli open 2 ACCESS 


\section{La melagrana}

\section{Collana diretta da Graziella Favaro e Massimiliano Fiorucci}

La collana La melagrana articola la sua proposta editoriale su due diversi piani dell'educazione interculturale: le idee e le pratiche.

La sezione Idee e metodi propone contributi teorici, riflessioni e materiali che offrono spunti da sviluppare nel lavoro interculturale.

La sezione Ricerche e progetti descrive e commenta esperienze e progetti realizzati, con uno sguardo attento al significato generale che possono avere anche in situazioni diverse da quelle in cui sono nati.

In ogni caso l'attenzione è rivolta a proporre dei testi che mettano in luce temi e problemi sinora poco sviluppati nell'ambito della pubblicistica sull'educazione

interculturale e che sappiano integrare i due piani che abbiamo indicato.

I lettori a cui è dedicata questa collana sono soprattutto gli insegnanti in formazione $o$ in servizio, ma i testi si rivolgono anche agli operatori dei servizi sociali, alle educatrici degli asili nido, alle figure di mediazione interculturale che non svolgono il loro lavoro nella scuola.

Questo anche nella convinzione che un efficace lavoro interculturale possa svilupparsi solo attraverso la collaborazione tra la scuola e le istituzioni

formative del territorio e con un contatto tra tutte le figure professionali che operano nei diversi ambiti.

\section{Comitato SCiEnTifico}

Ivana Bolognesi, Università di Bologna Marco Catarci, Università di Roma Tre Cristina Allemann-Ghionda, Università di Colonia Elio Gilberto Bettinelli, Università di Milano-Bicocca Giovanna Campani, Università di Firenze Don Virginio Colmegna, Fondazione Casa della Carità

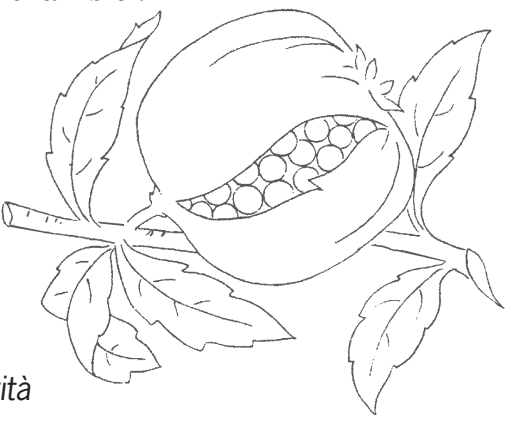
Duccio Demetrio, Università di Milano-Bicocca

F. Javier García Castaño, Università di Granada

Antonio Genovese, Università di Bologna

Francesca Gobbo, Università di Torino Jahdish Gundara, Università di Londra Lorenzo Luatti, Ucodep - Centro di Documentazione Città di Arezzo

Raffaele Mantegazza, Università di Milano-Bicocca

Giuseppe Milan, Università di Padova

Marie Rose Moro, Università di Paris Descartes

Vinicio Ongini, esperto Miur

Agostino Portera, Università di Verona

Milena Santerini, Università Cattolica del Sacro Cuore, Milano

Clara Silva, Università di Firenze

Massimiliano Tarozzi, Università di Bologna

Maria Sebastiana Tomarchio, Università di Catania

Alessandro Vaccarelli, Università dell'Aquila

Davide Zoletto, Università di Udine

Tutti i volumi pubblicati sono sottoposti a referaggio in "doppio cieco". Il Comitato scientifico può svolgere anche le funzioni di Comitato dei referee. 

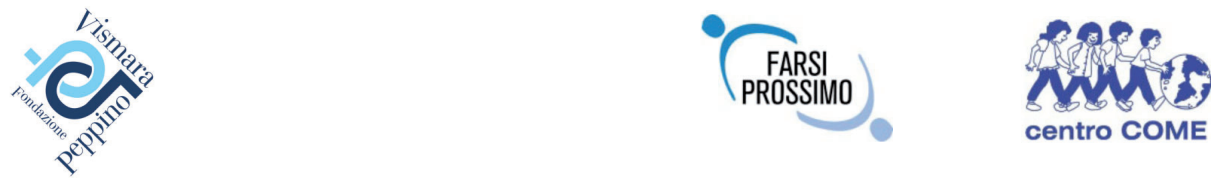

Il progetto "Almeno una stella" è stato promosso e sostenuto dalla Fondazione "Vismara" di Milano, e realizzato dal Centro COME della cooperativa "Farsi Prossimo", in collaborazione con i partner e le Università dei territori coinvolti.

Gruppo di progetto

Graziella Favaro, ideazione e direzione scientifica

Monica Napoli, responsabilità del progetto

- Milano - Centro COME, Cooperativa Farsi Prossimo

Michela Casorati, coordinamento delle scuole Sara Tesco, coordinamento dei tutor

- Arezzo - Centro di documentazione città di Arezzo; Oxfam Italia

Lorenzo Luatti, referente di progetto

Elisa Carboni, Francesca Terenzi, coordinamento di progetto

- Bologna - CD-LEI Comune di Bologna - Settore Istruzione; cooperativa CIOFS FP

Raffaella Pagani, referente di progetto Comune di Bologna Horst Wiedemann, coordinamento didattico Laura Belvedere, referente di progetto CIOFS

- Torino - Centro Interculturale città di Torino; Associazione ASAI

Anna Ferrero, referente di progetto

Alessia Marchiò, Alice Turra, coordinamento didattico

Karima Ben Salah, coordinamento dei tutor

- Provincia di Trento - Centro Interculturale "Millevoci"

Laura Bampi, referente di progetto

Leila Ziglio, Maria Arici, coordinamento delle azioni

- Rete scuole della Regione Friuli

Sandra Romanin e Flavia Virgilio, referenti del progetto

Maria Pisani, Flavia Virgilio, coordinamento delle azioni
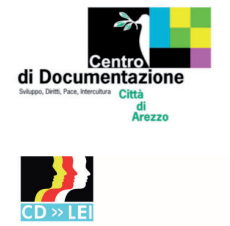

Centro di Documentazione
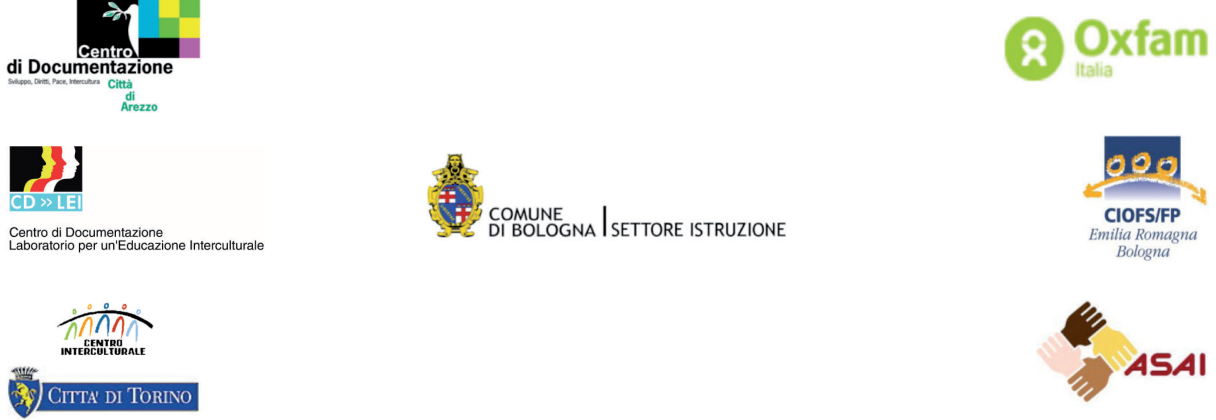

DI BOLOGNA SETTORE ISTRUZIONE

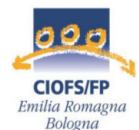




\section{ALMENO UNA STELLA Un progetto di tutoraggio per gli adolescenti immigrati}

a cura di Graziella Favaro e Monica Napoli

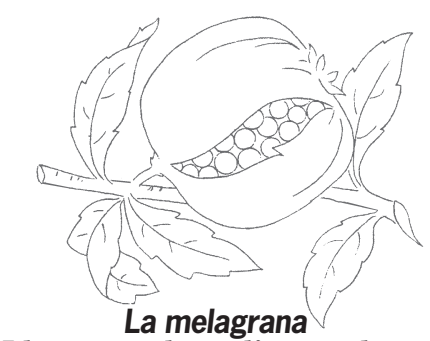

Idee e metodi per l'intercultura

FrancoAngeli 
Graziella Favaro si occupa da tempo dei processi educativi nella migrazione. Sui temi dell'inclusione scolastica, dell'italiano come seconda lingua, dell'educazione interculturale svolge un lavoro di ricerca, formazione, progettazione ed elaborazione di materiali. Fa parte dell'Osservatorio sull'integrazione degli alunni stranieri e l'intercultura del MIUR. Dirige la rivista per la scuola multiculturale "Sesamonline" e la collana "La melagrana. Idee e metodi per l'intercultura". Fra i suoi testi: A scuola nessuno è straniero. Insegnare e apprendere nella scuola multiculturale (2014); Dare parole al mondo (2012); Insieme in italiano (2016).

Monica Napoli si occupa di progettazione territoriale, in particolare in ambito interculturale. Ha coordinato il Centro COME della cooperativa Farsi Prossimo di Milano ed attualmente è responsabile della progettazione per la stessa cooperativa. Ha coordinato numerosi progetti per l'integrazione dei minori e delle famiglie straniere, soprattutto in ambito scolastico e socio-educativo. Ha curato: Come un pesce fuor d'acqua. Il disagio nascosto dei bambini e dei ragazzi immigrati (con G. Favaro, 2002); L'inserimento scolastico dei bambini rom e sinti (con S. Ignazi, 2004); Ragazze e ragazzi nella migrazione. Adolescenti stranieri: identità, raccon$t i$, progetti (con G. Favaro, 2004).

Copyright @ 2016 by FrancoAngeli s.r.1., Milano, Italy

L'opera, comprese tutte le sue parti, è tutelata dalla legge sul diritto d'autore ed è pubblicata in versione digitale con licenza Creative Commons Attribuzione-Non Commerciale-Non opere derivate 3.0 Italia (CC-BY-NC-ND 3.0 IT)

L'Utente nel momento in cui effettua il download dell'opera accetta tutte le condizioni della licenza d'uso dell'opera previste e comunicate sul sito

http://creativecommons.org/licenses/by-nc-nd/3.0/it/legalcode 


\section{Indice}

Presentazione, Fondazione "Peppino Vismara"

pag. $\quad 9$

Introduzione, di Graziella Favaro

1. Uno sguardo alla scuola multiculturale e ai suoi protagonisti, di Graziella Favaro

1. Due priorità, un "modello asistematico"

2. Bisogni educativi specifici o speciali?

3. Tra ostacoli e attese

4. L'italiano di scolarità: la tigre sul cammino

5. I fattori collegati al rischio della dispersione scolastica

6. I più piccoli, i più grandi

Riferimenti bibliografici

2. II progetto e le azioni nelle città, di Michela Casorati e Monica Napoli

1. A partire dalle "Bussole"... Alle origini del progetto

2. Molte presenze, diversi Paesi

3. Gli obiettivi di "Almeno una stella"

4. Quattro territori più due

5. Il modello organizzativo

Riferimenti bibliografici e sitografia

3. Ricominciare da capo. Adolescenti che migrano e inserimento scolastico, di Graziella Favaro

1. Ragazzi che attraversano i confini

2. I volti e le storie

3. Il viaggio all'improvviso 
4. Le fatiche della migrazione

pag. 47

5. Uno spazio famigliare ristretto

6. "All'inizio non capivo niente" $\gg 51$

7. Farsi dei nuovi amici $\gg 54$

8. Far fronte a episodi di discriminazione $\gg 56$

9. Immaginare il futuro $\gg 58$

10. Orientati o disorientati?

11. Fattori in gioco al momento delle scelte $\quad$ » 61

12. I "quasi adatti" 》 64

Riferimenti bibliografici $\gg 66$

4. Ritratti e percorsi scolastici dei ragazzi accompagnati, di Michela Casorati e Monica Napoli 》 68

1. Le caratteristiche dei ragazzi $» 68$

2. Le situazioni scolastiche e linguistiche 》 70

3. Gli interventi realizzati nel primo anno » 72

4. Gli esiti alla fine del primo anno » 74

5. Gli interventi realizzati nel secondo anno » 79

6. Gli esiti alla fine del secondo anno » 83

7. L'apprendimento dell'italiano » 85

8. Alcune considerazioni finali » 87

5. Stare accanto. Modi e figure del tutoraggio, di Graziella Favaro

1. "Stelle" sul cammino

2. Tra vicinanza e giuste distanze

3. Specchi e finestre

4. Ritratti di tutor

5. I punti di forza del tutoraggio

6. Modelli diversi nelle città

7. In ascolto delle storie 103

8. Le fasi del tutoraggio 105

9. La bellezza della responsabilità

10. I diversi volti del tutoraggio

Riferimenti bibliografici

Allegato 1. Il "diario di bordo" di S.

6. Un compagno, una "spalla", un martello. I modi diversi di essere tutor, di Graziella Favaro, Alessia Marchiò, Horst Wiedemann, Leila Ziglio, Francesca Terenzi e Flavia Virgilio

1. Un prisma a più facce, di Graziella Favaro 
2. Tutoring e cittadinanza: scoprire la città insieme, di Alessia Marchiò

3. Gli aspetti relazionali del tutoring scolastico, di Horst Wiedemann

4. Costruire la resilienza attraverso il tutoring, di Leila Ziglio

5. I diversi stili dei tutor, di Francesca Terenzi

6. "Almeno una stella" in Friuli Venezia Giulia: la scuola oltre la scuola, di Flavia Virgilio

7. Vademecum per un progetto di tutoraggio. Linee guida, obiettivi, strumenti, di Graziella Favaro

Premessa

1. Reperire i tutor

2. Formare i tutor

172

3. Riconoscere l'esperienza di tutoraggio

4. Individuare gli alunni da accompagnare e stabilire accordi con le scuole

5. Stabilire un "patto di tutoraggio" fra i soggetti coinvolti

6. Definire e gestire le quattro fasi del progetto

7. Curare le relazioni, in presenza e a distanza

177

8. Monitorare e supportare le azioni di tutoraggio

178

9. Gestire la fase di chiusura del progetto

10. Valutare, documentare, condividere

178

179

Allegato 1. Esempio di presentazione del progetto lano/corso di laurea in Mediazione linguistica e culturale

Allegato 5. Scheda/tipo sullo studente e sul percorso scolastico

Allegato 6. Patto di tutoraggio

Allegato 7. Scheda per l'osservazione delle caratteristiche di resilienza dei ragazzi

Allegato 8. Questionario sulla conoscenza della città

Allegato 10. Questionario sulla valutazione dell'esperienza di tutoraggio da parte dei ragazzi accompagnati 



\section{Presentazione}

La Fondazione "Peppino Vismara" di Milano sostiene fin dalle sue origini interventi a carattere sociale, culturale o educativo rivolti ai più fragili e bisognosi, in Italia e nei paesi in via di sviluppo. Un'attenzione particolare è rivolta ai più giovani, e a progetti che, oltre a sostenerne la crescita, l'istruzione e l'educazione, ne valorizzino il ruolo di futuri cittadini, accompagnandone il processo di responsabilizzazione e protagonismo sociale. La Fondazione seleziona e sostiene quindi progetti che rispondano alle vulnerabilità ma facciano emergere anche risorse e punti di forza, accompagnando percorsi sostenibili di inclusione sociale.

Il progetto "Almeno una stella" rappresenta l'evoluzione e l'ampliamento del precedente "Bussole", in cui circa 250 ragazze e ragazzi di recente immigrazione erano stati sostenuti nel momento cruciale della loro scolarità (la terza media e l'inserimento nella scuola superiore) da giovani studenti, universitari o degli ultimi anni della scuola secondaria di secondo grado, italiani e di origine straniera. Gli studenti, nel ruolo di "tutor" e quasi come "fratelli maggiori" avevano affiancato i docenti e gli insegnanti di italiano lingua seconda nei laboratori didattici e di sostegno all'esame di terza media, sperimentandosi in un ruolo innovativo, con una forte funzione di riferimento positivo per i più piccoli.

Il progetto, realizzato originariamente in quattro città (Milano, Bologna, Torino, Arezzo), si è ampliato in questo biennio a nuovi territori afferenti (la provincia di Trento e il Friuli Venezia Giulia), e ha voluto consolidare il modello di intervento, formalizzando maggiormente la collaborazione con le scuole e le Università tramite accordi, protocolli di intesa e riconoscimento per i tutor di crediti formativi a fronte dell'esperienza realizzata. Anche i numeri sono cresciuti in modo significativo: sono stati raggiunti e seguiti nei due anni 461 alunni, con l'aiuto e il coinvolgimento di oltre 130 tutor.

"Almeno una stella" ha quindi confermato e rinforzato un intervento in grado di agire su un duplice fronte: da un lato la messa a disposizione, a 
scuole ed alunni di recente immigrazione, di risorse e interventi a favore dell'integrazione, in un periodo in cui ormai strutturale ed endemica è la contrazione delle risorse, dei progetti di prevenzione e contro la dispersione scolastica.

Dall'altro la valorizzazione di nuove figure, più vicine per esperienza e vissuto agli alunni, ma sufficientemente più grandi per costituire un modello, un riferimento e, per i tutor stranieri, un'occasione di valorizzazione del percorso di integrazione.

Una figura di prossimità dunque positiva in termini scolastici, linguistici, relazionali, integrativi, che alla fine del progetto precedente necessitava di ulteriori sperimentazioni e messe a punto per poter essere diffusa e utilizzata in diversi contesti.

"Almeno una stella" ha permesso di consolidare tale intervento per altri due anni, confermando gli esiti positivi delle azioni scolastiche e di sostegno relazionale e all'integrazione nel territorio. E ha dimostrato ulteriormente come la figura del tutor sia fortemente generativa, non solo per i destinatari - gli alunni seguiti - ma per gli stessi studenti, che hanno vissuto un percorso di crescita, responsabilizzazione e cittadinanza attiva, utile per il loro futuro professionale ma anche per il loro ruolo di cittadini di domani. E mai come oggi, capace di far emergere e valorizzare gli aspetti più sani e creativi dell'integrazione e del confronto interculturale.

Questo testo rappresenta l'esito di quel percorso, ripartendo dagli esiti di "Bussole" e ripercorrendo la strada compiuta insieme in questi due anni. Il racconto di un'esperienza entusiasmante e coinvolgente, anche a partire dalle parole degli stessi protagonisti, e la messa a disposizione di un vademecum utile a chi volesse provare, anche in altri contesti, a disseminare di "stelle" il cammino verso l'inclusione degli alunni di origine straniera.

Fondazione "Peppino Vismara" Il Presidente Ing. Paolo Morerio 


\title{
Introduzione
}

\author{
di Graziella Favaro
}

"Almeno una stella": nel nome stesso del progetto, sperimentato per due anni in sei diversi territori nazionali, stanno il senso e la finalità dell'azione. Abbiamo preso in prestito l'espressione da Boris Cyrulnik che da anni si occupa della condizione di minori che hanno vissuto una situazione di trauma o di passaggio importante. Egli sostiene che "affinché il bambino o l'adolescente possano intraprendere un cammino di resilienza è necessario che accanto a loro ci sia almeno una stella", e cioè figure, rapporti e relazioni che rappresentino dei veri e propri "tutori di resilienza".

Abbiamo individuato le possibili stelle sul cammino degli adolescenti immigrati nella figura del tutor, un giovane universitario che agisce come un fratello o una sorella maggiori e che può accompagnare per un tratto il cammino di integrazione, sia a scuola che nella città. Il dispositivo di tutoraggio o di mentoring è da tempo largamente diffuso in contesti europei ed extraeuropei e sperimentato con esiti positivi come forma di aiuto e sostegno scolastico, mediazione e rispecchiamento, identificazione positiva e ri-motivazione. In Italia invece non è molto praticato, quantomeno in maniera ordinaria, continuativa e monitorata. Il tutoraggio si rivela fortemente generativo e fecondo e produce cambiamenti importanti e profondi, sia in coloro che sono accompagnati, sia in chi accompagna.

Il progetto "Almeno una stella" è stato sperimentato per due anni a Milano (città capofila), Torino, Bologna, Arezzo, nella provincia di Trento, in alcune città friulane. Abbiamo individuato soprattutto negli adolescenti stranieri di recente immigrazione il gruppo destinatario delle azioni di tutoraggio a causa della criticità dei loro percorsi scolastici e della vulnerabilità della loro condizione di vita subito dopo l'arrivo. Per le ragazze e i ragazzi che migrano, il viaggio di migrazione rappresenta infatti un vero e proprio snodo biografico che segna profondamente il prima e il dopo. Essi si trovano in tempi brevissimi e all'improvviso, spesso senza averlo scelto, a vivere separazioni e rotture importanti e molteplici: dal luogo di origine 
e dalle figure parentali che li avevano accuditi fino a quel momento, dagli amici rimasti in patria, dalla scuola che li ha visti crescere e apprendere. Quando arrivano in Italia, devono affrontare sfide importanti (imparare la lingua, ri-orientarsi, stabilire nuovi legami di amicizia...) spesso in solitudine e senza poter contare su aiuti efficaci e su figure di accompagnamento dedicate. Le criticità scolastiche che disseminano il percorso di integrazione delle ragazze e dei ragazzi di recente immigrazione, se non precocemente affrontate, tendono inoltre a moltiplicarsi, anziché ridursi.

Il progetto "Almeno una stella" ha cercato di organizzare una rete di accoglienza e di accompagnamento per gli adolescenti neoarrivati, prevedendo azioni dedicate e di qualità per l'apprendimento della lingua e l'aiuto allo studio, per sostenere il momento dell'orientamento e la fase di passaggio dalla scuola secondaria di primo a quella di secondo grado, per far conoscere e abitare la città e i suoi luoghi di aggregazione.

Lo abbiamo fatto, affiancando agli insegnanti specialisti i giovani tutor. Chi sono i tutor coinvolti nel progetto? Si è trattato di 131 giovani, per lo più universitari, metà dei quali di origine straniera e quindi con un vissuto di migrazione comune agli adolescenti accompagnati. Sono quindi figure di prossimità per vari aspetti: la vicinanza anagrafica e di età, le esperienze di vita molto simili, le lingue e i linguaggi condivisi. Sono quindi vicini pur senza essere "pari": né compagni di classe né adulti, ma collocati in quello spazio della giusta distanza che li rende prossimi come fratelli e sorelle maggiori. L'esperienza di tutoraggio è stata estremamente positiva per gli adolescenti accompagnati, ma lo è stata in ugual misura per i giovani tutor che hanno rivestito per uno o due anni questo ruolo. È stata per loro l'occasione per prendersi cura di qualcuno che attraversava una situazione transitoria di vulnerabilità, per vivere concretamente un ruolo di responsabilità nei confronti dei più piccoli, per sperimentare davvero un'occasione di cittadinanza attiva. I diari di bordo che tutti i tutor hanno compilato per raccontare la loro esperienza ne danno chiara testimonianza. "È molto di più quello che ho ricevuto da questa esperienza, rispetto a quello che ho dato": questa affermazione si ritrova spesso nelle testimonianze dei giovani universitari.

Il testo racconta il progetto e le sue azioni. Lo fa partendo dalla presentazione del contesto nel quale i tutor si sono trovati ad agire e cioè la scuola multiculturale, spesso impreparata di fronte alle "nuove adolescenze" e impoverita quanto a risorse e strumenti (cap. 1). Il secondo capitolo entra in maniera dettagliata nella descrizione delle azioni condotte nei territori coinvolti e dà conto delle diverse declinazioni progettuali messe in atto nelle città e nelle scuole che hanno partecipato. I capitoli 3 e 4 descrivono gli adolescenti immigrati prestando attenzione ad aspetti salienti del loro viaggio e della loro storia, alle fatiche e alle conquiste (cap. 3) e commen- 
tando i dati quantitativi riferiti ai loro cammini scolastici e gli esiti seguiti alle azioni.

Gli ultimi capitoli del testo sono dedicati alla figure dei tutor e al dispositivo del tutoraggio, alle fasi e modalità degli interventi (cap. 5). Vengono approfonditi i modi diversi di essere tutor: ora attento alla relazione, ora guida alla scoperta della città; a volte figura impegnata a costruire resilienza e a sostenere l'autostima, altre volte a scoprire come rabdomante talenti e capacità nascosti degli adolescenti che vengono da lontano (cap. 6).

L'ultimo capitolo infine ha un carattere operativo e di proposta concreta: propone infatti un esempio di vademecum in dieci punti per realizzare un progetto di tutoraggio da realizzare dentro la scuole e nella città.

Il racconto dell'esperienza fortemente positiva di "Almeno una stella" per tutti i soggetti coinvolti e la proposta di un vademecum operativo vogliono essere altrettanti inviti a diffondere e a sperimentare questo dispositivo, coinvolgendo sempre di più i giovani studenti universitari a vivere un'esperienza che segnerà in maniera positiva il loro diventare adulti. 



\title{
1. Uno sguardo alla scuola multiculturale e ai suoi protagonisti
}

\author{
di Graziella Favaro
}

Dialogo tra Emma e suo nonno.

N. Com'è la tua classe?

E. Normale.

N. Ci sono tanti stranieri?

E. No, nella mia classe ci sono solo bambini!

Uno sguardo diacronico alla scuola multiculturale ci consente di cogliere le diverse fasi attraversate in questi anni, il "clima" che si respira dentro le aule e di fotografare i percorsi scolastici distinguendo tra gli alunni di "prima e di seconda" generazione. Vecchi e nuovi temi, attenzioni consolidate e criticità emergenti si intrecciano e si impongono oggi come prioritari. Mentre il numero dei bambini e dei ragazzi dell'immigrazione si assesta - e, anzi, tende a diminuire e si diffonde una "normale eterogeneità" molte domande restano ancora senza risposta.

Nelle note che seguono, proviamo a raccontare la scuola multiculturale evidenziandone le luci e le ombre; cogliamo le rappresentazioni diverse che fanno da sfondo alla normativa e presentiamo i percorsi scolastici dei bambini e dei ragazzi "nuovi italiani" che spesso hanno ancora il segno di un'integrazione rallentata.

\section{Due priorità, un "modello asistematico"}

L'immigrazione ha profondamente cambiato la scuola.

Uno sguardo alle classi dei più piccoli e dei più grandi ci restituisce l'immagine di una scuola fortemente segnata dall'eterogeneità e che continua a modificarsi, anno dopo anno, in maniera veloce, rispetto alle storie, ai viaggi, ai volti e alle biografie di coloro che la abitano. Ci conferma inoltre che la presenza dei figli dell'immigrazione negli spazi educativi e nei luoghi di aggregazione e di incontro è uno dei tratti più rilevanti che segnano la fisionomia e il paesaggio sociale delle nostre città e comunità locali.

I luoghi della formazione e le scuole sono stati, e continuano a essere, i primi e principali contesti pubblici a vivere il confronto con le differenze di origine, lingua, bagagli autobiografici, riferimenti culturali. In questi 
anni, i servizi educativi e le istituzioni educative hanno accolto un numero crescente di bambini e ragazzi stranieri, venuti da lontano o nati in Italia. Hanno imparato, in molti casi, ad ascoltare le aspettative e i messaggi, espliciti e impliciti, delle famiglie straniere e italiane, a tener conto dei loro timori e delle attese, degli impacci e delle assenze. Hanno sperimentato qua e là azioni innovative, volte a dare risposta a bisogni linguistici e didattici specifici, a conoscere almeno un po' la storia che fa da sfondo ai viaggi e alla migrazione dei minori, a leggere nel loro silenzio il lavorio sorprendente dell'acquisizione della nuova lingua e, talora, anche il dolore della perdita e della nostalgia. A volte hanno fatto un po' di resistenza di fronte ai cambiamenti inevitabili che le trasformazioni del paesaggio scolastico e sociale necessariamente comportano. In tutti i casi, le consapevolezze, le acquisizioni, le modalità didattiche e organizzative innovative e che si sono rivelate maggiormente efficaci non sono ancora diventate sistema e prassi condivisa.

Due attenzioni pedagogiche e didattiche sono alla base di una scuola multiculturale e multilingue efficace e di qualità. Da un lato, vi è l'attenzione mirata all'accoglienza degli alunni stranieri che si inseriscono per la prima volta nella scuola italiana; dall'altro lato, vi è la gestione educativa della classe normalmente eterogenea attraverso un approccio interculturale e plurilingue. Si tratta quindi di procedere contemporaneamente lungo due direzioni e di tenere insieme i due percorsi, attivando un doppio sguardo: quello "dedicato", che ha carattere transitorio e specifico, e quello ordinario e per tutti.

Chi sono gli alunni neoarrivati? Si tratta ad ogni anno scolastico di circa 30.000 alunni (4,9\% sul totale degli stranieri), metà dei quali entra nella scuola primaria. Essi provengono direttamente dal Paese d'origine per ricongiungimento famigliare o a causa di esodo e fuga da guerre e condizioni di vita drammatiche. In altri casi, sono nati qui ma non hanno mai frequentato la scuola dell'infanzia e sono quindi "nuovi" alla scuola. A proposito di alunni neoinseriti, uno studio comparato promosso dall'Unione Europea (Study on educational support for newly arrived migrant children) ha analizzato la situazione di 15 Paesi dell'Unione, fra i quali vi è l'Italia. Il modello italiano viene definito, insieme a quello greco, "non sistematico": "Il modello non sistematico funziona in maniera random. I Paesi non hanno una politica chiaramente articolata a livello nazionale e il supporto previsto è molto frammentato. Il sistema scolastico italiano è fortemente centralizzato, ma le scuole sono lasciate a se stesse nella scelta dei metodi d'insegnamento e nel far fronte alla diversità della popolazione scolastica locale. Le linee guida nazionali non prevedono un supporto linguistico e scolastico strutturato e ben definito per gli alunni migranti". 
Il documento offre un quadro esaustivo e molto interessante degli approcci e delle misure realizzate nei diversi contesti e presenta anche alcune pratiche e progetti che hanno avuto esiti efficaci e positivi.

In particolare, sono quattro i dispositivi che lo studio ritiene centrali per inaugurare un percorso di buona integrazione e sono:

- il supporto linguistico mirato in L2 nella fase iniziale e per tutto il tempo che è necessario;

- l'aiuto allo studio realizzato in maniera duratura e continuativa;

- il coinvolgimento delle famiglie degli alunni neoinseriti e la collaborazione con il territorio per una buona integrazione anche nell'extra scuola;

- l'approccio interculturale.

\section{Bisogni educativi specifici o speciali?}

Più di vent'anni di parole e pratiche d'integrazione dovrebbero costituire ormai una robusta riserva di esperienze e di risposte educative utili a comporre un "modello" d'integrazione condiviso e riconoscibile, efficace nel ridurre le disparità che si registrano tuttora da una scuola all'altra, da una città all'altra. Ma questo processo non è ancora compiuto e si notano tuttora divari importanti, scelte differenti, risorse e dispositivi non uniformi da un contesto educativo all'altro, dando luogo appunto a un "modello non sistematico".

La normativa stessa, che descrive e regola il tema - e che di fatto contribuisce a costruire le cornici pedagogiche e di senso del percorso d'integrazione - propone rappresentazioni a volte tra loro discordanti. In essa si sottolinea infatti, ora la "normale eterogeneità" delle classi e dei gruppi di apprendimento, ora il carattere "speciale" delle domande educative provenienti dagli alunni con cittadinanza non italiana.

Ripercorriamo i documenti più recenti - dal 2010 a oggi - per cogliere le parole chiave, gli scenari evocati, le risposte previste o sollecitate. Nel gennaio 2010, una circolare sulle iscrizioni suscitò vivaci prese di posizione e alcune polemiche poiché introduceva il "tetto" del 30\% per gli alunni stranieri nelle classi prime. Seguirono deroghe, precisazioni, chiarimenti. Ma la rappresentazione che la circolare ha contribuito a veicolare e a diffondere nella scuola è stata quella degli alunni non (ancora) italiani, anche se nati in Italia, come un'emergenza da contenere, un flusso da arginare. Due altri importanti e successivi documenti propongono la situazione di multiculturalità di fatto in maniera ancora diversa. Il primo - le indicazioni nazionali per il curricolo della scuola di base del 2012 - prende atto che "una molteplicità di lingue e culture è entrata nella scuola" e sollecita $\mathrm{i}$ docenti a "progettare e realizzare percorsi didattici specifici" per lo svilup- 
po dell'italiano per comunicare e per lo studio, con l'obiettivo della piena integrazione (senza tuttavia indicare modalità e risorse sul come fare). Il secondo documento - la circolare applicativa sui BES, i bisogni educativi speciali, del 6 marzo 2013 - tratta degli alunni più "fragili" e mette insieme coloro che hanno una disabilità, un DSA (disturbo specifico dell'apprendimento), o disturbi evolutivi specifici, con chi si colloca "nell'area dello svantaggio socioeconomico, linguistico e culturale". Per rispondere a tali bisogni educativi speciali si suggeriscono misure a carattere compensativo e dispensativo.

Le Linee guida sull'accoglienza e l'integrazione degli alunni stranieri (MIUR, 2014) ripropongono uno sguardo specifico, e non speciale, e sono più attente, rispetto al passato, ai temi emergenti dell'orientamento e della prosecuzione degli studi, dell'insegnamento dell'italiano L2 e della valorizzazione della diversità linguistica.

Dalla lettura dei documenti ufficiali, quale rappresentazione si può dunque cogliere della scuola italiana e dei bambini e dei ragazzi stranieri che la abitano? E quali scenari, scelte pedagogiche, mosse didattiche essi propongono e sollecitano?

Le categorie presenti nelle descrizioni dei contesti educativi e scolastici multiculturali e delle attenzioni da promuovere sono, come abbiamo visto, alquanto differenti. Da un lato, si presenta la situazione di mescolanza ed eterogeneità come normale (anche se certamente essa necessita di essere gestita e supportata da attenzioni didattiche e dispositivi mirati). Dall'altro lato, ci si richiama al carattere di "specialità" delle domande e dei bisogni educativi, riconducendoli alla categoria della mancanza e del disagio. Si oscilla dunque tra l'etnicizzazione e l'irrigidimento dei cammini di apprendimento degli alunni non italiani (il "tetto" del 30\%; la contiguità tra l'area dello "svantaggio socioeconomico, linguistico e culturale" e le situazioni della disabilità) e la rappresentazione, invece, di una "normale" eterogeneità, pur tuttavia senza proporre misure e risorse specifiche e a carattere transitorio.

Le esperienze condotte in questi anni, e il confronto con altri contesti educativi nazionali che sono da tempo multiculturali e plurilingui, suggeriscono che il tema dell'inserimento scolastico degli alunni che continuiamo a definire "stranieri" ha carattere specifico - e non speciale - e richiede attenzioni e piani didattici e linguistici "ordinari", efficaci e mirati.

Non invisibilità dunque, ma neppure forme di etichettamento che rischiano di irrigidire le condizioni che si presentano fluide e in movimento e di rendere permanenti i bisogni transitori con il rischio di creare distanze e forme di microsegregazioni. 


\section{Un Vademecum per l'integrazione}

Nel settembre 2015, un nuovo documento è stato diffuso alle scuole dal MIUR. Si tratta del Vademecum per l'integrazione "Diversi da chi?", redatto da chi scrive per conto dell'Osservatorio nazionale per l'integrazione e l'intercultura. Dopo una breve premessa, il documento individua e propone dieci obiettivi e priorità per una scuola inclusiva, indicando per ciascuna azioni possibili e modalità operative per l'attuazione.

1. Ribadire il diritto all'inserimento immediato degli alunni neoarrivati.

2. Rendere consapevoli dell'importanza della scuola dell'infanzia.

3. Contrastare il ritardo scolastico.

4. Accompagnare i passaggi; adattare il programma e la valutazione.

5. Organizzare un orientamento efficace alla prosecuzione degli studi. Investire sul protagonismo degli studenti.

6. Sostenere l'apprendimento dell'italiano L2, lingua di scolarità.

7. Valorizzare la diversità linguistica.

8. Prevenire la segregazione scolastica.

9. Coinvolgere le famiglie nel progetto educativo per i loro figli.

10. Promuovere l'educazione interculturale nelle scuole.

\section{Tra ostacoli e attese}

Se diamo uno sguardo ai percorsi di inserimento scolastico dei minori che hanno una storia, personale o famigliare, di migrazione, così come essi vengono descritti e raccontati nei dati nazionali e nelle ricerche locali, vediamo che sono soprattutto cinque le criticità disseminate lungo un cammino segnato da fatiche, conquiste e rallentamenti.

Proviamo a descriverli in maniera sintetica. Lo facciamo, proponendo anche $\mathrm{i}$ dati e le storie raccolti in un gruppo/campione significativo, costituito dalle ragazze e dai ragazzi coinvolti nel progetto di tutoraggio "Almeno una stella".

\section{- Adolescenti sulla soglia: le difficoltà di ingresso nella scuola}

Cesar, arrivato dal Perù per ricongiungersi alla madre nel gennaio di due anni fa, ha potuto entrare a scuola solo a settembre, dopo nove mesi di attesa e di vuoto, perché in tutte le scuole di zona alle quali ha bussato "non c'era posto". Xia Feng, quattordicenne neoarrivato dalla Cina, è stato "rimbalzato" per mesi tra la scuola secondaria di primo grado vicino a casa e le scuole superiori della zona. Per la scuola media, era infatti considerato troppo grande; per le scuole secondarie di secondo grado, era considerato inadatto a causa della sua non conoscenza dell'italiano. Come Cesar e Xia Feng, una parte dei ragazzi stranieri si "disperde" e non viene 
inserita nella scuola subito dopo l'arrivo, oppure trascorre un lasso di tempo considerevole fra il momento del ricongiungimento famigliare e quello dell'ingresso nella classe.

Quali sono i soggetti più a rischio e i fattori che sono alla base di queste forme di "descolarizzazione" di fatto, durature o transitorie che esse siano? In alcuni casi, possono essere le famiglie, sprovviste delle corrette informazioni, ad avere comportamenti incerti nei confronti dell'inserimento scolastico e a non promuovere l'ingresso immediato del figlio (o della figlia) nella scuola italiana. In altri casi, sono invece le scuole a non accogliere la domanda di inserimento - o a non accoglierla subito - per varie ragioni: il momento dell'anno in cui i minori si presentano, la situazione sempre più diffusa di saturazione delle classi, la mancanza di risorse specifiche. Nonostante la normativa preveda "l'inserimento dell'alunno in qualunque momento dell'anno arrivi", nella realtà vi sono dunque ragazze e ragazzi che cercano a lungo un posto a scuola prima di approdare a destinazione. Sono soprattutto gli adolescenti e coloro che arrivano in Italia in corso d'anno scolastico (e i maschi più delle femmine) a trovarsi per un po' "fuori dalla porta", con il rischio di perdere del tempo prezioso, allentare la motivazione ad apprendere, ridurre la possibilità di contatto e scambio con i coetanei italiani.

\section{- Indietro di uno o più anni: il ritardo scolastico}

Fra i 458 adolescenti coinvolti nel progetto "Almeno una stella" per i quali il dato risulta disponibile, solo il $35 \%$ risulta essere nella condizione di "parità" tra età e classe frequentata, mentre il $65 \%$ si trova in situazione di ritardo: di un anno nel 38,8\% dei casi; di due, tre e fino a sei (!) anni nel $26,2 \%$. Una parte consistente degli alunni stranieri continua infatti a essere inserita al momento dell'arrivo in Italia in un classe non corrispondente all'età anagrafica. La situazione di ritardo penalizza in maniera particolare gli alunni inseriti nella scuola secondaria di primo e secondo grado e pregiudica spesso la possibilità di prosecuzione nella carriera scolastica. I dati raccolti dal MIUR indicano una percentuale ancora preoccupante di alunni in situazione di ritardo scolastico, anche se essa tende a diminuire in seguito all'inserimento consistente delle "seconde generazioni" che registrano una maggiore corrispondenza tra età e classe.

Sono oggi in questa condizione:

- il $14,7 \%$ degli alunni stranieri nella scuola primaria;

- il $41,5 \%$ nella scuola secondaria di primo grado;

- il $65,1 \%$ nella scuola secondaria di secondo grado.

Le variabili che sono alla base della situazione di ritardo sono sostanzialmente due. La prima - ancora oggi la più rilevante - ha a che fare con 
la scelta iniziale della classe in cui inserire l'alunno straniero. Anche in questo caso, nonostante la normativa preveda che il criterio privilegiato per la determinazione della classe di inserimento sia quello dell'età anagrafica, si registra di fatto un comportamento di penalizzazione nei confronti degli alunni con cittadinanza non italiana, i quali vengono in gran parte retrocessi, come abbiamo visto, di uno, due e a volte anche tre anni.

\section{- Bocciati in prima: l'insuccesso scolastico}

La seconda variabile che provoca il ritardo scolastico riguarda invece gli esiti scolastici a fine anno e il tasso di promozione/bocciatura che si registra fra gli stranieri. Il divario medio tra allievi italiani e stranieri è rilevante fin dal primo ciclo di scuola e si presenta inoltre particolarmente pesante nelle prime classi di ogni ordine di scuola. Nella primaria, ad esempio, la differenza tra i bambini italiani e stranieri che vengono bocciati alla fine del primo anno è di circa due punti; nella scuola secondaria di primo grado, alla fine della prima media si registra un esito negativo per il 3\% degli italiani e ben il 10,2 degli alunni stranieri. Al termine della prima classe delle superiori, viene "fermato" l' $8,6 \%$ degli studenti italiani e il $12,2 \%$ degli stranieri. Ma in questo anno di scolarità si registrano anche numerosi ritiri e abbandoni da parte degli allievi non italiani.

Si può costruire dunque nel tempo una sorta di vulnerabilità persistente e di circolo vizioso che si origina dalla condizione di migrazione e dall'essere straniero: un inserimento penalizzante in ingresso, che non rispetta la coerenza tra età e classe frequentata; la probabilità maggiore di avere un esito negativo soprattutto alla fine del primo anno di ogni tipo di scuola; una marginalità sociale che diventa anche solitudine relazionale nel tempo extrascolastico; la prossimità quotidiana nei confronti di altri alunni con scarse performance che crea un effetto moltiplicatore della vulnerabilità e rischia di produrre demotivazione e abbandoni.

\section{- Scelte nella prosecuzione degli studi}

Una parte consistente degli alunni stranieri ha difficoltà a proseguire gli studi dopo la secondaria di primo grado: ricerche a livello locale mostrano tassi elevati di abbandono dopo il primo anno delle superiori, numerosi "scivolamenti" verso il basso e un addensamento delle presenze nei percorsi di formazione brevi e meno esigenti. I dati del MIUR lo confermano: il $37,9 \%$ dei ragazzi stranieri nati all'estero si orienta verso gli istituti professionali, mentre si indirizza verso questo percorso di istruzione il $19 \%$ circa degli alunni italiani. Viceversa, frequentano una qualunque classe liceale il $28 \%$ circa degli allievi non italiani e quasi il $48 \%$ degli studenti autoctoni.

$\mathrm{Si}$ osserva inoltre che si orientano verso le scuole a carattere professionalizzante anche gli alunni stranieri che ottengono buoni risultati all'esame di terza media. Vi è dunque il rischio che le ragazze e i ragazzi stranieri 
facciano (o siano orientati verso) scelte scolastiche e più brevi e meno esigenti con la conseguente dispersione di talenti e possibilità che andrebbero invece sostenuti e valorizzati.

\section{L'italiano di scolarità: la tigre sul cammino}

Alla base delle criticità descritte sopra viene spesso indicata la non competenza o una padronanza ridotta nella lingua italiana, non tanto per gli usi comunicativi, quanto per le abilità di studio (Italstudio). Ed è questo il quinto fattore e ostacolo che gli alunni stranieri incontrano sul loro cammino: una vera e propria "tigre sul cammino", come i cinesi definiscono le difficoltà più ardue. Se infatti l'acquisizione dell'italiano per comunicare avviene per gran parte degli alunni stranieri in tempi relativamente brevi - grazie anche ai contatti numerosi e densi con i pari a scuola e nel tempo libero - l'apprendimento della lingua veicolare implica tempi lunghi e può avvenire solo all'interno della scuola. Esso richiede modalità didattiche protratte di facilitazione e semplificazione, materiali didattici efficaci, attenzioni da parte di tutti gli insegnanti, ognuno dei quali, oltre ai contenuti disciplinari, trasmette anche la microlingua delle discipline. Dispositivi, consapevolezze e risorse di cui spesso le scuole non hanno la disponibilità.

A proposito di competenza linguistica degli alunni stranieri, sono disponibili i risultati delle prove INVALSI, divisi per origine degli alunni e per regione (www.invalsi.it/snv).

Nella Tab. 1 possiamo leggere le differenze fra i punteggi in italiano degli alunni italiani e non italiani e, fra questi, distinguere tra le performance degli allievi di prima e di seconda generazione.

Tab. 1 - Differenza percentuale fra alunni stranieri e alunni italiani nelle risposte al test di italiano, SNV Invalsi

\begin{tabular}{|l|l|c|c|c|c|c|}
\hline \multicolumn{2}{|c|}{ Gruppo } & $\begin{array}{c}\mathbf{2}^{\circ} \\
\text { primaria }\end{array}$ & $\begin{array}{c}\mathbf{5}^{\circ} \\
\text { primaria }\end{array}$ & $\begin{array}{c}\mathbf{1}^{\circ} \\
\text { secondaria }\end{array}$ & $\begin{array}{c}\mathbf{2}^{\circ} \\
\text { secondaria }\end{array}$ & $\begin{array}{c}\mathbf{3}^{\circ} \\
\text { secondaria }\end{array}$ \\
\hline \multirow{2}{*}{$\mathbf{2 0 1 2 / 1 3}$} & \multicolumn{2}{|c|}{ I grado } & \multicolumn{3}{|c|}{ II grado } \\
\cline { 2 - 7 } & I generazione & -27 & -14 & -34 & -22 & -14 \\
\cline { 2 - 7 } & II generazione & -15 & -11 & -16 & -6 & -13 \\
\hline \multirow{2}{*}{$\mathbf{2 0 1 0 / 1 1}$} & I generazione & $-18,8$ & $-13,7$ & $-21,1$ & $-9,5$ & $-15,3$ \\
\cline { 2 - 7 } & II generazione & $-14,1$ & $-7,3$ & $-11,1$ & $-5,7$ & $-7,7$ \\
\hline \multirow{2}{*}{$\mathbf{0 0 9 / 1 0}$} & I generazione & $-27,4$ & $-21,7$ & $-20,2$ & n.d. & n.d. \\
\cline { 2 - 7 } & II generazione & $-21,5$ & $-11,7$ & $-9,7$ & n.d. & n.d. \\
\hline
\end{tabular}

Fonte: elaborazioni su dati Invalsi (2009; 2010; 2013) 
Dall'osservazione dei dati, possiamo trarre alcune considerazioni.

- Le differenze del punteggio ottenuto dagli alunni stranieri rimangono nel tempo significative, rispetto a quello degli autoctoni.

- I risultati ottenuti dagli alunni appartenenti alla cosiddetta "seconda generazione", costituita dai nati in Italia, sono migliori rispetto a quelli degli alunni stranieri nati all'estero e inseriti nella scuola italiana ad un certo momento della loro vita. Le differenze di punteggio ottenuto dagli alunni nati in Italia, rispetto ai compagni italiani, sono, ad esempio, di 15 punti in seconda primaria (ma salgono a 27 punti per la "prima generazione"); di circa 11 punti in quinta (e di 14 punti per i bambini nati all'estero); di 16 punti in prima media (e ben 34 per i nati all'estero); di 6 punti in terza media (a fronte di 22 punti per i ragazzi ricongiunti).

- Si notano dei miglioramenti nel corso degli anni all'interno di uno stesso grado scolastico: vi è infatti una riduzione del divario fra la seconda e la quinta primaria e fra la prima media e la terza media.

- I risultati migliorano con il progredire dell'istruzione all'interno di ogni tipo di scuola, come abbiamo visto, ma tendono a peggiorare nel passaggio da un ordine di scuola all'altro. Se osserviamo, ad esempio, i dati 2012/13 relativi alla seconda generazione, vediamo che vi è un aumento del gap fra la quinta primaria e la prima media e fra la terza media e la seconda superiore. II dato evidenzia ancora una volta la difficoltà che esiste nella scuola italiana nei passaggi da un grado di scuola all'altro; fra un ciclo di studi e quello successivo.

Le considerazioni che possiamo trarre dai dati INVALSI ci invitano a riflettere soprattutto su alcuni temi: la competenza in italiano dei più piccoli nati in Italia (che ottengono risultati inferiori agli italiani in seconda elementare); la necessità di potenziare l'insegnamento dell'italiano per lo studio; i passaggi da un tipo di scuola ad un altro, momento in cui si registrano criticità, esiti scolastici negativi e abbandoni.

\section{I fattori collegati al rischio della dispersione scolastica}

Un recente studio condotto a Milano sulla dispersione, sulla base dei dati dell'anagrafe scolastica cittadina, ha evidenziato che il rischio di abbandono della scuola tra gli alunni stranieri nati all'estero è dieci volte superiore a quello degli italiani nativi e quattro volte superiore a quello degli studenti non italiani di seconda generazione (Bugli et al., 2015). Quali sono gli eventi che sono alla base della dispersione o che ne acuiscono il rischio? Sono soprattutto tre fattori:

- la ripetenza della classe in corso;

- il ritardo scolastico e il conseguente divario tra età e classe frequentata;

- il non superamento dell'anno. 
Il dato del ritardo scolastico pesa dunque in maniera determinante sulla dispersione ed è inoltre un predittore di altri eventi critici quali: ripetenze, perdita di motivazione, scelte al ribasso nella prosecuzione degli studi, frequenza saltuaria o abbandono della scuola.

Quali sono le nazionalità per le quali gli eventi connessi al rischio di dispersione pesano in misura maggiore? La situazione milanese colloca al primo posto gli alunni romeni. A fronte di una tasso medio di rischio di interruzione scolastica non formalizzata pari allo $0,38 \%$, i ragazzi romeni registrano il 3,87\%; seguono gli egiziani (1,75\%); i singalesi (1,32\%), i cinesi $(1,29 \%)$.

Naturalmente, le storie di migrazione, la situazione famigliare e il capitale culturale dei genitori pesano anch'essi in maniera determinante sul rischio di dispersione scolastica dei figli. La ricerca milanese mette tuttavia in luce che anche quando è disponibile un capitale culturale dei genitori relativamente alto, con un titolo equivalente al diploma universitario o alla laurea, le differenze di successo scolastico continuano a permanere, a scapito degli alunni con cittadinanza non italiana. In altre parole, il fatto di essere straniero, pur se figlio di laureati, sembra pesare di per sé come condizione di svantaggio alla partenza.

\section{I più piccoli, i più grandi}

Come abbiamo visto, i percorsi scolastici dei bambini e dei ragazzi stranieri presentano delle criticità che permangono nel tempo. Ma una scuola impoverita e priva di risorse rischia di non poter dare risposte efficaci, continuative e mirate, ai bisogni di accoglienza, di apprendimento linguistico - per gli usi comunicativi e per quelli scolastici - di orientamento dei "nuovi" alunni. Alla scarsità dei dispositivi e delle attenzioni per una buona integrazione che connota la scuola, si deve aggiungere inoltre l'impossibilità di gran parte delle famiglie immigrate a sostenere i loro figli nello studio, a controllarne il percorso, a supportare le scelte per il futuro.

I bambini e i ragazzi stranieri sono dunque spesso soli davanti ai compiti di studio e nel tempo extrascolastico e devono contare soprattutto sulle loro risorse personali: sui saperi e le capacità consolidati nella scuola precedente, sull'autodisciplina e il senso del dovere, sulle attitudini personali e la voglia di riuscire. E i risultati positivi di una parte degli alunni non italiani, registrati anche alla recente sessione degli esami di terza media e di maturità, lo confermano.

Ma quali priorità dovrebbero essere poste al centro di una scuola che sia davvero in grado di includere tutti? Ne indichiamo cinque. 
- un'attenzione ai più piccoli poiché una buona integrazione si inaugura nella prima infanzia. Ciò significa la promozione dell'inserimento massiccio e diffuso dei bimbi stranieri nella scuola dell'infanzia, cosa che oggi non avviene, dal momento che circa un quarto dei piccoli non italiani non la frequenta, contrariamente a quanto avviene per i bambini autoctoni, i quali vi sono inseriti per la quasi totalità. II tempo compreso fra i tre e i sei anni è infatti cruciale ai fini dell'acquisizione della seconda lingua, della socializzazione fra pari, del successivo ingresso nella lingua scritta;

- un piano nazionale di accoglienza e di insegnamento dell'italiano come seconda lingua - per comunicare e per studiare - rivolto, in particolare, agli adolescenti che arrivano per ricongiungimento famigliare, i quali attraversano le difficoltà scolastiche più evidenti e si trovano ad apprendere il nuovo codice in un'età definita "critica";

- un'attenzione particolare ai momenti di passaggio, dal momento che le criticità più rilevanti degli alunni stranieri si collocano, come abbiamo visto, al primo anno di ogni tipo di scuola;

- una rete di aiuto allo studio, nel tempo scolastico ed extrascolastico, che possa accompagnare gli alunni stranieri fuori dalla scuola con forme mirate di tutoraggio, sostegno all'apprendimento, rimotivazione, gestite da voIontari competenti, associazioni educative professionali. Una rete che può coinvolgere anche studenti universitari, italiani e stranieri, i quali potrebbero svolgere efficacemente la funzione di tutor o mentore e inoltre vivere concretamente un ruolo di cittadinanza attiva, come dimostra il progetto "Almeno una stella";

- un'attenzione mirata all'orientamento scolastico e alle modalità di prosecuzione degli studi, perché questo momento di scelta può segnare l'inizio di un processo di marginalizzazione sociale e lavorativa, oltre che scolastica o, viceversa, rappresentare un'opportunità di mobilità e di promozione $e$ inaugurare un percorso di inclusione positiva e di riconoscimento di talenti e capacità.

\section{Riferimenti bibliografici}

Bugli V., Conte M., Laffi S. (2015), La dispersione a Milano nei dati dell'Anagrafe scolastica. Anno scolastico 2013-14, in www.comune.milano.it.

Checchi D. (a cura di) (2014), Lost. Dispersione scolastica: il costo per la collettività e il ruolo di scuole e Terzo settore, in www.weworld.it; www. trentin.cgil.it; www.fga.it.

Colombo E. (a cura di) (2010), Figli di migranti in Italia. Identificazioni, relazioni, pratiche, Utet, Torino.

Dalla Zuanna G., Farina P., Strozza S. (2009), Nuovi italiani. I giovani immigrati cambieranno il nostro Paese?, Il Mulino, Bologna.

Favaro G. (2014 n.e.), A scuola nessuno è straniero. Insegnare e apprendere nella classe multiculturale, Giunti, Firenze.

Favaro G. (2013), Bussole: i più grandi accompagnano i più piccoli. Un progetto di tutoring scolastico per gli adolescenti stranieri, Centro COME, Milano (www.centrocome.it). 
Favaro G., Papa N. (2009), Le ragazze e i ragazzi stranieri nella scuola superiore, FrancoAngeli, Milano.

Favaro G. (2014), Un'integrazione rallentata: bambini e ragazzi “stranieri” nella scuola di tutti, in Fondazione "Moressa", L'economia dell'immigrazione n. 5.

Granata A. (2011), Sono qui da una vita. Dialogo aperto con le seconde generazioni, Carocci, Roma.

MIUR-ISMU (2011), Alunni con cittadinanza non italiana. Verso l'adolescenza. Rapporto nazionale anno scolastico 2010-2011, www.istruzione.it.

MIUR-ISMU (2015), Alunni con cittadinanza non italiana. Tra difficoltà e successi. Rapporto nazionale a.s. 2013/2014, www.istruzione.it.

Ongini V. (2011), Noi domani. Un viaggio nella scuola multiculturale, Laterza, Bari.

Queirolo Palmas L. (2006), Prove di seconde generazioni. Giovani di origine immigrata tra scuole e spazi urbani, FrancoAngeli, Milano.

Ravecca A. (2009), Studiare nonostante. Capitale sociale e successo scolastico degli studenti di origine immigrata nella scuola superiore, FrancoAngeli, Milano.

Unione Europea (2013), Study on educational support for newly migrant children. Final report: www.ec.europe.eu/education. 


\title{
2. Il progetto e le azioni nelle città
}

\author{
di Michela Casorati ${ }^{\star}$ e Monica Napoli
}

\section{A partire dalle "Bussole"... Alle origini del progetto}

Per gli alunni di origine straniera, in particolar modo neoarrivati, l'inserimento nella scuola e nel territorio rappresenta una sfida aggiuntiva alla già complessa transizione dall'infanzia all'adolescenza, come abbiamo visto nel primo capitolo. Gli ormai innumerevoli studi sia a livello nazionale che europeo testimoniamo la fragilità dei cammini formativi (MIUR e Fondazione Ismu, 2014; OECD, 2012, 2013, 2014; Rete europea Eurydice); e le criticità dell'inclusione in ambito socio-educativo (Dalla Zuanna, Farina, Strozza, 2009), che si accentuano con il diminuire degli anni di presenza nel paese di accoglienza.

In tale contesto di precarietà, e per favorire i percorsi di integrazione e riuscita scolastica, abbiamo provato a sperimentare il valore di nuove figure di riferimento, quali i giovani tutor italiani e di origine straniera da affiancare agli alunni e studenti stranieri, in particolare nel passaggio critico di uscita dalla scuola media e ingresso nella secondaria di secondo grado.

Il progetto "Almeno una stella", ideato e coordinato dal Centro COME della cooperativa Farsi Prossimo e finanziato dalla Fondazione "Peppino Vismara", rappresenta la prosecuzione del progetto "Bussole", già sostenuto dalla Fondazione e realizzato in quattro città italiane (Milano, Bologna, Torino e Arezzo) nel 2011 e 2012 (Favaro, 2013). In quella prima esperienza, avevamo provato a disseminare lungo il percorso dell'integrazione di ragazzi stranieri presenti nelle quattro città alcune "bussole" che ne facilitassero l'orientamento, alcuni "tutori di resilienza" che potessero affiancare ed aiutare gli studenti sia nel corso di laboratori per l'acquisizione dell'italiano in tempo scolastico ed extrascolastico (ad esempio per la preparazione dell'esame di terza media o nell'inserimento in prima superiore),

* Centro Come - Cooperativa Farsi Prossimo, Milano. 
sia per quanto attiene le dinamiche più relative alla dimensione relazionale ed affettiva, sostenendone la valorizzazione, il riconoscimento, lo scambio, con i pari e tra culture differenti.

I tutor individuati erano giovani studenti, della scuola secondaria di secondo grado o universitari, italiani o, per la maggior parte, essi stessi di origine straniera, e che avevano quindi sperimentato, prima dei loro "tutorati" le difficoltà dell'inserimento e la necessità di essere guidati ed accompagnati da "una stella sul loro cammino".

Gli esiti di quei primi due anni di intervento avevano mostrato la rilevanza di tale dispositivo, sia rispetto alla valutazione positiva, da parte dei ragazzi seguiti ma anche dei loro docenti, rispetto alla funzione relazionale e socializzante dei tutor, sia anche rispetto agli esiti scolastici. Su 117 ragazzi seguiti nel corso del primo anno di "Bussole", 107 erano stati ammessi all'esame di terza media (il 92\%), e di questi, solo uno non aveva superato l'esame. Nel secondo anno, corrispondente all'esito scolastico in prima superiore, solo il $25 \%$ di studenti seguiti era stato bocciato, e solo il $2 \%$ aveva abbandonato il percorso scolastico, per motivi peraltro riconducibili a situazioni più ampie di fragilità familiare.

\section{Molte presenze, diversi Paesi}

"Almeno una stella" è ripartito dagli esiti, positivi e incoraggianti, di "Bussole", con l'obiettivo, condiviso con la Fondazione che sosteneva il progetto, di diffondere e consolidare il modello di intervento, e di formalizzare in particolare la figura del tutor attraverso la definizione di protocolli istituzionali condivisi con le scuole e le Università aderenti al progetto. Finalità principale era continuare a sostenere i ragazzi di origine straniera nei percorsi di inclusione sociale, ma soprattutto scolastica.

Anche se con numeri più ridotti, le presenze di alunni stranieri al momento dell'avvio del progetto continuavano infatti ad aumentare sia a livello nazionale (786.630 minori, $+4,1 \%$ rispetto all'anno precedente, con un'incidenza sul totale degli alunni pari all' $8,8 \%$, contro l'8,4\% dell'anno precedente) sia nei territori coinvolti nell'intervento, con presenze significative di alunni immigrati in tutte le scuole individuate (MIUR, 2013). E come in passato, gli alunni di origine straniera continuavano a presentare esiti significativamente più fragili rispetto ai percorsi di riuscita dei pari autoctoni (si veda cap. 1).

Di seguito una panoramica della situazione regionale e provinciale, relativa alla presenza di alunni e studenti stranieri nell'anno di definizione del progetto. 
Tab. 1 - Alunni e studenti stranieri nelle scuole secondarie di primo e secondo grado nelle sei regioni considerate (a.s. 2012/13)

\begin{tabular}{|l|c|c|c|c|}
\hline Regione & $\begin{array}{c}\text { Alunni } \\
\text { stranieri } \\
\text { nelle scuole } \\
\text { secondarie di } \\
\text { primo grado }\end{array}$ & $\begin{array}{c}\text { \% sulla } \\
\text { presenza } \\
\text { totale di } \\
\text { alunni }\end{array}$ & $\begin{array}{c}\text { Studenti } \\
\text { stranieri } \\
\text { nelle scuole } \\
\text { secondarie di } \\
\text { secondo grado }\end{array}$ & $\begin{array}{c}\% \text { sulla } \\
\text { presenza } \\
\text { totale di } \\
\text { studenti }\end{array}$ \\
\hline Lombardia & 41.846 & $21,8 \%$ & 37.649 & $19,7 \%$ \\
\hline Piemonte & 15.656 & $21,2 \%$ & 15.692 & $21,2 \%$ \\
\hline Toscana & 13.927 & $22,3 \%$ & 14.847 & $23,8 \%$ \\
\hline Emilia-Romagna & 18.752 & $20,8 \%$ & 21.873 & $24,2 \%$ \\
\hline Trentino Alto Adige & 3.558 & $20,6 \%$ & 3.107 & $18,0 \%$ \\
\hline Friuli Venezia Giulia & 3.822 & $20,6 \%$ & 4.203 & $22,6 \%$ \\
\hline
\end{tabular}

Fonte: MIUR

Tab. 2 - Presenze di alunni di origine straniera nelle scuole secondarie delle province coinvolte, al momento dell'attivazione del progetto (a.s. 2012/2013)

\begin{tabular}{|l|c|c|c|c|c|c|}
\hline Territorio & $\begin{array}{c}\text { Ordine } \\
\text { di scuola }\end{array}$ & $\begin{array}{c}\text { Alunni } \\
\text { stranieri }\end{array}$ & $\%$ & $\begin{array}{c}\text { Ordine } \\
\text { di scuola }\end{array}$ & $\begin{array}{c}\text { Alunni } \\
\text { stranieri }\end{array}$ & $\%$ \\
\hline Milano & $\begin{array}{c}\text { Secondaria } \\
\text { di I grado }\end{array}$ & 15.317 & $14,0 \%$ & $\begin{array}{c}\text { Secondaria } \\
\text { di II grado }\end{array}$ & 14.849 & $10,4 \%$ \\
\hline Arezzo & $\begin{array}{c}\text { Secondaria } \\
\text { di I grado }\end{array}$ & 1.385 & $15,2 \%$ & $\begin{array}{c}\text { Secondaria } \\
\text { di II grado }\end{array}$ & 1.788 & $11,7 \%$ \\
\hline Bologna & $\begin{array}{c}\text { Secondaria } \\
\text { di I grado }\end{array}$ & 3.929 & $15,5 \%$ & $\begin{array}{c}\text { Secondaria } \\
\text { di Il grado }\end{array}$ & 3.849 & $11,4 \%$ \\
\hline Trenino & $\begin{array}{c}\text { Secondaria } \\
\text { di I grado }\end{array}$ & 7.538 & $12,2 \%$ & $\begin{array}{c}\text { Secondaria } \\
\text { di II grado }\end{array}$ & 8.332 & $9,7 \%$ \\
\hline Udine & $\begin{array}{c}\text { Secondaria } \\
\text { di I grado }\end{array}$ & 1.992 & $11,8 \%$ & $\begin{array}{c}\text { Secondaria } \\
\text { di Il grado }\end{array}$ & 1.654 & $7,6 \%$ \\
\hline $\begin{array}{c}\text { Secondaria } \\
\text { di I grado }\end{array}$ & 1.451 & $15,9 \%$ & $\begin{array}{c}\text { Secondaria } \\
\text { di II grado }\end{array}$ & 1.755 & $12,0 \%$ \\
\hline
\end{tabular}

Fonte: MIUR

Nei territori provinciali coinvolti nel progetto la presenza di alunni stranieri nelle scuole secondarie di I grado variava dall' $11,8 \%$ di Trento sino al 15,9\% di Udine. In termini assoluti i numeri più alti si registravano a Milano e provincia (15.317 studenti) e Torino e hinterland (7.538). Una presenza fortemente significativa, confermata anche nelle scuole secondarie di secondo grado, con percentuali che variavano dal 7,6\% all'12\%, e con valori di 14.849 unità a Milano e provincia (10,4\%), 8.332 studenti a 
Torino e provincia $(9,7 \%), 3.849$ a Bologna (11,4\%). Numeri estremamente significativi, spesso concentrati in alcune scuole "polarizzate", non sempre dotate delle sufficienti risorse per poter intervenire in modo efficace e sistematico.

Era quindi necessario continuare a prevedere sui territori attività mirate curate da operatori esperti di L2 e di integrazione scolastica ed educativa, con un'attenzione specifica sul momento di maggior fragilità (in uscita dalla terza media e nel primo anno della scuola superiore), e con l'obiettivo di contrastare i possibili rischi di abbandono e dispersione scolastica.

Per fare ciò, oltre a prevedere, come nel precedente progetto, la messa a disposizione di figure professionali competenti e specialiste, fondamentale è sembrata l'implementazione della presenza e del ruolo dei tutor già sperimentati in "Bussole". Figure di riferimento positive, di sostegno e di aiuto allo studio.

Il progetto intendeva inoltre ampliare lo scambio tra esperienze nazionali, allargando il confronto anche ai territori di Trento, Udine e Pordenone. L'Università di Udine, la Regione Friuli Venezia Giulia e la Provincia di Trento hanno aderito al progetto come partner istituzionali, proponendo un consolidato modello di condivisione tra enti e scuole del territorio nei percorsi di integrazione dei ragazzi di origine straniera.

Infine, si voleva sperimentare ulteriormente la sostenibilità del dispositivo, verificando l'economicità e l'efficacia dell'individuazione di figure in formazione, quindi con competenze professionali specifiche in corso di acquisizione, ma "gratuite" e volontarie, da affiancare ai professionisti in contesti inclusivi. La continua e ormai endemica riduzione delle risorse destinate a livello territoriale ai percorsi di integrazione come a tutti i progetti di prevenzione impongono infatti la necessità di pensare a modelli di intervento che siano contemporaneamente efficaci e sostenibili nel tempo.

\section{Gli obiettivi di "Almeno una stella"}

Il progetto si poneva quindi molteplici e differenziati obiettivi:

A livello scolastico:

- facilitare l'acquisizione dell'italiano L2 in particolare negli studenti neoarrivati;

- sostenere i percorsi di inserimento scolastico nella terza media e il passaggio nel primo anno di scuola superiore;

- aiutare nell'acquisizione del metodo di studio;

- contrastare i fenomeni di dispersione e abbandono;

- affiancare nella riuscita all'esame di terza media;

- facilitare la scelta della scuola secondaria di secondo grado. 
A livello socio-relazionale:

- offrire riferimenti positivi e di identificazione nei percorsi di inclusione degli adolescenti;

- accompagnare la creazione di legami relazionali con le figure adulte di riferimento, didattiche ed educative;

- facilitare la relazione con i pari;

- sostenere lo scambio interculturale e tra culture e provenienze diverse.

A livello istituzionale:

- implementare il dispositivo di tutoring, affinandone le funzioni e l'intervento;

- diffondere la figura del tutor in nuovi territori;

- rendere il dispositivo maggiormente riconosciuto e "istituzionale", formalizzando gli interventi con accordi e convenzioni nelle scuole e nelle università aderenti;

- sperimentare ulteriormente la sostenibilità del modello, ai fini della disseminazione.

\section{Quattro territori più due}

Il progetto si proponeva di ampliare la sperimentazione della figura del tutor a nuovi territori. Oltre a Milano, città capofila, Torino, Bologna ed Arezzo, si sono aggiunti la città e la provincia di Trento e il Friuli Venezia Giulia, in particolare con le province di Udine e Pordenone.

Ciascuna città, in base alle esigenze specifiche e ai percorsi di integrazione scolastica e territoriale, ha declinato gli interventi dei due anni distribuendoli nel passaggio dalla scuola media a quella secondaria di secondo grado, o concentrandoli nel biennio della scuola superiore. I tutor potevano essere o studenti delle superiori, che seguivano i loro compagni più piccoli (come nel caso di Bologna), o studenti universitari, in particolare di facoltà umanistiche (scienze dell'educazione, pedagogia, psicologia...), che hanno svolto attività di tirocinio o di laboratorio nell'ambito del loro percorso di studi.

\section{Tab. 3 - Gli enti coinvolti nel progetto}

\footnotetext{
Le città di "Almeno Una Stella" e gli enti coinvolti

Milano - Centro COME, Farsi Prossimo ONLUS S.c.s. (ente capofila)

Arezzo - OXFAM Italia, in collaborazione con il Centro di Documentazione Città di Arezzo

Bologna - CD-LEl Comune di Bologna, Settore Istruzione, in collaborazione con CIOFS FP

Torino - Centro Interculturale della Città di Torino, in collaborazione con Associazione ASAl (Associazione di Animazione Interculturale)

Trento - Provincia Autonoma di Trento, Centro interculturale Millevoci, Istituti Scolastici della Comunità Alta Valsugana e Bersntal e della Comunità Valsugana e Tesino

Friuli Venezia Giulia - Regione Friuli Venezia Giulia, Istituti di Udine, Latisana (UD), Monfalcone (GO) e Pordenone
} 
A Milano, città capofila, il progetto sosteneva i percorsi scolastici dei minori stranieri neoarrivati inseriti nelle terze medie, e a seguire nel primo anno di secondaria di secondo grado. I tutor provenivano tutti da percorsi accademici e universitari. La scelta è stata quindi di individuare come partner privilegiati l'Università Statale di Milano Bicocca (con le Facoltà di Scienze della Formazione; Scienze della Formazione - Comunicazione Interculturale; Scienze dell'Educazione; Psicologia dello sviluppo e dei processi educativi) e l'Università Statale di Milano (con le Facoltà di Scienze della Mediazione linguistica e culturale e Lingue e Culture per la cooperazione internazionale).

La collaborazione con l'Università si è formalizzata in particolare con l'Università Statale, che in un Consiglio di Facoltà ha deliberato l'assegnazione di ben 6 crediti formativi ai ragazzi che avrebbero completato il percorso di affiancamento ai minori stranieri. Presso l'Università di Milano Bicocca, invece, l'esperienza è stata riconosciuta come laboratorio interno alla cattedra di Pedagogia Interculturale.

Altri tutor inseriti nel progetto provenivano invece dalle facoltà di Scienze linguistiche e letterature straniere dell'Università Cattolica, Giurisprudenza dell'Università di Bergamo, Lingue e culture orientali dell'INALCO (Institut National des Langues et Civilisations Orientales) di Parigi.

Per quanto riguarda la scelta degli istituti scolastici nei quali effettuare gli interventi, nel primo anno sono state selezionate e contattate scuole dislocate in punti diversi del territorio cittadino, che presentavano un numero estremamente significativo di alunni di origine straniera. I progetti, interni o gestiti da enti esterni, presenti negli Istituti erano insufficienti a coprire le esigenze didattiche degli alunni stranieri. È stato quindi condiviso con i Dirigenti di attivare laboratori in preparazione dell'esame di terza media, gestiti da specialisti con l'affiancamento dei tutor. Le attività si sono avviate a febbraio 2014, e prevedevano un modulo per scuola della durata di 40 ore, mono o bisettimanale a seconda delle programmazioni degli istituti. I contenuti del laboratori hanno riguardato principalmente la produzione scritta e l'esposizione orale, per sostenere il momento della prova d'esame. Per quanto riguarda la prima parte, ci si è concentrati sul potenziamento della produzione scritta, affrontando una delle tipologie di testo previste, il testo espressivo, che vede la sua realizzazione in una lettera o in una pagina di diario. Gli obiettivi di questa prima parte sono stati:

- comprendere le idee principali di un testo facilitato;

- identificare parole chiave, lessico specifico e strutturazione di un testo espressivo e saperle riutilizzare;

- saper descrivere esperienze passate e speranze future personali;

- produrre un testo espositivo in base al proprio livello di interlingua. 
Per quanto concerne invece la preparazione all'orale, durante il laboratorio i ragazzi hanno individuato gli argomenti d'esame e sono stati aiutati nella stesura di una mappa concettuale o tesina. In particolare, sono stati supportati nella ricerca delle informazioni, nella costruzione di una presentazione informatica da esporre in sede d'esame e nella rielaborazione orale. Queste le finalità:

- ampliare il lessico grazie all'utilizzo di termini specifici;

- organizzare le informazioni e saperle riutilizzare seguendo un ordine logico e cronologico;

- approfondire alcuni argomenti disciplinari;

- acquisire maggiore padronanza all'orale;

- interagire con un interlocutore, simulando anche un'interrogazione.

I 38 alunni promossi nella prima annualità del progetto hanno individuato diverse scuole su tutto il territorio di Milano per proseguire gli studi. Per evitare quindi una dispersione eccessiva, sono state individuate quattro scuole superiori nelle quali risultava il maggior numero di iscrizioni da parte degli alunni coinvolti l'anno precedente, distribuite in zone diverse della città, in modo da poter essere raggiunte anche da alunni frequentati altri istituti cittadini. Inoltre i laboratori sono stati ampliati agli studenti neoarrivati ed inseriti nel biennio, non partecipanti in precedenza al progetto "Almeno una stella" ma iscritti nei quattro istituti secondari di secondo grado di riferimento. Le sedi individuate per i laboratori sono state: l'IIS Bertarelli, l'ITI Feltrinelli, l'IIS Maxwell/IPSIA Settembrini, l'ITE Zappa/IIS Cremona, da sempre connotati a livello cittadino da una forte presenza di ragazzi di origine straniera. Ad esempio, al Bertarelli l'attuale percentuale di studenti stranieri è pari al $43 \%$, di cui il $68 \%$ neoarrivati nell'attuale anno scolastico. Al Feltrinelli la percentuale è del 19\%. Così anche al Maxwell, che conta un 19\% di studenti di origine immigrata; ma l'IPS Settembrini, scuola limitrofa sotto la stessa dirigenza, arriva al $45 \%$. Allo Zappa/Cremona la presenza di studenti di origine straniera è del $45 \%$ all'ITE e dell'11\% al Liceo.

I laboratori nelle scuole superiori sono stati realizzati dal mese di ottobre 2014 fino a maggio 2015. Per ogni lezione è stato previsto un momento iniziale di accoglienza dei ragazzi con brevi attività ludiche per tutti ed una successiva suddivisione in piccoli gruppi per le attività di L2 e di aiuto allo studio.

Per quanto riguarda il lavoro di prima alfabetizzazione, il docente ha individuato gli argomenti da trattare (in base alle abilità registrate tramite un test d'ingresso) e ha predisposto le attività da far svolgere agli studenti con la sua supervisione e il supporto dei tutor. Le finalità sono state le seguenti: 
- acquisire un lessico minimo di base, al fine di permettere l'approccio alla nuova lingua;

- servirsi dei termini conosciuti in semplici contesti di tipo funzionale;

- usare frasi per favorire gli scambi comunicativi;

- utilizzare la struttura di base della frase usando le voci e i tempi verbali più ricorrenti.

Per ciò che concerne l'aiuto allo studio, ciascun ragazzo ha individuato di volta in volta le materia per cui era maggiormente necessario il supporto del tutor e, insieme a lui, ha svolto i compiti assegnati dai docenti di classe. Anche in questo caso i laboratori avevano cadenza settimanale, nel pomeriggio, per una durata di due ore o due ore e mezza a incontro.

Obiettivi principali dell'attività di sostegno allo studio sono stati:

- mettere a disposizione dei ragazzi uno spazio "accompagnato" e dedicato in cui affrontare insieme le criticità legate allo studio;

- facilitare e sostenere la capacità di trovare e utilizzare risorse e strumenti adeguati, anche attraverso la condivisione con i compagni e l'aiuto reciproco;

- sviluppare l'acquisizione di una corretta metodologia di studio;

- sviluppare una graduale autonomia allo studio.

Nella maggior parte dei casi, i minori hanno scelto materie nelle quali avevano più difficoltà dal punto di vista della comprensione dei testi e della rielaborazione personale, orale o scritta.

Al termine del percorso, sono state consegnate ai consigli di classe griglie di valutazione contenenti le osservazioni rilevate dai docenti e dai tutor durante gli incontri, al fine di fornire indicazioni chiare sul lavoro svolto dai singoli alunni durante il laboratorio di progetto.

Il secondo anno è stato particolarmente delicato rispetto alla valutazione della continuità dei minori. Molti sono i fattori che portano i ragazzi a disperdersi o a non confermare la disponibilità a proseguire gli interventi di accompagnamento: maggior carico di compiti scolastici nel pomeriggio, nuovi impegni o attività dopo la scuola... Ciò vale in misura ancora maggiore per chi entra nelle superiori.

Complessivamente hanno partecipato al progetto 129 ragazzi, dei quali 68 sono risultati frequentanti con regolarità. Di questi, solo 11 hanno partecipato alle attività anche nell'anno precedente; 57 sono invece stati segnalati nella seconda annualità dagli istituti superiori. 36 hanno abbandonato per motivi diversi tra cui, in particolare, la frequenza ad altri laboratori di italiano L2 organizzati nelle scuola secondarie di secondo grado prescelte, o l'impossibilità di conciliare gli orari scolastici di rientro pomeridiani con gli orari dei laboratori. 
A Torino il progetto si è realizzato principalmente presso il Centro Interculturale del Comune di Torino, in un contesto extrascolastico che ha caratterizzato l'intervento locale, rispetto a quello delle altre città partner. I partner hanno scelto di sostenere i percorsi di ragazzi inseriti nel biennio della scuola secondaria di secondo grado, partendo quindi con gli interventi nel primo anno della scuola superiore. La formalizzazione della collaborazione con le Università torinesi si è basata sulla Convenzione già in atto tra il Comune e l'Università degli Studi di Torino per la realizzazione di tirocini in progetti dell'Amministrazione locale. È stato quindi possibile riconoscere come tirocinio formativo l'intervento attivato per otto tutor, studenti dei Corsi di Laurea di Comunicazione Interculturale e Scienze Internazionali. Gli altri tutor sono stati individuati da bacini diversi (volontari del Centro, ragazzi con esperienza di servizio civile, partecipanti ai corsi per animatore interculturale del Centro, membri di associazioni di stranieri).

Per gli interventi con i minori, nella prima annualità si sono presi inizialmente contatti con le scuole secondarie di secondo grado della Circoscrizione 6 (Barriera di Milano), in cui ha sede il Centro Interculturale, quartiere con il maggior numero di residenti stranieri in città. A fronte di un forte bisogno di accompagnamento espresso da dirigenti scolastici e insegnanti, tuttavia, non è sempre corrisposta una presenza reale dei ragazzi durante gli appuntamenti al Centro, probabilmente per la difficoltà a spostarsi. Sono stati quindi attivati ulteriori contatti con scuole ad alta presenza di allievi immigrati, al di là dell'area specifica della Circoscrizione 6. Le scuole coinvolte nel primo anno sono state quindi nove: Istituto professionale di stato D. Birago; Istituto professionale di stato J.B. Beccari; Istituto tecnico statale Russell-Moro; Istituto di istruzione superiore A. Einstein; Istituto di istruzione superiore Bodoni; Liceo statale G. Bruno; Scuole professionale San Carlo; Scuola professionale Rebaudengo; Istituto tecnico Arduino. Le attività hanno riguardato laboratori L2, preparazione all'esame di terza media, doposcuola presso la sede del Centro e attività animative e di conoscenza della città.

Per la seconda annualità di progetto è proseguito il tutoraggio degli studenti promossi e inseriti al secondo anno e di quelli non promossi, frequentanti nuovamente il primo anno. A loro si sono aggiunti nuovi studenti che non avevano partecipato alla prima annualità.

In una fase iniziale sono emerse alcune criticità rispetto all'effettiva partecipazione e al coinvolgimento degli studenti ai momenti di studio, che venivano realizzati in contesto extrascolastico, presso la sede del Centro. Grazie alla continua motivazione da parte dei tutor e all'organizzazione di attività aggregative per favorire la creazione del gruppo, i ragazzi hanno invece poi frequentato assiduamente il Centro, che soprattutto nel secondo anno di progetto è diventato un importante luogo di riferimento. 
Particolare importanza è stata data al momento dell'iscrizione alla seconda annualità, che si è svolta alla presenza del ragazzo e di un genitore, al fine di stipulare un patto formativo per definire gli obiettivi del progetto. Sono inoltre stati realizzati incontri con le scuole al fine di presentare una relazione sulle attività svolte. I docenti referenti hanno espresso pareri postivi in merito, sottolineando come ci siano stati dei miglioramenti effettivi nel percorso scolastico dei ragazzi seguiti dai tutor e proposto nuovi inserimenti. I laboratori di Italiano L2 hanno avuto come obiettivo il consolidamento della lingua per comunicare, e la facilitazione dei testi delle materie scolastiche con obiettivi centrati sull'italiano per lo studio e sul potenziamento della produzione scritta e delle abilità complesse.

A questi laboratori si sono affiancati diversi momenti di accompagnamento alla conoscenza del territorio mediante attività differenti quali cineforum, passeggiate condotte da guide migranti, laboratori di teatro.

Ad Arezzo il progetto si è articolato tenendo conto della situazione di forte distribuzione nelle scuole degli studenti stranieri. Si è quindi scelto di creare due differenti gruppi di tutor: un primo gruppo costituito da studenti universitari o recentemente laureati, un secondo gruppo formato da studenti delle ultime classi delle scuole superiori. Tra i due gruppi sono stati programmati diversi momenti comuni di scambio e confronto. In base alle differenti caratteristiche dei tutor, si è deciso di attivare interventi differenziati sin dal primo anno di progetto: laboratori per il sostegno all'esame di terza media, e laboratori per il biennio delle superiori. Nei primi, il facilitatore è stato affiancato dai tutor universitari o recentemente laureati, nei secondi hanno operato sia gli studenti tutor "esterni" alla scuola, sia gli studenti tutor della scuola stessa.

Per quanto riguarda il rapporto con le Università, sono state contattati due Atenei toscani: l'Università di Firenze, Scienze della formazione primaria e l'Università di Siena, Dipartimento Scienze politiche indirizzo sociale con sede a Siena e Dipartimento Scienze dell'educazione e della formazione con sede ad Arezzo. È stato però possibile stipulare convenzioni solo tra Oxfam Intercultura e l'Università di Siena, al fine di accogliere studenti tirocinanti da coinvolgere come tutor nel progetto.

Gli altri tutor facevano tutti parte dell'associazionismo del territorio, o erano ex studenti delle scuole secondarie di II grado o delle Università.

Per quanto attiene gli interventi scolastici, nel primo anno sono stati coinvolti 5 Istituti Comprensivi e sono stati organizzati due gruppi di lavoro diversificati:

- un laboratorio di sostegno all'esame di terza media di 40 ore localizzato nella scuola; 
- un laboratorio di sostegno all'esame di terza media di altre 40 ore è stato attivato presso la Casa delle Culture di Arezzo e raccoglieva gli alunni di varie scuole.

I laboratori avevano cadenza settimanale e contavano la presenza del facilitatore linguistico esperto, affiancato dai tutor. A gennaio 2014 ha inoltre preso avvio anche il doposcuola rivolto agli alunni del biennio delle scuole secondarie di II grado coinvolti nel progetto. Le scuole coinvolte sono state: l'Istituto d'Arte Pier della Francesca e l'Itis Galilei. I doposcuola, di 80 ore a cadenza bisettimanale, sono stati gestiti da un facilitatore esperto, affiancato dai tutor.

Anche per il secondo anno la principale azione svolta è stata il supporto scolastico e l'accompagnamento degli alunni adolescenti stranieri nelle scuole secondarie e nei laboratori di sostegno allo studio, realizzati sia alla Casa delle culture che presso la sede della scuola superiore di Arezzo.

A Bologna i tutor sono stati scelti tra gli studenti del terzo e quarto anno delle scuole superiori; l'azione di sostegno linguistico-didattico e tutoraggio si è concentrata nel biennio delle secondarie di secondo grado. L'individuazione dei tutor è avvenuta prioritariamente all'interno degli stessi Istituti in cui si trovavano i ragazzi da seguire. Questa scelta si è resa necessaria soprattutto per garantire il massimo di continuità negli abbinamenti tra tutor e ragazzi tutorati per i due anni della durata del progetto, nelle scuole superiori bolognesi caratterizzate dalle più alte presenze di alunni stranieri. Per gli interventi didattici, nel primo anno sono stati coinvolti 6 Istituti Superiori della città: Aldini, Adrovandi-Rubbiani, CrescenziPacinotti, Rosa Luxemburg, Laura Bassi, Sabin, in cui sono stati avviati corsi di italiano L2 di base e A1/A2, laboratori di sostegno disciplinare e affiancamento nei corsi di recupero attivati nei vari Istituti. Per la formalizzazione dell'adesione delle scuole, sono state predisposte specifiche lettere di adesione al progetto e l'inserimento del dispositivo di tutoraggio nel POF.

Questa tipologia specifica di tutoring ha permesso ad un gruppo motivato e selezionato di ragazzi stranieri di sperimentarsi in ruoli complessi e di rilevanza sociale che richiedevano discrete dosi di impegno, autonomia e assunzione di responsabilità all'interno della scuola frequentata.

A Trento il progetto è stato realizzato grazie al cofinanziamento nel primo anno della Provincia di Trento, e ai contributi dell'Istituto di Istruzione "M. Curie" di Pergine Valsugana e Levico, e delle altre scuole aderenti. Nel secondo anno è stato autofinanziato dagli istituti, e prevedeva il coinvolgimento di tutor provenienti dall'Università degli Studi di Trento 
(Psicologia e scienze cognitive, Sociologia, Studi internazionali, Lettere e filosofia, Scienze della formazione), che hanno affiancato gli alunni stranieri delle scuole individuate in percorsi di sostegno scolastico o in attività aggregative presso centri del territorio nel periodo pomeridiano. La formalizzazione della collaborazione con l'Università si è basata sulla convenzione generale, valida per ogni facoltà e tipologia di tirocinio, già in atto tra la PAT e i diversi atenei. Il progetto è stato implementato in una rete di otto istituti scolastici e formativi distribuiti in una vasta zona geografica che comprende due Comunità di Valle: la Comunità Alta Valsugana e Bersntal e la Comunità Valsugana e Tesino. Il territorio coinvolto ha conosciuto un aumento demografico superiore rispetto al resto della regione, in particolare a causa della presenza di famiglie e lavoratori di origine immigrata. Come già per Torino, la particolarità del progetto trentino ha riguardato anche l'approfondimento degli aspetti di conoscenza e accompagnamento ai servizi socio-educativi ed aggregativi del territorio. Si è inoltre lavorato in specifico sulla figura dei "tutor di resilienza", in grado di affiancare, sostenere e motivare i minori in eventuali momenti di disorientamento o riorientamento, grazie ad una particolare e specifica formazione in ingresso.

Nel secondo anno si è valutato di rendere ancora più attivi sia i tutor, sia soprattutto i ragazzi seguiti, sostituendo i progetti di tutoraggio, con una co-costruzione tra tutor e tutorato di un "patto" controfirmato da entrambi e dai genitori del ragazzo seguito. Nel patto venivano negoziati e condivisi i tempi, i luoghi e, soprattutto, le attività da compiere assieme, così da evidenziare la presa di responsabilità da parte del tutorato e la necessità di una costanza nella frequenza agli incontri, che venivano svolti in tempo extrascolastico e, per la particolarità del contesto trentino interessato, in situazioni non strutturate.

Per quanto riguarda il Friuli Venezia Giulia, il progetto è stato finanziato dalla Regione e ha riguardato interventi realizzati nella provincia di Udine, in particolare nel comune di Latisana, Pordenone, e Gorizia e nell'area della Bassa Friulana, a ridosso del Comune di Lignano Sabbiadoro. Il progetto è stato realizzato con la collaborazione dell'Università di Udine, che ha riconosciuto gli interventi dei tutor come attività di tirocinio. Le scuole aderenti al progetto nel secondo anno sono state: gli Istituti Comprensivi Porcia e Pordenone Sud, gli ISIS Flora e Zanussi e il Liceo Grigoletti di Pordenone; l'Istituto Comprensivo Giacich di Monfalcone (Gorizia); gli Istituti Comprensivi II, III, V e gli ISIS Malignani e Stringher di Udine. L'iniziale scuola capofila del progetto, l'Istituto Comprensivo V di Udine, è stata poi sostituita dal I.C. II Di Udine.

In sintesi, complessivamente sono stati coinvolti nell'anno scolastico 2013/14 sette Istituti comprensivi (2 in provincia di Pordenone, 1 in provin- 
cia di Gorizia, 4 in provincia di Udine); nell'a.s. 2014/15 sei istituti comprensivi ( 2 in provincia di Pordenone, 1 in provincia di Gorizia, 3 in provincia di Udine) e cinque istituti scolastici di secondo grado (1 in provincia di Udine e 4 in provincia di Pordenone).

Il gruppo di lavoro è stato costituito dai docenti provenienti da I.C. e Istituti secondari della regione. Il progetto si è proposto di promuovere il tutoring come dispositivo di accoglienza per i ragazzi neoarrivati in una rete di scuole della regione, di diffondere il Quaderno dell'Integrazione del Friuli Venezia Giulia e di accompagnare le scuole, nella forma della ricerca azione, per il consolidamento di buone pratiche di intervento nelle classi eterogenee.

Studenti eccellenti (italofoni e non) della scuola secondaria di secondo grado hanno seguito ed accompagnato, in un percorso di recupero nelle materie di base, i compagni di origine straniera del terzo anno della scuola secondaria di primo grado affinché colmassero le lacune e potenziassero le proprie conoscenze per giungere preparati al traguardo dell'esame conclusivo. Il progetto di tutoraggio è stato realizzato prioritariamente in ambito didattico: è stata data priorità al recupero nelle discipline poiché i ragazzi interessati avevano già operato le scelte in merito all'orientamento scolastico ed erano (ad esclusione di una studentessa neo-arrivata) già integrati nel tessuto territoriale.

Nei due anni di progetto inoltre sono state realizzate attività di coordinamento e di rete: formazione e consolidamento del gruppo di coordinamento, allargamento e consolidamento della rete di scuole, intensificazione della collaborazione con l'USR, consolidamento della collaborazione con l'Università di Udine, ricerca di nuovi partner universitari, consolidamento della collaborazione con la Regione Friuli Venezia Giulia; attività di produzione di materiali (protocolli, materiali per il monitoraggio della situazione degli studenti, materiali di supporto per lo svolgimento dell'esame conclusivo del $1^{\circ}$ ciclo di istruzione) e orientamento al sistema scolastico per studenti e famiglie.

\section{II modello organizzativo}

"Almeno una stella" prevedeva un impianto organizzativo condiviso, con l'attivazione di momenti di coordinamento trasversali e di interventi similari, declinati, come abbiamo visto, secondo le specificità dei vari ambiti locali.

Date le finalità del progetto, per tutti i territori erano infatti previsti interventi a sostegno dell'integrazione scolastica, quali: 
- laboratori di italiano L2, per la comunicazione o lo studio, in orario scolastico e all'interno degli istituti coinvolti;

- laboratori di italiano L2, per la comunicazione o lo studio, in orario extrascolastico presso le sedi degli enti partner;

- doposcuola e attività di aiuto allo studio in orario pomeridiano, presso le scuole o le sedi degli enti;

- laboratori per la preparazione all'esame di terza media;

- attività educative e di socializzazione tra i pari, laboratori creativi ed animativi;

- attività ludiche e uscite per il periodo estivo;

- attività di conoscenza del territorio e dei servizi socio-educativi del quartiere e della città.

A livello trasversale, erano inoltre previste alcune azioni comuni, tali da permettere la confrontabilità e il monitoraggio delle azioni:

- creazione del gruppo di lavoro;

- coordinamento degli interventi;

- contatti con gli istituti scolastici e le università per la definizione delle collaborazioni;

- selezione e formazione dei tutor;

- formalizzazione degli interventi;

- momenti di confronto, scambio e verifica;

- definizione e utilizzo degli strumenti operativi e di raccolta e analisi dei dati;

- valutazione degli esiti.

A cadenze periodiche, i gruppi di lavoro degli enti partner si sono ritrovate in una delle città aderenti, per confrontarsi direttamente sull'andamento e sugli esiti. Ogni sei mesi era inoltre prevista una rendicontazione quali-quantitativa sullo stato di avanzamento degli interventi.

\section{Riferimenti bibliografici e sitografia}

Dalla Zuanna G., Farina P., Strozza S. (2009), Nuovi italiani. I giovani immigrati cambieranno il nostro Paese?, Il Mulino, Bologna.

MIUR e Fondazione ISMU (2014), Alunni con cittadinanza non italiana. Tra difficoltà e successi, rapporto nazionale a.s. 2013/2014, MIUR.

OECD, Education at a Glance. OECD Indicators (2012, 2013, 2014), OECD.

Rete europea Eurydice, www.indire.it/eurydice, studi a carattere comparativo su tematiche inerenti i sistemi scolastici europei.

Favaro G. (a cura di) (2013), Bussole sul cammino, Centro COME, Milano. 


\title{
3. Ricominciare da capo. Adolescenti che migrano e inserimento scolastico
}

\author{
di Graziella Favaro
}

\begin{abstract}
La formazione è un cammino di erranza, discontinuità, incontro, rottura. C'è sempre nel cammino di una vita una caduta da cavallo, un incontro con la terra, un faccia a faccia con lo spigolo duro del reale. Chi non si è mai perduto non sa cosa sia ritrovarsi. Ma è fondamentale la presenza degli adulti perché questo avvenga. Sono necessari una casa, un legame, un'appartenenza perché l'erranza dia i suoi frutti.
\end{abstract}

M. Recalcati, 2010

\section{Ragazzi che attraversano i confini}

Vi sono ragazze e ragazzi che frequentano le nostre scuole e che sperimentano ogni giorno sulla propria pelle i due significati del termine errare - al tempo stesso, erranza ed errore - che Massimo Recalcati esplora nel suo testo "Cosa resta del padre". Sono coloro che hanno vissuto e vivono la condizione dell'erranza che si origina dalla migrazione, dallo strappo, dalla perdita e rottura dei legami, dalla fatica del dover ricominciare ad apprendere altrove, in un'altra scuola e in un'altra lingua. Essi incontrano spesso lungo il loro cammino scolastico anche l'errore, e cioè l'insuccesso, lo scacco, l'essere e il sentirsi in disparte, le decisioni sbagliate o poco efficaci che segnano talvolta l'inizio della loro nuova scolarità. E questo fa sì che talvolta gli entusiasmi si dissolvano, le attese si appannino e si trasformino in delusioni e perdita di motivazione. Certamente, riprendendo ancora Recalcati, "l'infanzia e l'adolescenza sono il tempo in cui il fallimento dovrebbe essere consentito perché è uno zoppicamento salutare dell'efficienza e della prestazione". Ma affinché l'erranza e l'errore non diventino eventi dolorosi e annichilenti, è necessario che, accanto agli adolescenti che provano e riprovano, ci siano figure efficaci che fanno da sostegno e riferimento, da bussola e stella. "Sono necessari una casa, un legame, un'appartenenza perché l'erranza dia i suoi frutti", afferma ancora Recalcati.

Non sempre, il cammino scolastico dei ragazzi della migrazione avviene entro condizioni di sufficiente stabilità e sicurezza, supportato da fili 
affettivi di protezione e attenzioni adeguate. "L'intelligenza deve essere riscaldata", scriveva S. Weil: se questo è vero per gli adulti, lo è ancor di più per chi, nell'età dell'adolescenza, è in cerca della propria identità tra storie, lingue e riferimenti diversi e deve potersi sentire accolto e considerato nel nuovo Paese per poter apprendere.

Come abbiamo visto nella descrizione del progetto, le azioni di "Almeno una stella" hanno cercato di accompagnare i ragazzi coinvolti durante i due anni cruciali della loro scolarità: in terza media e nei primi anni dell'istruzione superiore. Sono questi i tempi dei bilanci e delle scelte; rappresentano infatti uno snodo biografico cruciale che indirizza il presente e prefigura il futuro. Sono anche gli anni di un passaggio scolastico costellato spesso da insuccessi, perdita di motivazione, abbandoni lungo il cammino e che necessitano dunque di figure di riferimento, attenzioni nei confronti di ciascuno, spazi e tempi entro i quali poter essere ascoltati e prendere la parola.

In quale misura gli adolescenti stranieri coinvolti nel progetto "Almeno una stella" sono rappresentativi degli alunni di nazionalità non italiana inseriti nelle classi terze medie e in quelle iniziali dell'istruzione superiore? Possiamo dire che le loro storie personali e scolastiche presentano ampi tratti di corrispondenza e analogia, rispetto al più vasto numero dei loro compagni stranieri. Ma hanno anche elementi specifici che li connotano e che ne fanno un gruppo segnato da maggiori vulnerabilità.

Vediamone alcuni. Sono nella stragrande maggioranza immigrati in tempi recenti o recentissimi e hanno vissuto direttamente e nell'età adolescenziale il viaggio di migrazione; si trovano dunque a dover ricominciare da capo un cammino di apprendimento, di acquisizione della nuova lingua, di costruzione di legami amicali e affettivi, essendo privati della propria storia e di una memoria e riferimenti condivisi. Compiono questo cammino con compagni sconosciuti e spesso distanti anche per età, progetti e interessi, dato che il loro inserimento scolastico avviene in classi inferiori alla loro età anagrafica. Le ragazze e i ragazzi che hanno usufruito delle proposte di accompagnamento e tutoraggio scolastico sono stati inoltre individuati dai docenti, sia il primo anno che il secondo, fra coloro che presentavano bisogni linguistici e di apprendimento importanti e urgenti e che necessitavano quindi di un supporto specifico a scuola o fuori dalla scuola.

\section{I volti e le storie}

A differenza di altri Paesi europei caratterizzati da una maggiore omogeneità nazionale della popolazione straniera, nel nostro Paese vi è una pluralità delle provenienze e delle appartenenze: ben 191 sono le naziona- 
lità individuate fra gli alunni immigrati che frequentano le scuole italiane. La mappa "etnica" delle presenze dei minori cambia inoltre molto rapidamente e segue i flussi dei ricongiungimenti famigliari, delle nuove migrazioni, della stabilizzazione delle comunità. Nella provincia di Milano, per fare un esempio, la cittadinanza prevalente fra i minori stranieri era qualche tempo fa quella egiziana, sopravanzata poi dai filippini; gli ultimi dati vedono invece al primo posto coloro che provengono dal Perù.

La diversa origine nazionale è spesso alla base della rappresentazione e della categorizzazione sociale e culturale che il contesto di accoglienza elabora e proietta sugli adulti e sui minori e naturalmente dell'auto-rappresentazione dei ragazzi stranieri che può condizionare il loro percorso di inserimento. Vi sono gruppi penalizzati da stereotipi negativi, altri convivono con uno stigma meno marcato e sono, in un certo senso, quasi invisibili; altri ancora godono di uno stereotipo "positivo", pur se collocati sempre in posizione di subalternità e funzionalità. Queste "etichette etniche" possono essere soggette a variazioni e a cambiamenti nel tempo e dipendono da fattori diversi: gli episodi di cronaca che enfatizzano i tratti e le appartenenze, i messaggi dei media, il clima sociale di una città o di una zona. L'appartenenza a uno stesso gruppo può inoltre essere un fattore "invisibile e neutro" in una località ed essere stigmatizzata in un'altra.

Alla pluralità delle provenienze si accompagna anche la varietà dei viaggi e delle storie.

Nella fotografia di gruppo degli adolescenti immigrati possiamo distinguere tre diversi percorsi che segnano identità, progetti e condizioni di vita. Nel primo collochiamo coloro che sono nati in Italia, o giunti qui nella prima infanzia, la cosiddetta "seconda generazione" in senso stretto. Dal punto di vista giuridico, sulla base della legge sulla cittadinanza vigente fino a oggi, essi sono stranieri fino alla maggiore età (quando potranno richiedere la cittadinanza italiana e seguire un iter di accesso "privilegiato"), ma sono italiani de facto. Il loro percorso di socializzazione, acquisizione linguistica e di acculturazione è infatti avvenuto in maniera precoce entro gli spazi educativi del Paese di accoglienza. Fanno naturalmente eccezione i casi di bambini nati qui e socializzati/educati in situazioni separate e condotte a parte, che rappresentano tuttavia l'eccezione. Gli adolescenti che sono nati in Italia non hanno vissuto direttamente la migrazione, il viaggio e la fase di sradicamento e di ri-orientamento nel nuovo contesto. Alcuni di loro hanno fatto raramente visita ai contesti di provenienza della famiglia e conoscono il Paese d'origine solo attraverso le narrazioni famigliari, più o meno dense di nostalgia e rimpianto. Dal punto di vista quantitativo, questo gruppo ha dimensioni più contenute, data la relativa novità dei processi di stabilizzazione degli immigrati e della diffusione piuttosto recente delle situazioni famigliari. Vi sono presenze significative di adolescenti nati in 
Italia fra gli eritrei/etiopi, gli egiziani, i salvadoregni, i marocchini, i filippini, i cinesi: le comunità più "mature" e ormai radicate.

Il secondo gruppo comprende i minori non accompagnati, coloro che sono giunti in Italia da soli, o al seguito di sedicenti famigliari, o implicati in veri e propri traffici di minori. Sono soprattutto tre le aree di provenienza dei ragazzi soli: il Nord Africa, alcuni paesi dell'Europa dell'Est, l'Africa subsahariana. Adolescenze marginali, invisibili, negate: le condizioni di vita dei minori non accompagnati sono segnate spesso da difficoltà, miseria, vessazioni. La necessità di produrre reddito immediato e in qualunque maniera impedisce a molti la possibilità di un progetto per sé e di inserimento dignitoso che richiedono tempo, tutela e risorse.

$\mathrm{Nel}$ terzo gruppo si collocano le presenze quantitativamente più consistenti: sono le ragazze e $i$ ragazzi arrivati in Italia a 12, 13, 15 anni, in seguito al ricongiungimento della famiglia. A loro è stata applicata la metaforica definizione di "generazione 1.5" (uno e mezzo) (Rumbaut, 1994), per significare una condizione di vita sospesa tra riferimenti diversi, a metà strada fra il contesto di origine e il luogo di accoglienza. Gli adolescenti ricongiunti hanno vissuto in prima persona il viaggio di migrazione, hanno sperimentato la frattura fra il "prima" e il "dopo" nella loro storia; portano con sé memoria e nostalgie, come i loro genitori, ma sono anche proiettati, come i coetanei, nei progetti e nella costruzione del futuro.

Migrare nell'adolescenza è un'esperienza cruciale che trascina con sé sfide, opportunità e alcune fatiche. Fra queste, la riduzione, almeno nella prima fase, dei percorsi di autonomia e degli spazi vitali. Ragazzi "grandi", impegnati nei contesti di origine nei processi naturali di allargamento dello spazio vitale verso l'esterno, di uscita dall'ambito famigliare per andare verso il mondo, si trovano, nel contesto di immigrazione, a ridiventare "piccoli”, a vivere in spazi più ridotti, che coincidono per un po' con la sola dimora famigliare. È un periodo più o meno lungo di blocco nel percorso identitario, che si risolverà nel momento in cui gli spazi di vita e di aggregazione saranno (se lo saranno) di nuovo molteplici e significativi: la scuola, il quartiere, i luoghi dell'incontro con i coetanei, gli spazi degli affetti e della comunità: luoghi comuni, luoghi "etnici" e luoghi meticci.

Un'altra fatica riguarda la necessità di dover ricominciare da capo in un momento della vita in cui si dovrebbe invece impegnare tutte le risorse per allontanarsi dal mondo dell'infanzia e cominciare a costruire un posto per sé. "Mi sento come un ragno", dice C., un ragazzo peruviano "che ogni giorno tesse la sua tela: ogni giorno la tela si rompe e deve ricominciare da capo".

La migrazione comporta per tutti una fase e un processo di inevitabile regressione. Non saper parlare la nuova lingua, non riuscire a esprimere stati d'animo, proposte, bisogni, scherzi, ironie, provocazioni. Non essere 
riconosciuto rispetto alla storia precedente, ai saperi e ai saper fare già acquisiti, ai livelli di autonomia raggiunti (spesso ben più ampi di quelli dei coetanei autoctoni): tutto ciò riporta a una condizione di infans (letteralmente: colui che non parla), di incapacità, di inadeguatezza.

Le riflessioni e gli interventi condotti nelle scuole e nei luoghi di aggregazione nell'ambito del progetto "Almeno una stella" si riferiscono proprio a quest'ultimo gruppo di adolescenti stranieri: nati altrove, socializzati ed educati per alcuni anni in un contesto differente, immigrati a un certo punto della loro storia e impegnati a gestire le sfide dell'adolescenza in un luogo ancora, per certi versi, estraneo e opaco.

\section{Il viaggio all'improvviso}

L'esodo che porta gli adolescenti nel nuovo Paese è per molti scandito e accompagnato da vissuti ed emozioni contrastanti: attese e illusioni nei confronti del futuro, timori e inadeguatezza di fronte ai nuovi compiti e alle situazioni inedite, perdita e nostalgia per gli affetti, gli amici, i compagni di gioco che si sono lasciati alle spalle. Un marasma emotivo che può essere più o meno intenso e destabilizzante a seconda dell'età, delle modalità della partenza e dell'arrivo, delle condizioni di accoglienza e delle forme di coinvolgimento nel nuovo progetto di vita.

La maggior parte dei minori ricongiunti non sceglie se e quando migrare. Le condizioni famigliari, i vincoli burocratici, l'accadere di eventi inattesi possono spingere il capofamiglia verso decisioni improvvise, o viceversa possono costringerlo ad una sorta di stallo decisionale e a posticipare la partenza per lungo tempo, lasciando i figli in una situazione di sospensione e di attesa senza confini certi e senza progetti.

In ogni caso, il momento del ricongiungimento della famiglia mette a confronto le aspettative e le traiettorie di vita differenti, richiede cura, attenzioni, risorse emotive e materiali per segnare il nuovo inizio della storia famigliare e non costituire soltanto un evento impregnato di nostalgia.

In quale modo i ragazzi che si ricongiungono al/ai genitore/i emigrati in precedenza vivono questo evento, segnato da sentimenti di perdita/ separazione e di attesa per il nuovo incontro? Quali sono gli elementi che possono riattivare o provocare situazioni di vulnerabilità, o che, viceversa, possono facilitare la ricomposizione affettiva e progettuale del nucleo e l'elaborazione di un nuovo modo di vivere insieme, negoziato e condiviso?

Ricominciare a vivere insieme nel paese di immigrazione rappresenta una tappa decisiva nella storia famigliare, che segna in maniera profonda un "prima" e un "dopo" e che comporta modificazioni importanti che coinvolgono piani e soggetti diversi. I cambiamenti riguardano infatti gli 
aspetti giuridici, sociali, economici, psicologici e della relazione (interna alla famiglia ed esterna, con i servizi), progettuali. Se l'immigrato singolo ha potuto continuare a vivere per anni in una sorta di invisibilità sociale, rispetto ai servizi e ai luoghi di vita per tutti, la presenza del nucleo famigliare lo costringe a entrare in contatto con i servizi, a modificare i suoi progetti, a rivedere le modalità di relazione all'interno e all'esterno della famiglia. Molti sono gli elementi che entrano in gioco nel momento del re-incontro dopo la parentesi della separazione che può essere durata anni.

Ne citiamo alcuni:

- uno spazio nuovo da suddividere in maniera diversa e da condividere;

- il progetto migratorio da riconsiderare nella sua durata, tempi, obiettivi;

- le relazioni affettive da ricostruire, a partire dai vissuti reciproci della distanza sedimentata nel tempo e di una conseguente estraneità dovuta alla lontananza protratta;

- le immagini reciproche da riconoscere e confrontare con le rappresentazioni che ciascuno aveva elaborato durante l'assenza;

- le aspettative e le illusioni da demolire o ridimensionare per far posto a nuove promesse e speranze da costruire insieme.

Sono dunque molteplici le sfide che i minori della migrazione si trovano a dover affrontare subito dopo il loro arrivo. Devono infatti ritessere fili affettivi nei confronti del genitore, o di entrambi i genitori, ai quali si ricongiungono. Questi possono essere per i figli quasi degli estranei, partiti tempo addietro e visti durante il periodo della separazione del nucleo in maniera saltuaria e in contesto differente.

Il tema dell'attaccamento e della separazione è quindi centrale nella storia di vita dei ragazzi ricongiunti. Una prima separazione dal genitore (o da entrambi i genitori) emigrato anni prima; una successiva separazione dalle figure parentali e affettive che li hanno cresciuti fino a quel momento in patria, al momento del ricongiungimento. Essi devono inoltre trovare dentro di sé risorse e motivazioni importanti e consolidate per potersi inserire nella nuova scuola, con i pari, imparare la nuova lingua, sia per comunicare nel quotidiano, sia per studiare, apprendere, riuscire. Al momento dell'arrivo nel nuovo Paese, vi possono essere inoltre altre emozioni e vissuti che accompagnano la prima fase dopo il ricongiungimento, quali: la caduta delle illusioni e delle aspettative precedenti, la perdita di prestigio dell'immagine paterna, ritenuto fino a quel momento potente, o ancora, l'impatto improvviso con situazioni famigliari e legami affettivi inaspettati e fonte di disagio (alcune donne emigrate da sole, ad esempio, ricostruiscono in Italia un nuovo nucleo).

Tutti questi aspetti del cambiamento - che hanno a che fare con le relazioni e i legami intrafamigliari, con il rapporto con la scuola, con i pari e 
il mondo esterno - sono acuiti e resi più pregnanti, se vissuti nell'età dell'adolescenza. Spesso i ragazzi ricongiunti hanno subito la decisione dei genitori o del genitore e si trovano a partire all'improvviso, senza averlo scelto o "negoziato". L'arrivo nel nuovo Paese comporta per alcuni forme di dipendenza, perdita dei legami, dei riferimenti e dell'immagine di sé, blocchi in un percorso di autonomia che sono difficili da accettare. L'inserimento scolastico in situazione di svantaggio linguistico, di ritardo rispetto alla classe e di distanza nei confronti dei pari, rende inoltre più acuto il vissuto di isolamento e di perdita.

\section{Le fatiche della migrazione}

Più che l'evocazione delle difficoltà e dei problemi, ci sembra che il concetto di sfida, riferito alla condizione di vita degli adolescenti immigrati, sia il più efficace e adeguato per tener conto, sia delle specificità, sia dei tratti comuni a tutti gli adolescenti. Le sfide, o compiti di sviluppo, segnano infatti il cammino di autonomia e di crescita di tutti i ragazzi, qualunque sia la loro origine. A queste prove, proprie del diventare grandi e della costruzione dell'identità, si aggiungono, per le ragazze e i ragazzi dell'immigrazione, altre fatiche specifiche e derivate dalla loro situazione di provvisorietà e di indefinita appartenenza (Favaro, Napoli, 2004).

Parlare di sfide ci consente quindi di descrivere tratti di specificità entro un quadro comune che contiene e definisce le adolescenze del mondo. $\mathrm{Ci}$ consente anche di rappresentare ed evocare, accanto alla descrizione dei compiti e delle difficoltà, le risorse - personali, famigliari, legate al contesto di accoglienza - che il minore mobilita per rispondere alle richieste. Una sfida è infatti una prova della vita (comune ai coetanei, o specifica dell'essere immigrato) che può essere superata, aggirata, evitata, spostata in là nel tempo. Evoca comunque un cammino aperto, ancora da compiersi, lungo il quale le riuscite e le conquiste si intrecciano con le difficoltà e gli scacchi. Parlare di sfide significa dunque tratteggiare le specificità di un cammino che è ancora in fieri, che è ancora aperto a diverse possibili uscite. Ci consente anche di non mettere l'accento - come viene fatto troppo spesso - solo sul tema del disagio, dei problemi, delle difficoltà, ma di lasciare strada alle potenzialità individuali e alle inedite risposte di ciascuno.

Presentiamo dunque alcune sfide che devono affrontare le ragazze e i ragazzi stranieri attraverso le loro parole e frammenti di storia. 


\section{Gestire le separazioni affettive}

"Mi sento solo, mi mancano i nonni, ma ogni domenica li chiamo";"Il mio amico del cuore è nelle Filippine"; "Mi mancano tanto $i$ miei fratelli che sono rimasti in Ecuador"; "Stavo bene in P. col papà, ma mi mancava la mamma, piangevo. Ci scrivevamo e ci spedivamo le foto. Passavo tutto il tempo con mia cugina, lei ha rappresentato la mia amica del cuore".

Nella storia dei ragazzi stranieri, gli episodi di separazione segnano profondamente i capitoli di un tempo popolato di pesanti assenze e di presenze "sostitutive". Tutti hanno vissuto una prima lacerante separazione durante l'infanzia a causa dell'emigrazione dei genitori: a volte di entrambi, altre volte del padre o della madre. Il loro legame di attaccamento privilegiato si è allora indirizzato verso altre figure affettive: i nonni, soprattutto, ma anche gli zii, i cugini, i parenti vari che sono stati gli spettatori delle conquiste quotidiane, dei piccoli e grandi eventi che scandiscono l'infanzia di ogni bambino. Il filo affettivo con il/i genitore/i lontano si è talvolta affievolito, ma mai interrotto: è stato riallacciato o rinsaldato attraverso le telefonate, le lettere, le fotografie e le videocassette, i regali e gli oggetti, i racconti famigliari e le attese delle visite e dei contatti.

Al momento della partenza per l'Italia, gli adolescenti hanno vissuto poi altre separazioni: dai nonni, dagli amici "del cuore", dai compagni di classe, dai luoghi, gli edifici, i paesaggi che sono stati fino a quel momento teatro della loro crescita e delle avventure quotidiane. I sentimenti di nostalgia e di mancanza coinvolgono allora i nonni, con i quali il legame era forte, quotidiano e non aveva bisogno di parole. Con gli amici del cuore pian piano i fili si allentano: "Non ci sentiamo più; non ho più il numero di telefono"; "Ho visto i miei amici l'estate scorsa in Perù, erano cresciuti, ma io sono cresciuto di più!"; "Loro mi hanno dimenticata e io li ho dimenticati”.

\section{Ritessere nuovi legami}

L'allontanamento dalle rive dell'infanzia comporta anche una presa di distanza dai legami più "antichi" e il riconoscimento delle proprie e altrui modificazioni. E vi è un ulteriore cammino di avvicinamento affettivo, che comporta fatiche, vissuti di solitudine e di estraneità, da contenere e superare. Si colloca nella prima fase dopo l'arrivo e ha a che fare con il legame di attaccamento che deve essere costruito qui giorno dopo giorno, con i genitori (il genitore) ai quali ci si è ricongiunti. "Tra me e papà c'è un muro, non riesco a spiegare" dice Y.L. che evoca invece la grande nostalgia che sente per i nonni che l'hanno cresciuta e per la mamma che ha fatto rientro in Cina. E B., che ha sofferto molto per la separazione dai genitori, ora, un anno e mezzo dopo il ricongiungimento del nucleo, dice: "Adesso vorrei 
che loro fossero in Ecuador e io in Italia, oppure, loro in Italia e io ancora in Ecuador".

\section{Sentimenti ambivalenti}

La decisione della partenza viene rievocata da quasi tutti i ragazzi come improvvisa, non elaborata: "La nonna si è ammalata e non poteva più tenermi con sé"; "In Italia sono venuta da sola, un giorno mi hanno messo sull'aereo, dove ho conosciuto una ragazza peruviana, sola come me". Un evento famigliare (la malattia della nonna), le mutate condizioni di vita in Italia, alcune inaspettate possibilità che si aprono: sono alcuni dei fattori che rendono effettiva la partenza dei figli, senza che questi spesso siano stati avvertiti e coinvolti nelle scelte. Anche per questo carattere di rottura rispetto al proprio tempo, i sentimenti che accompagnano il viaggio sono spesso ambivalenti. "Ero contenta e triste. Contenta perché rivedevo mia mamma e mia sorella; triste perché lasciavo il papà, i nonni, gli amici"; "Io non volevo venire qua perché avevo paura di non parlare subito l'italiano e non volevo lasciare i miei cugini". "La decisione di farci venire in Italia l'ha presa mio padre. Ero contento e spaventato. Contento perché raggiungevo i miei genitori che non vedevo da tanto; spaventato perché non ero mai salito su un aereo e non sapevo che cosa avrei trovato, gli amici, il nuovo". "Volevo venire per la mia mamma, però, quando ho avuto il biglietto in mano, mi sono detta: 'Nooo... cosa sto facendo? Devo rimanere qui, sto bene, la mia vita qua è divertente'...”.

\section{Il tempo diviso}

A differenza dei più piccoli che affrontano l'esodo quasi sempre con maggiore incoscienza e minor timore, i ragazzi avvertono il peso della prova, la paura di non riuscire a parlare, a inserirsi, a farsi degli amici. Il timore di non trovare il proprio posto nel mondo e di non essere accettati, che accompagna tutte le adolescenze, si amplifica e si popola di nuovi fantasmi di esclusione e di perdita. Il disegno di Y.L. esprime con forza questo vissuto di scissione e di divisione dentro di sé. Disegnando il suo presente, la ragazza rappresenta se stessa come corpo diviso in tre parti: la testa, il busto, le gambe. La testa è qui in Italia, impegnata a riuscire nello studio e nella vita ("a diventare una persona eccellente", così dice); il tronco che contiene il cuore e gli affetti è rimasto in Cina, fortemente legato alla nonna e alla mamma, che nel frattempo è tornata a vivere là; le gambe che le permetteranno di muoversi, di uscire dalla situazione attuale, di cambiare luogo e paese, indicano il cammino futuro. Per una fase abbastanza lunga, questi sentimenti ambivalenti rispetto alle scelte famigliari di ricomposizione del nucleo in Italia (e di conseguenti fratture affettive) 
restano vivi e presenti. E le difficoltà che le sfide connesse alla migrazione spesso comportano riattivano il vissuto di aver subito le decisioni altrui, di non avere scelto la rotta del proprio viaggio.

\section{Uno spazio famigliare ristretto}

Evento cruciale nella storia delle famiglie immigrate, il ricongiungimento modifica profondamente la storia di ciascuno e impone di rivedere i reciproci ruoli. Per chi è vissuto fino a quel tempo da solo (il padre, la madre, o entrambi i genitori emigrati anni prima) si tratta di gestire una ristrutturazione importante del proprio tempo, spazio, modi di vivere. Di mettere a nudo, in maniera palese e dirompente, le riuscite e gli insuccessi, le nuove capacità acquisite e le inadeguatezze. Accogliere la parte spezzata della propria famiglia nel nuovo contesto significa diventare iniziatori del loro viaggio, accompagnatori attenti sui quali si riversano aspettative e richieste. Chi arriva, da parte sua, porta con sé timori e speranze e anche le molte attese elaborate durante il tempo della distanza. Ognuno assume nei confronti dell'altro un nuovo ruolo, reso denso dalle reciproche aspettative e reso necessario dai cambiamenti intervenuti nel corso del tempo. Le rappresentazioni rigide dell'essere marito, moglie, genitore, figlio che ognuno porta con sé possono non combaciare più con la realtà prodotta dalle trasformazioni e dalle assenze. Il padre, emigrato anni prima, può avere conservato e irrigidito un modello di famiglia e di ruoli intergenerazionali all'interno di essa, che risulta ormai superato e anacronistico anche nello stesso paese d'origine. La donna, emigrata da sola e abituata da tempo a vivere un ruolo di autonomia e di indipendenza, può provare fastidio e insofferenza nei confronti di richieste più tradizionali da parte del coniuge ricongiunto.

In questo clima famigliare che aspetta di trovare un equilibrio nuovo in uno spazio fisico e relazionale divenuto più stretto per tutti, le ragazze $\mathrm{e} i$ ragazzi immigrati portano i loro bisogni e progetti, le insoddisfazioni e le riuscite, le distanze affettive e le chiusure. È soprattutto nei confronti dei padri che vengono espressi i sentimenti più ambivalenti. "Prima andava bene, andava normale come papà. Adesso non parliamo quasi, infatti non è che si parla, a volte litighiamo perché non capisce e non mi capisce"; "Proprio non mi piace essere in casa perché c'è mio padre. Perché negli ultimi tempi abbiamo litigato un sacco e allora esco sempre... Io non so come devo fare. Quando sono in casa sono triste..." Alle incomprensioni "normali" tra genitori e figli, tipiche dell'età e della fase di vita, la migrazione aggiunge complessità e vissuti di vicinanza/distanza che si rielaborano all'interno di ciascun nucleo. Anche il rapporto con i fratelli 
è segnato dall'evento migratorio. Coloro che hanno sperimentato e vissuto insieme la separazione del nucleo e la distanza dai genitori, hanno imparato a sostenersi a vicenda ("In Perù siamo rimaste per un po' di tempo solo io e mia sorella"), a trarre forza dal legame fraterno. Ma vi può anche essere una sorta di linea di separazione tra i ragazzi ricongiunti e i fratelli, più piccoli, che sono nel frattempo nati in Italia. Il modo di comunicare, l'accento, la gestualità, il modo di occupare lo spazio: sono i segni, verbali e non verbali, che definiscono la differenza delle traiettorie di vita. Tanto più se $\mathrm{i}$ fratelli nati qui sono i figli di una nuova coppia e di un altro padre. È il caso, ad esempio, di alcune donne latino-americane e filippine, che accolgono i figli rimasti fino a quel momento nel paese d'origine all'interno di una "nuova famiglia", composta da un nuovo partner e da bimbi piccoli nati nel frattempo e sconosciuti fino a quel momento ai ragazzi più grandi.

"Qui c'è la mia sorellina di un anno e mezzo che è la figlia del compagno della mamma. Aiuto la mia mamma a curare la casa e a curare la mia sorellina".

\section{6. "All'inizio non capivo niente"}

Tutti i ragazzi coinvolti nel progetto "Almeno una stella" pongono come centrali nella loro storia, nel recente passato e anche nel presente, le difficoltà linguistiche. L'apprendimento dell'italiano per riuscire a capire almeno un po' e a farsi capire nel quotidiano è stato per tutti abbastanza veloce (ha richiesto ad alcuni due/tre mesi; ad altri fino a un anno). Molto più arduo e impegnativo è l'apprendimento della nuova lingua "di studio", del lessico, delle strutture e dei significati che servono per poter seguire e comprendere i contenuti delle diverse discipline e del curricolo comune. È questo per molti un percorso ancora da esplorare, approfondire, guadagnare.

Imparare una seconda lingua nell'età della preadolescenza o dell'adolescenza presenta vantaggi e svantaggi. L'acquisizione del nuovo codice si colloca proprio nella cosiddetta "età critica" che alcuni studiosi pongono intorno ai 12/13 anni. Fino a quel momento la plasticità del cervello e dell'apparato fonatorio e di discriminazione uditiva sembrano fornire le condizioni ideali per lo sviluppo di un bilinguismo "perfetto", che tende a sviluppare una competenza fonologica del tutto simile a quella dei parlanti nativi. Dopo l'età "critica", le possibilità di recepire e di produrre suoni che non sono presenti nella propria lingua d'origine sembrano ridursi e il bilinguismo che si può sviluppare risentirebbe di questi limiti (soprattutto di tipo fonologico e ortografico). Poniamo il caso di due fratelli, arrivati per 
ricongiungimento famigliare a 6/7 anni e a 13/14 anni. Sulla base di questa ipotesi, il primo si trova nelle condizioni per poter sviluppare una competenza bilingue da nativo; il secondo potrà diventare ugualmente bilingue, ma nelle produzioni orali (e scritte?) potranno essere presenti elementi e tratti dovuti all'interferenza dalla lingua d'origine.

Imparare una seconda lingua nell'adolescenza può presentare tuttavia anche un vantaggio. I percorsi di scolarità condotti nel paese d'origine fino al momento della migrazione consentono infatti al ragazzo non italofono di consolidare la propria competenza nella prima lingua (e, a volte, anche in una lingua straniera) di saper usare il codice d'origine per lo studio, di riflettere sulle strutture e sulla grammatica. Tutte queste capacità - di tipo linguistico, cognitivo, metalinguistico - possono con il tempo trasferirsi anche nella seconda lingua, sostenere un percorso di studi positivo e, al tempo stesso, consentire il mantenimento/sviluppo di un bilinguismo più avanzato. Naturalmente questa possibilità diventa reale se la scolarità nel paese di origine è stata qualitativamente positiva e adeguata all'età.

Altri fattori - di tipo affettivo, psicologico, identitario - connotano il percorso di apprendimento di una seconda lingua nell'adolescenza. Per i più piccoli, imparare le nuove parole assomiglia ad un gioco e ha forti connotazioni di tipo ludico e spontaneo; per un giovane e per un adulto, questa sfida ha i caratteri della necessità e dell'obbligo; per i ragazzi immigrati, l'acquisizione della seconda lingua non può che essere il risultato di una "scelta" e di un desiderio. Le implicazioni affettive e psicologiche sono infatti molto più dense e hanno a che fare con il proprio progetto di futuro, con la volontà o meno di entrare a far parte del gruppo dei pari, con la motivazione profonda all'integrazione, con la rappresentazione che ciascuno ha elaborato del nuovo paese e della sua lingua.

Gli adolescenti immigrati, in Italia e in altri Paesi europei, presentano situazioni scolastiche segnate spesso da difficoltà e insuccessi. I dati raccolti annualmente dal MIUR sull'inserimento degli alunni di altra nazionalità, ai quali abbiamo accennato nel capitolo 1, indicano chiaramente alcuni punti critici. L'inserimento nella scuola italiana dei ragazzi ricongiunti rappresenta per gli insegnanti un problema emergente al quale la scuola deve dare risposta positiva. Rappresenta per gli adolescenti coinvolti una sfida complessa che, a volte è segnata da vissuti di regressione, frustrazioni, perdita di motivazione. Si deve ricominciare tutto da capo in un nuovo contesto, del quale si ignorano le regole, esplicite e implicite, non si comprendono le parole e i gesti, si fraintendono le mete e i modi di funzionamento. Per un periodo più o meno lungo, i ragazzi neoarrivati stanno in classe irrigiditi e silenziosi in attesa che gli eventi del quotidiano e le parole della scuola diventino evidenti e significativi. 
L'esperienza scolastica precedente presenta quasi sempre delle differenze importanti e non sempre aiuta a ri-orientarsi. In genere, i ragazzi immigrati hanno sperimentato modalità pedagogiche e didattiche di tipo più tradizionale e trasmissivo e modelli disciplinari espliciti, rigidamente osservati e regolati da sanzioni precise. Nella scuola italiana, le regole appaiono meno evidenti, la gestione della disciplina è meno rigida, la relazione tra gli alunni e gli insegnanti è più immediata e vicina. L'interazione tra insegnanti e alunni, ad esempio, oltre a essere di difficile comprensione per le difficoltà linguistiche, può a volte generare spiazzamento anche per le modalità comunicative adottate. In Italia spesso le consegne e le richieste sono poste in maniera "velata" e implicita e possono risultare opache. Spesso gli insegnanti esprimono gli ordini in forma di invito e di domanda, così da attenuare il valore performativo dei messaggi che tuttavia questi inviti hanno. Nella scuola del paese d'origine invece le domande di prestazione e di disciplina sono in genere espresse in modo più diretto e esplicito, poste in una forma non ambigua e non mediata.

Le reazioni nei confronti delle differenze tra i sistemi scolastici possono essere diverse. Alcuni provano disagio di fronte alla confusione, al rumore, al venir meno di segnali chiari che definiscono i tempi, le attività e i ruoli e possono rimanere in disparte e in silenzio: "I primi tempi avevo sempre mal di testa per la confusione che c'era in classe"; "Non ero abituata al disordine e non capivo che cosa fare"; "Durante le lezioni i miei compagni fanno una grande confusione, cosa che non avviene in Cina. Anche il metodo è molto diverso: là seguivano il libro con ordine, qui si salta. Io già faccio fatica a capire e con la confusione... Però ultimamente mi sto abituando...".

Altri continuano a stare in classe seguendo il modello "più tradizionale" già interiorizzato nella scuola d'origine: si concentrano sul compito più che sulle relazioni con i pari, stabilendo a volte un rapporto privilegiato con gli insegnanti, o con gli adulti in genere: "Mi hanno messo in prima media, avevo due anni più dei miei compagni. Non stavo bene con loro, ma andavo d'accordo con gli insegnanti".

Altri ancora, possono avere difficoltà a stabilire i nuovi confini, a decodificare e fare propri regole e messaggi impliciti, a comprendere la portata delle trasgressioni, distinguendo tra comportamenti ammessi e tollerati (le piccole trasgressioni che creano gruppo e legami) e comportamenti che non vengono accettati. Per questi ultimi, la nuova scuola che ha regole implicite e poco evidenti può apparire allora priva di regole e di confini e questo li porta ad assumere comportamenti non accettati. 


\section{Farsi dei nuovi amici}

"Ho un amico del cuore nelle Filippine"; "Con le amiche del mio paese posso parlare liberamente perché parliamo la stessa lingua, invece con gli italiani ci devo pensare su"; "Con le ragazze italiane, non so come mai, non riesco tanto a entrare in comunicazione, forse per la lingua"; "Mi trovo meglio con gli stranieri perché ci capiamo meglio, per un discorso di lingua e di carattere"; "Di solito stiamo insieme il filippino, il romeno e io".

Attorno al tema dell'amicizia e degli amici si concentrano i racconti più carichi di emozioni e di attese. Le amicizie che si stabiliscono durante l'adolescenza sono quelle "del cuore". Sia che proseguano anche dopo questa età, sia che si concludano in questo tempo, gli amici incontrati in questo periodo lasciano un segno indelebile. È con loro che si vivono le prove e le avventure, con loro ci si confida, si condividono paure e segreti; il loro giudizio e la loro attenzione possono confermare o ferire. La maggior parte dei ragazzi racconta di aver lasciato gli amici del cuore nel paese d'origine. A più di un anno dal momento dell'arrivo in Italia, la densità relazionale con $\mathrm{i}$ coetanei pare segnata ancora da assenze e solitudini, da nostalgia e perdita. Le ragazze soprattutto indicano le "migliori amiche" solo fra i famigliari che sono in Italia (la mamma, la sorella, le cugine...); mentre i ragazzi tendono a presentare il fatto di essere spesso soli come una scelta e una forma di autonomia. Per tutti, gli ambiti privilegiati delle possibili amicizie sono la scuola, da un lato e i momenti della festa e dell'incontro fra connazionali, dall'altro. È proprio nell'ambito degli incontri "etnici" e dell'appartenenza comunitaria che gli adolescenti immigrati collocano le loro amicizie, nascenti o consolidate. Con i ragazzi del proprio paese, o con altri stranieri, conosciuti nelle situazioni di doposcuola e di aiuto allo studio in tempo extrascolastico, sembra più facile stabilire dei legami sulla base della comune appartenenza o di esperienze condivise. La maggiore distanza nei confronti dei coetanei italiani è spiegata da tutti in termini linguistici: la scarsa competenza in italiano - non tanto per comunicare, quanto per riuscire a esprimere scherzi, ironia, allusioni, motti di spirito, immagini metaforiche... - sembra erigere una barriera quasi invalicabile che produce forme di esclusione e di auto-esclusione. È certamente una spiegazione che contiene parte della verità, ma è anche un dato "oggettivo" che talvolta nasconde forme più profonde di rifiuto e discriminazione, di auto-esclusione e di ripiego su di sé. La "differenza accettabile" nei confronti dei pari - quella esterna a sé, della non competenza in italiano - lascia così nell'ombra le indicibili forme e i vissuti di esclusione con i quali non ci si può ancora confrontare.

Nel momento della vita in cui gli spazi del quotidiano tendono ad ampliarsi e, dalla dimora famigliare, si aprono sul mondo, la migrazione può 
comportare per gli adolescenti stranieri un restringimento dei luoghi di vita e delle relazioni. Nella loro storia appaiono centrali solo due dimensioni: quella famigliare e quella scolastica, investite di tutte le aspettative, i bisogni e le emozioni. Tra casa e scuola, pochi altri ambiti di incontro arricchiscono il panorama del tempo/spazio quotidiani. Durante i momenti di laboratorio, è stato chiesto ai ragazzi coinvolti nel progetto "Almeno una stella" di citare cinque luoghi della città (oltre alla casa e alla scuola) che conoscono e che frequentano abitualmente o saltuariamente. Nessuno ha elencato tutti e cinque i luoghi; le risposte più diffuse ne indicano due/tre e sono, per Milano: il Duomo, corso Buenos Aires, i parchi, per alcuni; l'oratorio, il CAG, il centro o l'associazione di aiuto allo studio e di doposcuola, per altri; i luoghi di incontro della comunità di appartenenza, per altri ancora. Si delineano dunque tre diversi ambiti di uso della città e di incontro:

- i luoghi pubblici (la piazza, il parco, le vie dei negozi), nei quali si sta soli in mezzo agli altri, luoghi quindi non investiti di valenze affettive o di coloritura emotiva;

- i luoghi di aggregazione e di aiuto (l'oratorio, il CAG, l'associazione, il doposcuola...) nei quali si possono incontrare i coetanei, inserirsi in attività ludiche e sportive, oppure trovare risposta e forme di sostegno a bisogni di tipo linguistico, scolastico e di apprendimento. In questi spazi, gli incontri privilegiati avvengono spesso con altri ragazzi immigrati, della stessa o di altre nazionalità;

- i luoghi "etnici" (sale di riunione, chiese, feste, occasioni di incontro della comunità...): in questi spazi si rinsaldano i legami comunitari, ci si ritrova con altri ragazzi connazionali, si rinnova l'appartenenza al paese d'origine. Nei luoghi "etnici" vi è anche la possibilità di costituire gruppi di coetanei nei quali esprimere capacità e creatività: squadre per attività sportive, bande musicali, associazioni di danza e di teatro...

Le forme di esclusione o di auto-esclusione dalla vita relazionale e dagli spazi frequentati dai pari autoctoni trascina con sé un'altra forma di esclusione, meno visibile, ma ugualmente importante: quella dalle narrazioni dei coetanei, durante le quali si rievocano, commentano, progettano... gli usi dello spazio e del tempo da condividere. Le ragazze e i ragazzi immigrati, che conducono una vita relazionale e aggregativi meno densa e spesso "parallela", non possono partecipare a questi racconti che rinsaldano i legami, strutturano i gruppi, creano amicizia. A differenza dunque dei loro coetanei italiani, per i quali la dimensione dell'incontro si collega a tempi e luoghi per lo più extrafamigliari e extrascolastici (le case degli amici, il quartiere, i centri sportivi), per i ragazzi immigrati, le relazioni si tessono e si rinsaldano solo negli spazi della scuola, della famiglia e della comunità. 


\section{Far fronte a episodi di discriminazione}

"Ho avuto qualche difficoltà con i compagni a scuola perché mi facevano gli scherzi, mi tiravano i coppini o le sberle"; "Mi prendevano in giro per come parlavo l'italiano; quando andavo a fare educazione fisica, prendevano la palla da basket e mi dicevano: - Questa è palla, si chiama palla... - Mi trattavano come un cagnolino, ma tanto io capivo perché prima di andare a scuola sono stato a casa sei mesi e ho imparato l'italiano"; "Se qualcuno vuole creare dei problemi con me, io me ne vado"; "Mi prendevano in giro e mi dicevano 'non capisci niente'. All'inizio ho fatto molta fatica, ma poi ho fatto un po' di cattiverie ai miei compagni, così capivano che non mi potevano trattare così".

Nella prima fase dopo il loro arrivo in Italia, la maggior parte dei ragazzi intervistati ha vissuto episodi piccoli o grandi di discriminazione e di rifiuto da parte dei compagni di classe, che per alcuni continuano anche dopo due anni di inserimento. Anche in questo caso le difficoltà linguistiche, l'accento, le modalità di pronuncia, la lentezza e gli impacci del discorso vengono citati come le cause scatenanti dei comportamenti offensivi o distanti dei coetanei. Di fronte alla necessità di "sopravvivere" nel gruppo dei pari e di ritrovare un posto nelle relazioni in classe, gli adolescenti immigrati possono reagire in modo diverso. Vi sono coloro che:

- cercano di restare invisibili in classe: poco coinvolti nelle reazioni con i compagni si proteggono e difendono dalle situazioni di conflitto possibile evitandole e concentrandosi sull'apprendimento, sul compito e stabilendo a volte una sorta di alleanza solo con gli insegnanti;

- accettano (almeno per una certa fase) un ruolo di subalternità e di dipendenza dai pari, i quali sottolineano in vario modo la loro non competenza linguistica, comunicativa, sociale;

- fanno valere nell'ambito del gruppo le loro capacità e abilità sportive, ludiche, musicali... e questo elemento diventa il loro punto di forza nella relazione con i pari;

- reagiscono agli episodi di discriminazione con risposte di tipo aggressivo e di scontro anche fisico.

Quest'ultimo comportamento sembra poco diffuso fra i ragazzi di recente immigrazione, i quali sembrano tendere invece verso una sorta di invisibilità nel gruppo, un comportamento di distanza auto-protettiva che dovrebbe metterli al riparo da episodi di discriminazione.

Il tema della solitudine ricorre di frequente nelle parole degli adolescenti immigrati: "Mi sento sola"; "Sto sempre da solo"; "Mi piace stare da solo, sono di carattere timido e chiuso". Un numero minore di relazioni amicali con i pari, gli amici "del cuore" per lo più collocati nel paese d'origine, un 
tempo/spazio extrascolastico ed extrafamigliare meno denso affettivamente: sono alla base dei vissuti diffusi di solitudine e di "vivere in disparte". Una certa distanza affettiva si sperimenta anche nei confronti dei genitori, o di uno di essi: nei confronti di colui che è immigrato anni prima e che è, per certi versi, una sorta di estraneo; nei confronti del padre, più che della madre; nei confronti di fratelli nati in Italia e sconosciuti fino al momento del ricongiungimento famigliare. La solitudine accompagna anche il confronto quotidiano con le sfide che la situazione di ri-orientamento comporta. Gli adolescenti immigrati hanno così spesso l'impressione di "farsi da soli", di diventare demiurghi di se stessi, dal momento che i loro genitori non possono sostenerli nell'affrontare compiti e prove di tipo linguistico, scolastico, relazionale (Beneduce, 1998).

"Faccio tutto da solo; mi gestisco io perché i miei lavorano"; "Sto sempre da sola, cucino io. Non sono triste, sono abituata a stare da sola": alcuni frammenti delle interviste descrivono un tempo vissuto in solitudine.

Essi vivono alcune dicotomie importanti che chiedono di essere superate e risolte. Più autonomi, rispetto ai loro coetanei italiani, nella gestione dello spazio e del tempo nel paese d'origine, diventano qui "meno adatti" ad abitare luoghi e ambiti sconosciuti e scarsamente frequentati. Più "grandi" e responsabilizzati qui nella gestione del quotidiano (dal momento che molti di loro si occupano dei loro pasti, della cura della casa, della gestione di fratelli più piccoli. "Mi devo prendere cura di mia nipote perché mia sorella lavora di notte, fa la barista, fino a quando arriva mia mamma") e tuttavia maggiormente dipendenti dalle regole famigliari, dagli obblighi e doveri sanciti dai genitori. Piccoli e grandi; autonomi e dipendenti; responsabilizzati e tenuti al rispetto rigido di obblighi e aspettative: sono alcune delle polarizzazioni significative fra le quali gli adolescenti dell'immigrazione si destreggiano nel quotidiano.

E nel silenzio attraversano anche i momenti di scoraggiamento e di fragilità, reagendo in modo diverso: rifugiandosi nell'idea di bastare a se stessi o fingendo un'allegria che non sentono. Sono soprattutto le ragazze a verbalizzare con sorprendente lucidità i cambiamenti e le emozioni vissuti in solitudine. "Sono quasi sempre triste, quasi sempre. Perché la mia vita ha preso un giro, un giro tremendo che proprio non mi piace. A me però piace sorridere sempre. Anche se sono triste sorrido. A volte mi fa male perché so che sono triste, ma sorrido. Preferisco mostrare che sono (anch'io) felice".

"Adesso sono diventata più fredda e riuscirei anche a vivere da sola. Forse è stato il tempo senza i miei genitori, la lontananza. All'inizio ho sofferto di più, adesso non sento niente". 


\section{Immaginare il futuro}

Sulla cosiddetta "generazione 1.5 " si concentrano spesso aspettative e attese divergenti e di segno opposto. La scuola, gli ambiti di aggregazione, le relazioni con i pari (seppure ridotti nel numero e nell'intensità) propongono modelli di vita, atteggiamenti e scelte indirizzati verso l'adesione al "qui e ora". La famiglia, da parte sua, può aver elaborato aspettative rispetto al presente e al futuro del figlio ricongiunto, che si collocano rigidamente entro i progetti famigliari, gli impegni e le promesse che sempre accompagnano la migrazione. Gli ambiti entro i quali si depositano le diverse attese hanno a che fare con la vita relazionale e i rapporti con i pari, con le scelte scolastiche e lavorative, con i contorni dei progetti e del futuro: riuscire e apprendere a scuola e mantenere intatti fedeltà e legami, adottando comportamenti, e pratiche che si richiamano a valori e riferimenti delle origini. E tuttavia la migrazione modifica i figli in misura profonda e pregnante, ma modifica anche le famiglie, i nuclei, i ruoli. Si vengono così a creare nuove inedite rappresentazioni di quello che si ritiene il "nocciolo duro" dell'appartenenza che è il frutto di perdite, negoziazioni, nuovi confini. Si ridefiniscono così alcuni "oggetti identitari" irrinunciabili (il rispetto dei minori nei confronti degli adulti, è uno di questi) che si vuole/deve trasmettere alla nuova generazione, mentre altri elementi della cosiddetta "cultura d'origine" diventano periferici, meno importanti, modificabili.

A differenza dei più piccoli, nati in Italia e abituati da subito a muoversi e a interagire con due spazi di socializzazione, coloro che migrano nell'adolescenza restano più a lungo "in bilico" tra un lungo passato altrove e un futuro tutto da costruire.

Ma quali sono le attese e i desideri che le ragazze e i ragazzi immigrati elaborano rispetto al loro futuro? La maggior parte immagina il domani qui, in Italia e pensa di ritornare ai luoghi delle origini da turista/viaggiatore. Altri non sanno dove collocare il loro futuro e si mettono nelle mani dei loro genitori: "Non so, vediamo che cosa decidono i miei genitori"; "Metà in Italia e metà in Pakistan": essi affermano, con un po' di rassegnazione e ancora molta provvisorietà. Coloro che hanno vissuto la migrazione come obbligo e costrizione progettano un ritorno dai nonni. Altri ancora prevedono un'ulteriore migrazione, la scelta di un altro paese o città (Londra, la Francia, l'Inghilterra, la Germania) dove ricominciare ancora una volta tutto da capo, ma, questa volta, avendolo scelto la meta del proprio viaggio in prima persona.

La scelta della scuola superiore è fortemente legata al luogo del futuro e rappresenta per ogni ragazzo e ragazza un appuntamento importante e cruciale. Per i minori stranieri, essa assume spesso le caratteristiche proprie di un evento/confine che segna il primo e il dopo; indirizza il presente 
verso un determinato cammino e incanala il futuro nell'una o nell'altra direzione. F. Tawne definisce boundary events gli episodi e le situazioni che segnano il disvelamento di una condizione di minorità e l'ingresso da parte del ragazzo straniero in una fase di dolorosa consapevolezza della propria identità. "Sono episodi che spesso minano la stima di sé e condizionano l'identità di chi sta crescendo e registra con particolare sensibilità il giudizio delle persone che lo circondano" (Granata, 2011).

L'orientamento può essere dunque l'inizio di un processo di marginalizzazione, sociale e lavorativa oltre che scolastica o, viceversa, rappresentare un'opportunità di mobilità e di promozione e inaugurare un percorso di inclusione positiva. È un indicatore indiretto delle possibili opportunità occupazionali e della promozione sociale, dal momento che influenza direttamente i tempi e le modalità di accesso al mercato del lavoro. Per questa ragione, nel tempo delle scelte scolastiche si attivano dispositivi e attenzioni nelle scuole e nelle famiglie; si mobilitano insegnanti e genitori; si suscita il confronto e la composizione fra punti di vista e aspettative differenti.

L'orientamento è un prisma a molte facce. Dal punto di vista temporale, esso convoca e porta sulla scena del presente, da un lato, il passato e la storia scolastica precedente e, dall'altro, la prefigurazione e l'idea di futuro. Dal punto di vista spaziale, esso chiede di collocare le scelte in un determinato luogo e in una data situazione territoriale della quale si intravede il divenire. Dal punto di vista degli attori in gioco, esso riflette le aspettative, le volontà e le promesse degli adulti e dei minori e cerca di coniugare $\mathrm{i}$ vincoli e le risorse, le attese e le capacità individuali con le valutazioni oggettive e le reali possibilità. Soggetti diversi agiscono sulla scena nel momento dell'orientamento e concorrono alle decisioni (i ragazzi, le famiglie, gli insegnanti); tempi e dimensioni differenti s'intrecciano e si compongono: la storia passata dell'alunno, l'adolescenza con le sue sfide e vulnerabilità, i desideri del qui e ora e la prefigurazione del futuro possibile.

Nel caso degli studenti stranieri, immigrati o nati in Italia, questo evento è cruciale per tutti questi aspetti, molti dei quali comuni ai parti autoctoni e lo è anche per altri fattori. Da parte dei minori e delle loro famiglie, si tratta di elaborare decisioni importanti che non sempre sono sostenute da informazioni chiare ed efficaci sulle ragioni delle scelte e di immaginare il futuro senza farsi sovrastare dal vissuto di provvisorietà che permea il presente e pervade il quotidiano. Di far convivere dunque la precarietà dell'essere migrante, resa oggi più acuta dalla situazione attuale di crisi, con la stabilità richiesta da opzioni durature che si collocano nel qui e ora, ma che chiedono di investire sul domani e sul dopodomani. In altre parole, di gestire insieme l'instabilità con la lungimiranza. Ma per poter essere davvero lungimiranti, per poter "guardare avanti e guardare verso l'alto" sono necessarie certamente informazioni, capacità e immaginazione, ma 
servono anche condizioni materiali di relativa tranquillità che possano consentire uno sguardo più largo.

Da parte della scuola e di coloro che si occupano di orientamento, si tratta di proporre suggerimenti e indirizzi che tengano conto di più variabili e che possano comporre $\mathrm{i}$ vincoli e le possibilità dei minori e delle loro famiglie, le aspettative, i progetti e le motivazioni degli uni e degli altri. Ma si tratta anche di proporre consigli orientativi che siano efficaci e non indirizzati da un rappresentazione svalorizzata dell'allievo straniero. Più in generale, le scelte scolastiche dei figli degli immigrati rappresentano un indicatore importante della mobilità sociale ed economica di una determinata società poiché definiscono almeno in parte il "posto" che il minore straniero occuperà domani nel mondo del lavoro e nelle relazioni sociali.

\section{Orientati o disorientati?}

La frequenza/non frequenza della scuola superiore costituiscono dunque ad un tempo uno dei mezzi più potenti di inclusione positiva e di buona integrazione e, viceversa, una delle condizioni che contribuiscono a produrre situazioni di vulnerabilità. Le conclusioni comuni a cui gli studi e le ricerche in proposito pervengono ci dicono che le differenze nel tasso di scolarità nella scuola secondaria di secondo grado tra italiani e stranieri risultano particolarmente elevate: sulla base dei dati ISTAT, esso è infatti del 94\% tra gli italiani e del 63\% tra gli stranieri. Le analisi dei dati mostrano inoltre in maniera evidente che studenti italiani e stranieri non prendono le decisioni sul proseguimento del percorso scolastico dopo la scuola secondaria di primo grado nello stesso modo. I secondi infatti, a parità delle altre condizioni, devono considerare un aspetto in più rispetto ai primi: il fatto di essere stranieri (Canino, 2010). Proprio in conseguenza del fattore "etnico", gli studenti non italiani si trovano nella condizione spesso di "rivedere al ribasso" i propri percorsi formativi. Si delinea dunque una situazione scolastica che presenta tratti di iniquità sociale e che rischia lo spreco di risorse e di talenti, i quali spesso non sono sostenuti e valorizzati in maniera efficace.

I dati nazionali diffusi dal MIUR-ISMU, e soprattutto il capitolo di approfondimento dedicato alla scuola secondaria di secondo grado contenuto nel dossier statistico (MIUR-ISMU, 2011) contengono dunque alcune evidenze e servono a tratteggiare la fisionomia della scuola secondaria di secondo grado multiculturale. In sintesi, emerge che:

- nel momento delle iscrizioni, gli studenti stranieri tendono a fare scelte scolastiche più brevi e meno esigenti che si discostano da quelle degli 
italiani e questo può avvenire anche a prescindere dai risultati scolastici e dal voto ottenuto all'esame di terza media;

- essi scelgono in gran parte i percorsi di studio professionalizzanti e ciò determina una concentrazione delle presenze in particolare nelle prime classi degli istituti professionali e nella formazione professionale;

- il confronto fra le scelte scolastiche dei ragazzi stranieri nati all'estero e quelle dei minori nati in Italia (che sono in numero e in percentuale ancora ridotti) sembra delineare un certo avvicinamento di questi ultimi, rispetto alle modalità di iscrizione dei pari italiani, ma il cambiamento è ancora solo accennato;

- si registrano dati di presenza inferiori nella scuola superiore di studenti provenienti dai contesti africani e asiatici e invece quote e percentuali più consistenti di allievi europei e latinoamericani. Questo dato apre domande e interrogativi sulle situazioni e le ragioni dell'abbandono scolastico da parte di minori appartenenti a determinati Paesi;

- le scelte scolastiche risentono anche delle caratteristiche dei territori e del fattore "attrazione" esercitato dal mondo del lavoro soprattutto in determinati contesti del Nord, Nord-est e del Centro.

\section{Fattori in gioco al momento delle scelte}

Come abbiamo osservato anche fra gli studenti stranieri coinvolti nel progetto "Almeno una stella", quando arriva il momento delle scelte scolastiche, i ragazzi stranieri presentano profili di criticità che indirizzano la prosecuzione degli studi verso percorsi a carattere professionalizzante. La situazione diffusa di ritardo scolastico, l'esperienza della bocciatura incontrata lungo il percorso ed esiti finali non brillanti sono tutti fattori che sono alla base di una canalizzazione formativa di fatto. Ma, oltre alle variabili legate alle carriere scolastiche accidentate, altri fattori possono agire nel momento dell'orientamento. Dall'osservazione di un numero significativo di biografie scolastiche e di casi di orientamento/ri-orientamento osservati nell'ambito del progetto, vediamo che, oltre ai fattori comuni agli italiani, ve ne sono altri che possiamo riferire in particolare alla condizione dei minori stranieri. Le variabili comuni che agiscono nel momento delle scelte scolastiche riguardano soggetti e dimensioni diverse. Li riepiloghiamo brevemente.

Prima fra tutte vi è la dimensione personale che indirizza le scelte sulla base delle preferenze, le aspettative, le attitudini, l'immagine di sé, l'autostima. Un ruolo centrale ha poi la dimensione scolastica, che è definita dal percorso di studi, gli esiti e la qualità dei risultati nelle diverse discipline. Vi sono poi le condizioni e la struttura delle famiglie e, in particolare, il tipo di occupazione del padre, la situazione lavorativa della madre, il reddito famigliare, il capitale culturale e la scolarità dei genitori. Giocano un ruolo 
importante, come abbiamo visto, anche le caratteristiche del territorio, le possibilità occupazionali, le specificità del tessuto produttivo e l'immagine sociale che la scuola e la cultura rivestono in quel contesto. Ma nel caso delle ragazze e dei ragazzi stranieri, entrano in gioco, oltre a questi, anche altri fattori direttamente legati alla condizione di migrazione. Li elenchiamo a partire dai soggetti diversi che agiscono sulla scena nei momenti dell'orientamento: le famiglie, gli insegnanti e i minori.

Oltre alle condizioni socio-economiche e culturali - fattori comuni agli autoctoni - nel caso dei nuclei famigliari immigrati, contribuiscono a indirizzare le scelte di prosecuzione degli studi dei figli anche le caratteristiche del progetto migratorio, le prospettive di stabilità o, viceversa, la provvisorietà del soggiorno; l'atteggiamento verso l'acquisizione della cittadinanza italiana e il posto nel quale la famiglia colloca il suo futuro. Accanto a queste variabili, proprie del vivere e del pensarsi "tra", vi possono essere, da parte dei genitori, anche scarse informazioni sul sistema scolastico italiano e una difficoltà a capire quali sbocchi professionali e lavorativi l'una o l'altra scelta possono aprire, oltre che, talvolta, un'idea differente di futuro e di opportunità professionali sulla base del genere, per i figli e per le figlie.

Per quanto riguarda gli insegnanti e gli operatori, nel momento in cui i elaborano i consigli orientativi, possono essere influenzati inconsapevolmente dalle rappresentazioni che hanno interiorizzato rispetto all'essere "straniero". Quale spazio sociale e professionale essi prefigurano per i nuovi cittadini? Una diffusa concezione "miserabilista" dell'immigrazione, che ne evidenzia solo il carattere di povertà e fragilità sociale e non anche gli elementi di promozione, conquista, opportunità a volte rischia di indirizzare le scelte, come abbiamo visto, verso il basso, verso il "possibile" in condizioni di minorità date per scontate. Un altro fattore che può agire nei confronti degli alunni stranieri e dei percorsi formativi futuri si spiega con una forma di iper-protettività che vorrebbe garantirli da insuccessi scolastici e da frustrazioni. In questa ottica, l'orientamento verso percorsi di studio più brevi e meno esigenti dovrebbe esporre l'allievo non italiano ad un minore rischio di scacco e di esito negativo. E infine, un terzo fattore che entra in gioco nei consigli orientativi ha naturalmente a che fare con la competenza linguistica che gli alunni stranieri hanno raggiunto e che i docenti hanno certificato. In una scuola fortemente verbale, nella quale si insegna e si apprende quasi esclusivamente solo attraverso le lezioni frontali e i testi scritti, la competenza raggiunta nell'italiano dello studio occupa un rilievo centrale. Le difficoltà linguistiche che gli apprendenti non italofoni incontrano nel percorso di apprendimento di una seconda lingua rischiano tuttavia di lasciare nell'ombra gli altri talenti e capacità e di ignorare forme e situazioni di bilinguismo diffuso. Quali tratti specifici, connessi al loro 
essere "stranieri", possiamo infine individuare fra gli allievi non italiani al momento delle decisioni su se e come proseguire gli studi? Naturalmente essi devono fare i conti, come i loro compagni, con gli esiti scolastici, tener conto delle attitudini e delle preferenze di cui pian piano stanno diventando consapevoli, gestire le loro motivazioni, i desideri, la capacità di impegno.

Ma altri "oggetti" e vincoli interni ed esterni fanno sentire il loro peso. In certi casi, le ragazze e i ragazzi stranieri si trovano a prendere da soli delle decisioni per il proprio futuro perché i genitori sono "mediatori deboli", data la loro difficoltà a informarsi, essere informati e gestire le scelte con consapevolezza. In altri casi, i figli devono fare i conti con i progetti, e le aspettative e i vincoli famigliari che possono lasciare poco spazio al desiderio e alla volontà individuali. In tutti i casi, scegliere la scuola superiore vuol dire fare un investimento per un futuro che si deve collocare, oltre che nel tempo di là da venire, anche in uno spazio e in un contesto definitivo.

Scegliere dunque per il "qui" o per l'altrove? Per l'Italia o per il Paese di origine?

Il tempo dell'orientamento s'intreccia così strettamente con il tema della cittadinanza dei minori stranieri perché s'intreccia ed è determinato dal senso di appartenenza/non appartenenza, dal vissuto di chi si sente parte o, viceversa, si sente in disparte rispetto al luogo in cui studia e abita.

\section{Orientarsi: fattori specifici}

Di seguito, alcuni fattori che incidono sulle modalità di orientamento $\mathrm{e}$ sulle scelte scolastiche degli studenti stranieri e che hanno a che fare con la loro condizione di migrazione.

\section{Le famiglie straniere}

- scarse o parziali informazioni sul sistema scolastico italiano e sulla tipologia delle scuole secondarie di secondo grado;

- progetto migratorio ancora indefinito, rispetto al tempo e alla durata (vissuto di provvisorietà che si trasmette ai figli) e rispetto al luogo e al Paese in cui si colloca il futuro della famiglia;

- difficoltà ad accompagnare i figli nel momento delle scelte e a sostenerne motivazioni e progetto.

Gli insegnanti e la scuola

- rappresentazione dell'immigrazione di tipo "miserabilista" e prefigurazione del ruolo sociale e lavorativo dei giovani immigrati più debole e marginale;

- elaborazione di aspettative più basse nei confronti dell'allievo straniero; 
- atteggiamento di iper-protezione che può portare a un consiglio orientativo verso il basso, verso percorsi scolastici più brevi e meno esigenti;

- valutazione non del tutto positiva delle competenze linguistiche in italiano (soprattutto dello studio) che diventa predominante e rischia di lasciare in ombra talenti e capacità.

Gli studenti stranieri

- percorsi scolastici più "accidentati”;

- ritardo scolastico ed età più elevata, rispetto ai compagni, al momento della scelta della scuola superiore;

- solitudine nel momento dell'orientamento e delle decisioni da prendere per la prosecuzione degli studi;

- difficoltà a prefigurare il futuro a partire da vissuti di provvisorietà e di non-cittadinanza.

\section{I "quasi adatti"}

Gli adolescenti stranieri, in seguito all'esperienza migratoria e all'inserimento in un contesto multiculturale, vengono chiamati ad affrontare una serie ulteriore di compiti evolutivi non risolvibili mediante strategie comportamentali precedentemente apprese. Devono imparare a vivere in contesti diversi, adattando flessibilmente il proprio comportamento alle diverse circostanze. Ragazzi "camaleonte" che imparano a cambiare colore, sia per rispondere alle sollecitazioni e alle aspettative differenti, sia per esprimere emozioni e stati d'animo diversi.

Quali strategie di coping adottano i minori immigrati per far fronte alle nuove situazioni di prova e ai compiti di sviluppo aggiuntivi? Il termine coping sta ad indicare la maniera specifica di far fronte a una situazione stressante e di gestirla. Esso può essere definito come un insieme di sforzi cognitivi e affettivi per gestire richieste - interne o esterne all'individuo che sono valutate come eccedenti le risorse della persona. L'utilizzo della parola sforzo vuole sottolineare la percezione dell'individuo di dover fare appello a comportamenti che vanno al di là delle condotte di routine e l'intenzionalità di tale mobilitazione.

"Definito in questo modo il processo di coping, diventa un mediatore tra la percezione dell'individuo e l'ambiente circostante e appare come una dimensione del sé, legata ad attribuzioni causali di tipo interno che permettono al soggetto di percepirsi come capace di influire sulla propria esistenza" (Cesari, 1997). Vi sono minori che adottano preferibilmente strategie orientate verso l'interno, verso se stessi e che sembrano più indirizzati, dal punto di vista delle scelte identitarie, verso il paese di origine. Queste stra- 
tegie, in base alla definizione di Brenner (1984) assumerebbero complessivamente un carattere di negazione del conflitto, come la sottomissione, l'adattamento passivo alla situazione, la rassegnazione, la ricerca di coalizione. Vi sono invece coloro che sono più orientati verso la società di accoglienza e che tendono ad adottare strategie rivolte verso l'esterno: il confronto e lo scontro, una maggiore aggressività, forme di autoaffermazione e di reazione. Cambiando orientamento culturale si è osservato inoltre che i ragazzi immigrati tendono anche a cambiare il tipo di strategie adottate.

Nella sua ricerca sui figli degli immigrati in Germania, A. Portera (1997) ha cercato di approfondire le strategie di coping adottate dai ragazzi intervistati - sia in situazione di riuscita scolastica e buon inserimento sociale, sia in situazione di esclusione e scacco. I casi analizzati tendono a dimostrare che le diverse strategie - verso l'interno o verso l'esterno possono essere di volta in volta le più efficaci in risposta alle diverse situazioni e che non vi è una maggiore adeguatezza di una modalità di risposta sull'altra. Si può aggiungere che in genere, dalla scuola e dai servizi, vengono accolte con maggior favore le modalità di risposta alla situazione stressante che prevedono forme di ripiego e di accettazione, piuttosto che quelle di tipo reattivo e aggressivo. I minori che manifestano attraverso l'impulsività e l'aggressività le difficoltà da loro incontrate nel tentativo di difendere la propria appartenenza culturale e di occupare un posto definito nella famiglia, tendono ad essere stigmatizzati in misura maggiore rispetto a chi si racchiude nel silenzio e nell'apatia.

Oltre a esaminare le diverse strategie, Portera ha individuato dieci fattori positivi e protettivi "che hanno avuto il potere, non solo di diminuire il livello della crisi e di promuoverne il superamento, ma anche, in alcuni casi, hanno persino contribuito a connotare l'emigrazione e l'assunzione di identità in contesto multiculturale come un'esperienza positiva e arricchente". Essi sono:

- le caratteristiche disposizionali o costituzionali di ciascuno;

- il rapporto positivo con almeno una persona di riferimento affettivo durante i primi anni di vita;

- l'apertura dei genitori nei confronti dell'ambiente sociale del paese di immigrazione;

- la comprensione e la fiducia da parte dei genitori;

- la preparazione al distacco e il mantenimento dei legami durante i periodi di separazione;

- l'esperienza positiva di accettazione e di rispetto nel paese di accoglienza soprattutto durante i primi contatti;

- la comprensione da parte degli insegnanti e degli educatori;

- nessuna forzatura verso l'assimilazione a scuola e durante il tempo libero; 
- un ruolo importante giocato dagli amici come funzione ponte tra le due culture

- l'appoggio e il sostegno attraverso i gruppi di doposcuola

- i momenti di ascolto, consulenza o di terapia.

Accogliere i ragazzi immigrati significa accogliere anche le loro storie di viaggio, i cambiamenti che li attraversano, i vissuti di disorientamento rispetto allo spazio, al tempo, alle parole, ai gesti.

Accogliere quindi anche la loro vulnerabilità, potenziale o reale.

La migrazione è per tutti un evento cruciale, da non sottovalutare, da preparare con cura, poiché segna l'avvio di un nuovo capitolo nella storia famigliare e l'inizio del nuovo viaggio di appartenenza che li accoglie. Viaggio da sostenere nelle sue tappe, da facilitare nelle conquiste e da aiutare nelle soste, poiché comporta per i minori che vi sono coinvolti fatiche aggiuntive, ostacoli e sfide da superare. Poiché rappresenta, nelle biografie e nelle storie individuali, una situazione di crisi, ovvero, al tempo stesso, un evento rischioso e un'opportunità da sostenere, da parte dei genitori e di chi accoglie, con attenzione, ascolto consapevole, competenza e cura.

\section{Riferimenti bibliografici}

Beneduce R. (1998), Frontiere dell'identità e della memoria, FrancoAngeli, Milano.

Bertolini P., Lalla M., Toscano V. (2010), L'inserimento scolastico degli studenti stranieri di prima e seconda generazione in Italia, Università degli studi di Modena e Reggio Emilia.

Canino P. (2010), Stranieri si nasce... e si rimane?, Quaderni dell'Osservatorio n. 3 , Fondazione Cariplo.

Ceccagno A. (2004), Giovani migranti cinesi. La seconda generazione a Prato, FrancoAngeli, Milano.

Cesari Lusso V. (1997), Quando la sfida si chiama integrazione. Percorsi di socializzazione e di personalizzazione di giovani figli di emigranti, NIS, Roma.

Colombo E. (a cura di) (2010), Figli di migranti in Italia. Identificazioni, relazioni, pratiche, Utet, Torino.

Dalla Zuanna G., Farina P., Strozza S. (2009), Nuovi italiani. I giovani immigrati cambieranno il nostro paese?, Il Mulino, Bologna.

D’Ignazi P. (2008), Ragazzi immigrati. L'esperienza scolastica degli adolescenti attraverso l'intervista biografica, FrancoAngeli, Milano.

Favaro G., Napoli M. (a cura di) (2002), Come un pesce fuor d'acqua. Il disagio nascosto dei bambini e dei ragazzi immigrati, Guerini, Milano.

Favaro G., Napoli M. (a cura di) (2004), Ragazze e ragazzi nella migrazione. Adolescenti stranieri: identità, racconti, progetti, Guerini, Milano.

Favaro G., Papa N. (2009), Le ragazze e i ragazzi stranieri nella scuola superiore, FrancoAngeli, Milano. 
Giovannini G., Queirolo Palmas L. (a cura di) (2002), Una scuola in comune. Esperienze scolastiche in contesti multietnici italiani, Edizioni della Fondazione Agnelli, Torino.

Granata A. (2011), Sono qui da una vita. Dialogo aperto con le seconde generazioni, Carocci, Roma.

Leonini L. (a cura di) (2004), Stranieri e italiani. Una ricerca tra gli adolescenti figli di immigrati nelle scuole superiori, Donzelli, Roma.

MIUR-ISMU (2011), Alunni con cittadinanza non italiana. Verso l'adolescenza. Rapporto nazionale anno scolastico 2010-2011, www.istruzione.it.

Molina S., Ambrosini M. (a cura di) (2004), Seconde generazioni. Un'introduzione al futuro dell'immigrazione in Italia, Edizioni della Fondazione Agnelli, Torino.

Portera A. (1997), Tesori sommersi. Emigrazione, identità, bisogni educativi interculturali, FrancoAngeli, Milano.

Queirolo Palmas L. (2006), Prove di seconde generazioni. Giovani di origine immigrata tra scuole e spazi urbani, FrancoAngeli, Milano.

Ravecca A. (2009), Studiare nonostante. Capitale sociale e successo scolastico degli studenti di origine immigrata nella scuola superiore, FrancoAngeli, Milano. 


\section{Ritratti e percorsi scolastici dei ragazzi accompagnati}

di Michela Casorati* e Monica Napoli

\section{Le caratteristiche dei ragazzi}

I minori che sono stati accompagnati nel corso delle due annualità del progetto "Almeno una stella" sono stati 461, così suddivisi: 129 a Milano, di cui 56 femmine e 73 maschi; 78 a Torino, di cui 45 femmine e 33 maschi; 98 a Bologna (74 femmine e 24 maschi); 72 ad Arezzo, di cui 34 femmine e 38 maschi; 42 a Trento (16 femmine e 26 maschi); 42 in Friuli, di cui 22 femmine e 20 maschi. I ragazzi sono stati segnalati dalle scuole sulla base dell'esigenza di un rinforzo delle competenze linguistiche o della riuscita scolastica, oltre che di una maggiore socializzazione e integrazione nel gruppo dei pari e nei servizi socio-educativi del territorio.

Tab. 1 - Alunni e studenti accompagnati nei diversi territori

\begin{tabular}{|l|c|c|c|}
\hline Città & Femmine & Maschi & Totale \\
\hline Milano & 56 & 73 & 129 \\
\hline Torino & 45 & 33 & 78 \\
\hline Bologna & 74 & 24 & 98 \\
\hline Arezzo & 34 & 38 & 72 \\
\hline Provincia di Trento & 16 & 26 & 42 \\
\hline Friuli Venezia Giulia & 22 & 20 & 42 \\
\hline Totale & $\mathbf{2 4 7}$ & $\mathbf{2 1 4}$ & $\mathbf{4 6 1}$ \\
\hline
\end{tabular}

* Centro Come - Cooperativa Farsi Prossimo, Milano. 


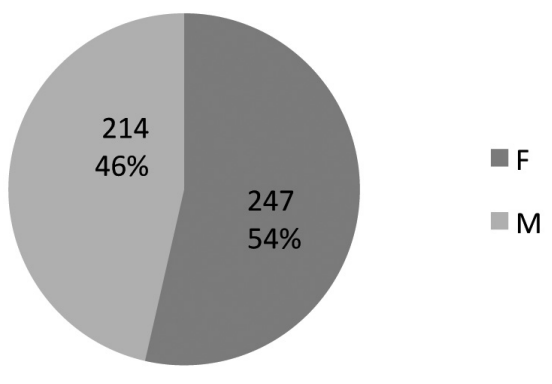

Complessivamente, si è trattato di 247 ragazze (pari al 54\% del totale) e 214 ragazzi (il $46 \%$ del totale), provenienti da 54 differenti Paesi. Le nazionalità più rappresentate sono state: Cina (59 minori), Filippine (55, localizzati soprattutto a Milano); Romania (45 studenti; a Torino e Arezzo il numero maggiore); Marocco (con la maggioranza delle presenze a Bologna e Torino), e Pakistan (soprattutto ragazze, in particolare ad Arezzo e Bologna). A seguire: Moldavia, Perù, Bangladesh (per la maggior parte ragazze), Egitto (quasi tutti maschi), Ecuador, Ucraina, Sri Lanka. A seguire, numerosi altri paesi, ciascuno dei quali rappresentato da numeri molto esigui di destinatari (sotto le cinque unità). Sette erano figli di coppia mista, con un genitore italiano e uno proveniente da un altro Paese.

Tab. 2 - La nazionalità maggiormente rappresentate

\begin{tabular}{|l|c|c|c|}
\hline Paese d'origine & Femmine & Maschi & Totale \\
\hline Cina & 26 & 33 & 59 \\
\hline Filippine & 27 & 28 & 55 \\
\hline Romania & 21 & 24 & 45 \\
\hline Marocco & 26 & 19 & 45 \\
\hline Pakistan & 24 & 10 & 34 \\
\hline Moldavia & 14 & 9 & 23 \\
\hline Perù & 14 & 9 & 23 \\
\hline Bangladesh & 16 & 5 & 21 \\
\hline Egitto & 2 & 13 & 15 \\
\hline Ecuador & 7 & 8 & 15 \\
\hline Ucraina & 8 & 4 & 12 \\
\hline Sri Lanka & 5 & 5 & 10 \\
\hline Altri paesi & 57 & 47 & 104 \\
\hline Totale & $\mathbf{2 4 7}$ & $\mathbf{2 1 4}$ & $\mathbf{4 6 1}$ \\
\hline
\end{tabular}


Le provenienze dei ragazzi corrispondono alla distribuzione nazionale sui diversi territori: le nazionalità maggiormente presenti nelle diverse città sono indicate nella tabella seguente (Tab. 3). Il numero tra parentesi indica i minori raggiunti sul territorio appartenenti a ciascuna nazionalità. A Milano, Cina e Filippine raccolgono la maggior parte delle presenze; sono rappresentati molti altri Paesi, ma con numeri più contenuti. A Torino, prevalgono i minori marocchini e le altre nazionalità, pur numerose, hanno numeri decisamente inferiori. Ad Arezzo, le prime tre nazionalità risultano bilanciate e con presenze più significative rispetto agli altri Paesi. A Bologna, le provenienze sono molto variegate; le principali nazionalità (Moldavia, Pakistan, Cina) hanno numeri simili. Nelle scuole trentine, si registra un numero estremamente ampio di provenienze. I ragazzi maggiormente seguiti sono di nazionalità cinese; al secondo posto, a parità di presenze, si collocano macedoni, marocchini e pakistani, seguiti subito dopo dai rumeni. Infine, in Friuli, più significativa è la presenza di alunni bengalesi, seguiti dai rumeni, che staccano in modo significativo le altre nazionalità guidate dai ragazzi di origine ucraina.

Tab. 3 - Maggiori presenze nei diversi territori

\begin{tabular}{|l|l|l|l|}
\hline Territorio & Prima presenza & Seconda presenza & Terza presenza \\
\hline Milano & Cina (30) & Filippine (29) & Ecuador (11) \\
\hline Torino & Marocco (24) & Romania (14) & Egitto (5) \\
\hline Bologna & Moldavia (14) & $\begin{array}{l}\text { Cina (11) } \\
\text { e Pakistan (11) }\end{array}$ & $\begin{array}{l}\text { Marocco (10) } \\
\text { e Filippine (10) }\end{array}$ \\
\hline Arezzo & Pakistan (16) & Filippine (13) & Romania (12) \\
\hline Provincia di Trento & Cina (7) & Macedonia (6) & Marocco (6) \\
\hline Friuli Venezia Giulia & Bangladesh (8) & Romania (6) & Ucraina (4) \\
\hline
\end{tabular}

\section{Le situazioni scolastiche e linguistiche}

Com'era al momento dell'ingresso nel progetto la situazione scolastica dei ragazzi seguiti? Purtroppo, come emerge dai dati nazionali, per chi fa più fatica si evidenzia una situazione di forte ritardo rispetto all'età anagrafica. Sulla totalità degli alunni incontrati, solo 161 sono stati inseriti nella classe corrispondente all'età anagrafica: 30 a Milano (poco più del 23\% dei ragazzi inseriti); 30 a Torino (poco meno del 40\%); 24 in Friuli; 23 ad Arezzo (il 32\%); 10 a Trento (il 24\%) e 44 a Bologna (circa il 50\%). Il dato 
più positivo si ritrova quindi in Friuli Venezia Giulia: su 42 ragazzi seguiti, quasi il $60 \%$ degli alunni non evidenzia situazioni di ritardo.

Dei restanti: 178 sono stati inseriti con un anno di ritardo; 93 con due anni; 26 con tre o più anni. Una ragazza della Moldavia non promossa in una secondaria di secondo grado di Torino aveva addirittura 6 anni di differenza rispetto ai compagni di classe.

Il ritardo riguarda in misura simile ragazze e ragazzi, anche nelle situazioni di maggior distanza: su 297 alunni e studenti con situazione di ritardo, 153 sono ragazze e 144 maschi.

\section{Graf. 2 - Ritardo scolastico e anni di ritardo}

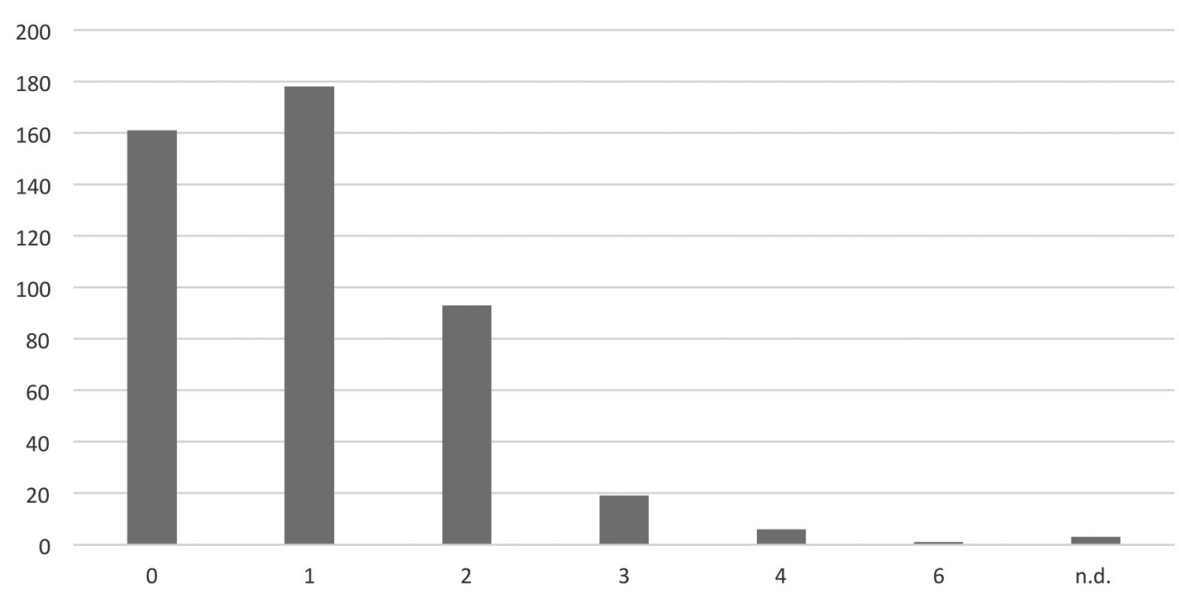

Per quanto riguarda invece il livello linguistico, disponiamo dei dati relativi a 403 studenti. Di questi, solo 10 erano principianti assoluti; 74 erano di livello iniziale; 14 si collocavano tra A1 e A2; 121 di livello A2; 17 tra l'A2 e il B1; 76 B1; 3 tra B1 e B2, 62 di livello B2, 23 (torinesi e bolognesi) di livello $\mathrm{C} 1$ e $\mathrm{C} 2$. Questo conferma la necessità di attivare interventi non solo per chi è appena arrivato, ma anche per chi, già inserito in ritardo, necessita di un aiuto nel superare l'iniziale livello comunicativo e nell'ottenere risultati significativi anche nel rendimento scolastico. 


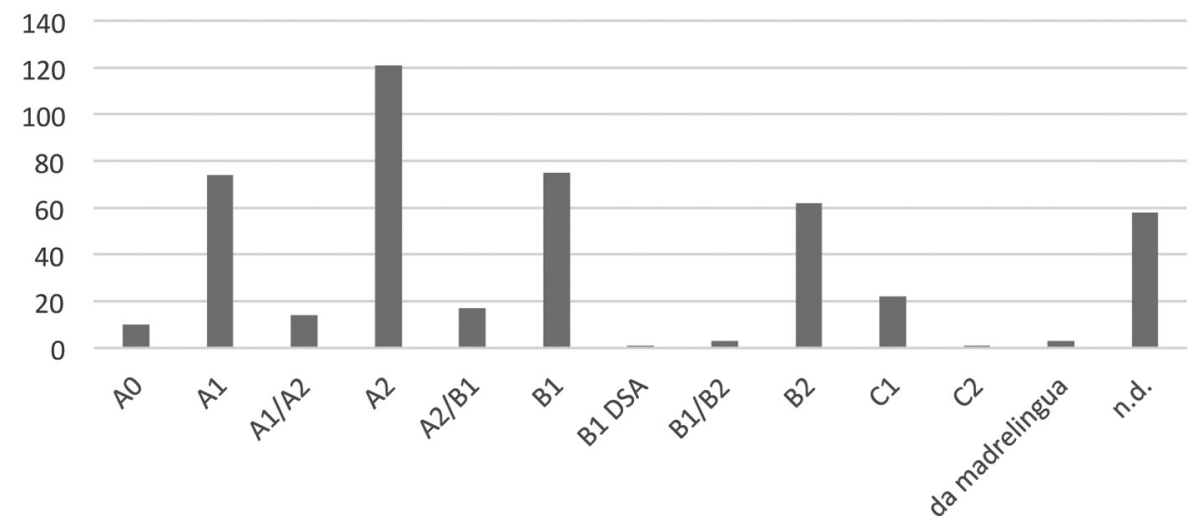

\section{Gli interventi realizzati nel primo anno}

A fronte delle caratteristiche dei ragazzi selezionati, nel corso del primo anno di progetto sono stati attivati interventi specifici e mirati; diversi, come si diceva, a seconda delle esigenze territoriali (cap. 2).

A Milano, l'attività si è concentrata nella definizione e gestione di laboratori per la preparazione degli esami di terza media, per aiutare gli alunni in particolare neoarrivati, a sostenere le prove scritte ed orali. A Torino, è stato strutturato un percorso extrascolastico rivolto agli studenti della prima classe della scuola secondaria di II grado, con l'obiettivo di sostenerli nelle studio delle materie specifiche e dell'italiano L2. Inoltre sono state realizzate diverse attività di accompagnamento e conoscenza del territorio. A Bologna, l'azione di sostegno linguistico-didattico e di tutoraggio si è concentrata specificamente nel biennio della scuola superiore. Ad Arezzo, l'attenzione è stata posta sulla preparazione all'esame di terza media al fine di dare un supporto specifico e intensivo allo studio mediante il potenziamento delle abilità scritte e orali. A Trento, i percorsi, avviati in contesto extrascolastico, si sono focalizzati sugli aspetti relazionali e di esplorazione delle opportunità presenti nel territorio, offrendo anche ai ragazzi occasioni preziose per interagire in lingua italiana e supportandoli nella preparazione del colloquio orale dell'esame di stato. A Udine, Pordenone, Gorizia e relative province, le attività si sono concentrate sull'accompagnamento dal primo al secondo ciclo di istruzione per gli studenti neoarrivati e sull'orientamento al sistema scolastico per studenti e famiglie. 
Dei 461 studenti coinvolti complessivamente, 291 hanno preso parte al progetto nel primo anno. Essi sono stati segnalati dalle scuole e individuati in particolar modo fra coloro che erano giunti in Italia da poco tempo (i cosiddetti NAI), avevano difficoltà e bisogni linguistici legati all'apprendimento dell'italiano L2 e necessitavano di un aiuto in tempo extrascolastico. Si trattava quindi non degli alunni stranieri "medi", ma di coloro che in questa fase del loro percorso scolastico necessitavano di attenzioni, supporto e forme di facilitazione mirata a causa della loro condizione di vulnerabilità, connessa alla migrazione recente, alla non italofonia, alla situazione di solitudine vissuta al di fuori dalla scuola.

Tab. 4 - Studenti coinvolti nel primo anno, per città

\begin{tabular}{|l|c|}
\hline Città & Numero \\
\hline Arezzo & 57 \\
\hline Bologna & 62 \\
\hline Milano & 62 \\
\hline Torino & 60 \\
\hline Trento & 26 \\
\hline Friuli Venezia Giulia & 24 \\
\hline Totale & $\mathbf{2 9 1}$ \\
\hline
\end{tabular}

I ragazzi erano inseriti in prevalenza nelle scuole secondarie di secondo grado (154, il 53\% del totale del primo anno), soprattutto negli istituti professionali e, in misura di poco inferiore (137, il 47\%), nell'ultimo anno della scuola secondaria di primo grado.

Graf. 4 - Distribuzione per ordine di scuola nel primo anno di progetto

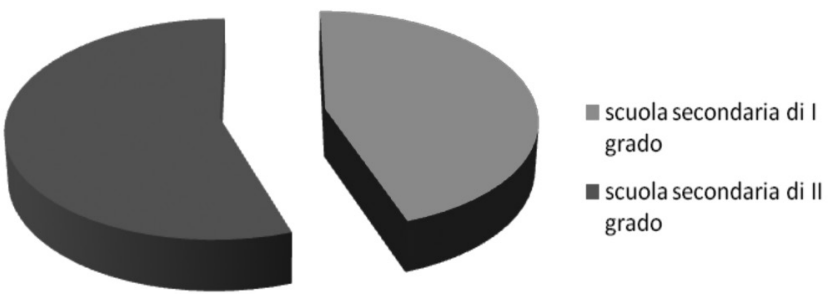



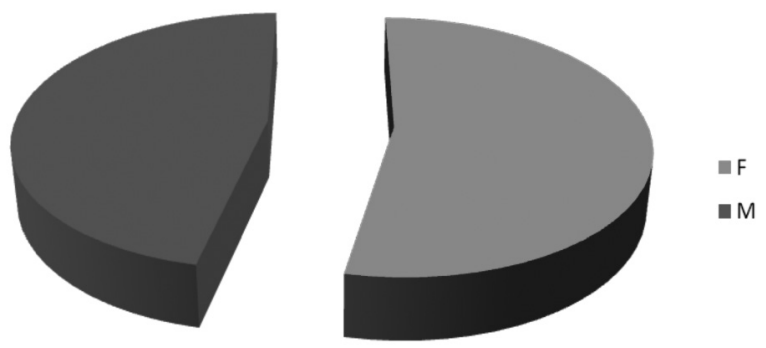

Gli adolescenti seguiti provenivano da diversi Paesi, ma già nel primo anno le prime cinque nazionalità rappresentate (Cina, Marocco, Romania, Filippine, Pakistan) raggruppavano da sole più della metà dei destinatari. L'eterogeneità delle provenienze rappresenta quindi la norma in tutti i territori, con alcune specificità locali.

Graf. 6 - La distribuzione per nazionalità nel primo anno. Paesi più rappresentati

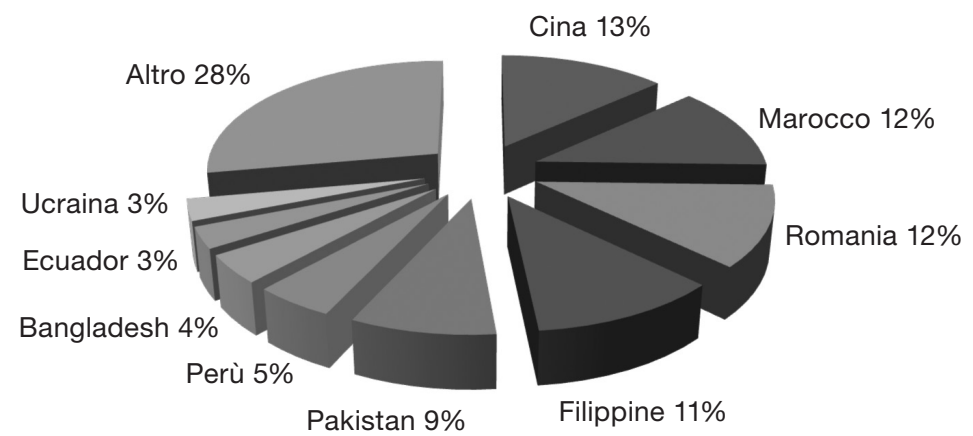

\section{Gli esiti alla fine del primo anno}

Dei 291 ragazzi accompagnati nella prima annualità di progetto, 269 hanno partecipato alle attività in maniera continuativa; 12 hanno interrotto la frequenza per motivi diversi (problemi familiari, ritorno nel Paese d'origine, impossibilità a frequentare nei giorni individuati...); 3 si sono ritirati da scuola nel corso del progetto; di 7 (6 aretini e 1 di Udine) non è più stato possibile recuperare il dato dopo l'uscita dalle attività. 
Dei 269, 124 (pari al 46,1\%), sono stati promossi o ammessi alla classe successiva; 59 (il 21,9\%), non sono stati ammessi; 85 (il 31,6\%), sono stati rimandati dopo il primo anno di superiore e hanno dovuto sostenere gli esami di riparazione a settembre. I dati evidenziano un sostanziale equilibrio rispetto agli esiti tra maschi e femmine, ma un dato più marcato di interruzioni di frequenza alle attività nei ragazzi.

Tab. 5 - Esiti scolastici alla fine del primo anno

\begin{tabular}{|l|c|c|c|}
\hline Esiti scolastici & Femmine & Maschi & Totale \\
\hline Promosso/Ammesso alla classe successiva & 63 & 62 & 124 \\
\hline Giudizio sospeso & 54 & 31 & 85 \\
\hline Non ammesso & 31 & 28 & 59 \\
\hline Bocciato & 0 & 1 & 1 \\
\hline Interruzione della frequenza & 3 & 9 & 12 \\
\hline Ritirato & 1 & 2 & 3 \\
\hline N.d. & 3 & 4 & 7 \\
\hline Totale & $\mathbf{1 5 5}$ & $\mathbf{1 3 6}$ & $\mathbf{2 9 1}$ \\
\hline
\end{tabular}

Graf. 7 - Esiti scolastici alla fine del primo anno

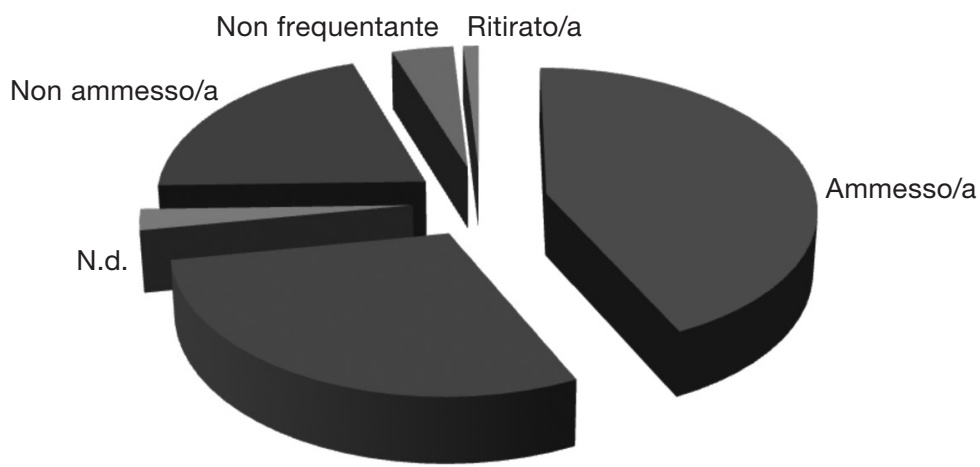

Giudizio sospeso

Se osserviamo gli esiti distinguendo tra gli ordini di scuole, i risultati cambiano in maniera decisa. Nella scuola secondaria di I grado infatti, sui 110 alunni che hanno partecipato ai laboratori in maniera continuativa, 89 (' $81 \%$ ) sono stati promossi e 20 (il 18\%) non sono stati ammessi all'esame di Stato. Solo uno è stato ammesso e poi bocciato all'esame.

Nella scuola secondaria di II grado invece, gli esiti sono stati negativi o sono rimasti sospesi in maniera più diffusa e preoccupante. Su 159 stu- 
denti, solo 35 sono stati promossi (il 22\%); 39 sono stati respinti (il 24,5\%) e ben 85 , pari al 53,5\% hanno atteso gli esami di riparazione di settembre per il giudizio definitivo.

Tab. 6 - Esiti del primo anno suddivisi per tipologia di scuola

\begin{tabular}{|l|c|c|c|}
\hline \multicolumn{5}{|c|}{ Scuola secondaria di I grado } \\
\hline & Femmine & Maschi & Totale \\
\hline Ammessi e promossi & 39 & 50 & 89 \\
\hline Ammessi e bocciati & 0 & 1 & 1 \\
\hline Non ammessi all'esame & 9 & 11 & 20 \\
\hline Totale & $\mathbf{4 8}$ & $\mathbf{6 2}$ & $\mathbf{1 1 0}$ \\
\hline \multicolumn{2}{|c|}{ Scuola secondaria di II grado } & 35 \\
\hline Promossi & 24 & 11 & 39 \\
\hline Bocciati & 22 & 17 & 85 \\
\hline Rimandati a settembre & 54 & 31 & $\mathbf{1 5 9}$ \\
\hline Totale & $\mathbf{1 0 0}$ & $\mathbf{5 9}$ & \\
\hline
\end{tabular}

Questo da un lato sottolinea l'efficacia degli interventi a sostegno dell'esame di terza media. Le prove d'esame, seppur complesse, rappresentano un obiettivo molto preciso e specifico da raggiungere, per il quale i ragazzi vengono gradualmente accompagnati dai docenti nel corso dell'anno, e rispetto al quale anche gli operatori in affiancamento e i tutor possono intervenire in modo mirato. Le finalità sono chiare e il rinforzo avviene in particolare sulle competenze di produzione scritta e orale, sulle principali forme grammaticali, sul lessico specifico. Si aiutano gli alunni ad acquisire un metodo di studio finalizzato a selezionare le informazioni utili allo studio e all'esposizione dei contenuti d'esame; si lavora sulla produzione della tesina da presentare all'orale. Il compito degli operatori e dei tutor è chiaro e definito, e ben delimitato rispetto a quello dei docenti di classe. Il laboratorio è distribuito lungo tutto il secondo quadrimestre, per un tempo sufficientemente lungo e con un articolazione tale da dosare correttamente risorse e forze in vista della prova finale. I buoni esiti ne dimostrano l'efficacia anche su situazioni iniziali di fragilità.

Se andiamo ad analizzare la relazione tra voto di uscita dall'esame e scelte scolastiche nelle superiori, vediamo però che, nonostante i buoni esiti e l'accompagnamento progettuale, le scelte successive sono spesso "canalizzate". Il liceo (per lo più linguistico) viene scelto da pochissimi, e paradossalmente, in proporzione, da quelli con i voti più bassi. Le maggio- 
ri preferenze, indipendentemente dal voto, vanno agli istituti professionali (segreteria e amministrazione, ristorazione e settore alberghiero, servizi socio-sanitari, moda...) e tecnici (soprattutto turistici, commerciali, alberghieri, amministrativi). La formazione professionale (corsi per elettricista, meccanico, parrucchiere, estetista, panificatore...) viene invece scelta da chi ha conseguito il voto più basso, e soprattutto dai ragazzi. Bisogna comunque tener conto del fatto che solo nelle città più grandi è possibile avere a disposizione un'ampia varietà di istituti, mentre nei comuni più piccoli la scelta può essere condizionata dalla vicinanza della scuola a casa e dalla sua raggiungibilità.

Tab. 7 - Voti conseguiti all'esame di terza media e scelte scolastiche

\begin{tabular}{|c|c|c|c|}
\hline Voto conclusivo & Femmine & Maschi & Totale \\
\hline Sei & 18 & 41 & 59 \\
\hline Formazione professionale & 4 & 8 & 12 \\
\hline Istituto professionale & 8 & 10 & 18 \\
\hline Istituto tecnico & 2 & 13 & 15 \\
\hline Liceo & 3 & 2 & 5 \\
\hline Ritorno al Paese d'Origine & 0 & 2 & 2 \\
\hline N.d. & 1 & 6 & 7 \\
\hline Sette & 12 & 7 & 19 \\
\hline CTP & 1 & 0 & 1 \\
\hline Formazione professionale & 1 & 1 & 2 \\
\hline Istituto professionale & 4 & 2 & 6 \\
\hline Istituto tecnico & 6 & 3 & 9 \\
\hline Liceo & 0 & 1 & 1 \\
\hline Otto & 7 & 1 & 8 \\
\hline Istituto professionale & 0 & 1 & 1 \\
\hline Istituto tecnico & 6 & 0 & 6 \\
\hline Liceo & 1 & 0 & 1 \\
\hline Nove & 0 & 0 & 0 \\
\hline Dieci & 2 & 0 & 2 \\
\hline Istituto professionale & 2 & 0 & 2 \\
\hline N.d. & 0 & 1 & 1 \\
\hline Formazione professionale & 0 & 1 & 1 \\
\hline Totale & 39 & 50 & 89 \\
\hline
\end{tabular}


Per quanto riguarda invece gli esiti relativi agli interventi nel primo anno della superiore, le fragilità sottolineate evidenziano la maggior complessità nel proporre azioni mirate a sostegno dello studio. L'accompagnamento extrascolastico è efficace e significativo nel momento in cui opera sul rinforzo di competenze trasversali e di base, utili a tutto il percorso scolastico. Spesso però viene vissuto dai ragazzi e dagli stessi docenti come l'occasione per facilitare l'esecuzione dei compiti per la lezione successiva. Sicuramente un aiuto immediato, ma troppo contingente. Un intervento di questo tipo è invece utile se consente allo studente di estrapolare dall'unità specifica informazioni e contenuti (strutture linguistiche e lessicali, vocaboli della microlingua) più ampi e generali, e gli permette di riflettere su di essi. Il passaggio alla scuola secondaria di secondo grado spesso mette gli studenti di fronte ad un modello diverso di studio e apprendimento rispetto a quello cui erano abituati; è richiesta una maggiore autonomia e capacità di memorizzazione ed elaborazione dei contenuti. Le materie sono nuove, e le microlingue specialistiche. Le attività ad integrazione si trovano perciò a dover sostenere sfide ben più ampie e complesse, con un mandato più vago e generico da parte dei docenti referenti, e obiettivi ben più complessi da raggiungere da parte dei ragazzi. Sottolineiamo però che, nonostante la complessità, questo tipo di intervento è fondamentale per gli studenti, spesso soli di fronte a questa nuova sfida, con livelli di lingua insufficienti e una famiglia non in grado di sostenerli nello studio. Gli esiti positivi degli esami di riparazione lo testimoniano: degli 85 ragazzi rimandati, siamo riusciti a ricostruire gli esiti di 77 studenti; di 8 (tutti trentini) non è stato possibile recuperare il dato. Ci domandiamo quindi come sarebbero stati i risultati finali di questi ragazzi in assenza dell'intervento di "Almeno una stella".

Tab. 8 - Esiti degli esami di riparazione a settembre

\begin{tabular}{|l|c|c|c|}
\hline \multicolumn{4}{|c|}{ Esito finale esami di riparazione dopo il I anno di scuola superiore } \\
\hline & Femmine & Maschi & Totale \\
\hline Ammessi alla II classe & 36 & 21 & 57 \\
\hline Bocciati & 11 & 9 & 20 \\
\hline N.d. & 7 & 1 & 8 \\
\hline Totale & $\mathbf{4 7}$ & $\mathbf{3 0}$ & $\mathbf{8 5}$ \\
\hline
\end{tabular}

Se proviamo a ricostruire complessivamente la situazione alla fine del primo anno scolastico di progetto e proviamo a leggere gli esiti complessivi dopo gli esami di recupero, la situazione è la seguente: 
Tab. 9 - Esiti definitivi alla fine del primo anno dopo gli esami di riparazione

\begin{tabular}{|l|c|c|c|}
\hline Esiti scolastici & Femmine & Maschi & Totale \\
\hline Promosso/ammesso alla classe successiva & 63 & 61 & 124 \\
\hline Non ammesso/bocciato & 31 & 29 & 60 \\
\hline Giudizio sospeso e poi ammissione alla II & 36 & 21 & 57 \\
\hline Giudizio sospeso e poi bocciatura & 11 & 9 & 20 \\
\hline Interruzione di frequenza & 3 & 9 & 12 \\
\hline Ritirato & 1 & 2 & 3 \\
\hline N.d. & 10 & 5 & 15 \\
\hline Totale & $\mathbf{1 5 5}$ & $\mathbf{1 3 6}$ & $\mathbf{2 9 1}$ \\
\hline
\end{tabular}

Complessivamente, gli esiti risultano quindi i seguenti: sono stati promossi 181 ragazzi (il 62,2\% di tutti i ragazzi seguiti nell'attività). Se consideriamo però alla fine del primo anno quelli effettivamente monitorati (261, tolte le interruzioni di frequenza, i ritiri e i non disponibili), la percentuale si alza al 69,3\%. Il dato negativo (bocciatura a giugno o dopo gli esami di riparazione) riguarda invece il $27,5 \%$ circa degli studenti (il $30,6 \%$ circa dei frequentanti). Non si evidenziano differenze particolari tra maschi e femmine, se non una maggiore ricorsività di ritiri e interruzioni di frequenza tra i maschi.

Tab. 10 - Sintesi complessiva fine prima annualità

\begin{tabular}{|l|r|r|c|}
\hline Esiti scolastici & Femmine & Maschi & Totale \\
\hline Promosso/ammesso alla classe successiva & 99 & 82 & 181 \\
\hline Non ammesso/bocciato & 42 & 38 & 80 \\
\hline Interruzione di frequenza/ritiri & 4 & 11 & 15 \\
\hline N.d. & 10 & 5 & 15 \\
\hline Totale & $\mathbf{1 5 5}$ & $\mathbf{1 3 6}$ & $\mathbf{2 9 1}$ \\
\hline
\end{tabular}

\section{Gli interventi realizzati nel secondo anno}

A partire dagli esiti evidenziati a conclusione della prima annualità, per il secondo anno di progetto sono stati attivati interventi e laboratori che coinvolgessero il numero più alto possibile di ragazzi già accompagnati l'anno precedente. 
A Milano si è cercato di seguire il più possibile gli studenti usciti dalle medie nel loro inserimento nella scuola superiore, all'interno degli istituti secondari da loro scelti. Vista la forte distribuzione dei ragazzi in numerose scuole della città, con poche presenze per ciascun istituto, si è comunque valutato di estendere l'offerta dei laboratori anche ad altri studenti neoarrivati, individuati dai docenti delle scuole secondarie di secondo grado.

Anche a Torino si è continuato il tutoraggio degli studenti già coinvolti nella prima annualità: sia quelli promossi e inseriti in seconda, sia quelli bocciati che ripetevano il primo anno. Anche in questo contesto ai ragazzi già seguiti si sono aggiunti nuovi studenti.

A Bologna è proseguita l'azione di sostegno linguistico-didattico e di tutoraggio nelle scuole superiori già seguite nel primo anno.

Ad Arezzo e in Friuli, concluso l'intervento nelle medie, si è focalizzata l'attenzione sul biennio della scuola secondaria di secondo grado.

A Trento, l'intervento è continuato sia nella scuola secondaria di primo grado che nella secondaria.

La situazione di partenza delle azioni del secondo anno ha visto quindi, oltre ai ragazzi già seguiti nella prima annualità di progetto (promossi o bocciati), l'inserimento di nuovi studenti nei laboratori attivati nelle scuole superiori dei diversi territori o nelle sedi degli enti partner. Ai 291 studenti seguiti nel primo anno si sono aggiunti quindi altri 170 studenti, che hanno potuto avvalersi anch'essi delle risorse messe a disposizione del progetto. Come si diceva inizialmente, per tutto l'arco temporale di durata di "Almeno una stella" sono stati intercettati ben 461 studenti. Alcuni hanno proseguito l'intervento nel secondo anno; altri non hanno più frequentato $\mathrm{i}$ laboratori, per motivi diversi: esigenze personali (eccessivo carico di lavoro scolastico, orari incompatibili, trasferimento, rientro nel Paese d'origine...), ma anche obiettivi educativi didattici raggiunti, per cui si è valutato di non proseguire con l'accompagnamento.

Abbiamo comunque cercato il più possibile di mantenere un contatto anche con chi non era più inserito nelle attività, per capire come procedeva la loro carriera scolastica e il loro cammino di integrazione.

Approfondiamo allora le caratteristiche dei ragazzi seguiti nel secondo anno: i "vecchi", i nuovi, quelli inseriti nelle attività e quelli non più seguiti.

In apertura di capitolo abbiamo visto il loro genere e i Paesi di provenienza. Vediamo ora invece la loro distribuzione scolastica nel secondo anno di progetto: 199 erano inseriti in istituti tecnici (in particolar modo ad indirizzo informatico, amministrativo-economico, turistico); 79 in istituti professionali ad indirizzo alberghiero, socio sanitario, meccanico, ecc.; 69 erano iscritti al liceo (scientifico, artistico e linguistico per la maggior parte). 22 studenti hanno scelto corsi di formazione professionale di durata più 
breve, nei settori ristorazione, cura della persona, elettricista, parrucchiere, ecc.

17 (sui 20 che erano stati bocciati l'anno precedente; degli altri 3 non siamo riusciti a ricostruire il dato) hanno ripetuto l'ultima classe della scuola secondaria di primo grado; altri 11 ragazzi inseriti in terza sono stati seguiti per la prima volta nel corso della seconda annualità sul territorio di Trento. In numero minore risultano invece gli studenti:

- che si sono trasferiti in altra città o in un altro Paese (2 ragazzi marocchini che abitavano a Torino sono andati ad abitare in Francia con le loro famiglie);

- che hanno abbandonato gli studi (solo 2, a Torino: un ragazzo iraniano che dopo la bocciatura alle superiori è andato a lavorare in un'officina meccanica, e una ragazza peruviana, anche lei bocciata);

- che sono tornati al Paese di origine (4 ragazze - due aretine, rientrate in Romania, una milanese rientrata in Bangladesh e una torinese rientrata in Moldavia - e 3 maschi - due milanesi rientrati in Perù e nelle Filippine, e un ragazzo di Pordenone rimpatriato in Burkina Faso).

Di 39, tra cui ben 24 maschi, non è stato possibile recuperare il dato.

Tab. 11 - Tipologia di scuola frequentata nella seconda annualità di progetto

\begin{tabular}{|l|r|r|r|}
\hline Tipologia di scuola & F & M & Totale \\
\hline Istituto Tecnico & 113 & 86 & 199 \\
\hline Istituto Professionale & 40 & 39 & 79 \\
\hline Liceo & 46 & 23 & 69 \\
\hline Centri di Formazione Professionale & 10 & 12 & 22 \\
\hline Ripetizione III classe scuola secondaria di primo grado & 8 & 9 & 17 \\
\hline III classe scuola secondaria di primo grado (Trento) & 5 & 6 & 11 \\
\hline Secondaria di secondo grado non specificata & 3 & 7 & 10 \\
\hline Ritorno al paese di origine & 4 & 3 & 7 \\
\hline Trasferito & 1 & 4 & 5 \\
\hline Abbandono scolastico & 1 & 1 & 2 \\
\hline CTP & 1 & 0 & 1 \\
\hline N.d. & 15 & 24 & 39 \\
\hline Totale complessivo & $\mathbf{2 4 7}$ & $\mathbf{2 1 4}$ & $\mathbf{4 6 1}$ \\
\hline
\end{tabular}

Se analizziamo le differenze di genere, le ragazze sembrano scegliere in misura lievemente superiore gli studi più impegnativi (licei e tecnici); fra $\mathrm{i} 79$ inseriti negli istituti professionali in proporzione sono molto più 
numerosi i maschi (39). I dati incoraggianti che emergono dal monitoraggio degli studenti seguiti nel progetto deve però tener conto del fatto che le attività realizzate erano spesso inserite nelle scuole del territorio, tecniche e professionali: hanno quindi intercettato fin da subito e principalmente $\mathrm{i}$ destinatari delle scuole stesse.

Se leggiamo infatti la distribuzione scolastica di coloro che hanno frequentato attivamente i laboratori del secondo anno (258 ragazzi, 153 femmine e 105 maschi), la maggioranza è inserita negli istituti tecnici, e a seguire nei licei e nei professionali dei diversi territori.

Graf. 8 - Distribuzione di genere tra i frequentanti il secondo anno

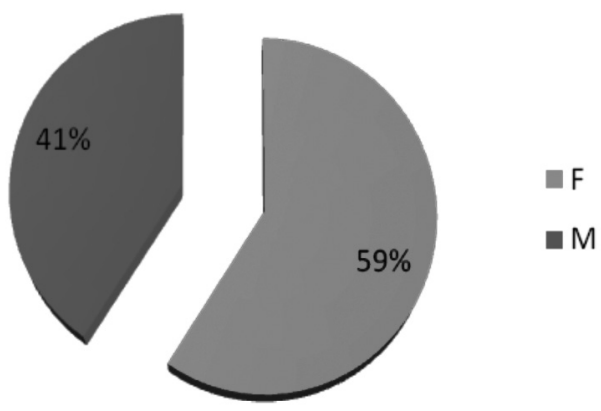

Tab. 12 - Distribuzione scolastica dei frequentanti le attività del secondo anno

\begin{tabular}{|l|r|r|c|}
\hline Tipologia di scuola & F & M & Totale \\
\hline Istituto Tecnico & 86 & 66 & 152 \\
\hline Liceo & 31 & 12 & 43 \\
\hline Istituto Professionale & 25 & 16 & 41 \\
\hline III classe scuola secondaria di primo grado (Trento) & 5 & 5 & 10 \\
\hline Centri di Formazione Professionale & 3 & 4 & 7 \\
\hline Secondaria di secondo grado non specificata & 3 & 2 & 5 \\
\hline Totale complessivo & $\mathbf{1 5 3}$ & $\mathbf{1 0 5}$ & $\mathbf{2 5 8}$ \\
\hline
\end{tabular}

La distribuzione territoriale vede un mantenimento dei numeri già intercettati nel primo anno a Milano e Torino, un forte incremento di partecipanti a Bologna, una riduzione della metà dei frequentanti a Trento e in Friuli. La riduzione più consistente si registra ad Arezzo, che, a causa della dispersione scolastica territoriale e della difficoltà a inserire il progetto 
nella programmazione scolastica delle superiori del territorio, è riuscita a raggiungere nel secondo anno meno di un terzo dei destinatari coinvolti nel primo anno.

Tab. 13 - Studenti frequentanti i laboratori nel secondo anno per città aderente

\begin{tabular}{|l|c|c|c|}
\hline Città & Femmine & Maschi & Totale \\
\hline Arezzo & 11 & 5 & 16 \\
\hline Bologna & 66 & 23 & 89 \\
\hline Milano & 29 & 39 & 68 \\
\hline Torino & 36 & 25 & 61 \\
\hline Trento & 5 & 5 & 10 \\
\hline Udine e territori afferenti & 6 & 8 & 14 \\
\hline Totale & $\mathbf{1 5 3}$ & $\mathbf{1 0 5}$ & $\mathbf{2 5 8}$ \\
\hline
\end{tabular}

\section{Gli esiti alla fine del secondo anno}

Quali sono i risultati dei ragazzi accompagnati nel secondo anno? Ad una prima valutazione, si può notare come anche il secondo anno dimostri l'efficacia dei laboratori per la terza media: sono stati infatti promossi tutti e 10 gli alunni trentini seguiti. Per quanto riguarda la secondaria di secondo grado, invece, su 248 studenti inseriti in scuole di ordini diversi, è stato promosso il 39,5\% dei ragazzi seguiti, e sospeso in attesa degli esami di settembre circa il $34 \%$. Il progetto si è concluso in estate; in questo testo non è stato quindi possibile monitorare i risultati definitivi di chi ha sostenuto la prova di riparazione. Complessivamente però, abbiamo un dato temporaneo non negativo per circa il $75 \%$ dei ragazzi seguiti. Ricordiamo infatti che alla fine degli esami di riparazione relativi alla prima annualità, su 77 studenti ne erano stati promossi 57 (il 67\%). Immaginiamo quindi che la percentuale positiva possa essere stata significativamente più alta dopo le prove di settembre. In questo secondo anno emerge inoltre in modo significativo la differenza di genere: le ragazze alle superiori hanno esiti ben più positivi dei compagni maschi. Il risultato negativo riguarda il $22,5 \%$ degli studenti, mentre non è stato possibile conoscere l'esito di ben 9 ragazzi seguiti, in particolare maschi. Rispetto al rapporto tra tipologia di scuola ed esiti, notiamo come il liceo registri il numero più alto di insuccessi (10 bocciature su 25 frequentanti), mentre al tecnico i promossi sono quasi il doppio dei bocciati. 
Tab. 14 - Esiti scolastici alunni frequentanti la seconda annualità

\begin{tabular}{|c|c|c|c|}
\hline Esiti scolastici & $\mathbf{F}$ & M & Totale \\
\hline Promossi in terza media (Trento), di cui: & 5 & 5 & 10 \\
\hline Sei & 2 & 2 & 4 \\
\hline Sette & 2 & 3 & 5 \\
\hline Otto & 1 & 0 & 1 \\
\hline Promossi nella secondaria di secondo grado, di cui: & 68 & 30 & 98 \\
\hline Liceo & 11 & 4 & 15 \\
\hline Istituto Tecnico & 44 & 18 & 62 \\
\hline Istituto Professionale & 10 & 6 & 16 \\
\hline CFP & 3 & 1 & 4 \\
\hline Scuola non specificata & 0 & 1 & 1 \\
\hline Giudizio sospeso & 46 & 37 & 83 \\
\hline Bocciato & 30 & 26 & 56 \\
\hline Liceo & 7 & 3 & 10 \\
\hline Istituto Tecnico & 16 & 21 & 37 \\
\hline Istituto Professionale & 7 & 2 & 9 \\
\hline Non disponibile & 3 & 6 & 9 \\
\hline Non scrutinato & 0 & 1 & 1 \\
\hline Trasferito & 1 & 0 & 1 \\
\hline Totale & 153 & 105 & 258 \\
\hline
\end{tabular}

Più complesso è risultato recuperare i dati dei ragazzi non seguiti. Alcuni cambi di referenti e di istituti non hanno permesso di recuperare tutti i dati e di tenere monitorati continuativamente nella seconda annualità gli studenti seguiti nel primo anno e poi ritirati. Non è stato quindi sempre possibile avere un ritorno sugli esiti di tutti gli alunni coinvolti inizialmente. Inoltre, alcuni operatori hanno avuto difficoltà a conoscere l'esito finale del secondo anno, e recuperare dalle scuole la votazione di giugno, e ancor di più, in caso di voto sospeso, l'esito della riparazione a settembre. Dei 116 ragazzi dei quali è stato possibile conoscere l'esito di fine anno, il $38 \%$ è stato promosso, poco più del $10 \%$ è stato rimandato, e circa il $30 \%$ ha avuto un esito negativo. I risultati sono quindi inferiori rispetto a quelli degli studenti seguiti anche nella seconda annualità.

Ciò testimonia, pur nella fragilità delle carriere scolastiche nella scuola secondaria di secondo grado, l'efficacia del dispositivo di tutoraggio e accompagnamento, che ha permesso di ottenere risultati più significativi tra $i$ ragazzi seguiti, rispetto a chi non è stato accompagnato anche nel secondo anno. I dati richiamano quindi la necessità di prevedere, nel momento così delicato di transizione alle superiori, interventi mirati per sostenere gli stu- 
denti di origine immigrata che entrano in un percorso scolastico assai più complesso e articolato rispetto alla media.

Tab. 15 - Esiti scolastici studenti non frequentanti e seguiti a distanza

\begin{tabular}{|l|r|r|r|}
\hline Esito scolastico giugno 2015 & F & M & Totale \\
\hline Promosso & 25 & 19 & 44 \\
\hline Bocciato & 18 & 18 & 36 \\
\hline Giudizio sospeso & 10 & 7 & 17 \\
\hline Trasferito & 3 & 4 & 7 \\
\hline Ritirato & 2 & 4 & 6 \\
\hline Ritorno al paese di origine & 1 & 2 & 3 \\
\hline Abbandono scolastico & 1 & 1 & 2 \\
\hline Non scrutinato & 0 & 1 & 1 \\
\hline Non disponibile & 34 & 53 & 87 \\
\hline Totale & $\mathbf{9 4}$ & $\mathbf{1 0 9}$ & $\mathbf{2 0 3}$ \\
\hline
\end{tabular}

\section{L'apprendimento dell'italiano}

Per concludere, diamo un'occhiata ai livelli linguistici in uscita. È stato possibile confrontare la competenza in italiano L2 iniziale e conclusiva di 349 studenti. Ecco di seguito la situazione finale registrata tra i ragazzi.

Graf. 9 - Livelli linguistici in uscita

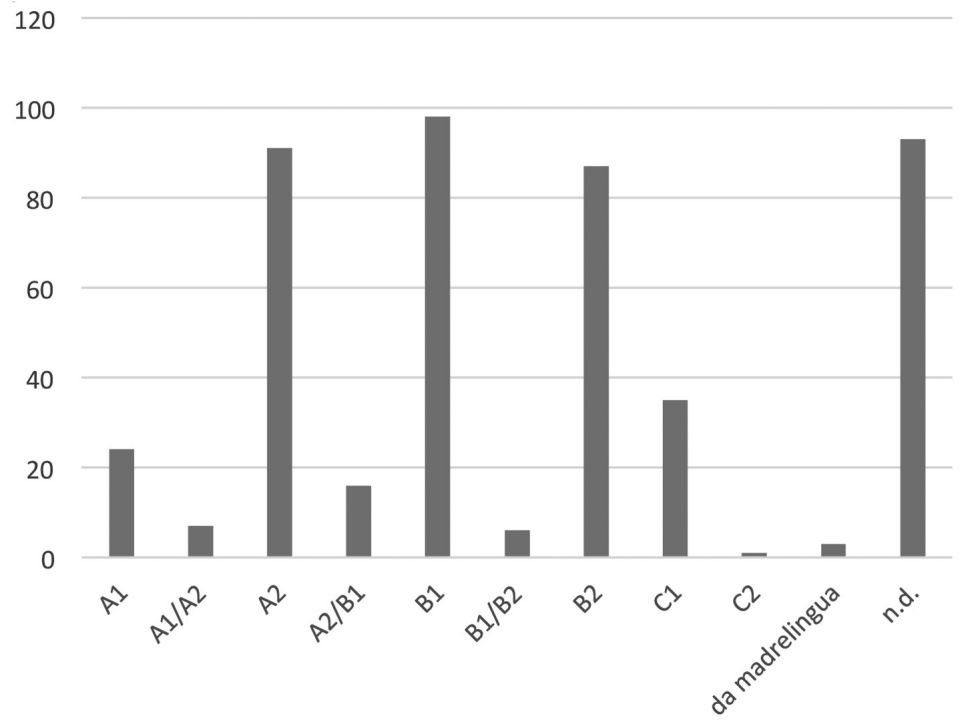


Proviamo a verificare le differenze tra il livello iniziale e quello conclusivo nei ragazzi che hanno frequentato il secondo anno: i dati sono disponibili per 187 studenti.

Tab. 16 - Confronto tra i livelli linguistici in entrata e uscita

\begin{tabular}{|c|c|c|c|c|}
\hline Livello iniziale & Livello finale & $\mathbf{F}$ & $\mathbf{M}$ & Totale \\
\hline $\mathrm{A} 0$ & $\mathrm{~A} 1$ & 1 & 2 & 3 \\
\hline A1 & $\mathrm{A} 1$ & 6 & 2 & 8 \\
\hline A1 & $\mathrm{A} 2$ & 13 & 6 & 19 \\
\hline A1 & B1 & 6 & 2 & 8 \\
\hline $\mathrm{A} 1$ & B2 & 2 & 1 & 3 \\
\hline A1 & C1 & 2 & 0 & 2 \\
\hline $\mathrm{A} 1 / \mathrm{A} 2$ & $\mathrm{~A} 1 / \mathrm{A} 2$ & 0 & 1 & 1 \\
\hline $\mathrm{A} 1 / \mathrm{A} 2$ & $\mathrm{~A} 2$ & 0 & 2 & 2 \\
\hline $\mathrm{A} 1 / \mathrm{A} 2$ & $\mathrm{~A} 2 / \mathrm{B} 1$ & 1 & 1 & 2 \\
\hline $\mathrm{A} 1 / \mathrm{A} 2$ & B1 & 1 & 0 & 1 \\
\hline $\mathrm{A} 2$ & $\mathrm{~A} 2$ & 17 & 17 & 34 \\
\hline $\mathrm{A} 2$ & $\mathrm{~A} 2 / \mathrm{B} 1$ & 1 & 1 & 2 \\
\hline $\mathrm{A} 2$ & B1 & 8 & 6 & 14 \\
\hline $\mathrm{A} 2$ & B2 & 7 & 0 & 7 \\
\hline $\mathrm{A} 2 / \mathrm{B} 1$ & $\mathrm{~A} 2 / \mathrm{B} 1$ & 5 & 2 & 7 \\
\hline $\mathrm{A} 2 / \mathrm{B} 1$ & B1 & 0 & 1 & 1 \\
\hline $\mathrm{A} 2 / \mathrm{B} 1$ & B1/B2 & 1 & 1 & 2 \\
\hline B1 & $\mathrm{B} 1 / \mathrm{B} 2$ & 0 & 1 & 1 \\
\hline $\mathrm{B} 1 / \mathrm{B} 2$ & $\mathrm{~B} 1 / \mathrm{B} 2$ & 2 & 1 & 3 \\
\hline B2 & $\mathrm{B} 2$ & 29 & 19 & 48 \\
\hline B2 & $\mathrm{C} 1$ & 3 & 2 & 5 \\
\hline $\mathrm{C} 1$ & $\mathrm{C} 1$ & 8 & 6 & 14 \\
\hline Totale & & 113 & 74 & 187 \\
\hline
\end{tabular}

I dati evidenziano per molti il mantenimento del livello in ingresso, anche se docenti e tutor confermano i miglioramenti sia nella produzione che nella comprensione da parte di quasi tutti gli studenti seguiti. I progressi si notano in particolare nel passaggio dal livello A1 ai successivi, soprattutto all'A2, soprattutto da parte delle ragazze, e dall'A2 al B1 e al B2. Il progetto conferma quindi l'importanza di un sostegno mirato all'apprendimento della lingua, che può aiutare i ragazzi non solo nella comunicazione orale 
ma anche nello studio delle discipline e nella conoscenza delle microlingue disciplinari. In sintesi, l'analisi dei dati ci permette di dire che, pur nella discontinuità e complessità dei percorsi, un affiancamento competente, nella didattica e nella relazione, rappresenta un efficace strumento a sostegno dei percorsi scolastici dei ragazzi di origine straniera.

\section{Alcune considerazioni finali}

Se proviamo a mettere a confronto gli esiti degli interventi nei due anni e cerchiamo di desumere alcune considerazioni finali in merito all'efficacia del progetto, possiamo trarre alcune conclusioni. Selezioniamo, tra i dati presentati in precedenza, solo quelli dei ragazzi effettivamente frequentanti, e di cui sono disponibili i risultati alla fine dell'annualità di riferimento, paragoniamoli a quelli dei ragazzi che non hanno più seguito nella seconda annualità, ma sono stati monitorati e dei quali è stato possibile conoscere con certezza i risultati.

Tab. 17 - Confronto degli esiti tra i ragazzi frequentanti e quelli non più frequentanti (monitorati a distanza)

\begin{tabular}{|c|c|c|c|c|c|c|}
\hline Esiti & $\begin{array}{c}\text { Ragazzi } \\
\text { frequentanti } \\
\text { I anno }\end{array}$ & $\%$ & $\begin{array}{c}\text { Ragazzi } \\
\text { frequentanti } \\
\text { II anno }\end{array}$ & $\%$ & $\begin{array}{c}\text { Ragazzi } \\
\text { monitorati } \\
\text { non più } \\
\text { frequentanti }\end{array}$ & $\%$ \\
\hline Promossi & 181 & $65,58 \%$ & 108 & $43,37 \%$ & 44 & $37,93 \%$ \\
\hline Rimandati & (85 su 159) & $(53,46 \%)$ & 83 & $33,33 \%$ & 17 & $14,66 \%$ \\
\hline Bocciati & 80 & $28,99 \%$ & 56 & $22,50 \%$ & 36 & $31,03 \%$ \\
\hline $\begin{array}{l}\text { Altro } \\
\text { (interruzione } \\
\text { di frequenza, } \\
\text { ritiro, rientro nel } \\
\text { Paese d'origine, } \\
\text { abbandono } \\
\text { scolastico) }\end{array}$ & 15 & $5,43 \%$ & 2 & $0,80 \%$ & 19 & $16,38 \%$ \\
\hline Totale & 276 & $100 \%$ & 249 & $100 \%$ & 116 & $100 \%$ \\
\hline
\end{tabular}

Nel primo anno abbiamo dati aggregati, perché è stato possibile sciogliere, come abbiamo visto, i giudizi sospesi relativi a chi era stato rimandato a settembre. Nel complesso, abbiamo quindi più del $65 \%$ di esito positivo, e più del $5 \%$ di interruzioni di frequenza scolastica non dovute $a$ bocciatura (ritiro, abbandono, rientro nel Paese d'origine...). Nel secondo 
anno, come dicevamo, non è stato possibile avere un ritorno in merito agli esiti di chi era stato rimandato a settembre, vista la conclusione del progetto a giugno. Se però, ipotizzando i risultati finali, dovessimo mantenere la media di promozioni sul numero dei rimandati a settembre (ricordiamo che nel primo anno era stata del 67\%), il tasso di promozione finale si alzerebbe al 65,46\%. Un dato ipotetico, ma abbastanza in linea con il primo anno. Tenendo poi conto che in questa seconda annualità $i$ ragazzi inseriti in terza media (e tutti promossi a fine anno) sono stati soltanto dieci, il dato sarebbe estremamente positivo, perché significherebbe un contributo davvero importante alla riuscita dei minori nei percorsi di scolarità superiore. Tale risultato positivo si conferma se mettiamo a confronto i ragazzi frequentanti con quelli che, accompagnati nel primo anno, non è stato possibile seguire nel secondo anno, ma dei quali siamo riusciti a sapere gli esiti della seconda annualità. In questo caso, i risultati sono significativamente inferiori: percentualmente più basso il numero di promossi, ma anche decisamente più elevate le bocciature, e una drastica riduzione dei rimandati. Oltre che una percentuale preoccupante di interruzioni di frequenza e abbandono scolastico. Questo testimonia, come si diceva, l'importanza di attivare dispositivi non contingenti ed estemporanei, ma più continuativi e costanti, in particolar modo nei momenti di passaggio. D'altra parte, sottolinea la funzionalità dei dispositivi di sostegno e integrazione ai percorsi scolastici in un contesto complesso e articolato come quello della scuola secondaria, in cui la competenza e la padronanza nello studio e l'approfondimento delle lingue disciplinari diventano indispensabili per la riuscita. Affiancare a strumenti più ordinari (il laboratorio mattutino, il doposcuola per l'accompagnamento ai compiti e al metodo di studio...) figure con una formazione specifica, motivate ed entusiaste come quelle dei tutor, ha permesso di conseguire risultati validi e importanti. Tanto più significativi se teniamo conto che i ragazzi segnalati dalle scuole, come dicevamo, non sono i più capaci e preparati, né i cosiddetti "studenti stranieri medi”, ma quelli con maggiori difficoltà e criticità. Docenti ed operatori delle scuole coinvolte, interpellati in merito all'utilità dell'intervento, hanno sottolineato come le azioni attivate abbiano permesso ai ragazzi più vulnerabili e a rischio di ottenere risultati positivi che senza il progetto sarebbe stato difficile ottenere. "Almeno una stella" ha saputo quindi consolidare ed implementare un modello di intervento diffuso, che, anche nei risultati, ha migliorato gli esiti del percorso scolastico e si è rivelato utile e funzionale al sostegno della riuscita scolastica dei minori di origine immigrata. 


\title{
5. Stare accanto. Modi e figure del tutoraggio
}

\author{
di Graziella Favaro
}

\section{1. "Stelle" sul cammino}

I progetti di tutoring o di mentoring, destinati in particolare ai minori che hanno una storia diretta o famigliare di migrazione, sono largamente diffusi in molti Paesi europei ed extraeuropei e hanno rivelato nel tempo la loro efficacia (Commissione Comunità Europee, 2008; Eurydice, 2009). Originatisi da idee lanciate e sperimentate dapprima in Israele, essi sono attualmente realizzati in maniera ampia negli Stati Uniti, nei Paesi del Nord Europa, in Olanda, in Germania. Citiamo, tra gli altri: la rete "Nightingale", che prende il nome dall'usignolo, il quale - si legge nella presentazione del progetto - canta solo quando si sente al sicuro, e che conta oggi una diffusione in otto contesti nazionali, dopo avere preso avvio in Svezia (a Malmö) e negli Stati Uniti. O ancora, il progetto "Goal", che può contare attualmente ad Amsterdam su circa 400 mentori e che è stato poi ripreso anche ad Amburgo e a Stoccolma, la rete europea Sirius che raggruppa enti, esperienze e progetti di diversi Paesi. In Olanda, vengono destinate da anni risorse economiche ingenti e continuative allo sviluppo di progetti di mentoring diffuso a scuola e nei quartieri.

Due ragioni strutturali e altrettanti modelli di riferimento stanno alla base delle azioni e delle iniziative che coinvolgono la figura dei mentori in altri Paesi. Da un lato, esse si sviluppano soprattutto nei contesti che hanno una storia consolidata di immigrazione e che, di conseguenza, possono contare su una radicata presenza di cittadini di origine straniera distribuiti su classi generazionali diverse. Si collocano inoltre in Paesi caratterizzati da forti concentrazioni di immigrati e dei loro figli in determinati quartieri e zone delle città. Dall'altro lato, esse si basano su una forte valorizzazione e un chiaro riconoscimento delle comunità straniere, delle diverse origini e delle "appartenenze" di ciascuno. Sulla base di questo convincimento, si ritiene in certi casi maggiormente efficace l'intervento diretto e duraturo di 
una figura di prossimità, che presenti tratti biografici e culturali di vicinanza e corrispondenza nei confronti dei destinatari, rispetto, ad esempio, al ruolo di un operatore dei servizi comuni.

In questa visione, gli elementi di somiglianza e di conseguente e possibile rispecchiamento possono diventare centrali nello sblocco di situazioni di difficoltà e ripiego che richiedono un affiancamento e un'attenzione mirata. I mentori inoltre agiscono quasi sempre entro luoghi neutri e informali, oppure negli spazi famigliari e nella dimora di coloro che sono da loro accompagnati e sostenuti.

Più simile al modello e all'impostazione francesi, la situazione italiana tende invece a sottolineare la necessità di una buona integrazione dei cittadini stranieri e dei loro figli, considerati come singoli individui e non come appartenenti a determinate comunità e gruppi. E questo cammino si deve compiere soprattutto entro i servizi e gli spazi comuni di tutti e per tutti. Scarso protagonismo è stato finora riconosciuto ai "rappresentanti etnici" in quanto tali, i quali possono tuttavia essere impiegati, se opportunamente formati, come mediatori linguistico-culturali, come risorsa al servizio di un servizio. Nella scuola, ad esempio, i mediatori linguistico-culturali vengono chiamati per fare da ponte, per organizzare la fase di accoglienza e di primo inserimento degli alunni neoarrivati, per tradurre messaggi e colloqui, per avvicinare la scuola alle famiglie immigrate e viceversa.

Un tratto che accomuna gli adolescenti immigrati che ricominciano la loro vita altrove e riprendono il cammino di apprendimento in un altro contesto è la condizione diffusa di solitudine che li costringe spesso a prendere decisioni cruciali senza poter contare su orientamenti, consigli, esperienze di altri. Sviluppano di conseguenza l'impressione di essersi "fatti da soli", come Beneduce segnala: "laddove mancano saldi punti di riferimento (simbolici, linguistici, sociali), cosa non infrequente quando il progetto migratorio della famiglia naufraga fra difficoltà e insuccessi, gli adolescenti immigrati sembrano confrontarsi con una singolare forma di solitudine e diventare quasi demiurghi di se stessi e del proprio destino" (Beneduce, 1998).

Anche di fronte alle richieste della scuola e alle fatiche di una scolarità che è segnata da una decisa discontinuità, gli adolescenti stranieri si trovano a doversi destreggiare fra cadute e riprese, fra la voglia di riuscire e la perdita delle illusioni e della motivazione, senza ancoraggi e punti di riferimento. Secondo Boris Cyrulnik (2005 e 2009), un bambino o un ragazzo che hanno sperimentato una situazione di rottura e un vissuto traumatico, per poter intraprendere un percorso di "neosviluppo resiliente", devono trovare sul loro cammino qualche stella: "Occorre che la società e la cultura abbiano predisposto intorno al minore qualche stella, ossia dei rapporti e delle relazioni che rappresentano dei tutori di resilienza". 
Chi può funzionare da "stella" nei confronti delle ragazze e dei ragazzi immigrati? Quali possono essere le figure che, all'interno o all'esterno della famiglia, li sostengono per un pezzo di strada, offrono ascolto ed empatia, li aiutano in maniera concreta e contribuiscono a riparare almeno un po' la fiducia in se stessi che nel frattempo si è affievolita?

Nel progetto che abbiamo voluto denominare proprio "Almeno una stella", abbiamo cercato di disseminare la rotta scolastica dei ragazzi neoarrivati con qualche "stella". Abbiamo affidato questo compito, oltre che agli insegnanti e agli educatori professionali, anche a un gruppo di tutor che hanno contribuito ad accogliere, almeno in parte, la vulnerabilità degli adolescenti stranieri affinché essa potesse trasformarsi, con il tempo e con attenzioni mirate, in una situazione più aperta e in un percorso di resilienza.

\section{Tra vicinanza e giuste distanze}

Il progetto "Almeno una stella" ha coinvolto nei due anni 131 tutor italiani e stranieri, figure di prossimità che hanno accompagnato i ragazzi immigrati nei momenti dedicati ai laboratori linguistici, allo studio e all'apprendimento, in generale. Più che di "mentori", si è trattato di tutor e di accompagnatori/facilitatori che hanno agito lungo il cammino della scolarità e negli spazi comuni dell'apprendimento. I due termini di mentoring e tutoring vengono spesso sovrapposti e usati come sinonimi, ma si riferiscono in realtà a figure e compiti non del tutto coincidenti e sovrapponibili. Il tutor opera all'interno di un'istituzione di educazione formale, nell'ambito di un progetto educativo specifico e definito e il suo intervento si concentra soprattutto sugli aspetti cognitivi e dell'apprendimento. Il mentore invece agisce anche in maniera autonoma, seppure sempre all'interno di un progetto, e soprattutto nelle situazioni informali. Il suo ruolo di guida e di modello di identificazione e riferimento positivi ha a che fare maggiormente con le componenti socio-emotive della storia personale e scolastica e con l'attesa di un cambiamento nel comportamento da parte del minore accompagnato.

Tutti i giovani tutor di "Almeno una stella" hanno operato all'interno delle istituzioni scolastiche e sempre in luoghi formali, accanto ai docenti e ai facilitatori linguistici, svolgendo quindi un ruolo di tutoraggio educativo nei confronti degli adolescenti accompagnati. Hanno assunto dunque un ruolo di "fratelli e sorelle maggiori", connotato ad un tempo, sia dalla vicinanza che da una certa, seppure contenuta, asimmetria e distanza. Essi sono infatti prossimi perché hanno attraversato in tempi abbastanza recenti le stesse difficoltà, hanno cercato risposta alle medesime domande, hanno 
proceduto lungo il cammino faticoso del diventare grandi altrove per tentativi ed errori, prove e riprove.

Essi sono tuttavia anche un po' distanti perché hanno un'età più matura, un'esperienza più elaborata che li ha già messi a confronto con le scelte, la definizione di sé, le sfide e gli atti di costruzione del futuro.

I punti di forza che possono rendere efficace la presenza di questa figura nei progetti rivolti agli adolescenti immigrati sono rintracciabili, oltre che nelle comuni biografie, che presentano tratti di corrispondenza e che rendono possibile il rispecchiamento, anche nella loro collocazione anagrafica. I tutor, studenti universitari o allievi frequentanti gli ultimi due anni delle scuole superiori, hanno un'età che va dai 18 ai 25 anni. Non sono quindi ancora adulti, ma non sono neppure dei pari, rispetto ai ragazzi che essi accompagnano. La differenza di età (cha va da 5 a 10 anni) li colloca in uno spazio intermedio e nella giusta distanza, sia rispetto agli adolescenti seguiti, che agli insegnanti e ai loro genitori. Questo ha permesso ai tutor di stabilire una relazione più aperta e autentica con i ragazzi e durante gli incontri hanno potuto emergere frammenti di storie, emozioni trattenute, domande che non avevano trovato fino a quel momento voce, interlocutori e ascolto.

Un tratto che caratterizza spesso le storie personali e scolastiche degli adolescenti immigrati è la perdita di fiducia nelle proprie capacità e il venir meno, di conseguenza, della motivazione ad apprendere. La sola presenza fisica dei tutor nello spazio dell'apprendimento e la visibilità di una storia che ha tratti di comunanza rispetto alla propria, rappresentano di per sé degli elementi positivi che sono generatori di cambiamenti. Esse propongono in maniera immediata un messaggio di speranza e apertura: i tutor rappresentano la prova che è possibile compiere il cammino nella nuova scuola e nella seconda lingua, che il disorientamento può essere superato e riparato e che ognuno può trovare la propria rotta, pur partendo da rive diverse.

Alla fine dell'esperienza, la maggior parte dei giovani tutor ha sottolineato, nella compilazione dei "diari di bordo" che hanno accompagnato la loro esperienza (un esempio di diario di bordo è allegato al capitolo), che una delle caratteristiche salienti di questo ruolo è la capacità di stare in ascolto, di cogliere segnali di apertura o di chiusura, di intercettare domande che non sempre trovano parole per esprimersi.

\section{Specchi e finestre}

La mia storia non è molto diversa dalla loro, perché anch'io mi sono trovata nella situazione simile e come loro ho dovuto affrontare molti ostacoli. Inserirsi in una nuova cultura diversa del tutto dalla proprio è molto difficile, per fortuna nel mio 
percorso scolastico ho trovato anch'io «una stella» che mi ha portato fin qui: l'insegnante di laboratorio italiano. Molte volte penso che senza di lei non sarei mai riuscita a continuare con successo il mio percorso (...) Avendo una storia uguale a loro, i ragazzi mi hanno fatto domande: da che Paese venissi, da quanto fossi qui, che scuole ho fatto e soprattutto si erano incuriositi della mia cultura perché indosso sempre i vestiti tradizionali del mio Paese. (Dal diario di A.N.)

Personalmente, mi sento quasi totalmente italiana, non solo perché posseggo la cittadinanza, ma anche perché la mia vita ormai l'ho vissuta interamente in Italia; infatti quando vado in Egitto, durante le vacanze estive per far visita ai miei parenti, mi sento come se fossi una turista in un Paese tuttavia familiare. Eppure mi sento appartenente anche alla cultura araba: la tradizione araba la respiro tutto i giorni in casa parlando arabo con i miei, guardando la tv satellitare che trasmette programmi in arabo, mangiando piatti tradizionali sia italiani che egiziani, e professando la religione islamica. (...) Essendo io stessa figlia di immigrati provenienti dall'Egitto, credo che i ragazzi e le ragazze immigrati siano persone molto forti e coraggiose, che non scelgono di lasciare il proprio Paese per capriccio, ma per varie necessità o motivi di tipo economico, sociale o politico. Lasciare il proprio Paese di origine per queste persone significa lasciare tutto ciò che conoscono e hanno di familiare, per avventurarsi in un Paese spesso sconosciuto con la speranza di un futuro migliore. È questo che vedo nei ragazzi che ho seguito ed è quello che vedo in me stessa. (Dal diario di S.E.S.)

I frammenti di racconto dell'esperienza dei tutor milanesi, raccolta nei "diari di bordo", ci introduce dentro il progetto, le sue tappe, le sfide incontrate sul cammino e ci permette di cogliere i vissuti dei tutor, la loro rappresentazione dei ragazzi seguiti e di registrare i passaggi, i cambiamenti, le consapevolezze.

Nella fase iniziale, la chiarezza del compito e degli obiettivi (preparazione dell'esame di terza media e aiuto allo studio) ha permesso ai giovani tutor di collocarsi e di definire con qualche precisione il loro ruolo. E ha facilitato il loro riconoscimento da parte degli alunni accompagnati: "è uno più grande di me che mi aiuta a studiare". Le emozioni registrate da parte degli uni e degli altri in questa fase avevano a che fare con i timori di insicurezza e inadeguatezza da parte dei tutor: ce la farò? Sarò all'altezza? Ma anche con il loro entusiasmo, la curiosità, la voglia di cimentarsi e di provarsi in una nuova esperienza. Da parte dei ragazzi seguiti, i vissuti, espressi o impliciti, hanno riguardato inizialmente una certa chiusura e diffidenza nei confronti di figure dai contorni professionali non ben definiti, rinforzate dagli atteggiamenti frequenti nei ragazzi neo arrivati di cercare di non farsi notare, di diventare essere "invisibili".

E tuttavia, fin dai momenti iniziali si è attivato un meccanismo di reciproco rispecchiamento. I tutor hanno riconosciuto nelle storie, spesso travagliate delle ragazze e dei ragazzi seguiti, come in uno specchio, vicende 
e vissuti della loro stessa storia. Gli adolescenti accompagnati, da parte loro, hanno trovato nei giovani tutor la possibilità non solo di essere aiutati, ma anche di essere visti in modo diverso da una finestra più familiare. Di ritrovare uno sguardo su di sé più coinvolto ed empatico, come i frammenti di diario riportati sopra sottolineano.

Le modalità di rispecchiamento, pur mantenendo la giusta distanza, nelle storie di adolescenza migrante si ritrova anche nelle scritture che $\mathrm{i}$ tutor fanno dei ragazzi seguiti. In questi profili, vengono raccontate le vicende personali e scolastiche, le difficoltà e le conquiste, gli atteggiamenti e i cambiamenti. Ma vengono sottolineati anche gli aspetti che hanno a che fare con le emozioni, i talenti, gli eventi imprevisti che sono all'origine di blocchi e "cadute", oppure di sblocchi e di ricominciamenti. Troviamo un esempio di questa descrizione partecipata nel racconto che i tutor torinesi hanno fatto dei "loro" ragazzi seguiti nei due anni e degli esiti del progetto.

\section{La storia di $D$.}

D. ha 14 anni ed è appena arrivata dall'Albania. È venuta in Italia per studiare, poiché è molto motivata ed abituata ad eccellere a scuola. La sua famiglia è rimasta in Albania, mentre lei a Torino vive con la zia. Frequenta il liceo scientifico "Einstein". Il primo giorno che viene ad iscriversi al Centro accompagnata dalla zia, è molto in difficoltà: alle problematiche linguistiche incontrate a scuola, si sommano le ansie legate al fatto di non essere più la prima della classe, al non comprendere bene e al non poter stare al passo con i compagni. Inoltre, D. sente il "peso" del sacrificio della sua famiglia, che l'ha mandata a studiare in Italia e non vuole deludere in nessun modo i suoi genitori.

D. frequenta assiduamente per tutto l'anno sia il corso di italiano, sia i momenti di sostegno allo studio. È determinata e motivata, si organizza e si gestisce autonomamente cercando di svolgere la maggior parte delle materie in cui ha più difficoltà con i tutor e chiedendo aiuto sugli aspetti della lingua che ancora non padroneggia o che non comprende.

Partecipa portando un suo scritto in albanese e in italiano alla "Giornata per la promozione della Lingua madre" insieme ad altri ragazzi del Centro Interculturale. I risultati scolastici progressivamente migliorano, grazie al supporto costante ricevuto dai tutor di "Almeno una stella" e, naturalmente, per merito della sua tenacia e forza di volontà. Alla fine dell'anno, D. viene promossa al secondo anno del liceo scientifico con 7.

Il progetto ha svolto un'altra importantissima funzione per una ragazzina appena arrivata in Italia: ha risposto al bisogno, centrale di ogni adolescente, e soprattutto per un adolescente straniero appena arrivato in un nuovo Paese e perciò senza amici, di relazionarsi con un gruppo di pari, di costruire nuove relazioni di amicizia. D. ha trovato le "cure" costanti e stabili dei tutor, che hanno risposto al bisogno di attenzione e di affetto di una ragazzina che ha lasciato lontano i propri punti di riferimento. 


\section{La storia di A.}

A. ha 17 anni ed è arrivato in Italia con la madre nel 2010. È un ragazzo introverso, ritenuto dagli insegnanti "difficile" e "incontenibile". A. trascorre molto tempo in strada: ha imparato a cavarsela da solo, poiché la mamma lavora in una casa di riposo durante tutta la settimana. Quando arriva al Centro Interculturale nel primo anno di progetto, non parla quasi con nessuno, tranne che con qualche suo compagno di classe; tende ad isolarsi e a rifuggire i momenti di aggregazione; lega però da subito con il tutor di riferimento, anche se non ha molta voglia di studiare e di conseguenza non viene promosso.

Nella seconda annualità di progetto, gli educatori e i tutor costruiscono fin da subito un forte legame con lui, dedicandogli attenzioni e momenti di ascolto: ciò permette ad. A. di aprirsi, di mostrarsi senza paura ai tutor e agli altri ragazzi. Nel secondo anno di "Almeno una stella" infatti avviene una metamorfosi: A. chiede lui stesso di studiare, di essere aiutato nei compiti e di partecipare alle attività aggregative. Alla fine dell'anno, $\mathrm{A}$. ha un solo debito di inglese, è più sereno $\mathrm{e}$ ha trovato un luogo di appartenenza in cui sta bene.

\section{La storia di $M$.}

M. ha 17 anni, è marocchina ed è in Italia da alcuni anni. Ha molte difficoltà di inserimento a scuola, non riesce a stare al passo con gli altri, si relaziona poco con i compagni e i professori perché è timida e, soprattutto, ha molta paura di essere presa in giro quando parla italiano, anche se lo parla ormai molto bene. Quest'anno riesce a costruire molte relazioni di amicizia con gli altri ragazzi che partecipano al progetto, perché si sente simile a loro, con le stesse paure e difficoltà nello studio. M., con l'aiuto della sua tutor, recupera 6 materie insufficienti e a fine hanno ha solo un debito in storia.

\section{Ritratti di tutor}

Come abbiamo visto nel capitolo dedicato alla descrizione del progetto, ad "Almeno una stella" hanno partecipato nei due anni 131 tutor. Vediamo alcune caratteristiche personali e le loro modalità di lavoro e di partecipazione.

\section{Distribuzione e permanenza}

Per quanto riguarda la distribuzione territoriale i tutor erano collocati nel modo seguente:

- 23 in ciascuna città a: Milano, Torino, Arezzo;

- 25 a Bologna;

- 26 nella provincia di Trento;

- 11 nelle città friulane. 
Dei 131 tutor, 62 hanno partecipato al progetto per tutta la durata dei due anni; 28 sono intervenuti solo durante il primo anno e 41 solo nel secondo anno di attività. A Milano e a Torino si sono registrati i tassi di stabilità maggiore: in entrambi i casi, 17 tutor sui 20 inizialmente coinvolti hanno partecipato per tutta la durata del progetto. A Bologna, vi è stata una buona tenuta: 15 sui 20 presenti nella fase iniziale hanno completato l'impegno biennale, mentre ad Arezzo, sui 23 tutor coinvolti nel progetto, 10 hanno condotto l'intervento in entrambi gli anni. Nell'esperienza trentina e friulana, si è registrato invece un forte avvicendamento ma era un dato già previsto in partenza. Nelle due realtà infatti - che partecipavano al progetto in modo volontario senza beneficiare di alcun sostegno finanziario - si era deciso fin dall'inizio di chiedere ai tutor un impegno di un solo anno (nei due territori afferenti, i tutor non hanno ricevuto nessun rimborso delle spese, né un riconoscimento in termini di crediti universitari). Nella provincia di Trento, i giovani tutor coinvolti sono stati 26 e solo 3 hanno partecipato sia il primo che il secondo anno di progetto. A Udine e a Pordenone, i tutor sono stati 11, tutti con un interventi annuo: 4 coinvolti il primo anno e 7 il secondo anno.

Tab. 1 - I tutor: distribuzione e partecipazione

\begin{tabular}{|l|c|c|c|c|}
\hline Città & N. totale & $\begin{array}{c}\text { Entrambi } \\
\text { gli anni }\end{array}$ & I anno & II anno \\
\hline Milano & 23 & 17 & 3 & 3 \\
\hline Torino & 23 & 17 & 3 & 3 \\
\hline Bologna & 25 & 15 & 5 & 5 \\
\hline Arezzo & 23 & 10 & 3 & 10 \\
\hline Trento & 26 & 3 & 10 & 13 \\
\hline Udine e Pordenone & 11 & 0 & 4 & 7 \\
\hline Totale & $\mathbf{1 3 1}$ & $\mathbf{6 2}$ & $\mathbf{2 8}$ & $\mathbf{4 1}$ \\
\hline
\end{tabular}

\section{Soprattutto donne, soprattutto studenti universitari}

Il ritratto più diffuso dei tutor vede come caratteristiche prevalenti: una forte presenza femminile, la provenienza universitaria da facoltà e corsi di laurea a carattere umanistico, un'età relativamente bassa.

Fra i 131 tutor, le giovani donne sono state la grande maggioranza: 102 ragazze, pari al $78 \%$, e 29 ragazzi.

Il $70 \%$ proveniva dall'università (91 tutor), mentre 40 frequentavano gli ultimi anni della scuola secondaria di secondo grado. In particolare, a 
Milano i tutor erano tutti studenti universitari, così come a Udine e Pordenone. A Torino vi era la netta prevalenza degli universitari (20 su 23), così come a Trento (23 su 26). Ad Arezzo, la collocazione negli studi era invece mista (14 universitari e 9 studenti di scuola superiore), mentre a Bologna i tutor erano tutti studenti frequentanti gli istituti di istruzione superiore. Per quanto riguarda l'età, la grande maggioranza dei tutor si collocava nella fascia tra i 18 e i 25 anni (72\%), mentre il $28 \%$ aveva più di 25 anni.

Graf. 1 - Le età dei tutor

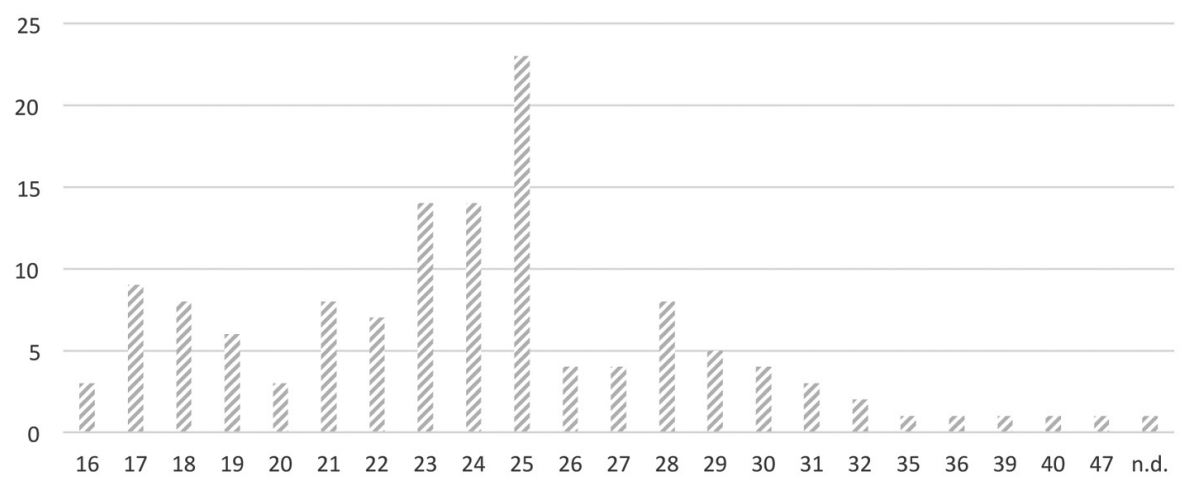

\section{Nazionalità e percorsi di migrazione}

Il progetto "Almeno una stella" mirava a coinvolgere in maniera privilegiata giovani tutor stranieri o di origine straniera, con una storia diretta e familiare di migrazione. Questo per consentire una maggiore vicinanza quanto a vissuti ed esperienza personale, biografie linguistiche, percorsi scolastici interrotti e ripresi altrove. Al termine dei colloqui e della selezione, il gruppo dei tutor è risultato composto da 70 giovani italiani e 61 stranieri o con doppia nazionalità. La situazione era inoltre diversa da città a città: a Torino, ad esempio, i tutor erano per lo più stranieri o di origine straniera, mentre a Trento e nelle città friulane si trattava solo o in prevalenza di studenti italiani.

Le provenienze nazionali registrate tra i 61 tutor con background migratorio rispecchiavano, sia le caratteristiche dell'immigrazione straniera in Italia, che le specificità che si registrano nelle diverse città. Fra i tutor stranieri, si nota la prevalenza di marocchini (7), seguiti dai peruviani (5), dai rumeni, moldavi e pachistani (4 per ciascun Paese); dai polacchi e tunisini (3 per ciascun Paese). Coloro che hanno la doppia cittadinanza (10) sono 
per lo più italo-marocchini (3), italo-tunisini e italo-eritrei (2 per ciascun Paese).

Tab. 2 - Le nazionalità

\begin{tabular}{|l|c|}
\hline Nazionalità & N. \\
\hline Albania & 1 \\
\hline Bangladesh & 1 \\
\hline Bielorussia & 2 \\
\hline Camerun & 2 \\
\hline Cina & 1 \\
\hline Congo & 2 \\
\hline Costa d' Avorio & 1 \\
\hline Croazia & 1 \\
\hline Ecuador & 1 \\
\hline Egitto & 1 \\
\hline Filippine & 2 \\
\hline Italia & 70 \\
\hline Macedonia & 1 \\
\hline Marocco & 7 \\
\hline Messico & 1 \\
\hline Moldavia & 4 \\
\hline Nigeria & 2 \\
\hline Pakistan & 4 \\
\hline Perù & 5 \\
\hline Polonia & 3 \\
\hline Romania & 4 \\
\hline Sri Lanka & 1 \\
\hline Tunisia & 3 \\
\hline Ucraina & 1 \\
\hline Italia-America & 1 \\
\hline Italia-Uruguay & 1 \\
\hline Italia-Tunisia & 2 \\
\hline Italia-Marocco & 3 \\
\hline Italia-India & 1 \\
\hline Italia-Eritrea & 2 \\
\hline Totale & 1 \\
\hline & \\
\hline & 1 \\
\hline & 1 \\
\hline & 1 \\
\hline & \\
\hline
\end{tabular}

Nella Tab. 3 riportiamo in sintesi le caratteristiche dei tutor, distinguendo tra i sei territori coinvolti. 
Tab. 3 - Le caratteristiche dei tutor

\begin{tabular}{|c|c|c|c|c|c|c|}
\hline Città & N. & $\mathbf{M} / \mathrm{F}$ & Nazionalità & Età & $\begin{array}{l}\text { Provenienza } \\
\text { scolastica }\end{array}$ & $\begin{array}{c}\text { Modalità } \\
\text { partecipazione }\end{array}$ \\
\hline Milano & 23 & $\begin{array}{l}20 \mathrm{~F} \\
3 \mathrm{M}\end{array}$ & $\begin{array}{l}18 \text { italiani } \\
5 \text { stranieri }\end{array}$ & $20-27$ & università & $\begin{array}{c}17 \text { entrambi } \\
3 \text { I anno } \\
3 \text { II anno }\end{array}$ \\
\hline Torino & 23 & $\begin{array}{l}13 \mathrm{~F} \\
10 \mathrm{M}\end{array}$ & $\begin{array}{c}10 \text { italiani } \\
13 \text { stranieri }\end{array}$ & $23-32$ & università & $\begin{array}{c}17 \text { entrambi } \\
3 \text { I anno } \\
3 \text { II anno }\end{array}$ \\
\hline Bologna & 25 & $\begin{array}{c}24 \mathrm{~F} \\
1 \mathrm{M}\end{array}$ & $\begin{array}{c}3 \text { italiani } \\
22 \text { stranieri }\end{array}$ & $16-19$ & $\begin{array}{l}\text { scuola secondaria } \\
\text { di secondo grado }\end{array}$ & $\begin{array}{l}15 \text { entrambi } \\
5 \text { I anno } \\
5 \text { II anno }\end{array}$ \\
\hline Arezzo & 23 & $\begin{array}{l}18 \mathrm{~F} \\
5 \mathrm{M}\end{array}$ & $\begin{array}{c}8 \text { italiani } \\
15 \text { stranieri }\end{array}$ & $18-35$ & $\begin{array}{c}4 \text { scuola secondaria } \\
\text { di secondo grado } \\
19 \text { università } \\
\end{array}$ & $\begin{array}{c}10 \text { entrambi } \\
3 \text { I anno } \\
10 \text { II anno } \\
\end{array}$ \\
\hline Trento & 26 & $\begin{array}{l}19 \mathrm{~F} \\
7 \mathrm{M}\end{array}$ & $\begin{array}{l}21 \text { italiani } \\
5 \text { stranieri }\end{array}$ & $23-40$ & università & $\begin{array}{c}3 \text { entrambi } \\
10 \text { I anno } \\
13 \text { II anno }\end{array}$ \\
\hline $\begin{array}{l}\text { Udine e } \\
\text { Pordenone }\end{array}$ & 11 & $\begin{array}{l}7 \mathrm{~F} \\
3 \mathrm{M}\end{array}$ & 11 italiani & $21-26$ & università & $\begin{array}{l}4 \text { I anno } \\
7 \text { II anno }\end{array}$ \\
\hline
\end{tabular}

\section{I punti di forza del tutoraggio}

Quali sono i punti di forza che fanno sì che la presenza dei tutor possa funzionare spesso come occasione di sblocco, di ri-motivazione, di ri-orientamento efficace? Come abbiamo visto, le loro caratteristiche personali, che li rendono "prossimi" ai ragazzi accompagnati, l'eterogeneità delle provenienze e delle storie, la competenza plurilingue fanno sì che essi possano giocare un ruolo positivo come risorsa per una buona inclusione.

Di seguito, ecco i punti di forza che riteniamo importanti nei tutor a partire dalla sperimentazione del progetto "Almeno una stella".

\section{I punti di forza}

\section{- La vicinanza anagrafica}

I tutor si collocano in un'età vicina, ma non pari, rispetto a quella dei ragazzi seguiti: rispetto a loro, non sono infatti né compagni né adulti. La distanza anagrafica potrebbe essere compresa tra i 5 e i 10 anni.

\section{- Un'appartenenza plurale}

È un elemento estremamente positivo il fatto che il gruppo dei tutor sia multiculturale e internazionale e che comprenda, accanto a giovani italiani nativi, anche stranieri che hanno vissuto direttamente la migrazio- 
ne, giovani stranieri di "seconda generazione", giovani che provengono dall'adozione internazionale e italiani di origine straniera.

\section{- La mescolanza di genere}

II gruppo dei tutor dovrebbe essere misto anche per genere, per rendere possibile la ricchezza e la complessità relazionale che si genera dai comuni interessi, dalle sensibilità e dalle vicende personali che sono più vicine e affini per genere.

\section{- La prossimità linguistica e il plurilinguismo}

La presenza nel gruppo di giovani bi o plurilingui è un elemento immediato di valorizzazione delle lingue d'origine dei ragazzi stranieri; prefigura inoltre la possibilità concreta di un cammino di apprendimento linguistico in italiano positivo e composito ed è un importante segno, simbolico e reale, di riconoscimento e di inclusione.

\section{- Linguaggi comuni}

I tutor condividono con gli adolescenti l'uso più fluido e agile di canali e strumenti multimediali e dei social network: questo si rivela positivo, sia nel lavoro didattico e dell'apprendimento, che nelle possibilità di approfondire e continuare le relazioni a distanza.

\section{- Il percorso formativo}

I tutor possono essere studenti inseriti nelle classi finali della scuola secondaria di secondo grado o frequentanti corsi di studio universitari. Alla luce dell'esperienza di "Almeno una stella", si osserva che questi ultimi hanno, in genere, una maggiore autorevolezza, una disponibilità più ampia e possono disporre di tempi più dilatati da dedicare all'esperienza.

\section{- Il riconoscimento formale}

L'impegno svolto come tutor nei progetti rivolti agli adolescenti immigrati deve essere riconosciuto dalle università e dalle scuole di provenienza in maniera formale, sia in termini di crediti, sia come parte del percorso di tirocinio o di stage.

\section{- La valorizzazione delle esperienze educative e di volontariato}

II lavoro di tutoraggio nei confronti di ragazzi più piccoli rappresenta un'esperienza importante e coinvolgente di assunzione di una responsabilità educativa diretta, di cura degli altri ed è un esercizio concreto di "cittadinanza attiva". Spesso chi si propone per questo ruolo ha avuto in precedenza esperienze di volontariato, di lavoro educativo informale o di servizio civile che vanno riconosciute e valorizzate.

- Un patto di tutoraggio

I tutor agiscono negli spazi e nei tempi scolastici o extrascolastici, nell'ambito di un progetto educativo definito e accanto a docenti specialisti. L'impegno annuo è di circa 80 ore (circa 2 ore la settimana), compresi i momenti iniziali della formazione e del monitoraggio in itinere. Tutti i soggetti e i partner coinvolti dovrebbero condividere e sottoscrivere un "patto di tutoraggio" che chiarisce gli obiettivi, i tempi, i ruoli. 


\section{Modelli diversi nelle città}

Due erano gli obiettivi posti al centro del progetto "Almeno una stella" ed erano indirizzati sia ai destinatari delle azioni che agli attori delle stesse. Da un lato, si intendeva agire con dispositivi mirati per sostenere i percorsi scolastici degli adolescenti neo arrivati, o di recente immigrazione, inseriti nella scuola secondaria di primo e di secondo grado. Dall'altro lato, il secondo obiettivo centrale riguardava la sperimentazione del dispositivo di tutoring e della figura dei tutor come accompagnatori e facilitatori del cammino di inserimento a scuola e nella città, come figure di prossimità in grado di svolgere un ruolo positivo di aiuto, guida e modello di identificazione per gli adolescenti stranieri.

Pur nella esplicita condivisione dei due obiettivi, ogni città ha declinato e organizzato le azioni in modo diverso sulla base dei bisogni e delle richieste delle scuole, delle priorità individuate, delle specificità locali.

Di seguito, una descrizione sintetica delle azioni e delle specificità locali riguardanti, in maniera particolare, il ruolo dei tutor e le condizioni del tutoraggio (la descrizione puntuale del progetto nelle varie città è contenuto nel cap. 2).

\section{A Milano, i tutor nelle scuole}

Il progetto milanese si è articolato in due fasi distinte: il primo anno è stato condotto nelle scuole secondarie di primo grado, con l'obiettivo centrale riferito alla preparazione all'esame di terza media. Il secondo anno si è rivolto alle scuole superiori, privilegiando gli istituti nei quasi si erano maggiormente iscritti gli allievi stranieri coinvolti il primo anno, con l'obiettivo di sostenere il passaggio di scuola e il delicato momento delle scelte scolastiche e dell'avvio dell'istruzione superiore. I tutor milanesi, tutti provenienti da studi universitari, hanno quindi assunto soprattutto un ruolo di aiuto allo studio, sostegno e guida e hanno operato negli spazi delle scuole affiancando i docenti dei laboratori.

\section{A Torino, i tutor al centro}

A Torino i destinatari delle azioni sono stati individuati negli studenti inseriti nel biennio delle scuole secondarie di secondo grado e prevedevano l'aiuto allo studio.

Le azioni di sostegno si sono svolte presso la sede del Centro Interculturale: sulla base di accordi con le scuole superiori della zona, gli studenti si sono recati al Centro in tempo extrascolastico. Il ruolo dei tutor torinesi era duplice. Da un lato, prevedeva il sostegno individuale allo studio entro uno spazio di "doposcuola" centralizzato; dall'altro lato, i tutor hanno 
organizzato uscite nella città con gli adolescenti stranieri per far loro conoscere i luoghi significativi della cultura, della vita pubblica, dell'aggregazione.

\section{A Bologna, i compagni accoglienti}

Anche nella città di Bologna, il progetto ha visto come destinatari gli studenti delle scuole secondarie di secondo grado, inseriti nel biennio e per lo più neo arrivati. In questo caso, anche i tutor erano studenti della scuola superiore, frequentanti gli ultimi anni delle stesse scuole coinvolte. Si è trattato quindi di un dispositivo di tutoring interno alle scuole, che ha visto gli studenti degli ultimi anni agire nel ruolo di "compagni accoglienti" nei confronti dei più piccoli. In questa funzione, i più grandi hanno fornito certamente anche un aiuto nello studio, ma hanno soprattutto proposto un sostegno relazionale e motivazionale a coloro che attraversavano una fase di disorientamento e di spaesamento.

\section{Ad Arezzo, i tutor di doposcuola}

Ad Arezzo, gli alunni destinatari delle azioni erano inseriti nelle scuole secondarie di primo e di secondo grado. Il gruppo dei tutor, composto da studenti di scuola superiore e da universitari, ha affiancato gli insegnanti negli spazi di doposcuola localizzati, sia presso le scuole, sia presso la sede centralizzata della Casa delle culture del mondo. Ad ogni tutor sono stati abbinati due adolescenti stranieri.

\section{Nelle città friulane, i tutor per l'esame}

Il progetto condotto nelle città friulane ha replicato nei due anni le impostazioni e gli obiettivi.

Sia il primo che il secondo anno, si è deciso di sostenere gli alunni di recente immigrazione nella preparazione all'esame di terza media.

I tutor, tutti studenti universitari, hanno affiancato i ragazzi, in tempo extrascolastico e in accordo con gli insegnanti, sia per aiutarli nella produzione di testi scritti, sia per dare loro maggiori strumenti per sostenere il colloquio orale.

\section{A Trento, il patto di tutoraggio}

Nelle scuole della Provincia di Trento, il progetto ha coinvolto sia le scuole secondarie di primo grado, sia le scuole superiori. Anche in questo territorio, gli obiettivi scolastici, relazionali, di facilitazione alla socializzazione si sono intrecciati fra loro. In particolare, i tutor hanno accompagnato gli adolescenti stranieri a scoprire e a usare le opportunità del territorio 
a carattere ludico, aggregativo, sportivo. Uno strumento importante, predisposto durante le azioni del progetto, è stato il "patto di tutoraggio" nel quale sono stati indicati in maniera chiara i tempi, i luoghi e le attività previsti, in modo tale che tutti i soggetti coinvolti (adolescenti, famiglie, tutor, insegnanti delle scuole) ne fossero informati e corresponsabili.

\section{In ascolto delle storie}

Che cosa ha fatto il tutor, sia negli spazi e nei tempi condotti dentro la scuola, sia nei laboratori realizzati in luoghi e tempi extrascolastici?

Come abbiamo visto, nelle diverse situazioni e città, si è osservata l'assunzione di compiti e lo svolgersi di funzioni diverse e sovrapposte, ora a carattere strettamente scolastico e legate all'aiuto allo studio, ora di tipo più informale e attente alle relazioni. L'accentuazione dell'una o dell'altra forma di tutoraggio - che comunque mai possono essere del tutto distinte e separate - dipendeva da fattori diversi, quali: le caratteristiche dell'insegnante di riferimento; la fisionomia e le storie dei ragazzi; i bisogni linguistici e scolastici che essi esprimevano; il luogo nel quale è stato condotto il laboratorio e il tempo che esso occupava, scolastico o extrascolastico.

In generale, possiamo notare che il ruolo dei tutor è stato più "scolastico" e mirato al compito durante il primo anno, condotto all'interno dei laboratori organizzati dentro le scuole medie e mirati in maniera esplicita alla preparazione dei ragazzi per affrontare l'esame finale. Nel secondo anno, il loro ruolo è diventato, in genere, più articolato, sia perché l'esperienza aveva nel frattempo contribuito a dare spessore e visibilità a questa nuova figura di prossimità dai contorni prima ancora incerti, sia perché le attività di accompagnamento si sono svolte spesso anche in luoghi non scolastici, in tempi pomeridiani e con gruppi di ragazzi che in parte già si conoscevano.

Nella descrizione delle azioni svolte durante cinque giorni di attività, scelti a caso, che ogni tutor aveva il compito di osservare e raccontare, compaiono dunque funzioni diverse. Esse hanno a che fare, ora con la vicinanza relazionale, ora con l'aiuto allo studio e con compiti più definiti.

In ogni caso, la presenza dei tutor ha contribuito a creare un quadro educativo più aperto, inclusivo e meno distante, dentro il quale hanno avuto modo di manifestarsi domande, racconti e anche talenti nascosti.

Ecco come i tutor hanno descritto nei loro diari di bordo il loro ruolo. 


\section{Un tutor ha una funzione di...}

- Protezione e sblocco

Racconta la propria storia di integrazione.

Sollecita il racconto dei ragazzi.

Protegge la fragilità dei ragazzi migranti.

Li aiuta a individuare il proprio Nord.

Crea un'alleanza positiva.

Crea le occasioni perché i ragazzi possano parlare di sé e della loro storia.

Stabilisce una relazione più confidenziale grazie alla stessa lingua.

Vive un territorio comune a quelli dei ragazzi perché ne condivide le parole.

Si mette in gioco in prima persona.

Tenta di rompere il ghiaccio.

Si mette in ascolto.

- Aiuto e sostegno scolastico

Spiega le consegne e le richieste.

Aiuta a fare i compiti, a scrivere, a studiare.

Media tra i ragazzi e i contenuti da imparare.

Spiega come si fa una "scaletta", come si mettono in ordine le idee.

Sostiene l'apprendimento dell'italiano nei momenti di laboratorio.

Cerca di creare migliori condizioni di studio, anche a due, e in maniera cooperativa.

Sollecita l'autocorrezione.

Propone un metodo di studio, anche a partire dalla propria esperienza.

Cerca di coinvolgere gli alunni più apatici e demotivati.

- Supporto e apertura

Cerca di creare un rapporto bilanciato: né troppo spostato verso gli insegnanti né troppo vicino ai ragazzi.

Attiva con il tempo uno scambio reciproco, tra sé e i ragazzi seguiti.

Contribuisce a far riconoscere i talenti e le capacità dei ragazzi stranieri al di là delle loro difficoltà linguistiche.

Cerca di rompere il ruolo passivo di molti ragazzi stranieri che stanno in classe come dei "fantasmi".

Cerca di aiutarli, coinvolgendoli, sostenendoli e incoraggiandoli con affetto e con equilibrio.

Passa il messaggio che "c'è, che è presente al loro fianco, che possono contare sul suo aiuto".

Accompagna nel percorso di studi, ma anche nella conoscenza del quartiere e della città.

Apre degli spiragli sul futuro.

Il tutor agisce su due livelli: come figura semiprofessionale (è un primo passo per diventarlo), in quanto risponde a esigenze scolastiche; come aiuto alla relazione perché cerca di proteggere le fragilità di quelli più piccoli di lui. 


\section{Le fasi del tutoraggio}

\section{In punta di piedi: le mosse iniziali}

Primo incontro con i ragazzi, c'è molto imbarazzo da parte dei ragazzi che bisbigliano e ridacchiano tra di loro nelle loro lingue, imbarazzo che viene ricambiato da parte nostra mentre le più disparate domande ci assediano: "Ce la farò? Sarò d'aiuto a questi ragazzi? Riuscirò a creare con loro un buon rapporto di fiducia?". Ed ecco che finalmente iniziamo con un giro di presentazione, i ragazzi timidamente dicono il loro nome, la loro età, da dove vengono, qualcuno si sforza di aggiungere quale sport preferisce o che tipo di musica ascolta. Poi arriva il nostro turno, ci apriamo e cerchiamo di dire quante più cose possibili sul nostro conto per cercare di far sciogliere quel muro di ghiaccio che si era eretto tra noi non appena varcata la porta, sembriamo riuscirci, ecco le prime domande e quelli che prima erano dei sorrisini nascosti adesso diventano delle risate condivise (...). Ogni sorta di formalità è ormai abbandonata, quando i ragazzi conversano tra loro in tagalog io li riprendo in siciliano, facendo capire loro quanto sia brutto essere privati della possibilità di interagire e pian piano abbandonano questa abitudine. (Dal diario di D.C.)

Ho cercato di presentarmi subito come un appoggio per loro cercando di trasmettere il fatto che, non essendo una professoressa, non li avrei giudicati in nessun modo, e che con me avrebbero potuto esprimersi liberamente, sia rispetto ai contenuti, sia rispetto alla forma. Erano ammessi errori di lingua su cui poi avremmo lavorato insieme. (Dal diario di C.M.)

Qualche ragazzo cinese ha paura di sbagliare, di essere preso in giro, di non farsi capire, per questo alcuni di loro non vogliono fare amicizia con i compagni italiani. Le loro insegnanti e i loro compagni non capiscono le difficoltà dei ragazzi cinesi. I cinesi si sentono isolati, ma in realtà non è che non vogliano comunicare con loro, e che non sanno parlare l'italiano. Solo in alcuni momenti riescono a capire la lingua e a parlare, oppure a comunicare con me in cinese: ed è in quei momenti che hanno delle idee fantastiche. (Dal diario di L.C.)

L'avvio di un'attività comune e l'esordio di una relazione educativa sono sempre momenti delicati e complessi. Vi sono timori, attese, fraintendimenti dalle due parti. I ragazzi si chiedono chi siano mai questi giovani poco più grandi di loro che entrano a scuola una o due volte la settimana e che li aiutano a capire e a studiare. Ma anche i giovani tutor, come possiamo leggere nei frammenti dei diari di bordo dei tutor milanesi riportati sopra, sono intimoriti e preoccupati di trovare un proprio ruolo tra gli insegnanti dei laboratori ai quali si affiancano e i ragazzi che devono accompagnare.

Tutor e tutorati, nella prima fase della reciproca conoscenza, stanno in punta di piedi, in attesa di segnali di domande e di primi tentativi di risposta. Piano piano l'imbarazzo si scioglie, i sorrisini nascosti diventano risate 
condivise, lo sguardo che sfugge trova una sosta, il linguaggio non verbale mostra meno chiusure e prefigura confini più porosi. Le strategie che $\mathrm{i}$ tutor adottano per "rompere il ghiaccio" sono soprattutto di due tipi. In alcuni casi, hanno a che fare con il racconto - seppure faticoso, date le competenze linguistiche ancora iniziali degli adolescenti stranieri, dei reciproci interessi extrascolastici. Che tipo di musica apprezzano gli uni e gli altri? Che sport hanno praticato e vorrebbero continuare a praticare in Italia? Quali erano i loro interessi nel Paese d'origine? In altri casi, sono le lingue ad unire e a gettare i primi ponti: le parole in lingua madre, o in un codice veicolare comune, creano immediatamente familiarità e legami, rompono solitudini pesanti fino ad allora suite e sopportate. Ed "è in quei momenti che i ragazzi hanno delle idee fantastiche", come scrive una tutor cinese a proposito dei ragazzi connazionali che ha seguito, che si sono "sbloccati" e aperti anche grazie alla sua presenza e alla possibilità di dirsi in lingua madre. L'eterogeneità nazionale e linguistica che ha contraddistinto il gruppo dei tutor si è rivelata essere ancora una volta un elemento cruciale e un'opportunità per tutti. Essa ha comunicato a tutti la "normalità" della diversità, l'importanza della storia di ciascuno, il valore di ogni lingua, la possibilità di essere per ciascuno, insieme e nello stesso tempo, uguale e diverso rispetto agli altri.

L'intervento dei tutor nel progetto "Almeno una stella" ha avuto una durata complessiva di circa 200 ore nei due anni: 80 ore durante il primo anno (preparazione all'esame di terza media) e 80 il secondo anno (aiuto allo studio in prima superiore), comprensive delle ore di formazione dei tutor e dedicate agli incontri in itinere. Alla luce dell'esperienza, possiamo scandire il loro impegno e l'accompagnamento educativo in quattro diverse fasi che possiamo definire così:

- la preparazione, l'avvio e l'assunzione del ruolo;

- l'accompagnamento educativo e la centralità del compito scolastico;

- l'approfondimento della relazione educativa e della conoscenza reciproca;

- la chiusura e il commiato.

\section{I fase. La formazione e l'assunzione del ruolo}

Durante la prima fase, si è formato il gruppo dei tutor. Essi sono stati individuati grazie ai contatti con le università e le scuole superiori, attraverso auto-candidature e in seguito a colloqui individuali. Subito dopo ha preso avvio il percorso formativo scandito in quattro incontri. I temi posti al centro del momento di formazione hanno riguardato la propria storia personale e scolastica; una prima conoscenza della situazione degli adolescenti stranieri e del loro inserimento a scuola; lo scambio e la prefigurazione rispetto al ruolo. 
Dopo gli incontri di formazione iniziali, i tutor hanno iniziato il loro viaggio educativo, attrezzati di qualche conoscenza in più e affollati ancora da molti dubbi sul che fare e come fare. Lincontro iniziale con i ragazzi ai quali sono stati abbinati ha segnato l'inizio dell'avventura professionale e umana. Come avvicinarsi a loro? Come conoscerli? Come passare messaggi di fiducia e di disponibilità? Nelle prime fasi, uno strumento utile per la reciproca conoscenza si è rivelato quello dell'autopresentazione. I tutor hanno raccontato alcuni aspetti della propria storia e i ragazzi si sono presentati sulla base di qualche domanda e alcuni sollecitatori (i tutor stessi hanno elaborato una traccia di intervista/tipo da rivolgere agli adolescenti dal titolo Come va la vita?).

Nei due anni di progetto, il gruppo dei tutor è stato inoltre seguito e accompagnato strada facendo durante incontri puntuali, a volte rivolti solo a loro, altre volte aperti anche agli insegnanti con i quali stavano collaborando. Sono stati questi momenti importanti per discutere insieme, rivedere il proprio ruolo, apprendere dall'esperienza degli altri, mettere in comune strumenti e modalità efficaci di relazione educativa con gli adolescenti.

\section{II fase. Abbiamo un compito; facciamo un patto}

La seconda fase del lavoro di tutoraggio ha posto al centro il compito scolastico e cognitivo al quale le attività di laboratorio cercavano di dare risposta. La priorità delle iniziative educative del primo anno consisteva nella preparazione dei ragazzi stranieri a sostenere l'esame di terza media oppure nell'accompagnamento iniziale nella scuola superiore. In questa fase, il traguardo era chiaro e ben delineato e il tutor ha funzionato soprattutto come una risorsa al servizio della scuola e dell'obiettivo comune. I tempi erano stretti, le energie dovevano essere convogliate in maniera efficace: il patto tra tutor e i ragazzi accompagnati era dunque molto chiaro. In questa fase, alcuni tutor si sono sentiti a volte troppo costretti e vincolati a compiti scolastici che lasciavano poche possibilità di comunicare con gli adolescenti stranieri in maniera più aperta, informale, autentica. Per altri invece, l'obiettivo comune e chiaramente definito ha contribuito a rendere maggiormente individuabile e più gestibile il proprio ruolo di tutor scolastico.

Che cosa hanno fatto i tutor in questa fase? Hanno sostenuto i ragazzi nello studio delle diverse discipline, li hanno aiutati nei compiti e ad affrontare prove e verifiche. Li hanno sorretti soprattutto nello sforzo, faticoso e cruciale, di trovare e sperimentare un proprio metodo di studio in una scuola diversa per organizzazione, richieste, impostazione didattica, rispetto a quella sperimentata nel Paese di origine.

I. così descrive una giornata/tipo di questa fase. 


\section{Ti mostro come si studia}

Io mi siedo, un po' a caso, accanto ad A. e H., che stanno raccogliendo informazioni sulla geografia e la storia del Marocco. A. e H. sembra si contendano la mia attenzione, le richieste mi vengono fatte contemporaneamente (riassumere, spiegare il significato di alcune parole, impostare la pagina ecc.). A. mi consulta per scegliere i colori ed il carattere del titolo, $\mathrm{H}$. dice di non saper andare avanti da sola; $\mathrm{H}$. è sicuramente più in difficoltà, si distrae facilmente e distrae anche A. che oggi non ha moltissima voglia di impegnarsi.

Cerco di orientarmi sul lavoro svolto da loro in precedenza e di programmare ciò che devono fare, stabilendo i tempi del mio intervento e spostando l'attenzione dall'una all'altra con ordine, comunicandolo a loro: le incoraggio e cerco di capire quali siano le reali difficoltà di ciascuna, per calibrare il mio intervento.

Non hanno libri di testo a disposizione, il materiale viene cercato su internet, la fonte privilegiata è wikipedia. Quella mattina, a scuola è stata distribuita una chiavetta USB, con la quale hanno giocato fino a pochi minuti prima, sulla quale salveranno di volta in volta il lavoro svolto. Mi riprometto di cercare a casa del materiale e di portarlo.

A. mi consulta per scegliere gli argomenti di cui parlare nella tesina.

Riguardo all'imparare a memoria, ai ragazzi, ho detto, in modo diverso, che capivo che la cosa li rendesse più sicuri, ma che sarebbe stato meglio che riuscissero a trovare, loro, le parole giuste; a P. precedentemente avevo avuto il tempo di mostrare come fare e il ragazzo ha poi dimostrato di essere perfettamente in grado di esprimere i concetti in modo corretto, usando i termini adeguati, sembrava molto soddisfatto. Ho anche detto loro che uno dei rischi dell'imparare a memoria è quello di dimenticare quando si è un po' emozionati e quindi di bloccarsi, ho aggiunto però che mi ero accorta che sapevano rispondere alle domande e che questo era molto importante, perché saper rispondere ad una domanda, sblocca.

Ho cercato anche di non farmi coinvolgere troppo dalle loro richieste, anche se è stato difficile in alcuni momenti, soprattutto negli ultimi incontri. (Dal diario di I.)

\section{III fase. La relazione si approfondisce}

Nelle situazioni più informali, sulla soglia, prima e dopo le attività scolastiche, già durante la seconda fase, la relazione fra i tutor e i ragazzi si fa via via più densa e vicina. E lentamente prende forma un terzo momento, durante il quale i tutor hanno mantenuto il loro ruolo di "bussola educativa", ma sono diventati nel frattempo anche un supporto, dei consiglieri, qualcuno di cui fidarsi e al quale porre domande, esprimere dubbi, confidare emozioni. L'aver condiviso la stessa esperienza di migrazione e di spaesamento e la fatica a ri-orientarsi ha avvicinato i più grandi agli adolescenti. Poter raccontarsi e dire di sé nella propria lingua ha permesso ad alcuni neoarrivati, fino a quel momento privi di parola e dunque privati della propria storia, di evocare l'altrove, il "prima" e la casa dalla quale si sono sentiti strappati senza averlo scelto. 
Prossimità di età, di genere, di lingua, di biografia: sono tante le comunanze che hanno reso con il tempo la relazione più densa e che collocano il ruolo del tutor in una terra di mezzo, al crocevia delle storie e degli spazi educativi e personali. Oltre ad accompagnare e sostenere l'apprendimento, il tutor in questa fase ha contribuito spesso a dare/far ritrovare la fiducia in se stessi a coloro che attraversavano momenti di scoraggiamento e che avevano perso la motivazione a provare e riprovare. Ha rappresentato per i ragazzi un esempio positivo proprio di chi ha vissuto le stesse difficoltà e ha sperimentato i medesimi fardelli e tuttavia è riuscito a farcela.

\section{IV fase. Mantenere dei fili}

Nella fase finale, il tutor si è preso il tempo per congedarsi dai ragazzi che ha seguito; si è posto e ha posto loro delle domande sull'efficacia del suo ruolo. I ragazzi hanno ora qualche strumento in più per procedere in maniera autonoma? Hanno capito almeno un po' come si studia e come ci si organizza? Alcuni di loro ritrovato la fiducia in se stessi e interiorizzato il messaggio che si può riuscire e che si deve continuare a provarci?

Il momento del distacco è stato attenuato dalla reciproca promessa di mantenere i contatti, di continuare a comunicare magari a distanza. Ed è successo infatti che i ragazzi seguiti abbiano contattato i loro tutor in una fase successiva per raccontare come andava la scuola, come riuscivano a cavarsela nello studio, quali voti avevano preso nella verifica... O anche solo per chiedere un'informazione, un consiglio, un orientamento, oppure semplicemente salutare e ridarsi appuntamento.

\section{Le quattro fasi del tutoraggio}

\begin{tabular}{|l|l|l|}
\hline Prima fase & $\begin{array}{l}\text { Formazione dei } \\
\text { tutor e avvio } \\
\text { dell'esperienza }\end{array}$ & $\begin{array}{l}\text { - I tutor seguono un percorso formativo iniziale di } \\
\text { circa } 20 \text { ore. } \\
\text { - Si provvede a formare i sottogruppi, eterogenei } \\
\text { per genere, età, nazionalità e lingua e a fare l'abbi- } \\
\text { namento con le diverse scuole e con i docenti. } \\
\text { - Nella fase di avvio, i tutor si presentano ai ragaz- } \\
\text { zi; chiariscono il loro ruolo e cercano di conoscere i } \\
\text { ragazzi che seguiranno. }\end{array}$ \\
\hline Seconda fase & $\begin{array}{l}\text { Centralità } \\
\text { del compito } \\
\text { scolastico e del } \\
\text { patto formativo } \\
\text { come facilitatori di apprendimento (tutor scolastico). } \\
\text { - La centralità è occupata dal compito da superare } \\
\text { e dall'obiettivo da raggiungere (ad esempio, prepa- } \\
\text { rarsi a superare l'esame di terza media). } \\
\text { - Fra i tutor e i ragazzi seguiti si stabilisce un patto } \\
\text { formativo esplicito e che riguarda il compito. } \\
\text { - In questa fase, i tutor aiutano i ragazzi a studiare, } \\
\text { ricercare, affinare capacità e competenze. } \\
\text { - E cercando di aiutarli anche a organizzarsi e a } \\
\text { trovare un proprio metodo di studio, rifacendosi } \\
\text { anche alla propria esperienza di alunno. }\end{array}$ \\
\hline
\end{tabular}




\begin{tabular}{|l|l|l|}
\hline Terza fase & $\begin{array}{l}\text { L'approfondirsi } \\
\text { della relazione } \\
\text { e dello scambio }\end{array}$ & $\begin{array}{l}\text { - In una fase successiva, via via che la conoscenza } \\
\text { si intensifica, i ragazzi stabiliscono con il tutor una } \\
\text { relazione di apertura e fiducia. } \\
\text { - Pongono domande e cercano risposte che hanno } \\
\text { a che fare con la vita di ogni giorno, le amicizie e gli } \\
\text { affetti, i progetti per il futuro... } \\
\text { - Esprimono i loro disorientamenti e timori, cerca- } \\
\text { no conferme e consigli. Il ruolo del tutor si fa più } \\
\text { delicato e complesso, ma può rivelarsi efficace per } \\
\text { rassicurare, rimotivare, ridare fiducia in se stessi. } \\
\text { In questo caso, oltre ad essere un "tutor scolasti- } \\
\text { co", il giovane, italiano o straniero, può rappresen- } \\
\text { tare per gli adolescenti un esempio e un "modello" } \\
\text { di identificazione positiva. }\end{array}$ \\
\hline Quarta fase & $\begin{array}{l}\text { La chiusura } \\
\text { e il commiato } \\
\text { (non definitivo) }\end{array}$ & $\begin{array}{l}\text { gli adolescentine dell'intervento di tutoraggio, i tutor e } \\
\text { ragazzi sono più autonomi? Che cosa hanno impa- } \\
\text { rato? Credono un po' di più in se stessi? } \\
\text { - E i tutor che cosa hanno imparato da questa } \\
\text { esperienza? } \\
\text { - Oltre che il momento per raccontare e ripercorre- } \\
\text { re il cammino fatto insieme dai due punti di vista, il } \\
\text { commiato è anche l'occasione per darsi appunta- } \\
\text { mento, mantenendo dei fili e la possibilità di risen- } \\
\text { tirsi. }\end{array}$ \\
\hline
\end{tabular}

\section{La bellezza della responsabilità}

Alla fine dell'esperienza di tutoraggio, soprattutto coloro che hanno potuto svolgere questo ruolo in maniera continuativa e per due anni hanno espresso una valutazione fortemente positiva. Innanzi tutto, l'esperienza ha consentito loro di allargare gli orizzonti, di lavorare con altri coetanei per stare al fianco di ragazzi più piccoli con storie di migrazione e di spaesamento da accogliere e gestire con cura. Il loro ruolo si è inoltre modificato e articolato nel tempo: inizialmente, si trattava di sostenere i minori stranieri nel compito dello studio o nella preparazione all'esame. Ma fin da subito i tutor hanno capito che le implicazioni relazionali e affettive erano fortemente intrecciate e si sono assunti il ruolo di compagni di viaggio un po' più esperti, di fratelli e sorelle maggiori, protettivi ed incalzanti, di informatori e guide nell'esplorazione dei nuovi territori e degli spazi potenzialmente comuni.

Il tema della giusta distanza è tornato di frequente negli incontri con $\mathrm{i}$ tutor, sia nella fase iniziale della loro formazione, sia nei momenti in itinere dedicati alla supervisione e allo scambio. Prossimi, ma non totalmente vicini; compagni di strada ma non del tutto pari; simili nell'esperienza vissuta ma non identici: questo tema e le sue sfumature e implicazioni è stato spesso al centro delle riflessioni di gruppo e del confronto fra i tutor. 
Questa esperienza mi ha fatto sentire veramente una stella sul cammino dei ragazzi che ho seguito. Dalle loro preoccupazioni e bisogni ho provato a risolverli. Ero sempre disponibile per loro, anche loro sono state le mie 'stelline'. C'era una solidarietà tra di noi. (Dal diario di J.G.)

Osservando dall'esterno le esperienze che gli altri tutor hanno avuto con i ragazzi e vivendo esperienze simili in prima persona, mi sento di affermare che in alcuni casi il tutor diventa un amico, un fratello, un confidente, una guida, un sostegno, dentro e fuori la scuola. Molti ragazzi danno al proprio tutor l'opportunità di far parte del loro mondo, quello che un tutor può fare è cogliere questa preziosa occasione e non tirarsi indietro. (Dal diario di E.D.M.)

Noi tutor avevamo un compito importantissimo: non si trattava soltanto di aiutare i ragazzi ad imparare l'italiano o a fare bene i compiti, ma soprattutto di infondere fiducia nei ragazzi verso la loro nuova realtà, di aiutarli a non avere paura di ciò che li aspettava qui e di cercare di tirarli fuori dal guscio dentro cui inevitabilmente si erano nascosti per proteggersi.

In questo lungo cammino iniziato più di un anno fa, ho potuto osservare la crescita e la maturazione dei ragazzi che ho seguito: all'inizio erano timidi, taciturni, non dicevano una parola in italiano neanche ad obbligarli. Ora sono disinvolti, temerari e sembra che abbiano trovato il loro posto nella loro nuova vita. (Dalla relazione di A.D.)

Ho potuto vedere una realtà di giovani stranieri che sta formando una nuova Italia, giovani competenti e con voglia di fare, molto diversi da quelli che vogliono farci credere essere i giovani d'oggi, soprattutto se stranieri, i giornali o la tv. Sono grata al progetto "Almeno una stella" per avermi permesso di conoscere così tante persone positive e vorrei che tutti avessero l'opportunità di vedere ciò che ho potuto vedere io passando del tempo con questi fantastici ragazzi. (Dalla relazione di B.B.)

\section{I diversi volti del tutoraggio}

La varietà delle azioni condotte nei diversi territori nell'ambito del progetto "Almeno una stella", pur nella condivisione degli obiettivi centrali, ha permesso di mettere in luce e sostanziare la potenzialità del dispositivo e la ricchezza insita nel cammino di tutoraggio. I giovani studenti, universitari o di scuola superiore, che hanno assunto il ruolo di "stelle" sul cammino degli adolescenti hanno potuto sperimentare i diversi modi di accompagnare e di stare accanto.

Proviamo a delinearli, così come essi traspaiono anche dai diari di bordo.

Vi è innanzitutto il tutor di compito.

È l'accompagnatore che aiuta i ragazzi a superare una prova di tipo scolastico, sia essa la preparazione all'esame di terza media, la comprensione 
di un testo di studio o la traduzione di un testo scritto. Il tutor di compito affianca il docente di laboratorio, agisce a volte in maniera autonoma, scambia le proprie competenze specifiche con quelle degli altri tutor, mettendole a disposizione del gruppo. Il tutor di compito si assume anche il ruolo di gestire i tempi, sollecitare la puntualità, chiedere e sollecitare risposte e sforzi individuali.

Vi è poi il tutor di relazioni. Sono il fratello o la sorella maggiore che tessono legami; sono attenti alla storia di ciascuno; aprono dei varchi nelle chiusure iniziali, costruiscono occasioni di scambio e di ulteriore appuntamento narrativo. Questo ruolo, cruciale nel progetto "Almeno una stella", richiede attenzioni, pazienza, tempi lunghi. Richiede che piano piano si creino consuetudini e appuntamenti, che si manifestino legami e simpatie, che si abbassino difese e barriere. Nella prima fase, in genere, tutor e tutorati sono concentrati sul compito e sulla sfida da superare. La relazione di vicinanza si costruisce negli interstizi fra un dovere e l'altro, nei momenti informali prima o dopo i laboratori, quando le difese si allentano e la fiducia reciproca lascia il posto all'imbarazzo, al silenzio e al timore di "perdere la faccia".

I giovani accompagnatori diventano anche tutor di cittadinanza. In questa veste, fanno da guida ai ragazzi stranieri nella scoperta e nell'esplorazione della città e dei quartieri. In molti casi, si è osservato che gli adolescenti di recente immigrazione vivevano una condizione di solitudine e di isolamento nel tempo extrascolastico. Nel momento della vita in cui, in genere, i minori allargano il loro spazio vitale al di fuori della dimora, può succedere invece che la migrazione blocchi questo viaggio e restringa $i$ loro spazi in maniera significativa. I ragazzi stranieri inoltre poco conoscono la città e i luoghi comuni in cui vivono da tempo. A questo proposito, un questionario proposto agli adolescenti coinvolti nel progetto a Torino ha evidenziato le scarse informazioni e l'uso ridotto dei luoghi pubblici e delle opportunità di aggregazione aperte a tutti.

I tutor possono aprire nuovi orizzonti, orientare e informare, organizzare visite guidate e incontri significativi con enti e associazioni del territorio. Possono sollecitare i ragazzi a partecipare a iniziative artistiche ed espressive per valorizzare talenti, promuovere lo scambio interculturale, moltiplicare i linguaggi. Un esempio concreto può essere individuato nell'organizzazione da parte dei tutor della partecipazione degli adolescenti stranieri alla Giornata internazionale della Lingua Madre, durante la quale essi hanno potuto leggere, raccontare ed esprimersi nel loro codice materno.

Tutti i tutor hanno inoltre rivestito un ruolo importante per permettere ai ragazzi seguiti di diventare un po' più resilienti. Il tema della resilienza è centrale nel progetto. Come abbiamo scritto, il titolo stesso fa riferimento agli studi e alle ricerche di B. Cyrulnik sulle situazioni di vulnerabilità dei minori e sulla necessità di poter contare su "tutori di resilienza". La pre- 
senza stessa dei tutor sul cammino di inserimento e di ri-orientamento nella nuova scuola e nella nuova realtà è stata per molti adolescenti un fattore positivo e di protezione.

Il tutor rappresenta infatti una figura nella quale identificarsi, un modello positivo al quale fare riferimento, uno stimolo costante a dirsi "ce la posso fare anch'io". Il tutor è una spalla, un'ancora, un punto di riferimento e di ascolto, un amico e un fratello. Ma perché questo legame si crei e si rinsaldi, ci vuole del tempo, come i frammenti dei diari dei tutor trentini sottolineano.

Il trovarsi a fianco di E., non come insegnante o amica, ma come persona disposta ad ascoltare, è stata, credo, l'essenza del percorso compiuto. (Dal diario di bordo di A.)

Alla fine di quest'esperienza di due anni con I. e T., posso dire che si è creato con le ragazze un forte legame. Prima di quest'anno I. non si era mai lasciata abbracciare, mentre oggi ci salutiamo come due amiche. (Dal diario di bordo di C.)

Certo, non è sempre stato un percorso agevole: conquistare la fiducia, e in certi casi abbattere la diffidenza, di ragazzi che non sempre avevano scelto il percorso consapevolmente; ci è voluto del tempo... (Dal diario di bordo di T.)

A. si è dimostrato, incontro dopo incontro, sempre più aperto. Le prime volte era taciturno; pensavo fosse un problema linguistico, in realtà era necessario del tempo perché si creasse un rapporto di fiducia... (Dal diario di bordo di G.)

Il legame che si è creato con le ragazze va ben oltre l'aiuto nello studio: si è creato un legame di affetto fraterno. Anche se il progetto è finito e le nostre vite prenderanno strade diverse, ci siamo ripromesse di tenerci in contatto con messaggi e telefonate e di vederci ogni tanto. (Dal diario di bordo di C.)

Mi dispiace di non essere riuscita del tutto a far capire alle ragazze l'importanza della scuola e dell'impegno, anche nella vita, della costanza e dell'essere sempre presenti e puntuali. (Dal diario di bordo di C.)

L'incontro con loro mi ha sempre profondamente arricchito: lo scambio di storie, l'ascolto dei loro vissuti, spesso difficili e dolorosi, mi hanno donato più di quanto io potessi offrire loro in termini d'insegnamento. La possibilità che mi è stata data dal progetto "Almeno una stella" è stata, per me, estremamente preziosa. M'incuriosiva l'adozione dello strumento del tutoring nella costruzione di un rapporto educativo in ambiente scolastico e sociale. Inoltre costruire questo percorso assieme a un adolescente, in una fase della vita delicata in cui si snodano la costruzione dell'identità e la scoperta del sé, ha rappresentato una bella sfida educativa, anche dal punto di vista professionale. (Dal diario di bordo di A.) 
Dopo quest'esperienza, forse i tutor diventeranno educatori, mediatori e insegnanti più attenti ed efficaci (coloro che seguono questo tipo di studi). Ma tutti certamente guarderanno i ragazzi della migrazione in maniera diversa: con maggior coinvolgimento e con una forte dose di empatia.

\section{Riferimenti bibliografici}

Beneduce R. (1988), Frontiere dell'identità e della memoria, FrancoAngeli, Milano.

Commissione delle Comunità Europee (2008), Libro Verde. Migrazione e mobilità: le sfide e le opportunità per $i$ sistemi d'istruzione europei, Bruxelles.

Cyrulnik B. (2009), Autobiografia di uno spaventapasseri, Raffaello Cortina, Milano.

Cyrulnik B., Malaguti E. (2005), Costruire la resilienza, Erickson, Trento.

Eurydice (2004 e 2009), Integrating Immigrant Children into Schools in Europe, Commissione Europea, Bruxelles.

Favaro G. (a cura di) (2013), Bussole sul cammino. Un progetto di accoglienza scolastica per gli adolescenti stranieri, Centro COME, Milano.

Malaguti E. (2005), Educarsi alla resilienza, Erickson, Trento. 


\section{Allegato 1}

\section{II "diario di bordo" di S.}

\section{Mi presento}

\section{Auto-presentazione}

Mi chiamo S., ho 22 anni e sono un'immigrata di seconda generazione. I miei genitori sono entrambi di origine egiziana, io e i miei due fratelli invece siamo nati e cresciuti a Milano e abbiamo seguito il nostro percorso scolastico interamente in Italia. Personalmente, mi sento quasi totalmente italiana, non solo perché posseggo la cittadinanza, ma anche perché la mia vita ormai I'ho vissuta interamente in Italia; infatti quando vado in Egitto, durante le vacanze estive per far visita ai miei parenti, mi sento come se fossi una turista in un paese tuttavia famigliare. Eppure mi sento appartenente anche alla cultura araba: la tradizione araba la respiro tutti i giorni in casa parlando in arabo con i miei, guardando la tv satellitare che trasmette programmi in arabo, mangiando piatti tradizionali sia italiani che egiziani, e professando la religione islamica.

Mi sono diplomata alla scuola "G. Cardano" di Milano con indirizzo in PACLE (perito aziendale corrispondente in lingue estere) e sono laureanda al corso di studi di "Comunicazione interculturale" in Bicocca.

Ho mirato la mia scelta formativa nel campo delle lingue straniere perché credo che la lingua sia un ottimo mezzo per conoscere le diverse culture, inoltre capisco quale tipo di difficoltà linguistiche possono avere i ragazzi stranieri nelle scuole italiane, data la mia esperienza anche come insegnante di arabo a bambini di origine arabofona nella scuola "Dolci" nel doposcuola. Per questi motivi, mi piacerebbe proseguire i miei studi nel campo della logopedia, in modo da aiutare le persone che hanno difficoltà linguistiche di ogni genere.

\section{Che cosa penso dei ragazzi e delle ragazze immigrati}

Essendo io stessa figlia di immigrati provenienti dall'Egitto, credo che i ragazzi e le ragazze immigrati siano persone molto forti e coraggiose, che non scelgono di lasciare il proprio Paese per capriccio, ma per varie necessità o motivi di tipo economico, sociale o politico. Lasciare il proprio paese di origine per queste persone significa lasciare tutto ciò che conoscono e hanno di famigliare, per avventurarsi in un paese spesso sconosciuto con la speranza di un futuro migliore.

Gli immigrati hanno bisogno di sentirsi ben accolti dal paese che li ospita, perché spesso scappano da una situazione ostile e non credo abbiano bisogno di incontrare nuovi ostacoli, soprattutto in un paese nuovo ai loro occhi dove non sanno ancora come orientarsi.

Penso che sia fondamentale aiutare gli immigrati, soprattutto i neo-arrivati, in modo da poter dar loro il modo di muoversi in maniera indipendente nel territorio e di superare i vari ostacoli che incontreranno; credo che questo sia 
il primo passo verso l'integrazione, cercando comunque di far conservare a ciascuno la propria identità culturale.

Penso che gli immigrati portino insieme a loro delle importanti risorse sociali come la loro cultura e la loro storia, le quali hanno il potere di contribuire a una visione più ampia del mondo che ci circonda.

\section{Come immagino il mio ruolo da tutor}

Immagino il mio ruolo da tutor come una figura d'aiuto allo studio ma anche come una sorta di "sorella maggiore" pronta a dare buoni consigli utili su come affrontare al meglio l'esame di terza media ed altri problemi presenti nella vita quotidiana. Spero di riuscire a fornire loro un buon metodo di studio che li possa servire a continuare al meglio gli studi nel sistema scolastico italiano. Inoltre spero anche di instaurare un buon rapporto di fiducia con questi ragazzi, con la consapevolezza che stanno attraversando questo delicato periodo della loro vita, cioè l'adolescenza, in un paese in cui non sono ancora abituati.

\section{Diario dell'esperienza}

\section{I anno - Nella scuola secondaria di primo grado}

\section{Prima osservazione. Data: 25/02/14}

\section{Descrivo la situazione}

È il mio primo giorno da tutor alla scuola Ciresola, in zona Loreto, faccio conoscenza della coordinatrice e degli altri tutor che collaboreranno con me, e scopro con stupore che questi ultimi frequentano la mia stessa università tranne uno. Noi tutor entriamo in un'aula al secondo piano, e qui incontro per la prima volta i ragazzi: per conoscerci meglio, disponiamo le sedie presenti in classe in cerchio in modo che tutti possano guardarsi e conoscerci. A turno, ognuno di noi, sia i ragazzi che i tutor, ci presentiamo parlando delle nostre origini, della nostra storia e dove ci piacerebbe viaggiare.

\section{Le richieste, i bisogni, gli atteggiamenti dei ragazzi}

C'è un atteggiamento quasi distaccato tra gli studenti e i tutor, ma è comprensibile visto che è la prima volta che ci incontriamo. Alcuni ragazzi descrivono il loro paese di origine alcuni col desiderio di tornarci al più presto, mentre altri sono meno desiderosi di ritornarci. Si intuisce che il bisogno generale dei ragazzi sia quello di conoscere meglio l'Italia e orientarsi meglio nella città in cui vivono perché sono ancora molto legati alle radici del loro paese di origine.

\section{Il mio intervento}

II mio intervento di oggi è stato quello di presentarmi raccontando le mie origini e i miei studi, in modo da creare una sorta di vicinanza con i ragaz$\mathrm{zi}$, soprattutto quelli di origine araba. A fine lezione poi mi sono state affidate due ragazze arabe, $\mathrm{S}$. dal Marocco e $\mathrm{D}$. dalla Siria, a cui ho spiegato in che modo le aiuterò a prepararsi per l'esame di terza media, cioè facendo eser- 
cizio sul tema di italiano, concentrandoci sulla lettera o il diario e preparando insieme il power point per la tesina.

\section{Seconda osservazione. Data: 08/04/14}

\section{Descrivo la situazione}

Siccome oggi c'era una bella giornata, siamo andati in cortile a giocare un po', in modo da creare una collaborazione e complicità tra noi tutor e i ragazzi. Di solito le ragazze e noi tutor giochiamo ai passaggi di pallavolo mentre alcuni dei ragazzi giocano a basket, ma oggi si sono uniti a noi J. P. e E., due dei ragazzi della nostra classe. Oggi è stato il primo giorno in cui ho lavorato con E.: non ha frequentato molto il nostro corso prima di oggi, e precedentemente aveva lavorato con un'altra tutor ma oggi si è unito alle due ragazze che mi sono state affidate. La relazione che ho con le altre due ragazze si è consolidata, tanto che durante le pause tra un esercizio e l'altro ampliamo la nostra conoscenza raccontandoci della Siria, del Marocco e dell'Egitto in confronto con l'Italia. Con E. invece c'è stata poca confidenza all'inizio, ma quando ha scoperto che pure io ero originaria dall'Egitto, si è subito sentito a suo agio parlandomi anche in arabo.

Le richieste, i bisogni, gli atteggiamenti dei ragazzi

Il tema dell'esercizio sul diario di oggi è stato "racconta una tua triste esperienza": grazie a questo tema ho potuto conoscere meglio le paure e ciò che fa rabbia ai ragazzi e sono emersi degli aspetti molto interessanti. Hanno cercato di scrivere molto e in maniera corretta, cercando aiuto nell'esprimere in italiano i loro stati d'animo. Le due ragazze hanno raccontato un episodio che riguardava per entrambe la loro difficoltà linguistica, infatti $D$. ha raccontato di una volta in cui è andata a fare compere e si è sentita a disagio e nel panico quando non ha capito cosa le diceva la cassiera; mentre S. ha raccontato di un episodio avvenuto a scuola in cui ha cercato di fare del suo meglio in un'interrogazione, ma ha preso comunque un brutto voto. Per quanto riguarda $\mathrm{E}$., è emerso che ha un temperamento violento con alcuni dei suoi compagni di scuola, ma solo quando viene provocato. Si è comunque sentito a suo agio nel raccontarmi la sua esperienza di litigio coi suoi compagni, è stato un modo di conoscerlo meglio e quando gli ho dato dei consigli su come comportarsi si è instaurata una sorta di fiducia.

\section{II mio intervento}

II mio intervento di oggi è stato far capire bene la traccia e dare degli esempi prima che iniziassero a scrivere, correggere e infine commentare insieme a loro ciò che hanno scritto. Nel complesso sono stati molto bravi e si sono lasciati coinvolgere dalla trama scrivendo più di quanto avessero scritto negli esercizi precedenti. Siccome E. ha raccontato il fatto che ha litigato con dei suoi compagni di scuola ma che voleva coinvolgere altra gente più grande al di fuori della scuola per dare una "lezione" a questi ragazzi, gli ho suggerito vivamente di evitare i guai e di lasciare perdere; è stato come se parlassi a mio fratello minore, e spero che lui abbia seguito il mio suggerimento. 


\section{Terza osservazione. Data: 29/04/14}

\section{Descrivo la situazione}

Dopo aver giocato un po' in cortile con tutti i ragazzi, oggi è stato il primo giorno in cui ci siamo trovati al laboratorio di informatica, per incominciare la stesura della tesina in power point. Ormai il clima col mio gruppo si è consolidato, riusciamo a lavorare insieme in armonia e le ore di studio non sembrano passare lentamente, anzi spesso i ragazzi mi raccontano le loro giornate, i loro timori riguardo l'esame chiedendomi anche di aiutarli nelle altre materie quando abbiamo del tempo a disposizione.

Le richieste, i bisogni, gli atteggiamenti dei ragazzi

Con mia sorpresa, ho scoperto che in realtà i ragazzi avevano già iniziato la stesura senza il mio aiuto, e la loro richiesta è stata quella di completarlo insieme. Ognuno dei ragazzi che seguo si è postato davanti a un computer e, dopo aver inserito la chiavetta, a turno ho guardato il lavoro che avevano già iniziato. Hanno avuto un atteggiamento positivo nei miei confronti, ansiosi di farmi vedere cosa erano riusciti a fare da soli per presentare al meglio il loro paese di origine.

\section{II mio intervento}

II mio intervento è stato quello di seguire a turno D., S. e E. cercando di dividere il nostro tempo a disposizione in maniera equa. Ad ognuno di loro ho corretto le parti che non andavano bene della presentazione, successivamente ho cercato insieme a loro di creare un piano di lavoro su ciò che andava ancora fatto: facendo una mappa concettuale abbiamo cercato degli argomenti da trattare per ogni materia ma che siano tutti collegati al loro paese di origine. Attraverso questa mappa, che poi hanno inserito nella loro presentazione virtuale, siamo riusciti a capire cosa andava ancora fatto e in che modo poteva essere presentato, per esempio cercando informazioni su Wikipedia o inserendo foto prese da internet o dal loro archivio personale. Ho notato inoltre che sembravano molto più coinvolti nel lavoro al computer rispetto agli esercizi sulla lettera o sul diario perché hanno potuto esprimere la loro creatività nella presentazione.

\section{Quarta osservazione. Data: 13/06/14}

\section{Descrivo la situazione}

Oggi è la vigilia della prova scritta: è ormai la quinta volta che noi tutor ci troviamo da soli con i nostri ragazzi e, come da consuetudine ormai, ci siamo trovati tutti in cortile, solo che questa volta ci siamo rimasti poco per via del brutto tempo e per non togliere del tempo prezioso al ripasso. Erano presenti tutti e tre i ragazzi che seguo, anche perché il giorno prima mi avevano scritto un messaggio per sapere l'ora dell'incontro e se avremmo fatto un ripasso per la prova di italiano, così ci siamo diretti in aula computer e questa volta io e i miei ragazzi ci siamo riuniti in un unico tavolo per fare il ripasso insieme. La coesione del gruppo è diventata più consolidata, e i tre ragazzi quando trovavano delle difficoltà di tipo linguistiche, prima di chiedere il mio aiuto si consultavano, e se ne erano in grado, si aiutavano. 
Le richieste, i bisogni, gli atteggiamenti dei ragazzi

Le richieste dei miei ragazzi di oggi sono state quelle di concentrarci sulla prova di italiano e di mettere da parte per una volta il completamento della presentazione della tesina. Sia E., che D. e S. sembravano preoccupati dalla prova di italiano di domani, ma li ho rasserenati in quanto tutti e tre hanno fatto dei progressi a livello linguistico, e sicuramente sanno esprimersi meglio in italiano ora rispetto all'inizio del nostro laboratorio. Tuttavia questo loro timore che avevano della prova di domani li ha fatti lavorare con maggiore attenzione agli errori che commettono di solito (come la punteggiatura, le doppie ecc.), in modo da non commetterli durante l'esame.

\section{II mio intervento}

II mio intervento è stato di consegnare degli esercizi con alcune tracce per eseguire la lettera o il diario. Prima di iniziare a scrivere, ho spiegato ai ragazzi che durante la prova d'esame avevano delle tracce da scegliere, e in che modo potevano argomentarle per creare un discorso ben definito, per esempio facendo su un foglio di brutta uno schema da seguire per toccare ogni aspetto che si vuole trattare all'esame. Abbiamo prima ripassato la struttura della lettera e del diario (e con sorpresa ho notato che le ricordavano perfettamente), poi ognuno ha scelto la propria traccia e dopo averla commentata e argomentata insieme oralmente l'hanno stesa per iscritto. A fine lezione ho corretto insieme a loro le tre lettere (tutti e tre hanno scelto di fare la lettera) e l'esito è stato complessivamente buono, perché tutti e tre hanno fatto pochi errori e hanno argomentato bene la traccia.

\section{Quinta osservazione. Data: 26/06/14}

\section{Descrivo la situazione}

Oggi è il giorno dell'esame orale di D. e S.: entrambe oggi si sono presentate davanti alla commissione d'esame per mostrare il loro lavoro sulla Siria e sul Marocco. La prima è stata $\mathrm{D}$. e siccome aveva l'esame alle 11 del mattino, mi sono trovata con lei un'ora prima per ripassare e per poterle dare un sostegno morale. Ci siamo trovate in aula di informatica per poter fare gli ultimi ritocchi alla sua presentazione, e successivamente abbiamo fatto una piccola simulazione d'esame, nella quale è andata bene nonostante l'agitazione.

Poi di pomeriggio è stato il turno di S. e anche con lei mi sono trovata prima in aula informatica per poter ripassare la presentazione. Anche lei era molto agitata ma aveva studiato bene la sua presentazione, così, dopo aver fatto anche con lei una prova dell'esame orale, ho passato il tempo che avevo a cercare di tranquillizzarla e di sostenerla.

Le richieste, i bisogni, gli atteggiamenti dei ragazzi

Entrambe le ragazze avevano bisogno di un sostegno di tipo morale piuttosto che didattico, perché erano sicure e soddisfatte del lavoro che abbiamo fatto insieme durante il laboratorio, ma erano meno sicure su come poter esporre bene il loro paese attraverso il power point. Dopo averle convinte che l'esame non era nulla di impossibile e che avevano entrambe le capacità di poter fare una buona presentazione hanno acquistato più fiducia in loro 
stesse. Nell'attesa di essere chiamate dalle insegnanti per poter essere interrogate, le ragazze mi hanno dimostrato i loro timori di fare un discorso fluido in italiano, ma mi hanno anche mostrato di essere felici per il fatto che ero lì con loro in un momento così importante. Una volta finito l'esame mi hanno pure ringraziata per il lavoro che abbiamo svolto insieme, cosa che mi ha donato una gioia immensa.

II mio intervento

II mio intervento di oggi è stato quello di ripassare insieme davanti al computer la loro tesina e di costruire insieme a loro un discorso per parlare sia della Siria che del Marocco. Successivamente abbiamo aspettato insieme il loro turno d'esame chiacchierando sui nostri piani futuri (per esempio che lavoro vorrebbero fare, quali sono i loro programmi dell'estate, come affronteremo il mese di Ramadan qua in Italia...) davanti a un bicchiere di cioccolata. Durante l'esame sono entrata in aula con loro, e quando si sentivano in qualche modo in difficoltà mi guardavano per riprendere un po' di fiducia. Per entrambe l'esame è andato bene, sono riuscite a fare un buon discorso senza troppi intoppi, e per quanto riguarda D. le insegnanti erano molto soddisfatte perché non l'avevano mai sentita parlare così tanto.

\section{II anno - Nella scuola secondaria di secondo grado}

\section{Prima osservazione. Data: 20/01/2015}

\section{Descrivo la situazione}

Oggi è il nostro decimo incontro all'istituto professionale "Bertarelli", ma sorprendentemente dall'inizio del nostro laboratorio l'aula non è mai stata così piena: oltre ai soliti volti ormai noti, ci sono molti ragazzi nuovi che noi tutor ancora non conosciamo e sono soprattutto ragazzi che frequentano la scuola. La professoressa di italiano ha esortato alcuni dei suoi studenti a frequentare il laboratorio per avere un maggiore aiuto allo studio, ma nessuno di noi si aspettava una tale affluenza! Così oggi è stato come se fosse il nostro primo giorno di laboratorio dedicando buona parte del nostro tempo alla presentazione e alla conoscenza dei ragazzi, e ogni tutor ha creato un gruppo di lavoro a seconda delle materie di studio dei ragazzi presenti.

Le richieste, i bisogni e gli atteggiamenti dei ragazzi

I ragazzi con cui ho lavorato parevano diffidenti all'inizio, poco entusiasti di doversi fermare a studiare a scuola anche al pomeriggio. Tuttavia hanno scoperto che questo non è un laboratorio di italiano come aveva riferito a loro la professoressa di italiano, ma bensì un aiuto allo studio nelle materie in cui hanno maggiori difficoltà. Così dopo breve tempo, ho iniziato subito a lavorare coi ragazzi scoprendo anche un certo entusiasmo da parte loro di studiare e finire i loro compiti in maniera più facile e più efficace dello studio individuale a casa.

\section{II mio intervento}

Oggi ho mirato soprattutto a instaurare un rapporto di fiducia coi ragazzi per "rompere il ghiaccio" che si crea al primo incontro. Ho mostrato loro che 
si può studiare in maniera più facilitata usando parole più semplici del testo di storia che dovevano studiare. Uno dei ragazzi alla fine dell'incontro mi ha anche confidato che non credeva che questo laboratorio fosse così utile e divertente per lui e che verrà ai prossimi incontri volentieri, e ciò mi ha fatto molto piacere.

\section{Seconda osservazione. Data: 24/03/2015}

Descrivo la situazione

Oggi è stato l'ultimo incontro al "Bertarelli"; ho iniziato questo laboratorio a metà degli incontri esattamente il 09 febbraio. Non avrei mai creduto di affezionarmi tanto ai ragazzi di questo laboratorio in così breve tempo, anche perché essendo stata l'ultima tutor arrivata temevo di non riuscire a legare con i ragazzi tanto quanto gli altri tutor del laboratorio. Invece mi sbagliavo: abbiamo passato l'ultimo giorno festeggiando insieme e facendo dei giochi di gruppo in cui ognuno di noi doveva raccontare delle esperienze passate legate a determinati elementi come il fuoco, l'origine del proprio nome o favole legate all'infanzia, ed è stato un ottimo modo di avvicinare ancora di più le distanze tra tutor e tutorati. Concludere il laboratorio in questo modo è stato magnifico perché ha fatto sentire ogni partecipante del laboratorio parte integrante del gruppo.

Le richieste, i bisogni, gli atteggiamenti dei ragazzi

Alcuni dei ragazzi erano ancora un po' timidi e alcuni si vergognavano anche di parlare troppo in italiano perché temevano di commettere degli errori, ma vedendo che sia i tutor che altri ragazzi raccontavano con disinvoltura dei propri episodi dell'infanzia, hanno preso subito confidenza e partecipato al gioco di gruppo. Molti hanno fatto grandi progressi nella produzione orale in italiano, altri invece si sono aiutati aggiungendo alcune parole in inglese per farsi capire, ma tutti hanno partecipato con entusiasmo e si sono sentiti a loro agio.

\section{II mio intervento}

Dato che oggi non ho dovuto aiutare i ragazzi a studiare o a fare i compiti, mi sono dedicata a conoscere meglio i ragazzi e ho scoperto alcune loro paure e curiosità riguardo al loro futuro. Alcune delle ragazze mi hanno confidato che non avevano molta fiducia nelle loro capacità linguistiche e che temevano di non riuscire mai a imparare bene l'italiano, ma frequentando il laboratorio hanno capito che le difficoltà si presentano soprattutto all'inizio della scuola e più si impegnano, più aumentano le loro abilità linguistiche. Chiedendomi poi anche il mio percorso di studi dato che anch'io sono di origine straniera, molte di loro mi hanno detto che vorranno frequentare l'università per diventare veterinarie o avvocatesse e ciò mi ha fatto davvero piacere perché ho dato loro maggiore fiducia in loro stesse. 


\section{Terza osservazione. Data: 21/05/2015}

\section{Descrivo la situazione}

Oggi è il mio ultimo giorno di laboratorio all'istituto "Maxwell" e noi tutor ci siamo organizzati per portare delle merende da mangiare in compagnia per salutarci. Abbiamo comunque dedicato del tempo all'aiuto allo studio di alcuni ragazzi dato che sono le ultime settimane per recuperare le materie insufficienti, ma l'ultima mezz'ora l'abbiamo completamente dedicata ai festeggiamenti. Alcuni dei ragazzi erano molto tristi all'idea che fosse il nostro ultimo incontro e che il laboratorio non ci sarà più l'anno prossimo.

Hanno confidato a noi tutor che si sono trovati bene con noi e che ormai venivano volentieri agli incontri, non solo perché lo studio era facilitato ma anche perché era divertente.

Le richieste, i bisogni, gli atteggiamenti dei ragazzi

Molti dei ragazzi hanno chiesto di rinnovare il laboratorio l'anno prossimo, e se questo non fosse possibile, almeno di rimanere in contatto coi tutor. II clima di festa ha reso questa giornata un po' meno triste per tutti i presenti, soprattutto per noi tutor che ormai ci siamo affezionati a questi ragazzi.

\section{II mio intervento}

Prima della merenda collettiva ho seguito A. nello studio e ho notato un miglioramento in lei: mi ha chiesto di studiare insieme diritto ma stavolta, dopo aver letto insieme il testo, riusciva a ripetermelo con parole sue anche senza il mio aiuto. Mi ha confessato ormai che il è diventato il suo metodo di studio leggere e riassumere i testi didattici con parole sue più semplici per capirlo e memorizzarlo meglio, proprio come avevamo fatto negli incontri passati. Prima di salutarci poi, avviandoci verso la metro, mi ha ringraziata di tutto l'aiuto dato durante i nostri incontri e mi ha chiesto se potevamo comunque sentirci tramite telefono anche se il laboratorio è finito, e ciò mi ha reso molto felice.

\section{Ritratti: le ragazze e i ragazzi che ho accompagnato}

D.: è una ragazza di 15 anni che viene dalla Siria che ha lasciato il suo paese per fuggire dalla guerra che è ancora in corso. Vive a Milano da circa 2 anni, ma prima di venire in Italia è stata in Giordania. Da quando è qui frequenta la scuola media "Ciresola" e non è mai stata bocciata, ha ancora delle difficoltà in lingua italiana perché confonde qualche parola con l'arabo, ma si impegna molto per superare le sue difficoltà, per esempio facendo molte domande per comprendere il senso di alcuni discorsi e appuntandosi su dei fogli alcune parole che le traduco dall'italiano all'arabo. Desidera molto tornare in Siria ma solo una volta che si sarà sistemata la situazione, infatti più volte mi ha detto che l'unica cosa che le piace di Milano sono i cittadini e i suoi compagni di scuola, ma non le piace la città.

S.: è una ragazza un ragazza marocchina proveniente dalla città di El Jadida ed è qua in Italia da circa 3 anni. Ha 15 anni e ha frequentato per la se- 
conda volta la terza media perché l'anno scorso non è stata ammessa all'esame. Frequenta la scuola "Ciresola" da quando è arrivata e vive qua a Milano con sua mamma. La ragione per cui è emigrata è stata perché sua mamma ha preferito darle il tipo di istruzione che si trova in Italia piuttosto che quella del Marocco. Anche lei, come D., vuole tornare in Marocco perché lì ha lasciato amici e parenti e non le piace vivere qua a Milano. Tuttavia si è ambientata bene, si impegna molto per comunicare correttamente in italiano e ha stretto amicizie soprattutto con ragazzi di origine araba.

E. è un ragazzo egiziano di 15 anni che vive a Milano da quando aveva 10 anni, ha frequentato l'ultimo anno di scuola elementare prima di iscriversi alla scuola media "Ciresola". È venuto in Italia insieme alla sua famiglia per ricongiungersi con alcuni suoi parenti e per avere un'istruzione italiana. Gli piace vivere qua a Milano, ha stretto amicizie con i suoi compagni di scuola di ogni origine, ha poche difficoltà nel capire e nell'esprimersi oralmente in italiano ma ha maggiori difficoltà nello scritto. I primi due anni in cui ha vissuto in Italia ha avuto dei problemi nel relazionarsi con gli altri suoi compagni di classe perché si sentiva in qualche modo discriminato e deriso per le sue origini, ma col tempo ha imparato anche stringere amicizie e a fidarsi degli altri.

A. è una ragazza di origine nigeriana, è qui in Italia da circa 3 anni e vive a Milano con la sua famiglia e frequenta la scuola superiore "Maxwell". Ha un carattere un po' timido perché teme di commettere errori quando parla per cui si concentra molto nello studio. Ha scarsa fiducia nelle sue capacità, forse perché tende sempre a valutarsi tramite i bassi voti scolastici, ma ha delle ottime capacità didattiche che scopre durante i nostri incontri. Ha frequentato il laboratorio parzialmente perché ha anche altri laboratori scolastici da seguire, ma quando può viene volentieri. Con gli altri ragazzi del laboratorio non ha socializzato molto, anche a causa della parziale frequentazione, ha stretto rapporti di più con i tutor. Vorrebbe tornare in visita in Nigeria ma tra un paio di anni per concentrarsi di più sugli studi.

$E$. è un ragazzo ecuadoriano che vive a Milano insieme al fratello e alla madre da circa un anno e frequenta la scuola "Maxwell", in classe con A. Nonostante viva in Italia da poco tempo, parla molto bene l'italiano anche se a volte comunica aiutandosi con delle parole spagnole quando non se le ricorda in italiano. Si è integrato bene con gli altri ragazzi che frequentano il laboratorio perché alcuni già li conosce a scuola, ma in generale è un ragazzo che socializza facilmente con tutti. Ha iniziato a frequentare il laboratorio a circa metà dell'anno scolastico perché non gli interessava e la prima volta è venuto perché spinto dalla sua professoressa di italiano, ma subito dal primo incontro cambiò idea perché scoprì che non era solo un laboratorio di studio pomeridiano come gli era stato riferito.

S. è una ragazza indiana di 17 anni che vive a Milano con la madre e i fratelli da circa un anno e frequenta l'istituto professionale "Bertarelli". La madre è venuta qua in Italia per dare un'istruzione migliore ai propri figli, infatti S. è molto seguita dalla madre nei suoi studi al punto da organizzare delle verifiche con tanto di voto anche a casa. Nonostante parli poco in italiano e vi- 
sto che parlando in inglese molti la capiscono, si è integrata bene con gli altri ragazzi che frequentano il laboratorio. Durante il laboratorio cercava sempre l'attenzione dei tutor per poter studiare e finire i compiti, anche quando il tutor era impegnato con altri ragazzi da seguire. Ha un carattere molto affettuoso e racconta spesso dell'India con nostalgia, e mi ha confessato che Milano non le piace perché è "scarsa di colori" per strada, non come la sua India, dove un giorno spera di ritornare.

\section{Dopo l'esperienza}

\section{Alla fine dell'esperienza, quale valutazione posso esprimere? Sono soddisfatto dell'esperienza che ho compiuto? Perché? Quali sono sta- te le criticità?}

II progetto "Almeno una stella" è stata un'esperienza unica nel suo genere e consiglierei vivamente i ragazzi della mia età di farla. Mi ha aiutata molto a livello professionale in quanto è stata una delle mie prime esperienze lavorative; è grazie a questa esperienza che sto capendo di voler lavorare i futuro con bambini o ragazzi di origine straniera per poterli aiutare a vivere in maniera più serena in un ambiente diverso dalla loro casa.

Essendo anch'io di origine straniera, ho provato empatia con alcuni di questi ragazzi con i loro dubbi sul futuro che avevo anch'io quando avevo la loro età. Trovandosi davanti un tutor che ha superato le loro attuali perplessità, i ragazzi si sentono più sereni e sicuri di potercela fare anche loro per superarle con successo.

Credo che il laboratorio non abbia aiutato solo questi ragazzi, ma anche i tutor a capire come comportarsi con loro e come essere un "modello di riferimento" sia una grande ma piacevole responsabilità. Sentire i ragazzi ringraziarmi per l'aiuto dato mi ha ogni volta sorpresa perché io li aiutavo perché mi faceva piacere oltre al fatto che vedevo che necessitavano del mio sostegno, ma evidentemente per questi ragazzi significava qualcosa di più.

Le criticità ci sono state quando sono arrivati dei ragazzi neo-arrivati che non parlavano proprio italiano o quando alcuni ragazzi hanno abbandonato subito il laboratorio, ma queste difficoltà sono nate solo a causa di una scorretta comunicazione interna della scuola.

\section{Che cosa può fare un tutor secondo me?}

Un tutor deve sostenere i ragazzi nelle loro scelte oltre che nei loro studi. II ruolo del tutor infatti è far capire ai ragazzi che qualunque difficoltà che incontrano è solo momentanea e può essere superata con impegno e dedizione. È importante infondere fiducia nei ragazzi, soprattutto ai neo-arrivati, e nelle loro capacità didattiche dato che trascorrono gran parte del loro tempo tra i banchi di scuola. II tutor deve far capire a questi ragazzi che non hanno minori capacità di apprendimento rispetto ai loro compagni di classe italiani, ma che è normale commettere errori per poter imparare meglio. 


\section{Le proposte che vorrei fare per sostenere l'integrazione delle ragazze e dei ragazzi stranieri}

Credo che il miglior modo per rendere possibile l'integrazione dei ragazzi stranieri siano le attività di gruppo, anche all'aria aperta, insieme ai ragazzi italiani della stessa età. In questo modo si abbassano le differenze culturali esistenti per concentrarsi sull'attività di gioco o studio di gruppo, inoltre così i ragazzi stranieri possono imparare meglio la lingua sentendo come parlano i loro compagni italiani, oltre al fatto che in questo modo anche i neo-arrivati possono applicare la lingua ed essere corretti dai loro coetanei.

Attività come il progetto "Almeno una stella" credo che giochino un ruolo fondamentale nell'integrazione perché è un'attività di gruppo che coinvolge proprio i neo-arrivati, perché è importante che si integrino fin da subito, perché più passa il tempo e più diventa difficile l'integrazione.

\section{Note e considerazioni personali}

Sono grata per aver partecipato a questa attività: ogni studente, tutor, referente scolastico mi ha dato davvero tanto e mi dispiace che sia finito questo progetto. Spero che questo sia solo l'inizio verso una maggiore considerazione istituzionale e professionale del ruolo del tutor nelle scuole e faccia sì che progetti come "Almeno una stella" non siano più solo progetti ma delle realtà della scuola italiana. 


\title{
6. Un compagno, una "spalla", un martello. I modi diversi di essere tutor
}

\author{
di Graziella Favaro, Alessia Marchiò, Horst Wiedemann, \\ Leila Ziglio, Francesca Terenzi e Flavia Virgilio
}

\section{Un prisma a più facce di Graziella Favaro}

Come abbiamo visto, ogni città coinvolta nel progetto ha articolato l'intervento di tutoraggio sulla base delle richieste delle scuole, delle caratteristiche e dei bisogni dei ragazzi accompagnati e delle specificità locali. Questa varietà di situazioni e punti di partenza ha consentito di sperimentare i diversi ruoli del tutor, mettendo l'accento ora sull'una o sull'altra funzione di accompagnamento. Il mosaico delle diversità che emergono serve dunque a comporre il prisma a più facce che descrive il dispositivo del tutoraggio.

Vediamo alcune di queste specificità locali riguardanti, in particolare, gli spazi del tutoraggio, i compiti e gli obiettivi centrali delle azioni, i modi di essere tutor.

\section{I luoghi del tutoraggio}

Nella maggior parte dei casi, il tutoraggio si è svolto negli spazi della scuola, in tempo scolastico o extrascolastico. Questo ha suggerito agli adolescenti accompagnati che il dispositivo era soprattutto scolastico ed aveva a che fare con i compiti, lo studio, gli esami. E ha connotato la relazione iniziale con i tutor, considerati dei "quasi insegnanti" in maniera asimmetrica e distante. Con il tempo, la relazione si è approfondita e "scaldata" e ciò è avvenuto soprattutto negli interstizi del tempo (prima e dopo il laboratorio) e degli spazi (nella strada dalla scuola alla metropolitana). In alcune città invece, il tutoraggio si è realizzato entro spazi più informali e percepiti come propri: nel centro interculturale a Torino, nei locali della "casa delle culture" ad Arezzo, in biblioteca. Qui la relazione è sembrata da subito meno obbligata, più fluida e non regolata e soggetta ai controlli della scuola. I momenti poi di uscita nella città per scoprire i luoghi pub- 
blici e gli spazi di aggregazione per tutti sono stati eventi importanti di vicinanza e hanno provocato spesso un vero e proprio sblocco relazionale.

\section{II patto del tutoraggio}

Tutti i tutor avevano bene in mente che un obiettivo centrale del loro intervento riguardava il sostegno al percorso scolastico in vari momenti: passaggio di scuola, esame di terza media, aiuto allo studio. L'obiettivo poteva tuttavia essere raggiunto attraverso strade e modalità diverse. In alcuni casi, una vicinanza paziente e amicale, volta alla ri-motivazione, ha impedito l'abbandono della scuola da parte di ragazzi a rischio; in altri, un lavoro intelligente di sostegno all'autostima e di valorizzazione dei talenti di ciascuno ha sbloccato situazioni che sembravano di stallo e di sospensione. In altri casi ancora, l'aiuto concreto e quasi quotidiano ai compiti e alle necessità dello studio (gestito anche a distanza) ha consentito di superare difficoltà e muri e di ottenere riconoscimenti dagli insegnanti che hanno prodotto rassicurazione e voglia di continuare.

Il patto di tutoraggio ha messo insieme tutti questi ingredienti e li ha dosati in maniera diversa sulla base dei bisogni degli adolescenti seguiti, delle loro richieste e della loro storia.

\section{Lo stile del tutor}

Ogni tutor ha elaborato un proprio stile di tutoraggio, un modo proprio di stare accanto, di prendersi cura ora in maniera più amicale, ora in modo più distante. Ogni tutor può essere un po' complice e un po' incalzante. Come si legge nella relazione di Arezzo, i tutor possono essere di volta in volta descritti come canguri protettivi, oppure koala dal forte senso materno, come martelli che sollecitano e non perdonano, oppure ancore alle quali aggrapparsi.

Il tema della "giusta distanza" è tornato spesso nelle discussione fra $\mathrm{i}$ tutor e nella fase di formazione e di supervisione. Come essere prossimi e vicini e, nello stesso tempo, autorevoli e degni della fiducia da parte dei ragazzi? Il punto di mediazione e di equilibrio sta forse nelle diverse modalità di stare accanto a seconda dei luoghi e delle situazioni. Nei momenti più formali d'aula e di laboratorio, il tutor scolastico assume un ruolo semiprofessionale, di maggiore distanza e competenze e insiste sul compito comune da affrontare. Nei momenti più informali - prima e dopo l'inizio dei laboratori; nei momenti delle uscite, negli interstizi del tempo insieme - il tutor esprime una vicinanza maggiore e diventa l'amico, la spalla, in confidente. Questo anche grazie alla lingua e ai linguaggi comuni che accorciano le distanze e sciolgono i silenzi.

Nelle pagine che seguono, i racconti degli operatori delle città coinvolte tratteggiano i volti e gli interventi dei tutor dando conto delle diversità e della ricchezza del dispositivo. 


\section{Tutoring e cittadinanza: scoprire la città insieme di Alessia Marchiò}

I torinesi in coda difficilmente aprono bocca. In quanto torinesi, ritengono non lo si debba fare, visto che sia le persone davanti a loro sia le persone dietro a loro appartengono alla categoria "estranei". E se inopinatamente qualcuno davanti o dietro a loro apre bocca, $i$ torinesi lo guardano con l'aria di chi pensa: "Ma cos'ha questo da aprire bocca, in coda, rivolgendo la parola a degli estranei?".

Giuseppe Culicchia, Torino è casa mia

Poi i lampioni della strada si sono illuminati d'improvviso e hanno fatto impallidire le prime stelle che sorgevano nella notte. Ho sentito $i$ miei occhi affaticarsi a guardare $i$ marciapiedi con il loro carico di uomini e di luci. I lampioni facevano luccicare il lastricato umido, e i tram, a intervalli regolari, illuminavano dei capelli lucidi, un sorriso o un braccialetto d'argento. Poco dopo, i tram divenuti più rari e la notte già nera sopra $i$ lampioni e le piante, il sobborgo si è svuotato a poco a poco, fino a che il primo gatto traversò la strada ritornata deserta.

Albert Camus, Lo straniero

Sentirsi estranei o stranieri nella città in cui si vive da pochi mesi, da alcuni anni o nella quale si è addirittura nati è un'esperienza amara, sgradevole, dura da buttar giù, a cui forse ci si abitua, fino a diventare indifferenti, soprattutto se si è giovani adolescenti. In un'età in cui tutti si sentono un po' estranei a se stessi, un po' "strani" e un po' diversi, in una fase della vita in cui la propria personalità è in costruzione, dove si mettono in discussione i principi dell'educazione ricevuta dagli adulti, dove si struttura il proprio sé sociale, il sentirsi stranieri, nel luogo in cui si vive, porta ad acutizzare la sensazione di smarrimento e fragilità che già si prova.

Il progetto "Almeno una stella" nei due anni del suo operato ha cercato, attraverso l'azione quotidiana dei tutor, di smuovere e di contrastare questa condizione, creando nuove consapevolezze e diversi vissuti, scoprendo la città insieme.

* Centro Interculturale della Città di Torino e Associazione ASAI. 
La relazione tutor-tutorato, che si è consolidata nei due anni di progetto, si è basata sull'intimità della conoscenza reciproca, in un rapporto non simmetrico, impari e sbilanciato, per alcuni aspetti (ad esempio, quelli delle competenze e della differente maturità psicologica) ma equamente significativo e denso di affettività. La relazione che si è costruita e rinforzata pazientemente, giorno dopo giorno, insieme, nei momenti deputati ai compiti, allo studio, al corso di italiano, ha favorito nei giovani studenti l'acquisizione e lo sviluppo delle proprie competenze. Queste capacità sono legate al sapere e hanno condotto, nella maggior parte dei casi, ad un miglioramento dei risultati scolastici; esse sono state veicolate, a volte anche inconsapevolmente, da altri fattori: si sono potute concretizzare perché tutt'intorno si è costituita una cornice, una struttura solida, portante, fatta di dialogo, di confidenze, di richieste di aiuto da un lato, e di ascolto, cura, protezione dall'altra parte.

Le competenze scolastiche e i positivi risultati ottenuti a scuola dai ragazzi sono perciò stati affiancati dal parallelo sviluppo di altre abilità ugualmente importanti: le competenze relazionali e sociali. Attraverso l'esempio e la condivisione di esperienze, i tutor hanno portato i ragazzi a scoprire dentro di sé capacità e qualità ignorate e spesso sottostimate, aiutandoli a cogliere molteplici opportunità, grazie a una nuova fiducia in se stessi.

L'obiettivo di scoprire la città insieme rientra nel quadro descritto di ampliamento delle risorse, delle capacità dei ragazzi, della loro autonomia, mediante la consapevolezza di essere cittadini di una città, che può essere conosciuta e che può diventare in qualche modo più "nostra".

Il ruolo del tutor è stato quello di accompagnare nella conoscenza del territorio, di condividere esperienze e vissuti significativi, di promuovere lo scambio interculturale e l'integrazione nel contesto urbano.

Fin dalla prima annualità di progetto, i tutor hanno indagato il rapporto dei ragazzi di "Almeno una stella" con il territorio cittadino attraverso un questionario (vedi all. 8 in fondo al testo). In esso, si chiedeva ai giovani tutorati quali luoghi della città conoscessero, dove andassero nel tempo libero, se si muovessero autonomamente con i mezzi pubblici, se avessero visitato monumenti importanti dal profilo storico-artistico, ecc. Le risposte che erano emerse dall'analisi dei questionari indicavano come molti ragazzi vivessero confinati nel quartiere in cui risiedono e dove vanno a scuola, e che della città di Torino conoscessero e frequentassero principalmente le vie dello shopping, i centri commerciali e alcuni parchi pubblici vicino a casa.

Insieme ai tutor sono perciò state pensate delle proposte per espandere i confini del territorio in cui si muovono i ragazzi, per ampliare il raggio di azione dei loro spostamenti e, soprattutto, per diversificare la tipologia di 
uscite, svincolandole da modelli di consumismo immediato, dalla frequentazione di non-luoghi che non apportano valore aggiunto o possibilità di crescita, scambio e confronto.

\section{Torino è casa mia}

Nei due anni di "Almeno una stella", i tutor hanno accompagnato i ragazzi alla scoperta di nuovi posti della città, al di fuori dei limiti dei quartieri periferici in cui vivono o dei luoghi abitualmente deputati al divertimento. Non si è trattato di far vivere ai giovani studenti esperienze eccezionali, fuori dal quotidiano, ma di trovare insieme dei servizi, degli spazi e delle offerte culturali e artistiche che la città mette a disposizione, spesso gratuitamente, e che basta solo "imparare" a cogliere. Lo scopo delle proposte effettuate si inscrive nell'ottica di favorire l'autonomia, di condurre i ragazzi a delle attività che potranno ripetere in futuro, ripercorrendo da soli il sentiero su cui i tutor li hanno portati per mano, nella convinzione che le attività svolte insieme possano incidere e radicarsi nel vissuto individuale dei ragazzi, consolidando pratiche positive.

Gli studenti hanno partecipato alla rassegna cinematografica gratuita Mondi lontani mondi vicini presso il Cinema Massimo, hanno visitato il Museo Nazionale del Cinema presso la Mole Antonelliana, hanno conosciuto la Bibliomediateca del Museo del cinema, hanno fatto gite in piscina $\mathrm{o}$ in bicicletta nei parchi cittadini.

Hanno riscosso particolare successo la visione dei film della Rassegna, ricchi di tematiche interculturali in cui i ragazzi si sono identificati, e la promozione della Bibliomediateca Mario Gromo, realizzata mediante la presentazione del libro Fuori piove, dentro pure, passo a prenderti? scritto da un giovane autore migrante, Dikele Distefano.

I tutor hanno favorito la costruzione di un dialogo interculturale, condividendo valori e confrontando esperienze: nella pratica quotidiana del Circle Time, con cui iniziavano gli incontri di sostegno allo studio, tutti in cerchio a condividere piccoli fatti o riflessioni personali con il gruppo; con i momenti di aggregazione, dibattito e confronto preparatori o a seguito delle iniziative loro proposte. Particolarmente importanti sono state le passeggiate alla scoperta della città, dove i ragazzi hanno esperito (sotto forma di giochi, attività di orienteering e caccia al tesoro) dei percorsi di conoscenza di Torino, della sua storia, delle risorse che essa offre ai giovani, osservando l'architettura urbana, ascoltando la voce dei suoi abitanti, venendo a conoscenza delle associazioni che promuovono una cittadinanza attiva attraverso lo scambio interculturale, la promozione di eventi e attività artistiche e culturali, il diffondersi di una mentalità più aperta allo scambio e al confronto. Prima di proporre tali attività agli studenti, i tutor hanno 
preparato un gioco, ispirandosi al libro di Giuseppe Culicchia Torino è casa mia e a quello di Francesco Vietti Torino è casa nostra: i ragazzi sono stati divisi in due squadre e hanno realizzato due cartelloni che rappresentano la piantina di un appartamento, dove hanno definito quali luoghi della casa corrispondano per loro a luoghi della città (quali quartieri sono la cucina, la camera da letto, il bagno, il giardino della città, ecc.).

Molto rilevanti dal punto di vista dell'interculturalità sono state le visite svolte nelle due passeggiate, una attraverso il quartiere di Barriera/Regio Parco, l'altra nel quartiere di San Salvario, alla scoperta dei luoghi della spiritualità. I ragazzi, infatti, hanno avuto l'opportunità di visitare una chiesa, una sala di preghiera islamica, la sinagoga e il tempio valdese, partecipando con interesse e con rispetto dei differenti modelli religioso-culturali di cui loro stessi, per primi, sono portatori.

\section{Linguaggi espressivi meticci}

La promozione della cittadinanza da parte dei tutor nei confronti dei tutorati non è stata a senso unico: così come gli studenti hanno scoperto nuove parti di Torino, così la città ha scoperto nuovi giovani cittadini. I tutor hanno accompagnato i ragazzi a diventare protagonisti della città in cui vivono, esprimendo se stessi attraverso linguaggi artistici, mettendo in gioco emozioni e competenze. La partecipazione alla "Giornata internazionale della Lingua Madre", tenutasi il 20 febbraio 2015 presso la Sala del Consiglio Comunale, ha avuto la finalità, dinanzi al pubblico della città, attraverso esibizioni musicali, letture in prosa e poesia nella propria lingua madre, di implementare nei ragazzi la fiducia in se stessi, valorizzando la cultura di appartenenza e le proprie abilità.

A partire da marzo 2015, inoltre, i ragazzi hanno cooperato alla realizzazione di un prodotto artistico che li vede protagonisti di un evento in un teatro cittadino, in occasione di Feed the Cities, progetto promosso dall'Assessorato alla Cultura, in collegamento con la chiusura di Expo Milano 2015. La scelta di proporre ai ragazzi il teatro è nata per perseguire obiettivi educativi e culturali, offrendo loro uno spazio di espressione di sé, protagonismo e una possibilità di crescita personale: si tratta di un'esperienza stimolante, basata sulla relazione con il gruppo (il teatro è socialità, scelta di stare insieme, conoscenza dell'altro e di sé), che si attua mediante un costante lavoro sulle proprie emozioni. È un percorso che arricchisce gli strumenti linguistici del ragazzo e concorre alla formazione di una personalità armonica, puntando sul divertimento e il coinvolgimento.

Il binomio tutoring-cittadinanza ha coinciso perciò con un cammino progressivo, per riappropriarsi degli spazi della città in cui si vive, per non sentirsi più estranei/strani/stranieri in essa: ha rappresentato un'opportunità 
di conoscenza reciproca tra tutor e tutorati per la costruzione di relazioni significative, una possibilità per imparare a dialogare tra persone portatrici di culture diverse, un percorso per esprimere se stessi e "tirar fuori" la propria personalità.

La relazione che si è instaurata, nelle sue molteplici sfumature individuali e soggettive, tra tutor e studenti, è resa bene dall'etimologia della parola "tutor", derivante dal latino "tutari", che significa "proteggere", "difendere", "custodire". I tutor hanno protetto, difeso e custodito i giovani adolescenti, ne hanno avuto cura, accompagnandoli per un tratto del loro percorso di vita, ricevendo in cambio un'occasione di crescita personale e una grande ricchezza, fatta di affetto e di stima. 


\section{Gli aspetti relazionali del tutoring scolastico di Horst Wiedemann*}

Bologna, a differenza delle altre città che hanno privilegiato studenti universitari, fin dall'inizio aveva optato per la figura del tutor scolastico individuato tra gli studenti del terzo anno delle scuole superiori, prioritariamente tra gli studenti stranieri ma anche con una discreta presenza di tutor italiani.

I tutor hanno rappresentato per i tutorati i compagni di scuola più grandi, quelli che ce l'hanno fatta a diventare "bravi alunni" sul piano delle discipline, ma anche sul piano della maturazione personale e delle competenze sociali, quelli che possono quindi far da modelli con cui identificarsi. Sulla base delle loro esperienze fatte nel medesimo istituto, è facile per loro avvicinare il ragazzo neoarrivato alla nuova realtà. Rispetto a figure adulte possono allo stesso tempo stabilire relazioni personali più libere e intense vista la differenza contenuta di età e l'informalità dei loro contatti.

Con la grinta del loro essere bravi studenti e la forza della propria esperienza, hanno potuto sostenere la motivazione e la voglia di riuscire dei loro tutorati e calarsi più facilmente e con empatia nei vissuti di disorientamento e frustrazione che spesso connotano le situazioni dei nuovi arrivati. Essendo abituati allo specifico contesto scolastico con le sue regole, consuetudini e materie e conoscendo spesso di persona i professori e le loro modalità didattiche e di valutazione, sono stati in grado di aiutare i loro compagni con consigli concreti ed efficaci e di trasmettere loro strategie e stratagemmi di "sopravvivenza a scuola".

Gli abbinamenti tra tutor e tutorati spesso sono stati decisivi per l'efficacia dell'intervento. Hanno giocato fattori come l'indirizzo di studi seguito all'interno dello stesso Istituto, la propensione per certe materie scolastiche e la capacità di padroneggiare certi argomenti disciplinari da parte dei tutor, la possibilità o meno di usare la lingua d'origine o le lingue straniere studiate a scuola come lingua ponte. Ma anche l'essere maschi o femmine, la provenienza nazionale con le particolarità culturali, religiosi e valoriali hanno avuto importanza in relazione alle culture ed esperienze familiari d'origine.

La decisione su chi abbinare con chi si è rivelata piuttosto delicata e spesso decisiva per l'efficacia dell'intervento.

Come criteri immediati abbiamo tenuto conto senz'altro di fattori "tecnici" come l'indirizzo di studi, la propensione per certe materie scolastiche e la capacità di padroneggiare certi argomenti disciplinari da parte dei tutor,

* Coordinamento pedagogico di "Almeno una stella", Bologna. 
la possibilità o meno di usare la lingua d'origine o le lingue straniere come lingue veicolari.

Più di tutto però hanno contato ovviamente le caratteristiche personali di ogni singolo, tutor o tutorato che sia. Tra tutor e tutorati in questi due anni si sono sperimentati incontri "perfetti", sia dal lato relazionale che da quello didattico, rapporti con alti e bassi, e anche alcuni abbinamenti problematici. Ogni rapporto di tutoraggio è quindi una appassionante storia a sé.

Gli aspetti relazionali sono stati altrettanto importanti quanto le questioni didattiche: ascoltare e far parlare i ragazzi liberamente della loro vita, incoraggiarli a superare momenti di stanchezza e rafforzare la fiducia in loro stessi, valorizzare i progressi, trovare i modi per ottenere interesse, attenzione e impegno. La carica emotiva ha dato forza anche all'apprendimento: sorridere, scherzare, introdurre variazioni di tono e di ritmo, modulare i tempi, trasmettere sicurezza e tranquillità, ascoltare l'altro, ma anche raccontare di se stessi: tutto questo ha alleggerito le sistuazioni e sostenuto l'apprendimento.

\section{A scuola e fuori dalla scuola}

Il contesto operativo principale per l'intervento dei tutor sono stati i laboratori di italiano L2 e di sostegno didattico nei vari Istituti, soprattutto nella fase di avvio dell'anno scolastico. I laboratori hanno costituito una cornice ben strutturata, grazie alla presenza di figure esperte, e hanno permesso ai tutor ancora inesperti di operare in affincamento ai docenti.

Con la crescita della loro autonomia operativa e il consolidamento dei rapporti coi tutorati, in seguito i tutor hanno usato sempre di più anche altre modalità per seguire i loro tutorati: luoghi più informali come le biblioteche di quartiere, casa propria e/o del tutorato, oppure certi "interstizi" della vita scolastica come l'ora di religione, gli intervalli, il momento dell'uscita da scuola. Hanno sperimentato diverse forme di accompagnamento, dal rapporto individuale al gruppo allargato, lavorando anche in squadra con gli altri tutor. I tutor hanno sperimentato maggiormente momenti autogestiti organizzandosi nelle singole scuole come gruppo intero o parziale, potendo in questo modo aiutarsi a vicenda e anche "scambiarsi" i loro tutorati in base alle loro capacità, competenze e provenienze linguistiche.

L'ampia gamma di modalità di interazione ha favorito senz'altro la risposta a bisogni che erano molto differenziati e ha permesso di personalizzare maggiormente le azioni. Nelle situazioni di compresenza e di collaborazione tra docenti esperti, tutor e (dove era possibile) docenti disciplinari, in un approccio laboratoriale condiviso, è stata possibile una maggiore 
individualizzazione degli interventi e si è potuto rispondere in maniera più efficace alle esigenze specifiche degli studenti. La pluralità, non solo delle competenze, ma anche delle professionalità è stato un elemento di stimolo relazionale oltre che didattico. In particolare, la presenza dei tutor ha creato un clima più amichevole: su certi temi e difficoltà per i ragazzi è più facile rapportarsi con altri giovani quasi coetanei perché più simili e più vicini sono le sensibilità e le modalità comunicative. L'obiettivo primario dei tutor nei confronti dei compagni di scuola era infatti quello di rinforzare in questi ragazzi il senso di fiducia in se stessi e nella effettiva possibilità di recupero e di miglioramento scolastico. I ragazzi tutor hanno potuto usare le loro conoscenze disciplinari e competenze di metodo di studio e hanno cercato di proporre una comunicazione didattica efficace, ma prima di tutto hanno cercato di impostare un approccio accogliente e motivante sul piano personale e di piccolo gruppo. Ogni tutor aveva inoltre capacità e competenze diverse e ha potuto conseguentemente essere di particolare aiuto in certe discipline.

Purtroppo, solo dopo diversi mesi, sia nella prima che nella seconda annualità del progetto si è raggiunta una discreta "tenuta" degli abbinamenti. La continuità relazionale in molti casi ha prodotto anche il raggiungimento di risultati scolastici positivi. Ragazzi che ce l'hanno fattta dove prima non riuscivano, ragazzi che hanno sviluppato una motivazione quando prima c'era chiusura o rifiuto, ragazzi che hanno acquisito sicurezza e autonomia mentre prima evitavano o fallivano. Molti dei ragazzi seguiti hanno avuto riscontri positivi nelle verifiche e nelle interrogazioni in alcune materie. Ottenere dei risultati concreti di cui essere soddisfatti dà poi ai ragazzi la misura dell'utilità dell'intervento e accresciuto la motivazione dell'intero gruppo, sia dei tutor che dei tutorati.

\section{Percorsi di formazione condivisa}

Una serie di incontri formativi rivolti ai tutor delle scuole bolognesi ha consentito il formarsi di un gruppo tutororaggio cittadino. Tra i ragazzi si è creato un buon affiatamento e la percezione di essere un'unica squadra con la voglia di condividere l'esperienza e di imparare gli uni dagli altri. Scopo degli incontri formativi non era tanto l'acquisizione di competenze professionali "da tutor", quanto il confronto e lo scambio tra di loro e la consapevolezza di essere - come cittadini del mondo - parte attiva nella loro scuola e nella loro città. In alcuni degli incontri, sono stati coivolti anche alcuni tutorati, ad esempio quando per la videodocumentazione si è trattato di simulare situazioni tipo di tutoraggio.

L'esperienza si è rivelata anche una formidabile occasione di crescita e di arricchimento per i tutor. 
Ha dato loro l'opportunità di sperimentarsi in un ruolo complesso e di rilevanza sociale che richiede impegno, autonomia, assunzione di responsabilità.

Nel corso del progetto, sono state sperimentate anche modalità di formazione integrata docenti-tutor. Un esempio è l'incontro tra 6 docenti dell'Istituto "Aldrovandi" e 11 tutor (provenienti anche dalle altre 3 scuole superiori coinvolte), finalizzato al confronto tra le modalità di gestione operativa del dispositivo di tutoring. In quell'occasione, i tutor sono stati i protagonisti della "narrazione", in quanto ormai "esperti di materia" e insieme ai docenti hanno evidenziato anche alcuni punti centrali per la qualità delle relazioni tra loro e i ragazzi seguiti.

Eccoli di seguito:

- I tutor hanno diverse preferenze, propensioni e capacità personali e quindi possono accogliere una grande varietà di bisogni e tentare di dare risposte personalizzate.

- Possono ascoltare e far parlare i ragazzi tutorati liberamente della loro vita, incoraggiarli per superare problemi di scarsa motivazione e di perdita di fiducia in se stesso, valorizzare i progressi, trovare i modi per ottenere interesse, attenzione e impegno dai ragazzi.

- II tutoraggio evidenzia l'importanza della carica emotiva per dare energia alla relazione didattica (sorridere, usare un tono euforico, istillare sicurezza e tranquillità, raccontare di se stessi...).

- Il tutoraggio evidenzia l'importanza della facilitazione: usare parole semplici, ripetere, far provare e riprovare.

- II tutor deve cercare di fungere anche come mediatore/stimolatore/catalizzatore dell'aiuto reciproco che possono darsi i ragazzi tutorati e organizzare momenti di lavoro cooperativo.

- Nel tutoraggio vi sono anche aspetti problematici: ci sono casi di poca motivazione nei ragazzi che certamente non sono risolvibili dal solo intervento del tutor; è talvolta difficile trovare i momenti per incontrarsi, soprattutto per i ragazzi che non seguono i corsi di italiano o di sostegno didattico; il sovrapporsi di numerosi impegni sia dei tutor che dei tutorati, spesso rende difficile realizzare la continuità auspicata. Inoltre manca talvolta un'effettiva condivisione e integrazione dei diversi dispositivi di sostegno e potenziamento tra i diversi operatori della scuola: docenti, esperti italiano L2 e mediatori. 


\section{Costruire la resilienza attraverso il tutoring di Leila Ziglio*}

\section{La specificità del contesto trentino}

In provincia di Trento, il progetto "Almeno una stella" è stato realizzato nel corso di due anni scolastici e ha subìto degli adattamenti rispetto al progetto nazionale, dovuti alla specificità del contesto e del territorio. Fin dall'inizio ha coinvolto parallelamente alcuni studenti che dovevano affrontare l'esame di stato al termine del primo ciclo d'istruzione e altri frequentanti il primo biennio delle scuole secondarie di secondo grado e della Formazione professionale. Di questi ultimi alcuni sono stati seguiti anche in seconda superiore.

I Centri di formazione professionale e gli Istituti superiori sono collocati in cittadine (Pergine Valsugana ha 21.000 abitanti, Borgo e Levico Valsugana, circa 7.000 ciascuno), gli Istituti comprensivi anche in piccoli paesi, distribuiti su un territorio abbastanza ampio, quello della Valsugana.

Ogni tutor ha affiancato uno o due studenti e i percorsi di tutoring sono stati fortemente personalizzati, sia perché i bisogni espressi dai singoli soggetti erano molto diversi tra loro, sia perché non è stato possibile ancorare il tutoraggio a realtà di laboratorio d'italiano L2. Tutti gli incontri si sono svolti in orario extrascolastico e, per le scuole superiori, solo saltuariamente nelle sedi degli Istituti.

Uno staff di progetto di quattro coordinatrici si è occupato della supervisione del lavoro dei tutor mediante incontri periodici e, su loro richiesta, è sempre stato disponibile a incontri individuali e a consulenze informali tramite mail o cellulare. Lo stesso staff ha mantenuto anche un rapporto e un confronto costante con le scuole, in particolare con i referenti per le iniziative interculturali e i coordinatori di classe, e ha cercato di incidere, con consulenze e strumenti per la personalizzazione dei percorsi e la conseguente valutazione, sulle modalità d'intervento delle scuole.

Nel 2013-14 solo un terzo dei partecipanti al progetto era neo-arrivato (NAI); nell'anno in corso la loro percentuale era calata al 28\%. La maggior parte dei ragazzi e delle ragazze è quindi di immigrazione meno recente o di seconda generazione.

Le difficoltà maggiori non sono state registrate con i ragazzi e le ragazze neo-arrivati. Nella nostra esperienza, spesso il primo anno di scolarizzazione in Italia presenta minori difficoltà di quelli successivi, perché la scuola, a fronte della complessità che scaturisce da un trasferimento in un Paese straniero in età adolescenziale, utilizza dispositivi ormai consolidati, mentre è meno attrezzata nei confronti dei ragazzi qui da più tempo.

* Centro Interculturale Millevoci - Provincia di Trento. 
Queste ragioni ci hanno spinto a mettere in primo piano il tema della resilienza, inteso come lavoro sulla capacità di affrontare e superare ostacoli e di riprendere un cammino interrotto. Inoltre, la resilienza è sottolineata, come aspetto importante, nel report del progetto di ricerca precedente a questo (Favaro, 2013), e più autori riconoscono la migrazione come uno tra i fattori di rischio che espongono gli adolescenti alla probabilità di sviluppare problemi di adattamento sociale.

\section{II lavoro sulla resilienza}

La condivisione della definizione di "tutore di resilienza" (Cyrulnik 2005) è stata al centro di un modulo di formazione che ha coinvolto attivamente i tutor e che è stato seguito da un altro dedicato ai giovani adolescenti di origine migrante e alle loro famiglie. Nel primo anno di progetto lo staff ha predisposto una scheda, su cui i tutor e le tutor hanno lavorato individualmente e in piccoli gruppi, riferendo a se stessi e alla propria storia di vita l'analisi delle doti di resilienza. Questa riflessione concreta, seguita dall'esposizione teorica, ha poi consentito loro di riconoscere nei ragazzi tutorati le attitudini alla resilienza e di promuoverne il rafforzamento, usando anche se stessi e la propria esperienza come fulcro su cui fare leva per motivarli e incoraggiarli.

Nel secondo anno di ricerca è stata preparata, con la supervisione di Graziella Favaro, anche una scheda per l'osservazione delle caratteristiche di resilienza dei ragazzi coinvolti nel progetto. Questo nuovo strumento di riflessione sul tema (vedi all. 7 in fondo al testo) ha indirizzato il lavoro dei tutor e ha permesso di raccogliere le loro considerazioni in merito alla resilienza degli studenti che avevano in carico. La scheda, pensata e scritta come una traccia d'intervista, è stata oggetto di riflessione comune tra tutor e formatrici. Sono state date ai tutor alcune indicazioni: di non utilizzare la scheda come un questionario; di iniziare da eventuali osservazioni sulla resilienza già scritte nel diario di bordo, strumento di documentazione degli interventi che riporta anche la descrizione degli incontri e il racconto della storia dei ragazzi seguiti; di evitare forzature, nell'usuale rispetto dei limiti posti da ragazzi e ragazze alla narrazione di se stessi e del proprio vissuto. È stata data loro totale autonomia in merito alla raccolta dei dati, ritenendoli in grado di valutare le modalità più adatte, in base alle diverse circostanze, al rapporto esistente e al grado di confidenza raggiunto con i giovani tutorati. E così è accaduto nei fatti. Alcuni tutor erano già in possesso di tutte le informazioni richieste e hanno ricostruito il quadro delle doti di resilienza basandosi sulla conoscenza diretta, sui racconti di vita e sulle informazioni che avevano annotato. Altri hanno rivolto ai ragazzi delle domande esplicite, altri ancora hanno deciso che non esistevano le 
condizioni per parlarne apertamente e hanno riferito quanto già sapevano o i comportamenti osservati. Alla domanda sulle intelligenze multiple, in quasi tutti i casi, è stata data risposta dai tutor.

Brani tratti dai diari di bordo dei tutor integrano il presente testo per illustrare con immediatezza ed efficacia quanto scritto.

\section{II concetto di resilienza}

Non è forte chi non cade mai, ma chi, cadendo, si rialza.

J.W. von Goethe

Il significato originale della parola resilienza viene dalla fisica dei materiali e indica la capacità di un corpo di assorbire colpi e deformazioni da parte di forze esterne senza rompersi e di riacquistare, in seguito, la propria forma.

Il termine è divenuto una metafora ed è stato assunto dalla psicologia per indicare l'attitudine di una persona a superare, positivamente e in una prospettiva di crescita personale, le esperienze traumatiche che la vita può presentare.

Di seguito, si cerca di sintetizzare un dibattito complesso, facendo riferimento principalmente all'impianto teorico proposto nei testi di B. Cyrulnik (2003 e 2005) e di E. Malaguti (2005).

Gli studi sulla resilienza hanno avuto origine da una ricerca trentennale sulla vulnerabilità, condotta da una psicologa statunitense, Emmy Werner (Werner e Smith 1982), che ha indagato i fattori e le condizioni che facilitano, nei bambini, lo sviluppo delle capacità di reazione e delle qualità atte a trasformare un'esperienza dolorosa in apprendimento per la vita.

\section{La vulnerabilità}

La vulnerabilità è definita da Werner e Smith come la predisposizione a essere facilmente feriti e a sviluppare disturbi di comportamento e di adattamento, in presenza di fattori di rischio e di eventi negativi. Studi successivi (Anaut, 2003) hanno posto in evidenza che i fattori di rischio che predispongono alla vulnerabilità non possono essere riferiti solo alle caratteristiche del singolo individuo, ma sono connessi anche alle debolezze e alle deprivazioni dell'ambiente familiare e sociale in cui vive.

Nel caso specifico degli adolescenti neoarrivati, la vulnerabilità va riferita alla loro particolare situazione e va intesa come condizione temporanea, legata alla migrazione, come squilibrio transitorio tra i compiti da affrontare e le risorse per farvi fronte. Queste possono essere sia qualità personali, sia opportunità messe a disposizione dal contesto sociale, inteso 
come famiglia, gruppo dei pari, ambito scolastico, ambito sociale e territoriale, ecc. Gli elementi di rischio sono connessi al loro migrare "forzato", non scelto, da adolescenti e all'involuzione e alla regressione che subiscono la loro possibilità di esprimersi, il loro rapporto con il sociale e, non ultimo, il loro profilo scolastico.

E., arrivato a 17 anni per frequentare la scuola alberghiera, mi ha raccontato del grande accumulo di sconforto, senso di abbandono, solitudine vissuti al momento del suo arrivo in Italia. Il passaggio da una realtà in cui era perfettamente integrato nella rete di amici, con una famiglia presente e rassicurante, a un mondo totalmente nuovo, privo di legami, dove avrebbe dovuto ricominciare da capo, dove si parla una lingua oscura, che l'ha portato a chiudersi in se stesso, in uno stato di scoraggiamento e noia. Questo è durato per alcuni mesi, poi, nel momento in cui ha conosciuto un ragazzo albanese come lui, già da molto tempo in Italia, ha iniziato, col suo aiuto, ad addentrarsi nella nuova realtà, creando pian piano legami. L'amico ha fatto da "ponte" per il cammino di E. (Dal diario di bordo di A.)

La vulnerabilità in età adolescenziale dei giovani di remota immigrazione e di seconda generazione presenta caratteristiche in parte diverse, perché perde il carattere di temporaneità, persiste nel tempo e tende a radicarsi nei comportamenti. In questo senso è più complessa da affrontare $\mathrm{e}$ richiede un percorso spesso più lungo e più incerto rispetto alle possibilità di riuscita.

Chi appartiene alle seconde generazioni, a differenza dei genitori, percepisce il Paese in cui vive, in questo caso l'Italia, come il proprio paese, "si sente italiano", e questo determina modalità d'integrazione e comportamenti autonomi e dissimili da quelli della generazione precedente. Questi ragazzi vivono una dissonanza sia con le figure parentali, sia con molti italiani, che non riconoscono loro lo status di cittadini e li etichettano come stranieri.

Entrambi i ragazzi che ho seguito sono nati in Italia da genitori marocchini. Vivono un conflitto costante tra la vita del Paese in cui sono nati e dove si stanno formando, assumendone usi, costumi e leggi, e la cultura e tradizione portata avanti dalle famiglie d'origine. (Dal diario di bordo di J.)

Le cose cambiano quando incontriamo i ragazzi di seconda generazione, i figli dei migranti: sono nati e cresciuti qui, qui vanno a scuola, creano relazioni e legami. Non sono migranti, perché non si sono mai spostati. Eppure restano stranieri, e spesso sono stranieri anche nel paese d'origine: ne conoscono poco le usanze, le tradizioni, a volte anche la lingua. (Dal diario di bordo di A.)

Questa discrepanza è spesso denunciata nei loro discorsi: "Quando sono in Marocco mi chiamano italiano, qui in Italia mi dicono marocchino". (Dal diario di bordo di C.) 
A questo si aggiunga che quasi tutte le famiglie migranti vivono dei momenti di crisi e di conflitto, che nascono dalla necessità di ridefinire funzioni, ruoli e competenze dei vari membri della famiglia e di impostare nuovi stili di vita in emigrazione. Se la struttura familiare e le regole di relazione che la definiscono (Jackson, 1965) non sono abbastanza flessibili da rendere possibili delle trasformazioni, rischiano d'impedire i processi di cambiamento, mentre è importante che la famiglia immigrata trovi, nel tempo, un equilibrio tra continuità col passato e integrazione nella realtà presente. Figli e figlie nella migrazione spesso vivono in un ambiente familiare conflittuale, non di rado incapace di aiutarli, che genera in loro sofferenza; inoltre, in parecchi casi, si trovano a fare i conti con nuove figure parentali.

Dopo l'abbandono della famiglia da parte del padre, M. arriva in Italia a 15 anni con la madre, che si risposa in breve tempo con un uomo italiano, con cui la ragazza non va d'accordo. M., che in Romania era tra le più brave della classe, ha difficoltà a scuola e viene respinta; questo la rende sfiduciata e insicura. Le viene diagnosticata una lieve forma di depressione. Mi confida che le difficoltà della sua famiglia influiscono molto sul suo impegno scolastico, che diventa ancora più scarso nel periodo, travagliato, in cui la madre si separa dal nuovo marito. Anche il suo rapporto con la madre s'inasprisce. (Dal diario di bordo di V.)

\section{I fattori di protezione}

L'esistenza di fattori di protezione non può essere intesa in senso determinista e predittivo, poiché le variabili che influiscono sullo sviluppo di doti di resilienza sono molteplici e complesse. Lo stesso vale per i fattori di rischio e la possibilità che producano disadattamento. Inoltre, sia i fattori che predispongono alla vulnerabilità, sia i meccanismi di protezione non operano singolarmente, ma interagiscono tra loro in modo dinamico.

Questa premessa (Garmezy, 1991) aiuta a definire i fattori protettivi come quegli elementi, di carattere individuale, familiare o sociale, che possono promuovere negli individui lo sviluppo della resilienza.

I fattori protettivi individuali riguardano la personalità dell'individuo e la capacità di riflettere su se stessi. Quelli menzionati da più autori sono:

- la fiducia in sé e nelle proprie risorse;

- l'ottimismo e la capacità di considerare le avversità come fattori temporanei e superabili;

- l'accettazione del cambiamento come parte della vita;

- la determinazione rispetto ai propri obiettivi e la capacità di perseguirli concretamente, passo dopo passo;

- la capacità di comunicare e di socializzare;

- la capacità di ridere di se stessi e delle proprie difficoltà. 
Trovare il senso dell'umorismo tra le qualità necessarie a sviluppare resilienza può forse destare stupore. Lo humor permette di non prendersi troppo sul serio e toglie coinvolgimento rispetto a ciò che ci accade; è la capacità di guardare noi stessi e le nostre vicende, anche tragiche, da una certa distanza, cogliendone gli aspetti comici e grotteschi. È uno dei modi per conservare la dignità nei momenti difficili.

Le competenze e gli atteggiamenti riportati sopra, che alcuni soggetti maturano senza troppo sforzo, possono essere costruiti e sviluppati da ogni individuo. Gli studiosi e i ricercatori pongono fortemente l'accento sul fatto che la resilienza consiste principalmente in un insieme di pensieri e comportamenti che possono essere appresi e rafforzati nell'ambito di processi educativi, cioè intersoggettivi e finalizzati a promuovere la crescita di un individuo. E il rapporto tra tutor e adolescenti di origine straniera è di carattere educativo.

I fattori protettivi esterni sono le condizioni che dipendono dall'interazione con l'ambiente, familiare o extra-familiare, rilevate e identificate per la prima volta da $\mathrm{E}$. Werner. Tra questi, quelli riscontrati da più ricerche $\mathrm{e}$ ritenuti essenziali sono una relazione affettiva stabile, il confronto con una o più figure di riferimento e poter contare su reti sociali di appoggio. B. Cyrulnik ribadisce costantemente che la creazione di legami significativi è una condizione indispensabile per divenire resilienti. Dopo che l'importanza di questi fattori fu riconosciuta, gli psicologi iniziarono a lavorare sulla loro trasformazione da elementi casuali in intenzionali, cioè in strumenti che possono essere progettati e messi in atto da persone o da ambienti interessati a contribuire al rafforzamento delle capacità psicologiche di un individuo.

In sintesi, la resilienza si configura come un agire pratico, un dato esperienziale che richiede riflessione, intuizione, conoscenza e ascolto di se stessi, e uno o più sostegni esterni.

Il processo di adattamento di fronte alle avversità si sviluppa in tempi e modi diversi per ognuno e prevede alcune tappe fondamentali:

- resistere e non farsi travolgere da traumi ed altri eventi critici;

- integrare il dolore, viverlo, e imparare a conviverci, perché in parte può permanere;

- saper guardare oltre e considerare gli accadimenti in una prospettiva diversa;

- risollevarsi e riuscire a ri-elaborare e a ri-organizzare il proprio percorso di vita, trasformando l'evento traumatico in un'occasione di cambiamento.

La resistenza è una fase necessaria per opporsi alle circostanze avverse, un atteggiamento di difesa che comporta anche rigidità e non aiuta l'accettazione di quanto sta accadendo; allo stesso modo il dolore, se è negato e 
non accolto, non viene metabolizzato e trasformato. La resilienza richiede flessibilità ed elasticità, qualità dinamiche che, sommate ad altre capacità, consentono alle persone di contrastare gli eventi e, in seguito, di andare avanti senza arrendersi e senza irrigidirsi.

B. Cyrulnik (2005) afferma che nessuno diventa resiliente da solo e sottolinea il fatto che la resilienza non si ottiene facendo leva solo sulle doti individuali, ma dipende dalla possibilità di riallacciare i legami con una comunità che si percepisce come propria e in cui si può ricominciare a ricostruire.

La funzione del tutor, importante in ogni fase del processo, diventa fondamentale nello stadio della ristrutturazione del proprio percorso esistenziale, sia nel sostenere l'individuo come singolo, sia nel favorirne l'interazione con il contesto sociale.

\section{La scuola come un possibile fattore di protezione}

Le istituzioni scolastiche, a seconda della loro capacità d'integrazione e di tutela di soggetti deboli, quali i ragazzi migranti, possono contribuire o meno ad alleviarne le condizioni di vulnerabilità e a renderle meno pesanti. Una delle ragioni del progetto sta nella convinzione che la scuola sia una delle agenzie che possono sostenere persone vulnerabili in un processo di resilienza e che vada rafforzata in questo compito, che non sempre riesce a portare a termine da sola. A questo proposito una tutor italo-macedone ci ha raccontato la propria esperienza:

Per raggiungere $\mathrm{i}$ traguardi che mi ero prefissata ho trovato fondamentale l'accompagnamento che ho ricevuto da docenti che hanno creduto nelle mie possibilità. Vorrei che un giorno un ragazzo si ricordasse di me come io ricordo il mio maestro delle elementari, che durante le vacanze estive mi ha aiutato a migliorare il mio italiano. Oppure la docente della commissione dell'esame di maturità, la quale, dopo aver letto il mio tema sull'integrazione dei ragazzi stranieri, mi ha incoraggiata e mi ha invitata a realizzare ciò che avevo scritto. Oppure altri docenti che, ogni volta che incontrano me e i miei fratelli, sono orgogliosi di noi e questo orgoglio che ci hanno sempre mostrato è stata la forza che ci ha permesso di realizzare la maggior parte dei nostri sogni. (Dal diario di bordo di D.)

Dal punto di vista educativo, la resilienza implica la capacità di superare gli ostacoli che ogni processo di apprendimento presenta. Gli studi della psicologa C. Dweck (2000) sembrano dimostrare che il grado di resilienza degli studenti dipende in modo rilevante dalla capacità dei docenti di aiutarli a metabolizzare e a superare le frustrazioni presenti nel percorso di formazione e di sostenerli nel "contenimento" delle loro ansie. 


\section{Tutori di resilienza}

Il titolo "Almeno una stella" richiama direttamente le osservazioni di B. Cyrulnik, confermate anche da altri studiosi, sull'importanza vitale, come fattore di costruzione di resilienza, di almeno una persona, interna o esterna alla famiglia, che funga da "stella" per chi, come gli adolescenti immigrati, sperimenta situazioni di particolare difficoltà.

La stella, nel nostro caso, è rappresentata simbolicamente dal tutor, che si spende anche personalmente nel rapporto con i ragazzi migranti e ne sostiene il cammino, intrecciando con loro una relazione significativa. L'esperienza di due anni di lavoro ha dimostrato che, spesso, le stelle hanno una funzione di moltiplicatore, perché sono in grado di attivare il contesto scolastico e/o extrascolastico, a sostegno dei tutorati. Il lavoro dei tutor ha lo scopo di mettere in atto un processo di empowerment, inteso come ampliamento delle possibilità di agire e di scegliere di un individuo nel proprio contesto, per riappropriarsi della capacità decisionale e non delegare ad altri la soluzione dei propri problemi.

I tutori di resilienza, secondo Cyrulnik (2000), sono persone che devono saper prendere su di sé il dolore dei minori che accompagnano e dargli un significato; figure adulte di riferimento che Malaguti (2005) connota come "adulti solidi, presenti, responsabili". I tutor di "Almeno una stella" sono indirizzati verso un ruolo del genere, ma ognuno lo svolge secondo le proprie capacità, l'età, l'esperienza maturata. Graziella Favaro (2013) li descrive come "sostegno, guida, una sorta di fratello maggiore" ... "che ha contribuito ad accogliere, almeno in parte, la vulnerabilità degli adolescenti stranieri".

Il tutor non è uno psicologo e il suo impegno ha limiti evidenti, ma egli porta se stesso, la propria empatia, la giovane età e, spesso, il fatto di aver vissuto vicende analoghe, al servizio dei ragazzi che accompagna. Queste qualità sono state essenziali, perché il ruolo del tutor, in Trentino, è stato indirizzato prevalentemente in senso relazionale, in particolare con i ragazzi delle scuole superiori, con cui si è privilegiato l'aspetto dell'accompagnamento sul territorio. I tutor hanno rappresentato uno tra i fattori protettivi che aiutano nella costruzione e nell'avvio di processi positivi.

In alcuni casi i tutor hanno agito concretamente per migliorare l'integrazione degli studenti sul territorio e per dare loro strumenti utili a muoversi nel sociale.

A. è un ragazzo senegalese, socievole e solare, emigrato qui da pochi mesi solo con il padre, che soffriva la solitudine. Data la spiccata passione e il talento naturale per il calcio, e grazie al suggerimento delle responsabili del progetto, l'ho aiutato a iscriversi alla squadra locale dei ragazzi della sua età. Per farlo, abbiamo impiegato alcuni mesi. (Dal diario di bordo di G.) 
Essere attivo in un'organizzazione sportiva locale, dove è stato introdotto da un amico, è stato per E. un supporto sociale importante, che lo ha aiutato, nei primi mesi qui, a recuperare fiducia e speranza. Verso la fine del percorso di tutoring, ha espresso il desiderio di trovare un lavoro stagionale nel settore alberghiero e mi ha chiesto aiuto. Assieme abbiamo lavorato a un curriculum, poi l'ho accompagnato, presentandolo, in alcuni alberghi della zona - lui era molto intimidito - e siamo rimasti d'accordo che sarebbe tornato, senza di me, a portare il curriculum, non appena fosse stato completato. È stato molto contento, perché da solo non avrebbe saputo come muoversi. (Dal diario di bordo di A.)

L'importanza del tutor sta soprattutto nel saper ascoltare senza giudizio le confidenze dei ragazzi, nel costituire un modello positivo per esperienze e percorsi fatti (molti dei tutor sono stati scelti anche per i loro vissuti di sradicamento e migrazione), nel promuovere l'acquisizione di fiducia in se stessi e la consapevolezza delle proprie capacità, riconoscendo e mettendo in luce potenzialità e ricchezze, come il bilinguismo, difficilmente apprezzate nel sociale e nell'ambito scolastico.

Il trovarsi a fianco di E. non come insegnante o amica, ma come persona disposta ad ascoltare, è stata, credo, l'essenza del percorso compiuto. (Dal diario di bordo di A.)

Il tutor ha rappresentato, nei limiti delle sue possibilità, una presenza affettiva stabile. Per le ragazze e i ragazzi più riservati e silenziosi, che mostravano pudore nell'esprimersi e nel parlare di sé, è stato decisivo che il tutoring sia durato due anni (cosa che, purtroppo, non è potuta avvenuta per tutti).

Alla fine di quest'esperienza di due anni con I. e T., posso dire che si è creato con le ragazze un forte legame. Prima di quest'anno I. non si era mai lasciata abbracciare, mentre oggi ci salutiamo come due amiche. (Dal diario di bordo di C.)

In ogni caso, la conquista della fiducia non è mai immediata, come confermano i brani tratti dai diari di bordo di due tutor.

Certo, non è sempre stato un percorso agevole: conquistare la fiducia, e in certi casi abbattere la diffidenza, di ragazzi che non sempre avevano scelto il percorso consapevolmente; ci è voluto del tempo... (Dal diario di bordo di T.)

A. si è dimostrato, incontro dopo incontro, sempre più aperto. Le prime volte era taciturno; pensavo fosse un problema linguistico, in realtà era necessario del tempo perché si creasse un rapporto di fiducia... (Dal diario di bordo di G.) 
Aiutare i giovani che si accompagnano a immaginarsi un futuro e a delinearne le tappe rientra tra gli impegni dei tutori di resilienza. Soprattutto alcune tra le tutor hanno saputo divenire canale di ascolto e andare a fondo delle aspirazioni e dei sogni confidati loro, che avevano bisogno di essere dipanati e incoraggiati.

N. era desiderosa di approfondire soprattutto i temi della prospettiva futura di un lavoro e della possibilità di studiare anche dopo le superiori e reagiva con determinazione alla difficoltà di non sapere bene l'italiano e di non avere avuto una regolare formazione scolastica. Sostenendo e stimolando alcune immagini di come vede il suo futuro, si è arrivati, con lei, all'aiuto verso gli altri. (Dal diario di bordo di A.)

Anche le azioni di ri-orientamento di alcuni adolescenti, che avevano confidato ai tutor dubbi e predilezioni, hanno avuto lo scopo di rendere possibile la costruzione di un progetto a medio termine e di curare le prospettive per il futuro, coinvolgendo anche la famiglia. Alcune tutor hanno seguito, d'intesa con lo staff di progetto, gran parte del percorso, accompagnando ragazze e ragazzi a visitare le realtà scolastiche cui erano interessati e ai colloqui informativi organizzati per loro.

Y. non mostra preoccupazione per il superamento dell'anno, ma vuole cambiare scuola perché non crede di avere possibilità di successo nel portare avanti il percorso di ragioniere, per le gravi lacune in matematica. Per questo vede nella scuola alberghiera una chance per il suo futuro, anche se la motivazione non è chiara. L'ho accompagnato a due incontri all'Istituto alberghiero; nel secondo, con il vicedirettore, è stata presentata l'istanza di iscrizione e successivamente abbiamo reperito il materiale da studiare per il colloquio di sbarramento. (Dal diario di bordo di S.)

Z. dice di essere capitato all'Istituto tecnico per sbaglio, ovvero di aver perso la scadenza dell'iscrizione al Centro professionale e di essere stato costretto a ripiegare su qualcos'altro, senza esserne troppo consapevole. Mi dice che vuole fare l'elettricista, che ha bisogno di fare cose pratiche e manuali. Gli spiego che anche nelle scuole professionali ci sono materie teoriche da studiare. Z. si dimostra subito molto interessato e insieme leggiamo il sito del Centro professionale e i programmi dei vari percorsi formativi. Qualche settimana dopo andiamo a visitare la scuola e ci mostrano le officine. Z. è affascinato. Seguo poi lui e la famiglia nei passi necessari al ri-orientamento. A volte ho avuto la sensazione che, se non ci fosse stato questo percorso, Z. e la sua famiglia non avrebbero fatto nulla per cambiare la situazione di disagio che viveva a scuola, anche se era tale da indurlo a comportamenti trasgressivi per lui inconsueti. (Dal diario di bordo di S.) 


\section{Autostima e ri-motivazione}

Al tutor era affidato anche il compito di sostenere e incoraggiare gli studenti nel processo di apprendimento. Il sostegno scolastico è stato inteso, in generale, come rafforzamento della motivazione allo studio e poi, secondo i casi, come aiuto nello svolgimento dei compiti e nella preparazione delle interrogazioni, supporto nell'italiano per lo studio (spiegazione di termini tecnici o ricercati e loro "traduzione" in un linguaggio più comprensibile) e nella produzione scritta in italiano (contributi alla stesura di elaborati scritti tramite tracce, schemi, ecc.), assistenza nella selezione e nell'analisi dei materiali utili per ricerche in biblioteca o sul web, ecc.

Ho puntato molto sulla fase di ricerca dei materiali perché A. mi ha confessato che in Grecia non aveva mai dovuto fare una ricerca su internet, mentre quest'aspetto, per la scuola italiana, è molto importante. (Dal diario di bordo di D.)

Il lavoro dei tutor, assieme alle azioni intraprese dal gruppo di progetto, ha favorito il concreto realizzarsi di percorsi di studio personalizzati per i ragazzi migranti, come richiesto anche dalla normativa (Linee guida nazionali del 2011 e provinciali del 2012). L'attivazione di una didattica mirata può consentire a studenti in difficoltà di mettere a frutto con successo le loro risorse. Essere guidati in percorsi e prove commisurati alle proprie capacità significa fare esperienze che aumentano la fiducia in se stessi e la consapevolezza di poter conseguire esiti positivi. Significa anche intraprendere un cammino che può contribuire al processo di costruzione della resilienza. Alla fine dell'anno scolastico una docente, dopo più incontri con la tutor e il gruppo di lavoro, si è assunta spontaneamente il compito d'incontrare, durante l'estate, una studentessa respinta, per monitorarne il percorso di apprendimento estivo, assegnato per consentirle di frequentare con esito positivo l'anno successivo.

La collaborazione tra tutor, équipe del progetto e docenti ha consentito che il punto di vista dei tutor, il loro sguardo positivo e le loro informazioni "privilegiate" su risorse presenti negli adolescenti stranieri contaminassero, in molti casi, la visione degli insegnanti, più attenti alle carenze e "ai vuoti" piuttosto che "ai pieni".

Studiando con A. per il tema di epica e leggendo nel testo la spiegazione dei diversi generi epici, tra cui quello bretone e carolingio, mi sono resa conto della disparità tra le conoscenze scolastiche e culturali di uno studente che ha studiato in Italia e di uno che entra in una scuola italiana a 16 anni. A. conosce bene l'epica greca, ma nessuna delle altre. (Dal diario di bordo di D.)

Alcuni maschi adolescenti di seconda generazione hanno mostrato grande fragilità e messo in atto comportamenti che li hanno esposti al rischio 
d'insuccesso e abbandono scolastico. Le loro provocazioni spesso nascono dal fatto che non sono in grado di controllare le proprie emozioni, uno dei fattori necessari per educarsi alla resilienza. Il confronto con loro è stato per i tutor un'esperienza complessa e impegnativa, non esente da rischi, che talvolta non ha dato i risultati sperati per svariati motivi, tra cui la durata del tutoring, limitata se posta in relazione all'ampiezza e all'urgenza dei bisogni di questi adolescenti. Soprattutto è difficile, e spesso inattuabile, attivare una rete di persone con competenze diverse, che si facciano carico dei vari aspetti del problema. Questi ragazzi mettono a dura prova anche gli insegnanti, che vengono continuamente sfidati, hanno grandi difficoltà a entrare in comunicazione con loro e a mobilitarne le risorse e spesso esprimono giudizi definitivi e inappellabili in merito alle loro possibilità di successo. La presenza di un singolo tutor non è sufficiente a risolvere la situazione, ma, in qualche caso, il lavoro congiunto di tutor e gruppo di progetto ha evitato l'espulsione dal percorso formativo.

S. dice che non gli importa della scuola, ma in realtà è evidente che questo ritardo scolastico lo fa soffrire: vive la scuola con ansia, come un obbligo al quale non vuole sottostare. Vive un forte conflitto in famiglia, specialmente con il padre, molto rigido, che talvolta ha reazioni esagerate nei suoi confronti, che lo spingono ad allontanarsi dalla famiglia per cercare conforto e protezione nel gruppo dei pari. I genitori e gli insegnanti, e l'adulto in generale, fungono sempre da antagonisti. S. vive ogni richiesta scolastica e non, anche quelle legate al progetto che stiamo portando avanti assieme, come un tentativo di prevaricazione. Spesso mi dice che lui non si fa mettere i piedi in testa da nessuno. Per lui il rispetto si guadagna prevaricando gli altri, perché, dice, - in Marocco funziona così -. Però, quando si sente ferito, reagisce con una rabbia talvolta così sproporzionata, da far pensare a un dolore per lui intollerabile. (Dal diario di bordo di M.)

Ogni cosa che gli viene proposta, Y. la vive come un attacco personale e quindi in qualche modo si ribella, come quando, pur di non accontentare la prof. di matematica, si è rifiutato di comprare un quaderno degli esercizi come lo voleva lei. Y. fatica ad accettarsi e a farsi aiutare. È negativo in tutto. Anche quando gli viene riconosciuto qualcosa, si sminuisce da solo. Cerca la mia approvazione quando mi racconta, senza troppi dettagli, le bravate del sabato sera. Mi chiede di me, cosa faccio, come affronto le difficoltà, che musica ascolto, come faccio a rapportarmi con le persone, come se stesse per forza cercando un modello cui aggrapparsi, nonostante la differenza di genere. Dichiara che l'unica cosa che gli interessa è allenarsi in palestra la sera; l'idea di poter modificare il suo corpo attraverso l'alimentazione e l'allenamento lo esalta. Col passare degli incontri mi accorgo che Y. ha bisogno di sentire che qualcuno si fida di lui, che gli voglia bene sinceramente e che per questo lo accetti per quello che è, senza giudicarlo. (Dal diario di bordo di S.) 


\section{Alcune riflessioni dopo l'esperienza}

Le esperienze fatte hanno convalidato alcune ipotesi alla base del progetto, che aveva lo scopo di sperimentare la figura del tutor nell'accompagnamento e nella tutela di giovani adolescenti migranti in difficoltà e di avviare azioni di educazione alla resilienza. Alcune di queste sembrano meritevoli di essere segnalate.

Per quanto riguarda il rapporto tra ragazzi e tutor, si è rivelato un valido strumento di aiuto il dare priorità alla cura della relazione e degli aspetti emotivi e all'integrazione degli adolescenti migranti, accompagnandoli nella conoscenza delle opportunità aggregative offerte dal territorio, e mettendo in secondo piano il sostegno scolastico. Questa strategia ha permesso loro di superare momenti transitori di disagio e ha creato relazioni che, in qualche caso, si sono mantenute anche oltre la conclusione del tutoring.

Il legame che si è creato con le ragazze va ben oltre l'aiuto nello studio: si è creato un legame di affetto fraterno. Anche se il progetto è finito e le nostre vite prenderanno strade diverse, ci siamo ripromesse di tenerci in contatto con messaggi e telefonate e di vederci ogni tanto. (Dal diario di bordo di C.)

L'uso del tutoring come momento di ascolto attivo ha consentito di superare i confini del sostegno scolastico e di accogliere confidenze, pensieri e progetti di ragazzi e ragazze, volti a delinearne il futuro e a individuare le scelte intermedie per costruirlo, dando seguito ai propri sogni.

L'impegno dei tutor e delle tutor è stato un esempio di assunzione di responsabilità nel sociale e nei confronti di soggetti deboli, da cui i giovani migranti potevano apprendere. Parecchi tutor si sono dovuti cimentare in una sorta di educazione alla cittadinanza, al rispetto dell'altro e degli impegni presi, con gli adolescenti loro affidati, che arrivavano spesso in ritardo o non si presentavano affatto agli incontri, senza neppure avvisare, e che avevano un atteggiamento analogo nei confronti della frequenza scolastica.

Mi dispiace di non essere riuscita del tutto a far capire alle ragazze l'importanza della scuola e dell'impegno, anche nella vita, della costanza e dell'essere sempre presenti e puntuali. (Dal diario di bordo di C.)

Y. agli incontri arriva sempre dai 10 ai 20 minuti in ritardo, senza avvisare e nonostante i miei continui messaggi per ricordargli gli appuntamenti e i richiami alla puntualità, nonché al rispetto nei miei confronti. Nonostante ciò Y. non è mai puntuale, addirittura dimentica gli incontri e racconta, con tranquillità, di arrivare in ritardo anche a scuola, motivo per cui spesso salta temi e interrogazioni. (Dal diario di bordo di S.) 
Sembra che Z. viva in Marocco quando è a casa e in Italia quando è a scuola e che, per questo, il momento della scuola possa in qualche modo essere più trasgressivo. (Dal diario di bordo di S.)

I tutor sono stati arricchiti dall'esperienza e dal rapporto con ragazzi e ragazze migranti e le competenze maturate, per alcuni di loro, potrebbero essere giocate anche sul piano professionale. Tutti hanno espresso soddisfazione per aver partecipato al progetto.

L'incontro con loro mi ha sempre profondamente arricchito: lo scambio di storie, l'ascolto dei loro vissuti, spesso difficili e dolorosi, mi hanno donato più di quanto io potessi offrire loro in termini d'insegnamento. La possibilità che mi è stata data dal progetto "Almeno una stella" è stata, per me, estremamente preziosa. M'incuriosiva l'adozione dello strumento del tutoring nella costruzione di un rapporto educativo in ambiente scolastico e sociale. Inoltre costruire questo percorso assieme a un adolescente, in una fase della vita delicata in cui si snodano la costruzione dell'identità e la scoperta del sé, ha rappresentato una bella sfida educativa, anche dal punto di vista professionale. (Dal diario di bordo di A.)

Per quanto riguarda la scuola, la sinergia con i tutor può aiutare i docenti ad avere uno sguardo più ampio e con meno pregiudizi, in un'ottica di prevenzione del disagio sia per gli adolescenti stranieri, sia per gli insegnanti, cui spesso mancano gli strumenti e il tempo per comprendere le difficoltà, i comportamenti e le reazioni degli studenti loro affidati.

Sperimentare lo strumento del tutoring a scuola è quindi importante per perfezionarlo e studiare il modo migliore perché possa diventare momento di collaborazione e stimolo per i docenti.

La restituzione da parte dei tutor alle scuole in merito alla valorizzazione dei ragazzi migranti e delle loro risorse, che i docenti non sempre riescono a far emergere, può costituire un momento di scambio e di arricchimento in cui prospettive diverse si confrontano. I tutor potrebbero stimolare, con il loro sguardo mirato e "più libero", la costruzione di percorsi educativi e didattici in cui le esigenze dei processi di apprendimento possano venire a patti con i punti di vista, le esperienze e i bisogni degli adolescenti di origine migrante.

\section{Riferimenti bibliografici}

Cyrulnik B. (2000), Il dolore meraviglioso, Frassinelli, Milano.

Cyrulnik B. (2003), Il coraggio di crescere: gli adolescenti e la ricerca della propria identità, Frassinelli, Milano.

Cyrulnik B. (2007), La vulnerabilità come risorsa per essere felici, Frassinelli, Milano. 
Cyrulnik B. (2009), Autobiografia di uno spaventapasseri, Raffaello Cortina, Milano.

Cyrulnik B., Malaguti E. (a cura di) (2005), Costruire la resilienza, Erickson, Trento.

Dweck C.S. (2000), Teorie del sé. Intelligenza, motivazione, personalità e sviluppo, Erickson, Trento.

Marcoli A. (2003), Passaggi di vita. Le crisi che ci spingono a crescere, Mondadori, Milano.

Malaguti E. (2005), Educarsi alla resilienza, Erickson, Trento.

Novara D. (2011), La grammatica dei conflitti, Sonda, Casale Monferrato. 


\section{I diversi stili dei tutor \\ di Francesca Terenzi*}

\section{Gli stili e le strategie di apprendimento}

La presente riflessione nasce dal bisogno semplice e allo stesso tempo complesso di rispondere a questi quesiti: in che modo le persone sono diverse quando imparano? Queste diversità condizionano i processi di apprendimento e di insegnamento? Che cosa implica riconoscere e valorizzare le diversità? Fino a che punto è possibile "individualizzare" l'apprendimento? Quale "gamma" di opportunità di intervento è possibile ipotizzare per "gestire nel quotidiano" queste differenze?

Una definizione data da Keefe (1979) può costituire un utile punto di partenza:

Gli stili di apprendimento sono caratteristici comportamenti cognitivi, affettivi e fisiologici che funzionano come indicatori relativamente stabili di come i discenti percepiscono l'ambiente di apprendimento, interagiscono con esso e vi reagiscono (Keefe, 1979).

In questa affermazione sono qui delineate alcune idee-chiave:

- la dimensione comportamentale: lo stile di apprendimento non è solo un costrutto teorico, ma, in quanto si manifesta in concreti comportamenti, funziona come indicatore, cioè come un segnale, di caratteristiche più "nascoste" della persona;

- la globalità del concetto: nonostante l'enfasi talvolta posta sugli aspetti cognitivi dell'apprendimento, uno stile è un tipico modo di manifestare la propria individualità anche fisica e socio-affettiva;

- la relativa stabilità: trattandosi del riflesso della propria personalità, lo stile è stabile tanto quanto la personalità che esprime: soggetto dunque a cambiamenti ed evoluzioni, e particolarmente in età evolutiva, ma con una base che è parte costitutiva dell'individualità della persona;

- la funzione di "filtro": lo stile, insieme a fattori quali convinzioni, atteggiamenti e motivazioni, dai quali è difficilmente separabile, agisce come "filtro" rispetto a come viene percepito l'ambiente di apprendimento: i "compiti" a cui abbiamo accennato, ad esempio, una volta scelti dall'insegnante, vengono reinterpretati da ciascuno studente in modo diverso a seconda degli "occhiali interpretativi" costituiti dal suo stile;

- l'interazione e la reazione con l'ambiente: lo stile condiziona anche il modo in cui la persona si rapporta all'ambiente di apprendimento, che comprende tutti i fattori del relativo contesto (dall'insegnante ai compagni, dai libri

\footnotetext{
* Oxfam Arezzo e Centro di Documentazione Città di Arezzo.
} 
di testo alle procedure didattiche, dagli strumenti utilizzati alle modalità di valutazione...).

Gli stili di apprendimento rappresentano quindi le modalità preferenziali secondo cui i diversi individui apprendono; i termini più specifici possono essere considerati come "la predisposizione ad adottare una particolare strategia di apprendimento indipendentemente dalle caratteristiche specifiche del compito".

Quanto alla strategia di apprendimento - concetto che a questo punto diviene basilare per intendere quello di stile -, questa si può definire come un insieme di procedure utilizzabili per conseguire l'apprendimento.

Diventare più strategici nel proprio apprendimento significa attivare operazioni di varia natura costantemente in interazione. Le principali gamme di strategie sono riconducibili a:

- le strategie cognitive: riguardano l'elaborazione delle informazioni, cioè l'appropriazione dei contenuti informativi all'interno della propria struttura. Queste strategie sono all'interno di operazioni mentali complesse, come l'associazione (ad esempio, richiamare le proprie conoscenze di un argomento prima di leggere un testo), la classificazione (come raggruppare le informazioni di un testo per categorie), l'inferenza/deduzione (ad esempio, utilizzare indizi linguistici ed extralinguistici per ipotizzare significati), l'induzione (come risalire da elementi particolari alla formulazione di una regola generale), il trasferimento (ad esempio, collegare quanto appreso con la propria realtà quotidiana o con i contenuti di altre aree disciplinari);

- le strategie meta cognitive: agiscono come "bussole" più generali, in quanto controllano e orientano le operazioni complessive di auto-gestione del proprio apprendimento. È così che funzionano le strategie di pianificazione (ad esempio, stabilire obiettivi a breve, medio e lungo termine, programmate le condizioni fisico-ambientali dello studio, chiarire le richieste e i vincoli dei compiti da svolgere), le strategie di controllo (come identificare problemi nella lettura, ricercare le possibili cause dei problemi e ipotizzare possibili soluzioni) e le strategie di autovalutazione (ad esempio, confrontare gli obiettivi attesi con i risultati ottenuti, analizzare i propri errori, verificare l'utilità delle strategie utilizzate);

- le strategie socio-affettive aiutano a gestire il proprio rapporto con se stessi (ad esempio, ricorrere a tecniche per ridurre lo stress, premiarsi per un compito ben eseguito, tenere un diario per registrare le proprie emozioni) e con gli altri, genitori, insegnanti, compagni, con cui si condivide l'esperienza dell'apprendimento (ad esempio, ricorrere all'aiuto di amici o di altre figure di sostegno, tenere sotto controllo i propri interventi in un lavoro di gruppo, chiedere e accettare di essere corretti e di ricevere un feedback da altri sulle proprie prestazioni);

- le strategie motivazionali, che possiedono forti connotazioni affettive, sono rivolte a promuovere non solo la spinta motivazionale iniziale, ma anche, e forse soprattutto, ad aiutare la persona a persistere nell'impegno una volta 
iniziata il compito. Per esempio controllare le distrazioni presenti nel proprio ambiente di studio, crearsi immagini positive di se stessi prefigurando un successo invece che un fallimento, concentrarsi sul compito senza guardare continuamente l'orologio...

\section{La relazione tutor/tutorato nello studio}

Gli studi di educazione interculturale (Demetrio, Favaro, 2002; Gobbo, 2001; Nanni, 2001; Perotti, 1994) e le numerose esperienze svolte all'interno di percorsi formativi individuano come approccio da privilegiare alle discipline quello di tipo olistico.

Intendiamo con questo sottolineare l'importanza dell'uso della contestualizzazione culturale relativamente ai contenuti, dell'attenzione alla continua negoziazione dei significati da parte dell'insegnante che si fa mediatore interculturale tra l'allievo e il sapere disciplinare, dell'utilizzo di strategie e tecniche didattiche varie e integrate che coinvolgano tutti e cinque i sensi e del ricorrere alla cooperazione tra pari e alle risorse di tutti da giocare in gruppo.

In questo modo si possono offrire pari opportunità agli allievi per entrare in relazione positiva con il sapere, anche in un contesto estraneo alla cultura d'appartenenza.

Se gli insegnanti conoscono le culture d'appartenenza dei loro studenti, possono anche comprendere le diversità degli stili di apprendimento, anche se è fondamentale non incorrere nel rischio di pensare che individui con stili di apprendimento simili appartengano necessariamente alla stessa cultura.

Inoltre, è necessario puntare sulla consapevolezza reciproca degli stili di apprendimento tra allievo e allievo/allievo e insegnante e degli stili di insegnamento tra insegnante e allievo, per evitare che il cambiamento da una cultura all'altra, da una scuola all'altra, da una realtà all'altra, provochi a catena, da parte degli allievi stranieri, atteggiamenti di estraneità, di isolamento e di frattura nei confronti della scuola. Solo esplicitando lo stile d'insegnamento del docente e facendo emergere gli stili di apprendimento degli studenti si può pensare che venga intessuto tra essi un reale rapporto di scambio e reciprocità.

Al fine di organizzare il percorso di insegnamento/apprendimento tenendo conto delle differenze individuali e della valorizzazione dei talenti e delle esperienze pregresse, il ruolo dell'insegnante all'interno del laboratorio, e dei tutor stessi, diventa quello di facilitatore in grado di attivare un tipo di apprendimento meno passivo, rispetto a quello svolto abitualmente in classe.

Diventa fondamentale, quindi, creare nel gruppo un clima positivo, di fiducia e di valorizzazione e sviluppo dei talenti individuali e della sfera affettiva, cercando di valorizzare i diversi stili di apprendimento. 
Il tutoring pone due obiettivi educativi primari: imparare ad imparare e imparare a lavorare con gli altri.

L'adulto o il coetaneo con maggiore esperienza "offrono" il suo modello di problem solving non nel contenuto, ma nella strategia per arrivare alla soluzione di problemi; i coetanei sono più efficaci nei processi di apprendimento perché offrono modelli di problem solving più semplici e più vicini a chi deve apprenderli.

Il tutoring prevede un passaggio di competenze tra tutor e tutor e questo presuppone una consapevolezza delle strategie utilizzate per acquisire informazioni e risolvere problemi.

Come può il tutor insegnare, facilitare, suggerire le strategie?

Lo studente deve avere un ruolo attivo nel costruire le proprie conoscenze metacognitive. Il tutor promuove le conoscenze metacognitive attraverso modeling, dialogo e discussione. "Che cosa stai facendo esattamente?" o "Perché lo stai facendo?" o "Come ti aiuta?" o "Che cosa farai con il risultato?"

In secondo luogo il tutoring ha un notevole impatto sugli aspetti sociali; imparare a lavorare con gli altri significa aiutarsi e sostenersi reciprocamente, apprezzare maggiormente i propri compagni, si considerano come amici che aiutano nel processo di apprendimento.

I tutorati hanno la possibilità di confrontarsi con un modello proposto dai tutor fatto di collaborazione e di scambi, di aiuto reciproco laddove le competenze di uno non arrivano, ma anche di amicizia e di solidarietà.

\section{Le "4 domande" ai tutor}

Nella seconda parte del percorso di tutoring, il gruppo è stato sollecitato a riflettere in maniera più strutturata sul proprio intervento; questa riflessione è stata poi utile anche per la compilazione del diario del tutor (soprattutto per la parte "riflessioni finali sulla mia esperienza").

Le domande sono state poste in maniera scritta e rivolte a tutti i tutor; tutti hanno risposto. Fra queste le prime due hanno fornito vari spunti di approfondimento.

1. Quale è stato il tuo approccio didattico e relazionale con i ragazzi?

2. Quali sono stati i tuoi punti di forza e i tuoi punti di debolezza?

3. Nel caso tu abbia seguito più ragazzi, pensi che la tua relazione sia stata sempre la stessa con tutti? Come hanno risposto ai tuoi stimoli?

4. Trova un immagine che ti rappresenti e spiega i motivi facendo anche degli esempi. 
Domanda 1. Quale è stato il tuo approccio didattico e relazionale con i ragazzi?

Questa domanda riprende nella prima parte un lavoro di riflessione che ha seguito quasi tutto il percorso in classe; spesso, infatti, davanti alle varie difficoltà o dubbi riportati dai tutor stessi abbiamo cercato di riflettere insieme sul tipo di atteggiamento e sulle risposte che venivano portati avanti.

Per esempio ripotiamo alcune domande ricorrenti in ordine temporale: "Come posso spiegare un termine o un concetto che il tutee non comprende?", "Come posso aiutarlo ad organizzarsi nello studio?", "Come posso favorire la sua concentrazione?".

Insegnare e imparare nella nuova lingua non è semplice; specialmente se non si è vissuta in prima persona questa esperienza risulta difficile capire quali strategie didattiche assumere.

Per affrontare meglio questo aspetto, oltre alla formazione specifica sul tema dell'insegnamento e dell'apprendimento della L2, è stato predisposto e condiviso insieme al gruppo una sorta di prontuario delineando le modalità comunicative e le strategie fondamentali:

Per la morfologia e la sintassi

- usare frasi il più possibile brevi, semplici e regolari (soggetto-verbocomplemento oggetto)

- esplicitare sempre il soggetto

- usare i verbi al presente

- utilizzare preferibilmente la coordinazione, usando il meno possibile congiunzioni subordinanti

- utilizzare preferibilmente il modo indicativo

- utilizzare preferibilmente la forma affermativa evitando quella negativa

- usare frequentemente le ripetizioni

Per il lessico

- usare un lessico non troppo vasto, ma di alta frequenza

- spiegare i concetti specifici introdotti con parole di uso comune

- non usare espressioni idiomatiche, incomprensibili a chi non appartiene alla cultura in cui sono nate

- preferire la forma concreta a quella astratta, non visibile o contestualizzabile

- limitare l'uso dei pronomi e sinonimi

- non usare sinonimi per spiegare termini non compresi dall'alunno

- non usare figure retoriche

Criteri di scrittura controllata per la semplificazione testuale

- evidenziare i concetti fondamentali direttamente sul testo

- organizzare l'idea principale e i concetti chiave in uno schema logico-sequenziale semplice chiaro 
- riscrivere il testo semplificato

- ridurre in modo drastico la parte scritta inserendo molte illustrazioni e disegni

- usare un carattere grande e chiaro senza modificarlo più volte

- usare molte immagini ed esempi

Per quanto riguarda le altre due domande legate maggiormente alle difficoltà di "insegnare ad imparare" sono state proposte alcune attività ludiche e di simulazione per poi riflettere in gruppo sulle varie risposte.

\section{Attività 1 - Partiamo per il nostro viaggio}

Qual è la prima cosa da fare quando si vuole partire per un viaggio?

Le valigie!!!

Di cosa ho bisogno?

Cosa ho in casa e cosa devo comprare?

Fare le valigie significa organizzarci

Pensa ad una attività che devi fare (studiare un capitolo di storia, fare una ricerca, andare a danza...)

Organizza questa attività secondo le seguenti domande:

Tempo: quanto tempo mi ci vuole per...?

Materiali: che cosa mi occorre per...?

\section{Attività 2 - Organizziamo il nostro studio}

Segna con una $\mathrm{x}$ la risposta che si avvicina maggiormente al tuo metodo

Cosa faccio quando studio?

Prima di leggere il testo:

$\square$ leggo i titoli dei paragrafi e le parole in grassetto

$\square$ guardo le figure e i grafici

$\square$ cerco il nocciolo del discorso trasformando i titoli in domande

Mi domando:

$\square$ che cosa so sull'argomento?

$\square$ ricordo la spiegazione dell'insegnante?

$\square$ ho visto film o letto libri/riviste/articoli sull'argomento? 
Quando leggo:

$\square$ leggo attentamente il testo cercando di capire

$\square$ tengo a mente lo scopo e l'argomento per cui sto leggendo

$\square$ mi fermo ogni tanto per capire se sto capendo

$\square$ attingo alle mie conoscenze in L1

Quando sottolineo:

$\square$ cerco le frasi che si riferiscono al titolo

$\square$ sottolineo con colori diversi in base ai diversi temi/argomenti

Quando ripeto:

$\square$ ripeto quando ho letto tutto

$\square$ ripeto spezzettando il testo

$\square$ rispondo alle mie domande o alle domande degli esercizi

$\square$ formulo un discorso completo

Alla fine:

$\square$ ripeto paragrafo per paragrafo

$\square$ ripeto le frasi sottolineate

$\square$ ripeto l'intero capitolo

La riflessione sul modello di relazione instaurata tra tutor e tutorato è stata proposta attraverso l'analisi dello strumento "Carta T". A partire da un esempio di carta (abilità: incoraggiare) il gruppo ha riflettuto su varie abilità indispensabili nella relazione di aiuto allo studio con studenti in particolare di origine straniera (ascolto attivo, cooperazione...).

Costruire una "Carta $\mathrm{T}$ " significa quindi preparare una tabella sulla quale nella parte alta viene indicata l'abilità che si intende insegnare, definita attraverso i comportamenti verbali e non verbali che la descrivono. Ad esempio, sono state utilizzate delle domande stimolo: "Cosa vedo quando sono in presenza di una persona che utilizza l'abilità di ascoltare?" "Cosa sentono le mie orecchie quando sono in presenza di una persona che utilizza l'abilità dell'ascolto?" 
Abilità

Cosa vedo con gli occhi

(Comportamento non verbale)
Cosa sento con le mie orecchie

(Comportamento verbale)

Per incoraggiare

sguardo di assenso

annuire con la testa

dare un colpetto sulla spalla

battere le mani
"Ti sei espresso proprio bene"

"Hai fatto fatica ma alla fine ci sei riuscito"

"Forza, continua così..."

"È stato molto di più quello che hai fatto correttamente di quello che hai sbagliato"

L'approfondimento e la riflessione sul tema della relazione sono stati sicuramente più sentiti e più vicini all'esperienza dei tutor, dei quali riportiamo alcuni passaggi tratti dalle risposte alla prima domanda.

"Ho cercato di sostenere la motivazione e la voglia di riuscita e allo stesso tempo di stabilire una relazione di fiducia".

"Il mio approccio è stato amichevole, ho cercato di far sentire $i$ ragazzi al loro agio senza dare l'impressione che si trovino davanti a un insegnante, ma in compagnia di un'amica".

\section{Punti di forza e di debolezza}

Domanda 2. Quali sono stati i tuoi punti di forza e i tuoi punti di debolezza come tutor?

Dall'analisi delle risposte dei tutor alla domanda 2 si registrano i seguenti punti di forza e di debolezza.

Punti di forza

- Rendere lo studio meno pesante

- Essere competente nella disciplina affrontata

- Riuscire a mantenere alto il livello di attenzione

- Entrare in sintonia con lo studente

- Creare un clima di complicità

- Creare un ambiente di fiducia dentro il quale lo studente si senta libero di sbagliare

- La pazienza e la costanza affettiva

- Approcciarsi con il sorriso e con positività 


\section{Punti di debolezza}

- Facilitare lo studio delle discipline soprattutto per quanto concerne la lingua italiana utilizzata

- Coinvolgere lo studente nello studio della disciplina

- Comprendere la reale difficoltà dello studente straniero

- Non riuscire sempre a distinguere bene la linea che separa il bisogno di rassicurazione per non accrescere la sfiducia e il bisogno di stimoli per non fermarsi

- Non essere autorevoli, ma avere autorevolezza

I punti di forza possono essere raggruppati in due insiemi diversi: competenze legate allo studio delle discipline e dell'italiano e capacità di relazione.

Solo una minima parte dei punti elencati si riferisce al primo insieme, giudicando importante il possesso di competenze scolastiche e la relativa capacità di stimolare lo studio senza appesantirlo.

Questo deriva in parte dell'organizzazione del laboratorio stesso; infatti in un primo momento le coppie tutor/tutorato o i piccoli gruppi si sono formati mettendo in relazione le competenze scolastiche dei tutor con le maggiori difficoltà riportate dagli studenti stessi. Solo in alcuni casi la coppia si è formata per vicinanza linguistica e culturale.

I tutor si sono poi dimostrati in grado di spiegare e coinvolgere i giovani studenti nelle discipline a loro più vicine perché studiate all'università o per passioni personali, riportando la loro esperienza e riattivando le loro conoscenze. Ad esempio, la tutor madre lingua inglese ha potuto seguire meglio i ragazzi in difficoltà nello studio di tale lingua; il tutor laureato in Economia, ma con la passione per la filosofia, ha affiancato spesso un alunno con una buona competenza della lingua italiana nello studio della filosofia.

Il numero maggiore dei punti di forza riportati si riferisce al secondo insieme, si rifà dunque alla relazione, all'importanza di creare un buon clima fatto di fiducia, complicità e affetto. In questo senso gli studenti tutorati si sono sentiti sempre più "liberi", non giudicati ma supportati e in alcuni casi si è stabilito un vero rapporto di affetto, che andava oltre le ore di laboratorio.

Per quanto riguarda i punti di debolezza possiamo anche in questo caso individuare due filoni principali: la difficoltà di mettersi nei panni del "docente" e la difficoltà nel mettersi nei panni del discente.

Nel primo caso la difficoltà riscontrata è una difficoltà di natura pratica, oggettiva, legata in gran parte alle competenze della lingua italiana; nel secondo caso la difficoltà è più a livello relazionale, nasce dal desiderio di comprendere e si scontra, spesso, con la complessità che questo comporta. 
Per cercare di intaccare almeno in parte questa difficoltà, il gruppo si è confrontato sin da subito sulle competenze comunicative, in particolare sull'ascolto attivo, condividendo il fatto che "per comunicare è necessario ascoltare, senza ascolto non c'è comunicazione! È stato evidenziato che l'ascolto è un'abilità molto complessa che richiede formazione, impegno nell'applicazione, intenzionalità. Inoltre l'ascolto attivo presuppone un approccio alla comunicazione di tipo partecipativo, orientato alla valorizzazione dello scambio tra i soggetti coinvolti, attento alla componente emotiva, componente non sempre facile da gestire (Sclavi 2000).

Ascoltare attivamente infatti significa, essere empatici, mettersi «nei panni dell'altro», riconoscere e accettare il suo punto di vista, accogliendo e comprendendo le emozioni, i dubbi, le preoccupazioni che manifesta.

Nell'ambito del tutoraggio di alunni stranieri che talvolta non hanno la padronanza della lingua italiana, per esprimere al meglio ciò che sentono o pensavo o che possono manifestarsi in modo diverso da quello abituale, significa che il tutor deve essere disponibile a sentirsi «goffo», a riconoscere che fa fatica a comprendere ciò che l'altro ci sta dicendo.

In questo modo si sono stabiliti rapporti di riconoscimento, di rispetto e di apprendimento reciproco che sono la condizione per affrontare congiuntamente e creativamente il problema.

Ascolto passivo

Ascolto attivo

\begin{tabular}{|l|l|}
\hline Statico (unica prospettiva giusta) & $\begin{array}{l}\text { Dinamico (Una pluralità } \\
\text { di prospettive) }\end{array}$ \\
\hline Passivo (rispecchiare la realtà) & Attivo (costruzione della realtà) \\
\hline $\begin{array}{l}\text { In controllo (incidenti di percorso } \\
\text { e imbarazzi ritenuti negativi) }\end{array}$ & $\begin{array}{l}\text { Goffo (incidenti di percorso } \\
\text { e imbarazzi positivi) }\end{array}$ \\
\hline Oggettivo & $\begin{array}{l}\text { Né soggettivo, né oggettivo } \\
\text { (esploratore di mondi possibili) }\end{array}$ \\
\hline Neutralizzare le emozioni & Centralità delle emozioni \\
\hline
\end{tabular}

\section{Alcune immagini/metafore/rappresentazioni del tutor}

Di seguito, alcune immagini proposte raccontate dai tutor sul loro stile di tutoraggio, a volte protettivo, altre volte emancipatorio o incalzante. 


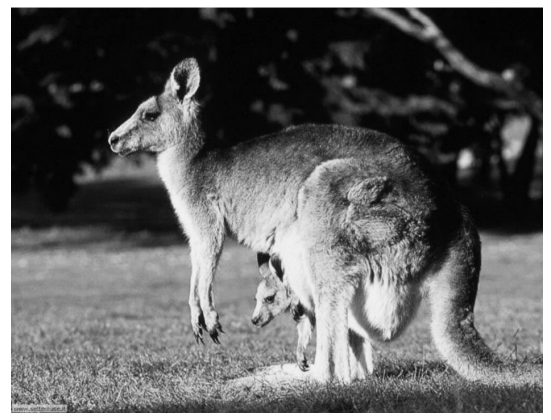

II tutor si prende cura del tutorato, lo stimola, lo accompagna nello studio e allo stesso tempo lo sorregge nei momenti delicati.

In questo caso, la relazione gioca un ruolo importante nell'apprendimento.

“Dopo un po' di imbarazzo iniziale il nostro rapporto è diventato molto forte, la ascolto volentieri quando mi parla di sé e delle sue difficoltà".

\section{Il fratello maggiore}

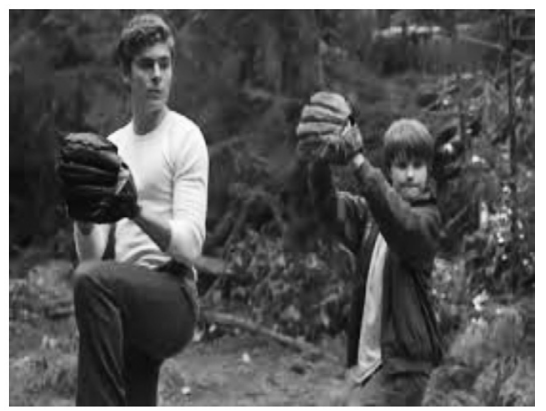

II tutor accompagna il tutorato con un buon equilibrio tra empatia e autorevolezza.

In questo caso, la relazione gioca un ruolo importante nell'apprendimento, ma non c'è confusione di ruoli.

"All'inizio sono stato piuttosto autorevole, ma con il passare del tempo e conoscendoci meglio è venuta un po' meno la parte autorevole in virtù di un rapporto paritario e di tipo "fratello maggiore".

\section{II martello}

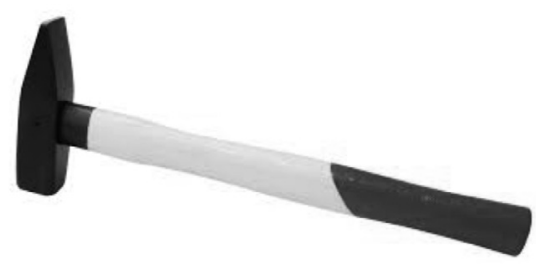

Il tutor segue con molta attenzione i progressi scolastici e organizza in modo meticoloso il suo intervento.

In questo caso, assume maggior peso il raggiungimento dell'obiettivo didattico.

"Ho cercato sempre di essere precisa e far capire in maniera chiara le cose! Sono certa di questo perché i ragazzi ritornavano dicendo che l'interrogazione o il compito scritto erano andati bene e questo è una soddisfazione non solo per loro ma anche per noi tutor in quanto siamo riusciti ad aiutarli!" 


\section{Riferimenti bibliografici}

Demetrio D., Favaro G. (2002), Didattica interculturale. Nuovi sguardi, competenze, percorsi, FrancoAngeli Milano.

Favaro G. (a cura di) (2013), Bussole sul cammino. Un progetto di accompagnamento scolastico per gli adolescenti stranieri, Fondazione "Peppino Vismara", Centro COME, Milano.

Gelli B.R., Mannarini T. (1999), Il mentoring. Uno strumento contro la dispersione scolastica, Carocci, Roma.

Luatti L., Tizzi G. (a cura di) (2014), La scuola e i "nuovi” italiani: accogliente, integrativa e inclusiva? Una ricerca esplorativa in provincia di Arezzo, Oxfam Italia, Arezzo.

Mariani L. (2006), La motivazione a scuola. Prospettive teoriche e interventi strategici, Carocci, Roma.

Polito M. (2000), Comunicazione in classe e apprendimento cooperativo, Erikson, Trento.

Sclavi M. (2003), Arte di ascoltare e mondi possibili. Come si esce dalle cornici di cui siamo parte, Carocci, Milano.

Sclavi M., Giornelli G. (2014), La scuola e l'arte di ascoltare, Feltrinelli, Milano. 


\section{6. "Almeno una stella" in Friuli Venezia Giulia: la scuola oltre la scuola}

di Flavia Virgilio*

\section{II progetto in Friuli Venezia Giulia}

I dati di contesto sulla presenza degli allievi stranieri ci dicono che oltre 200.000 alunni con cittadinanza straniera sono iscritti in una scuola - privata o paritaria - del Nord Est e 18.097 sono iscritti in Friuli Venezia Giulia. Rispetto all'anno precedente, gli alunni con cittadinanza non italiana (stranieri, apolidi e non classificati) sono cresciuti, a livello nazionale, del $6,4 \%$, incremento superiore a quello registrato nella ripartizione Nord Est ed in FVG dove l'aumento è stato rispettivamente pari al 5,6\% e al 5,2\% (Annuario Statistico Immigrazione FVG 2013). Nell'anno scolastico 2013/14 erano iscritti nelle scuole del Friuli Venezia Giulia 19.021 (9.978 dei quali nati in Italia), di cui 22,9\% nella scuola dell'infanzia, 34,8\% nella primaria, $19,7 \%$ nella secondaria di primo grado e $22,5 \%$ nella secondaria di secondo grado.

Le scuole aderenti al progetto rappresentano uno spaccato delle scuole regionali a più alta presenza di allievi stranieri e si distinguono per numerosità ed eterogeneità della presenza.

Complessivamente sono stati coinvolti nell'attività 7 istituti comprensivi ( 2 in provincia di Pordenone, 1 in provincia di Gorizia, 4 in provincia di Udine) e 5 istituti scolastici di secondo grado ( 1 in provincia di Udine e 4 in provincia di Pordenone). Le percentuali di allievi stranieri nei diversi istituti sono le seguenti:

- Istituto Comprensivo II di Udine 32\%

- Istituto Comprensivo III di Udine 37,14\%

- Istituto Comprensivo V di Udine $17,71 \%$

- Istituto Comprensivo di Latisana 20,95\%

- Istituto Comprensivo Pordenone Sud 31,7\%

- Istituto Comprensivo di Porcia (PN) 11,33\%

- Istituto Comprensivo Giacich di Monfalcone (GO) 24\%

- ISIS FLORA (PN) 20\%

- Liceo Grigoletti (PN) 7,14\%

La Regione Friuli Venezia Giulia ormai da più di dieci anni è impegnata a sostenere le istituzioni scolastiche con fondi del Piano regionale Immigrazione per favorire l'inclusione degli allievi stranieri. Il Piano ar-

\footnotetext{
* Gruppo di lavoro "Almeno una stella" Friuli Venezia Giulia - scuola capofila I.C. II di Udine.
} 
ticola diverse possibilità di finanziamento per le istituzioni scolastiche e territoriali, tra cui un progetto di formazione che negli ultimi dieci anni è sempre stato gestito da una rete regionale di scuole. Il gruppo di coordinamento di rete è costituito da sette docenti appartenenti ai ruoli della scuola di base e del secondo ciclo, coordinati da un dirigente scolastico.

Nell'arco degli anni la rete, che ha visto l'alternarsi di diversi istituti capofila, ha prodotto la versione regionale del Quaderno dell'Integrazione, iniziative di formazione, ricerca azione ed elaborazione di materiali e documentazione. Diverse attività, in particolare la produzione del Quaderno dell'Integrazione, la formazione e la selezione l'accompagnamento dei tutor universitari, sono state realizzate in collaborazione con l'Università di Udine.

Negli ultimi due anni, l'impegno della rete si è concentrato sulla sperimentazione di dispositivi di tutoraggio nella scuola secondaria di primo e secondo grado, secondo il modello del progetto "Almeno una Stella", accompagnando le scuole al consolidamento di buone pratiche di intervento nelle classi eterogenee e, in particolare, negli anni ponte tra la secondaria di primo e secondo grado. Nello specifico sono state realizzate attività di:

- coordinamento e rete; formazione e consolidamento del gruppo di coordinamento; allargamento e consolidamento della rete di scuole; intensificazione della collaborazione con I'Ufficio Scolastico Regionale; consolidamento della collaborazione con I'Università di Udine; ricerca di nuovi partner universitari; consolidamento della collaborazione con la Regione Friuli Venezia Giulia;

- formazione per i tutor universitari, i docenti delle scuole coinvolte, i dirigenti scolastici;

- produzione materiali (protocolli, materiali per il monitoraggio della situazione degli studenti, materiali di supporto per lo svolgimento dell'esame conclusivo del primo ciclo di istruzione);

- accompagnamento della continuità verticale tra primo e secondo ciclo di istruzione per gli studenti neoarrivati e orientamento al sistema scolastico per studenti e famiglie.

I tutor universitari coinvolti nei due anni sono stati 11, provenienti dal corso di laurea di Lingue e Letterature Straniere (7) e di Educazione Professionale (4). Tutti i tutor sono stati individuati e selezionati grazie ad una convenzione con l'Università di Udine. Per i tutor universitari e i docenti del gruppo di coordinamento è stata organizzata una formazione curata da Oxfam Arezzo sulle metodologie del tutoring e sulle specifiche modalità di intervento con allievi stranieri neoarrivati in collaborazione tra scuola ed extrascuola. 
Alle attività formative realizzate dal progetto nel corso degli ultimi due anni hanno partecipato 230 docenti, provenienti da istituti anche al di fuori della rete "Almeno una stella", una parte dei quali neoassunti in ruolo.

\section{I ragazzi accompagnati}

Nel corso dei due anni di sperimentazione, sono stati accompagnati 40 studenti (6 frequentanti la scuola secondaria di secondo grado e 34 l'anno conclusivo della scuola secondaria di primo grado).

Tutti gli allievi seguiti nel 2014 frequentavano la classe terza della scuola secondaria di primo grado.

Di seguito gli esiti alla fine di ogni anno.

24 studenti della secondaria di primo grado seguiti nell'a.s. 2013/14:

- 1 è rientrato al paese di origine senza concludere l'anno scolastico (4\%)

- 5 non sono stati ammessi all'esame conclusivo (20\%)

- II voto massimo all'esame finale è stato 10 (2 studenti); seguono 8 (studenti), 7 (5 studenti), 6 (8 studenti)

- 8 studenti hanno scelto un Istituto tecnico, 5 un Istituto professionale, 3 un Liceo, 2 un corso regionale di formazione professionale.

Su 16 studenti della secondaria di primo grado seguiti nell'a.s. 2014/15:

- tutti sono stati ammessi all'esame conclusivo

- 1 studentessa non ha superato l'esame $(6,2 \%)$

- il voto massimo all'esame finale è stato 8 (1 studente); seguono 7 (3 studenti), 6 (10 studenti), di un alunno non è stata comunicata la valutazione.

Su 6 studenti della secondaria di secondo grado seguiti nell'a.s. 2014/15:

- 2 non sono stati ammessi alla classe successiva, uno è sospeso.

I dati evidenziano una problematicità nel passaggio alla secondaria di secondo grado che riguarda: orientamento e tipologia della scuola scelta; modalità di accoglienza nella scuola secondaria di secondo grado; mancata continuità, nel passaggio tra i cicli, del percorso personalizzato specifico per gli allievi NAI. In alcuni casi, conflitti tra aspettative/scelte della famiglia, aspettative/scelte dei ragazzi, aspettative/offerta della scuola e dei docenti.

A queste criticità il progetto ha risposto rafforzando per quest'anno l'accompagnamento dei 16 allievi seguiti per la preparazione del esame conclusivo del primo ciclo da parte dei tutor.

L'accompagnamento è così strutturato: 
- presentazione dello studente al docente referente della scuola secondaria di secondo grado, consegna di eventuali materiali prodotti nell'ambito del progetto "Almeno una stella";

- presentazione delle attività del progetto "Almeno una stella", proposta alle nuove scuole di adesione e possibilità di prosecuzione del tutoraggio da parte degli studenti universitari nella prima parte dell'a.s. 2015/16;

- presentazione del tutoraggio come strumento per un possibile arricchimento degli interventi della scuola secondaria di secondo grado per gli allievi NAl e in generale per gli allievi stranieri;

- monitoraggio dei risultati scolastici a fine primo quadrimestre e a fine anno;

- elaborazione di un protocollo per l'accompagnamento degli allievi NAI nel passaggio alla secondaria di secondo grado;

- presentazione del protocollo in un incontro per docenti e Dirigenti nell'ottobre 2015/16.

\section{Patti educativi tra scuola ed extrascuola}

Oggi più che mai lavorare nell'ambito dell'educazione richiede ampie alleanze tra soggetti diversi. Se fino a vent'anni fa il nucleo del processo educativo erano la scuola e la famiglia, oggi inevitabilmente questo nucleo si articola e si allarga e determina la pluralizzazione non solo dell'offerta educativa ma anche degli attori dei processi educativi e dei luoghi dell'educazione (Chiosso et al., 2009, p. 56). La sperimentazione del progetto "Almeno una stella" in Friuli Venezia Giulia è stata una possibilità di riflessione proprio su questa pluralizzazione dei contesti educativi.

Come indicato nella premessa delle Indicazioni Nazionali, infatti, "l'orizzonte territoriale della scuola si allarga. Ogni specifico territorio possiede legami con le varie aree del mondo e con ciò stesso costituisce un microcosmo che su scala locale riproduce opportunità, interazioni, tensioni, convivenze globali" (MIUR, 2012, p. 7). Si ampliano e si sfrangiano anche le modalità, le strategie e i contesti dell'azione educativa tanto da far apparire a volte obsoleta la classica distinzione tra educazione formale, non formale e informale.

La scuola si apre alle famiglie e al territorio circostante, facendo perno sugli strumenti forniti dall'autonomia scolastica, che prima di essere un insieme di norme è un modo di concepire il rapporto delle scuole con le comunità di appartenenza, locali e nazionali. L'acquisizione dell'autonomia rappresenta un momento decisivo per le istituzioni scolastiche. Grazie a essa si è già avviato un processo di sempre maggiore responsabilizzazione condiviso dai docenti e dai dirigenti, che favorisce altresì la stretta connessione di ogni scuola con il suo territorio. In quanto comunità educante, la scuola genera una diffusa convivialità relazionale, intessuta di linguaggi affettivi ed emotivi, ed è anche in grado di promuovere la condivisione di quei valori che fanno sentire i membri della società come parte di una comunità vera e propria. La scuola affianca al compito dell'insegnare ad apprendere quello dell'insegnare a essere. (MIUR, 2012, p. 10) 
In questo contesto vanno perciò letti sia il senso che la funzione di un progetto come "Almeno una stella", che si propone come opportunità educativa in senso ampio, non solo di recupero e compensazione didattico disciplinare, costituendo di fatto un ponte tra scuole ed extrascuola, tra educazione formale e informale.

In Friuli Venezia Giulia il progetto, coordinato e promosso da una rete di scuole in collaborazione con l'Università di Udine, si colloca proprio nella direzione di fare della scuola un luogo in cui, attraverso la cooperazione con il territorio, si promuove la cittadinanza attiva e l'inclusione sociale, la convivialità relazionale e non solo il pur fondamentale successo scolastico. Anzi, possiamo dire che il progetto, attraverso la promozione del tutoraggio come strategia per il miglioramento delle opportunità di successo scolastico per gli allievi stranieri, promuove proprio nel territorio la consapevolezza di essere comunità educante.

Si può allora dire a buona ragione che "Almeno una stella" ha svolto un ruolo di ponte verso la scuola per gli alunni stranieri e le loro famiglie e, nel contempo, di ponte verso il territorio per i docenti e le scuole. Si può parlare di "una funzione sociale di recupero da situazioni di marginalità ed anzi di prevenzione di tali situazioni, ma soprattutto di una funzione pedagogica dimostrativa delle capacità di accoglienza che dovrebbe essere propria di tutta la scuola" (Colasanto, 2005, p. 11).

Il dispositivo del tutoraggio è stato vissuto da docenti, allievi stranieri e tutor universitari, non tanto e non solo come luogo di compensazione e di recupero di lacune e gap socioculturali, ma piuttosto come uno spazio intermedio di rimotivazione per ragazzi che per diverse ragioni si trovano in situazione di disagio scolastico e di riorganizzazione per le scuole, che si sono trovate a dialogare con soggetti e modalità di lavoro diversi da quelli strettamente curricolari. Si tratta, perciò, di un vero e proprio dispositivo non formale di sostegno e reintegrazione verso le istituzioni scolastiche, un ponte verso il successo formativo che passa attraverso la personalizzazione come riconoscimento dell'essere sé di ciascuno (Chiosso et al., 2009, p. 59).

È per questo che a buona ragione il progetto non è, insomma, solo un ospedale per i "malati" in difficoltà nella scuola (Scuola di Barbiana, 1967), ma un luogo di tessitura di relazioni, al centro di una costellazione di attività culturali (accoglienza, sostegno scolastico, networking sociale, uscite in città, ...) che vanno ben al di là del sostegno scolastico, promuovendo la costruzione di esperienze di equità, di scambio e di reciprocità. In questo senso "Almeno una stella" di sicuro contribuisce a colmare non solo le difficoltà di tipo scolastico, ma anche un profondo bisogno di cultura, segnalato da Save the Children nel $5^{\circ}$ Atlante dell'Infanzia 2014, che rileva come nell'ultimo anno l'84,9\% dei ragazzi (italiani e stranieri) non sia 
andato ad un concerto; il 73,7\% non abbia visitato un sito archeologico; il $72,1 \%$ non sia andato a teatro; il 60,8 non abbia visto una mostra e il 47,9 non abbia letto nemmeno un libro. Questi dati, tra l'altro dimostrano come la scuola fatichi a colmare il gap socioeconomico che è alla base delle diverse opportunità di successo scolastico per studenti provenienti da contesti familiari eterogenei (Dalla Zuanna, Farina, Strozza, 2009).

I giovani stranieri, anche quelli nati in Italia, - infatti - hanno risultati scolastici molto peggiori rispetto ai coetanei italiani; vengono bocciati e lasciano la scuola molto più di frequente rispetto ai figli di italiani; prendono voti più bassi, si iscrivono a scuole più professionalizzanti. Questo è un grosso problema per l'Italia, perché - come è accaduto in altri Paesi - se i giovani stranieri non avranno a disposizione risorse per raggiungere una posizione sociale migliore dei loro genitori, svilupperanno opposizione, rancore e antagonismo verso la società ospite e le sue regole. (Dalla Zuanna, Farina, Strozza 2009, p. 8)

L'esperienza di "Almeno una stella" è stata per la rete del Friuli Venezia Giulia una grande opportunità per ripensare l'agire educativo, anche della scuola, in una prospettiva territoriale e comunitaria, riflettendo sulle pedagogie del territorio (Sarracino, 2011), collocando le diverse esperienze realizzate in regione all'interno di una proposta complessiva. Infatti, se ci si focalizza sull'oggetto di un laboratorio o sulla specifica attività di sostegno scolastico, senza comprenderne la connessione con le progettualità educative complessive, si rischia di costruire contenitori slegati l'uno dall'altro non riconducibili ad un'unitarietà di percorsi. "Unitarietà che, viceversa, permette ad un ragazzo di percepire che al centro vi è lui, non il compito da portare a termine, e di comprendere l'importanza di quel momento, impegnandosi per valorizzarlo, cercando di viverlo in modo più pieno e se possibile piacevole, perché consapevole della sua utilità: un orizzonte di senso del proprio percorso di crescita" (De Bernardis, 2005, p. 40).

L'esperienza di "Almeno una stella" in Friuli Venezia Giulia ha cercato di percorrere questa strada. Mettendo al centro delle attività le relazioni, prima di tutto fra giovani studenti universitari e allievi della scuola media e superiore, ma anche fra tutor e docenti sia coordinatori che di classe. Questa "rete" di relazioni ha permesso di motivare o rimotivare tutti i partecipanti alla rete stessa realizzando così un lavoro d'équipe che ha creato l'opportunità di accompagnare gli studenti nella fase particolarmente delicata del passaggio di grado. Un progetto che ha dimostrato la possibilità e la necessità di azioni coordinate con il territorio e fra scuole anche di ordine diverso e che ci si auspica possa proseguire nel tempo. 


\section{Riferimenti bibliografici e sitografia}

Chiosso G., Ellerani P., Milani P., Moscato M.T., Rivoltella P.C. (2009), Luoghi e pratiche dell'educazione, Mondadori, Milano.

Colasanto M. (2005), Presentazione, in Educare altrove. L'opportunità educativa dei doposcuola, a cura di De Bernardis, A., FrancoAngeli, Milano, pp. 9-12.

Dalla Zuanna G., Farina P., Strozza S. (2009), Nuovi italiani. I giovani immigrati cambieranno il nostro Paese?, Il Mulino, Bologna.

De Bernardis A. (a cura di) (2005), Educare altrove. L'opportunità educativa dei doposcuola, FrancoAngeli, Milano, pp. 17-41.

MIUR (2012), Indicazioni nazionali per il curricolo della scuola dell'infanzia e del primo ciclo d'istruzione, Annali della Pubblica Istruzione, Le Monnier, Firenze.

Regione Friuli Venezia Giulia (2011), Interazioni: strumenti per l'integrazione. Il quaderno dell'integrazione delle scuole del Friuli Venezia Giulia, Regione Autonoma Friuli Venezia Giulia.

Save the Children (2014), $5^{\circ}$ Atlante dell'Infanzia (a rischio). in http://images. savethechildren.it/IT/f/img_pubblicazioni/img253_b.pdf.

Scuola di Barbiana (1967), Lettera e una professoressa, Libreria Editrice Fiorentina, Firenze.

Sarracino V. (2011), “Gli adulti, la partecipazione, lo sviluppo locale”, Pedagogia oggi, 1-2, pp. 93-104. 


\title{
7. Vademecum per un progetto di tutoraggio. Linee guida, obiettivi, strumenti
}

\author{
di Graziella Favaro
}

\section{Premessa}

Le indicazioni e i suggerimenti che vengono proposti di seguito per realizzare un progetto di tutoraggio sono l'esito di due progetti che il Centro COME ha realizzato come ente capofila e referente scientifico per conto della Fondazione "Peppino Vismara". Si tratta dei progetti denominati "Bussole" e "Almeno una stella", condotti fra il 2011 e il 2015 in sei diversi territori: Milano, Torino, Bologna, Arezzo, provincia di Trento, Friuli Venezia Giulia.

Le due azioni progettuali hanno avuto carattere sperimentale e sono state monitorate per poter acquisire dall'esperienza indicazioni e suggerimenti utili a proporre il dispositivo del tutoraggio in maniera più diffusa e in ambiti più ampi.

Due evidenze sono emerse in maniera chiara e documentata dai due progetti sperimentali:

- il tutoraggio si rivela positivo ed efficace per le ragazze e i ragazzi che vivono, o hanno vissuto in tempi recenti, la migrazione e il disorientamento rispetto alla nuova realtà, alla scuola, alla seconda lingua;

- l'azione del tutoraggio è altamente significativa per i tutor che assumono questo ruolo da "fratelli e sorelle maggiori" e che si trovano a vivere un'esperienza di cittadinanza attiva, di assunzione di un ruolo e di una responsabilità che hanno a che fare con i temi della cura e dell'empatia.

Perché l'azione di tutoraggio sia efficace e incisiva tuttavia, alcune condizioni devono essere previste fin dall'inizio e garantite strada facendo. Esse hanno a che fare con le caratteristiche dei tutor, il contesto in cui essi operano, il patto educativo chiaro e condiviso fra i vari soggetti in gioco.

Il Vademecum si propone di chiarire i requisiti e le condizioni per un progetto di tutoraggio efficace, distinguendo tra le fasi dell'azione e fornendo esempi e strumenti operativi e concreti. 


\section{Reperire i tutor}

I tutor sono giovani universitari che si impegnano per uno o due anni (a seconda della durata del progetto) ad accompagnare gli adolescenti stranieri di recente immigrazione a scuola e nella città. Nella fase iniziale, si stabiliscono i contatti con le università locali per uno o più incontri con gli studenti interessati e per una pubblicizzazione anche online (siti degli studenti, bacheche informative on line...).

Durante gli incontri in presenza o nella comunicazione a distanza, vanno chiariti:

- gli obiettivi del progetto;

- la sua durata;

- il ruolo che i tutor sono chiamati ad assumere;

- le condizioni della partecipazione al progetto.

Coloro che sono interessati a vivere l'esperienza del tutoraggio, compilano un semplice modulo di auto-presentazione.

In una fase successiva, avviene il colloquio con i giovani interessati a diventare tutor per individuare le loro disponibilità reali, le motivazioni e le aspettative. Nell'individuazione dei tutor, è importante seguire anche il criterio della loro eterogeneità: di genere, di tipologia di studi, di origine, di biografia linguistica, privilegiando coloro che hanno una storia diretta o famigliare di migrazione e sono bi o plurilingui.

In allegato, il programma di un incontro di presentazione del progetto (all. 1) in università e la scheda utilizzata per la raccolta dei dati sugli studenti che esprimono un interesse (all. 2).

\section{Formare i tutor}

La formazione dei tutor è un momento delicato che segna l'avvio del progetto e costruisce le basi perché si stabilisca un vero e proprio gruppo di lavoro.

La formazione dei tutor prevede, in genere, una durata di circa 20 ore diluita su incontri di 2 ore o su incontri di 3 ore ciascuno.

I temi che vengono presentati ai tutor hanno a che fare con:

- Ie finalità, gli obiettivi e il significato anche etico del progetto;

- le caratteristiche e la storia delle ragazze e dei ragazzi dell'immigrazione;

- le sfide le criticità legate all'inserimento in una nuova scuola e all'apprendimento attraverso una seconda lingua;

- il dispositivo del tutoraggio e il ruolo del tutor in una situazione formale e scolastica di accompagnamento degli adolescenti;

- le condizioni del tutoraggio e gli impegni del tutor. 
La metodologia utilizzata nel percorso formativo è attiva, partecipata. Cerca di agire sulle conoscenze e le informazioni, ma anche sulle rappresentazioni e sulle implicazioni relazionali.

In allegato, un esempio di percorso di formazione per i tutor (all. 3).

\section{Riconoscere l'esperienza di tutoraggio}

L'intervento dei tutor si svolge nell'ambito di un progetto formale e condiviso tra scuola, famiglia, associazionismo e università.

Esso è regolato anche da un accordo tra l'università e l'ente che lo realizza e prevede il riconoscimento del lavoro educativo e di accompagnamento dei tutor.

Il riconoscimento può essere dato in modo diverso:

- attraverso crediti per attività extracorsuali;

- con altre modalità stabilite dai docenti del corso di laurea frequentato dal tutor (valorizzazione dell'esperienza; relazione sull'intervento durante l'esame; riferimento all'esperienza nella tesi...).

In allegato: l'accordo stabilito con l'università Statale di Milano/corso di laurea in Mediazione linguistica e culturale (all. 4).

Di seguito, alcuni aspetti e attenzioni riferiti al profilo dei tutor.

\section{Profilo dei tutor}

- La vicinanza anagrafica

I tutor si collocano in un'età vicina, ma non pari, rispetto a quella dei ragazzi seguiti: rispetto a loro, non sono né compagni né adulti. La distanza anagrafica potrebbe essere compresa tra i 5 e i 10 anni.

- Un'appartenenza plurale

È un elemento estremamente positivo il fatto che il gruppo dei tutor sia multiculturale e internazionale e che comprenda, accanto a giovani italiani nativi, anche stranieri che hanno vissuto direttamente la migrazione, giovani stranieri di "seconda generazione" e italiani di origine straniera.

- La mescolanza di genere

Il gruppo dei tutor dovrebbe essere misto, per rendere possibile la ricchezza e la complessità relazionale che si genera dai comuni interes$\mathrm{si}$, dalle sensibilità e dalle vicende personali che sono più vicine e affini per genere. 
- La prossimità linguistica e il plurilinguismo

La presenza nel gruppo di giovani bi o plurilingui è un elemento immediato di valorizzazione delle lingue d'origine dei ragazzi stranieri; prefigura inoltre la possibilità concreta di un cammino di apprendimento linguistico in italiano positivo e composito ed è un importante segno, simbolico e reale, di riconoscimento e di inclusione.

- Linguaggi comuni

I tutor condividono con gli adolescenti l'uso più fluido e agile di canali e strumenti multimediali e questo si rivela positivo, sia nel lavoro didattico e dell'apprendimento, che nelle possibilità di continuare le relazioni a distanza.

- Il percorso formativo

I tutor frequentano corsi di studio universitari. In certi casi, possono anche essere studenti inseriti nelle classi finali della scuola secondaria di secondo grado Si osserva tuttavia che gli universitari hanno, in genere, una maggiore autorevolezza, una disponibilità più ampia e possono disporre di tempi più dilatati da dedicare all'esperienza.

- Il riconoscimento formale

L'impegno svolto come tutor nei progetti rivolti agli adolescenti immigrati deve essere riconosciuto dalle università e dalle scuole di provenienza in maniera formale, sia come crediti, sia come parte del percorso di tirocinio o di stage.

- Le esperienze educative e di volontariato

II lavoro di tutoraggio nei confronti di ragazzi più piccoli rappresenta un'esperienza importante e coinvolgente di assunzione di una responsabilità educativa diretta, di cura degli altri ed è un esercizio concreto di "cittadinanza attiva". Spesso chi si propone per questo ruolo ha avuto in precedenza esperienze di volontariato, di lavoro educativo informale o di servizio civile che vanno riconosciute e valorizzate.

- Dentro un progetto

I tutor accompagnano i ragazzi e le ragazze nell'ambito di un progetto educativo definito. Essi agiscono negli spazi e nei tempi scolastici o extrascolastici accanto a docenti ed educatori specialisti. L'impegno annuo è di 80-100 ore (circa 2 ore la settimana), compresi i momenti iniziali della formazione e del monitoraggio in itinere.

\section{Individuare gli alunni da accompagnare e stabilire ac- cordi con le scuole}

Le scuole coinvolte nel progetto (secondarie di primo e di secondo grado) indicano gli alunni che necessitano di un intervento di tutoraggio, individuandoli in maniera privilegiata fra gli studenti NAI (neoarrivati in Italia) o fra coloro che hanno una storia recente di immigrazione. 
Gli obiettivi "scolastici" dell'azione di tutoraggio hanno a che fare con le due criticità che maggiormente emergono nel percorso scolastico degli adolescenti e sono soprattutto i seguenti:

- sostenere le ragazze e i ragazzi stranieri nella preparazione all'esame di terza media;

- accompagnare le ragazze e i ragazzi stranieri durante il primo anno di inserimento nella scuola secondaria di secondo grado.

Le scuole coinvolte individuano un insegnante referente che segue il progetto, stabilisce e mantiene i contatti tra:

- i docenti delle classi;

- gli insegnanti specialisti;

- gli alunni coinvolti e le loro famiglie.

Insieme vengono definite le condizioni di base del tutoraggio:

- lo spazio in cui si terrà: scolastico o extrascolastico;

- i tempi: la durata (in genere, tra le 50 e le 80 ore) e la cadenza (un incontro settimanale di 3 ore o due incontri d due ore);

- l'équipe di progetto, formata dall'insegnante specialista e dai tutor coinvolti.

Il docente referente facilita anche la raccolta dei dati che riguardano l'alunno accompagnato, riferiti sia al percorso scolastico pregresso che agli esiti dell'intervento di tutoraggio.

In allegato: la scheda/tipo sullo studente e sul percorso scolastico (all. 5).

\section{Stabilire un "patto di tutoraggio" fra i soggetti coinvolti}

Dopo la fase di formazione dei tutor, e dopo aver stabilito gli accordi di base con le scuole, il progetto di tutoraggio può prendere avvio.

I tutor vengono assegnati alle diverse sedi e scuole nelle quali viene realizzato il progetto e vengono "abbinati" ai ragazzi che seguiranno. La scelta dell'abbinamento (in genere, a ogni tutor vengono assegnati due o tre ragazzi) è delicata e flessibile, al tempo stesso. Si tratta di tener conto di fattori quali: genere, provenienza, lingua d'origine del tutor. L'abbinamento è utile a stabilire un legame reciproco fra tutor e tutorato e l'assunzione di una responsabilità particolare da parte del tutor verso i ragazzi che gli sono stati assegnati. Nei momenti dell'affiancamento, i tutor possono naturalmente interagire in maniera flessibile con tutti gli alunni sulla base del compito da svolgere, delle competenze di ciascuno, del corso di studi frequentato. 
È importante stabilire un patto educativo chiaro e responsabile con l'alunno e con la famiglia, nel quale siano indicati le condizioni e gli obiettivi dell'intervento, i nomi dei docenti e dei tutor coinvolti.

I ragazzi accompagnati e i loro genitori si impegnano a garantire la frequenza, a essere puntuali, a rispettare i tempi e i compiti assegnati.

In allegato: il "patto di tutoraggio" steso e utilizzato nella provincia di Trento (all. 6).

Da parte loro, i tutor cercano di conoscere e far emergere i talenti e le risorse dei ragazzi che seguono, ascoltandoli e facendo in modo che possano mobilitare le capacità, i saperi e le emozioni positive per far fronte alla nuova sfida.

In questo senso, i tutor funzionano anche come "tutori di resilienza" (all. 7).

\section{Definire e gestire le quattro fasi del progetto}

I progetti sperimentali di tutoraggio fin qui realizzati consentono di articolare l'intervento in quattro fasi, ognuna delle quali richiede una gestione attenta delle dinamiche relazionali, dei ruoli di ciascuno, degli aspetti organizzativi.

Le fasi sono le seguenti:

- le mosse di avvio e l'assunzione del ruolo;

- l'accompagnamento educativo e la centralità del compito scolastico;

- l'approfondimento della relazione educativa e della conoscenza reciproca;

- la chiusura dell'intervento e il commiato.

\begin{tabular}{|l|l|l|}
\hline Prima fase & $\begin{array}{l}\text { Formazione dei } \\
\text { tutor e avvio } \\
\text { dell'esperienza }\end{array}$ & $\begin{array}{l}\text { - I tutor seguono un percorso formativo iniziale di } \\
\text { 4/5 incontri (circa 20 ore) } \\
\text { - Si provvede a formare i sottogruppi, eterogenei } \\
\text { per genere, età, nazionalità e lingua e a fare l'abbi- } \\
\text { namento con le diverse scuole, con i docenti e con } \\
\text { gli alunnitutorati. } \\
\text { - Nella fase di avvio, i tutor si presentano ai ragaz- } \\
\text { zi; chiariscono il loro ruolo e cercano di stabilire la } \\
\text { prima conoscenza dei ragazzi che seguiranno. }\end{array}$ \\
\hline Seconda fase & $\begin{array}{l}\text { Centralità } \\
\text { del compito } \\
\text { scolastico e del } \\
\text { patto formativo }\end{array}$ & $\begin{array}{l}\text { - Nella seconda fase, i tutor agiscono soprattutto } \\
\text { come facilitatori di apprendimento (tutor scolastico). } \\
\text { - La centralità è occupata dal compito da superare } \\
\text { e dall'obiettivo da raggiungere (ad esempio, prepa- } \\
\text { rarsi a superare l'esame di terza media). } \\
\text { - Fra i tutor e i ragazzi seguiti si stabilisce un patto } \\
\text { formativo esplicito che riguarda il compito. } \\
\text { - In questa fase, i tutor aiutano i ragazzi a studiare, } \\
\text { ricercare, affinare capacità e competenze. } \\
\text { - E cercando di aiutarli anche a organizzarsi e a } \\
\text { trovare un proprio metodo di studio, rifacendosi } \\
\text { anche alla propria esperienza di alunno. }\end{array}$ \\
\hline
\end{tabular}




\begin{tabular}{|c|c|c|}
\hline Terza fase & $\begin{array}{l}\text { L'approfondirsi } \\
\text { della relazione } \\
\text { e dello scambio }\end{array}$ & $\begin{array}{l}\text { - In una fase successiva, via via che la conoscenza } \\
\text { si approfondisce, i ragazzi stabiliscono con il tutor } \\
\text { una relazione di apertura e fiducia. } \\
\text { - Pongono domande e cercano risposte che hanno } \\
\text { a che fare con la vita di ogni giorno, le amicizie e gli } \\
\text { affetti, i progetti per il futuro... } \\
\text { - Esprimono i loro disorientamenti e timori, cerca- } \\
\text { no conferme e consigli. Il ruolo del tutor si fa più } \\
\text { delicato e complesso, ma può rivelarsi efficace per } \\
\text { rassicurare, rimotivare, ridare fiducia in se stessi. } \\
\text { - In questo caso, oltre ad essere un "tutor scolasti- } \\
\text { co", il giovane, italiano o straniero, può rappresen- } \\
\text { tare per gli adolescenti un esempio e un "modello" } \\
\text { di identificazione positiva (tutor di relazione e di } \\
\text { resilienza). }\end{array}$ \\
\hline Quarta fase & $\begin{array}{l}\text { La chiusura } \\
\text { e il commiato } \\
\text { (non definitivo) }\end{array}$ & $\begin{array}{l}\text { - Al termine dell'intervento di tutoraggio, i tutor e } \\
\text { gli adolescenti fanno il "bilancio" del loro incontro: i } \\
\text { ragazzi sono più autonomi? Che cosa hanno impa- } \\
\text { rato? Credono un po' di più in se stessi? } \\
\text { - E i tutor, che cosa hanno imparato da questa } \\
\text { esperienza? } \\
\text { - Oltre che il momento per raccontare e ripercor- } \\
\text { rere il cammino fatto insieme dai due punti di vista, } \\
\text { il commiato è anche l'occasione per darsi nuovi } \\
\text { appuntamenti, mantenendo dei fili e la possibilità } \\
\text { di ritrovarsi. }\end{array}$ \\
\hline
\end{tabular}

\section{Curare le relazioni, in presenza e a distanza}

Dopo la prima fase di presa di distanza e attesa reciproca, piano piano la relazione fra i giovani tutor e gli adolescenti accompagnanti si fa più salda e profonda. Le ragazze e i ragazzi stranieri si sentono seguiti, incoraggiati, orgogliosi di poter contare su figure di prossimità "prestigiose" che ce l'hanno fatta e che diventano modelli di identificazione positivi.

La relazione tra tutor e tutorati si costruisce nelle situazioni di apprendimento attorno al compito scolastico da svolgere e alla sfida da superare, ma si rinsalda anche e soprattutto nei momenti informali, prima e dopo lo studio, quando c'è il tempo per uno scambio più amicale e ludico.

Un'altra occasione importante per approfondire la relazione con le ragazze e i ragazzi seguiti è data dalle iniziative di accompagnamento da parte dei tutor alla scoperta della città e dei luoghi comuni e di aggregazione. Spesso questi sono ignorati e non frequentati dagli adolescenti stranieri di recente immigrazione. Le visite ai luoghi pubblici e importanti della città e del quartiere, l'accompagnamento a praticare attività sportive, l'orientamento a usare le risorse per tutti: sono anch'esse forme di tutoraggio e rappresentano momenti topici di apertura e di reciproco scambio. 
I giovani tutor e i ragazzi seguiti spesso continuano il dialogo anche a distanza grazie ai social network e alle modalità comunicative online. In questo modo i consigli sulla lezione da preparare, sui compiti da fare per il giorno dopo, o anche solo su un problema quotidiano e pratico, passano attraverso lo scambio a distanza. Tutor e tutorati condividono quasi sempre i linguaggi multimediali in maniera sicura e fluida.

Di seguito: un esempio di questionario sulla conoscenza della città e dei luoghi di aggregazione sottoposto agli studenti torinesi (all. 8).

\section{Monitorare e supportare le azioni di tutoraggio}

Durante l'esperienza di tutoraggio, i tutor possono contare su momenti di incontro dei gruppo e di supervisione (almeno tre) per mettere in comune e scambiare le riflessioni, i dubbi, le criticità che possono emergere durante il cammino.

I tutor sono inoltre invitati a tenere un "diario di bordo" nel quale raccontano l'esperienza e propongono le loro considerazioni. Il diario di bordo è articolato nelle parti seguenti:

Prima dell'esperienza

- auto-presentazione

- rappresentazione delle ragazze e dei ragazzi immigrati

- rappresentazione del tutoraggio e delle funzioni del tutor

Durante l'esperienza

- auto-osservazione e descrizione di 5 giornate/tipo: situazione; bisogni e richieste dei ragazzi; ruolo e funzioni del tutor

Dopo l'esperienza

- la storia dei ragazzi seguiti

- considerazioni sul tutoraggio

- punti di forza e criticità

- proposte

In allegato: diario di bordo del tutor (all. 9).

\section{Gestire la fase di chiusura del progetto}

La fase di chiusura del progetto è delicata e complessa per varie ragioni. $\mathrm{Si}$ interrompe l'appuntamento settimanale, ormai diventato consuetudine, fra i tutor e i tutorati; si traccia un bilancio dell'esperienza; si registrano gli esiti del progetto e si valuta la sua efficacia. 
Per quanto riguarda l'aspetto relazionale, i tutor hanno tessuto nel periodo di accompagnamento educativo dei legami importanti e dei fili di attenzione nei confronti degli adolescenti che hanno seguito. Legami che ora si interrompono, ma che si potranno in parte mantenere attraverso le modalità di comunicazione a distanza.

In questa fase, è importante raccogliere il punto di vista delle ragazze e dei ragazzi che sono stati seguiti per registrare il loro punto di vista, la valutazione dei protagonisti, definire ciò che ha funzionato oppure no, per poter rivedere in seguito gli aspetti problematici.

Per quanto riguarda gli esiti, i tutor collaborano alla raccolta dei dati finali e degli esiti scolastici insieme agli insegnanti. Possono contattare i ragazzi per verificare quali sono le loro scelte scolastiche, segnalare eventuali casi o rischi di dispersione scolastica. Possono inoltre verificare che all'inizio del nuovo anno scolastico, gli alunni pre-iscritti siano effettivamente inseriti nella scuola che avevano scelto.

In allegato: un esempio di questionario sulla valutazione dell'esperienza di tutoraggio da parte dei ragazzi accompagnati (all. 10).

\section{Valutare, documentare, condividere}

Come tutti i progetti, anche quello sul tutoraggio deve essere documentato e verificato per capirne la validità e misurarne l'efficacia.

In quale modo si può fare?

Possiamo usare strumenti e indicatori diversi di tipo quali-quantitativo.

Tra gli indicatori:

- Ie modalità della frequenza ai corsi proposti;

- gli esiti scolastici degli alunni seguiti;

- il livello linguistico in entrata e in uscita;

- le scelte scolastiche dopo la terza media;

- la conoscenza della città e la frequenza di luoghi di aggregazione prima e dopo l'intervento di tutoraggio.

Tra gli strumenti:

- la raccolta puntuale e accurata degli esiti scolastici de ragazzi seguiti e del loro percorso scolastico, pregresso e in previsione;

- il diario di bordo dei tutor;

- il questionario di valutazione proposto ai ragazzi accompagnati;

- dei focus group con gli insegnanti coinvolti nelle azioni, siano essi docenti specialisti dell'i insegnamento dell'italiano L2 e di percorsi di integrazione, siano essi docenti di classe. 


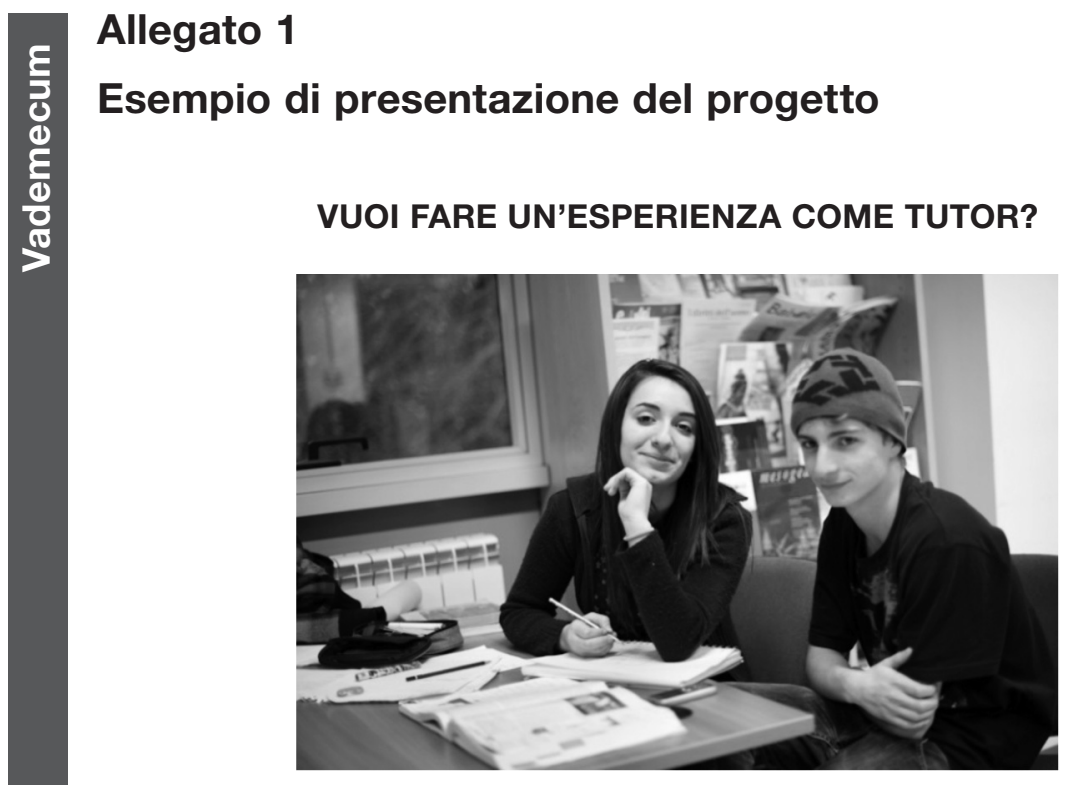

\section{II progetto "Almeno una stella"}

II progetto biennale "Almeno una stella" si propone di sostenere e accompagnare il cammino scolastico degli adolescenti stranieri, soprattutto di recente immigrazione, durante due anni cruciali: in terza media e nel primo anno della scuola superiore. II progetto è promosso e gestito dal Centro COME della cooperativa sociale "Farsi Prossimo" e si realizza, con la partecipazione di altri partner, in diverse città italiane.

Nell'ambito del progetto, e in collaborazione con le università milanesi, cerchiamo giovani universitari italiani e stranieri, da inserire nelle attività, che assumano il ruolo di tutor e di "stelle", e accompagnino il cammino di ragazze e ragazzi di età inferiore, nella scuola e nella città.

Che cosa chiediamo: a ciascun tutor chiediamo un impegno di 120 ore complessive, compresi alcuni momenti di formazione, a partire da gennaio 2014 fino a giugno 2015. A fronte di tale impegno, i tutor riceveranno un gettone come riconoscimento delle spese.

Che cosa offriamo: coinvolgeremo i tutor in una formazione specifica a carattere didattico-educativo, in interventi di sostegno scolastico (laboratori in preparazione all'esame di terza media e di aiuto allo studio nella scuola superiore) presso le scuole selezionate, in attività di accompagnamento e conoscenza del territorio.

I tutor saranno sempre affiancati dagli educatori/insegnanti che operano nel progetto.

Riconoscimento: le università riconoscono questa attività con modalità definite caso per caso.

Per ulteriori informazioni, telefonare al Centro COME 02-67.10.07.92 oppure inviare una mail a info@centrocome.it 
Allegato 2

Scheda di raccolta della disponibilità dei tutor

\author{
ALMENO UNA STELLA \\ I giovani tutor accompagnano gli adolescenti stranieri a scuola \\ e nella città
}

SCHEDA ANAGRAFICA

$\mathrm{M} \square \mathrm{F} \square$

1. DATI

\begin{tabular}{|l|}
\hline Cognome nome: \\
\hline Età: \\
\hline Nazionalità: \\
\hline Lingue conosciute: \\
\hline $\begin{array}{l}\text { Ente di appartenenza (se non proveniente da università e scuole del pro- } \\
\text { getto): }\end{array}$ \\
\hline Indirizzo: \\
\hline Telefono: \\
\hline Mail: \\
\hline
\end{tabular}

\title{
2. SCOLARITÀ
}

Studente universitario (nome Università)

Facoltà

Docente referente per il progetto

\section{EVENTUALI ESPERIENZE PREGRESSE DI VOLONTARIATO}

Ente

Attività di volontariato presso

dal

al

Acquisite le informazioni fornite dal titolare del trattamento ai sensi dell'articolo 13 del D.Lgs. 196/2003 (Consenso a trattare i dati comuni per le attività indispensabili al proseguimento del rapporto; consenso al trattamento dei dati sensibili e/o giudiziari; consenso al trattamento dati audio video) presto il mio libero consenso al trattamento e alla comunicazione dei miei dati personali, sensibili e audio video come risultante dalla suddetta informativa, limitatamente comunque al rispetto di ogni altra condizione imposta per legge.

Data 


\section{Allegato 3}

\section{Esempio di percorso di formazione/tipo}

\section{Note sul percorso formativo}

- I destinatari

Gli studenti italiani e stranieri che daranno la loro disponibilità ad assumere il ruolo di tutor o mentore nei confronti dei ragazzi stranieri hanno le caratteristiche seguenti: giovani universitari - di vent'anni e oltre - che frequentano i corsi di laurea di Scienze della formazione (Bicocca e Cattolica) e di Mediazione linguistico-culturale (Statale)

- Temi e contenuti

II percorso formativo rivolto ai tutor si compone di 4 incontri:

$-1^{\circ}$ incontro

Breve presentazione del progetto

II contesto: le scuole milanesi diventate multiculturali. Dati e descrizione dei contesti/delle zone

$-2^{\circ}$ incontro

Gli adolescenti stranieri: caratteristiche, bisogni, percorsi scolastici e criticità. Alcuni casi e storie di migrazione

$-3^{\circ}$ incontro

Chi è il tutor. Che cosa fa

Figure di tutor nella mia vita (racconti autobiografici dei partecipanti)

Diario di bordo: presentazione

$-4^{\circ}$ incontro

Aspetti organizzativi e suddivisione dei tutor per zona e attività Incontro con gli insegnanti referenti delle scuole con i quali lavoreranno 


\title{
Allegato 4
}

\section{Accordo stabilito con l'università Statale di Milano/corso di laurea in Mediazione linguistica e culturale}

\author{
PROTOCOLLO N. 00026/CD Med-Lim
}

VERBALE DEL COLLFGIO DIDATTICO

In Collegio Didartico Mod-Lin ai trumito martadi 3 dicembre 2013 allo ore 10.00 in Aula Magna (Pisma Indro Momtanolli 1, pismterrano) per la trattariono dal wgasate:

\section{ORDINE DHL GIORNO}

1. Approvazione verbele della seduta precedente;

2. Consmicarioni;

3. Critari ANVUR-AVA - rarionalizurions dell'offarta didattica par il trianmio 2014-2017;

4. Colleborarioni ax art. 47 por l'unno accadamico 2013-2014;

5. A.A. 2013-2014: Cultori della mestaria:

6. Provvodimonti riguardanti la didattica;

7. Comvarione tra 1'Universita di Valledolid o 1'Universith degli Studi di Milaso per un programema di doppio titolo:

8. Pratiche stadenti;

9. Pratiche Ensmun;

10. Vario od oventuali.

Soso prosenti (P), assanti givstificati (AG), altri nsonti (A):

(..)

6. Provrodimenti riguardanti la didattica

Il Prosidemte chiede al Collegio di delibsrare in merito ai wegenti provvedimeati:

- (...)

- Viene proposta l'aderiene al Progetto Almeno una stella, realizzato dalla Cooparativa Farsi Prossimo ONLUS Ses. o dal Canto COMF, con i quali gis in fobbraio avovamo deliberato la parteciparione a un progatto di tutoraggio, mall'ambito dai progotti FEI, svolto con il Consane di Milano o l'Unione Europoz. Talo imiriativa viane roalinnata ora anche con organimzrioni o commini di altre citts (Arozzo, Bologna, Torino). I Progetto Abmeno una stella prerode por gli studenti univorvitari un'attivita di tutoraggio, volta a sostenare accompagsare il cammino vcolastico degli adolescenti stramieri di rocente immigrazione duranto dei anmi craciali: in torza nodia o nol primo amno della vcoola vaperiore (i risultati del progetto sono contanuti nal Rapporto finsle). Uno degli aspatti innovativi o sparimantali dal progacto ha riguendato la prewens anlo attivita di vostepoo o di ainto allo studio di giovemi italiani - straniari con il ruolo di tutor. Questa figur di prossimita si a rivalata estramsmento poritiva ed officace o ha permono un coimvolgimento maggione e m riconoscimento pî immediato degli alami

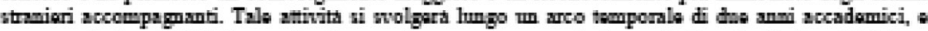
conateri di circa 120-150 oro, che daranno lnogo anche a un rimborno forfattario di circa 600 Euro, oltro che all'scquivirione di 6 cfa.

In Presidente chiode al Collogio di delibarane por gli studenti di MED o LDV la posvibilita di svolgare attivith di tatoraggio nell'ambito del progatto Almeno una stella, attivith che dara luogo all'acquisizione di 6 cfil. II Collagio approva all'unamimith.

(..)

Alle cre 11.30, osamrito il dibattito sui divorni punti all'ordine del gionso, la saduta o tolta.

II Providante

Prof. Giovnmi Turchath
II Segratario verbalizzamte

Dott:asa Roberta Gumuccio 


\section{Allegato 5}

\section{Scheda/tipo sullo studente e sul percorso scolastico}

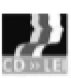

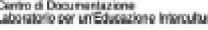

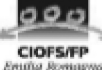
ting a.

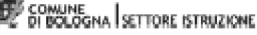
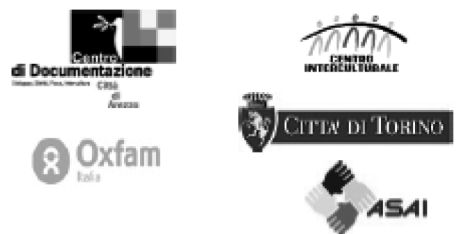

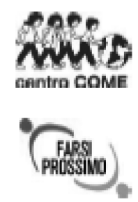

ALMENO UNA STELLA

I giovani tutor accompagnano gli adolescenti stranieri

SCHEDA STUDENTE

1. DATI ALUNNO

$\mathbf{M} \square \mathbf{F} \square$

Cognome e nome:

Paese di provenienza:

Luogo e data di nascita:

Luogo e data di arrivo in Italia:

2. SCOLARITÀ NEL PAESE D'ORIGINE

$N^{\circ}$ amni di scuola primaria:

$\mathrm{N}^{\circ}$ anni di scuola secondaria di $1^{\circ}$ grado, frequentati fino alla fine dell'anno scolastico:

Giudizio sintetico dell'ultimo anno scolastico frequentato:

3. SCOLARITA IN ITALIA

Data di iscrizione per la prima volta nel sistema scolastico italiano:

Scuola:

Classe e sezione:

Ha frequentato la scuola secondaria di $1^{\circ}$ grado per $\mathrm{N}^{\circ}$ anni:

\begin{tabular}{l|l}
\hline L'alumno è in pari rispetto alla sua età anagrafica? & Se no, indicare $\mathrm{N}^{\circ}$ anni di ritardo:
\end{tabular}

4. ESITI PROVE ESAME DI STATO CONCLUSIVO DEL PRIMO CICLO DI ISTRUZIONE (da compilare dopo l'esame)

Licenziato con il giudizio sintetico di: $/ 10$

\section{ISCRIZIONE ALLA SCUOLA SUPERIORE}

Iscrizione alla scuola secondaria di secondo grado:

Motivazione alla scelta della scuola secondaria di secondo grado: 


\section{LINGUA/E D'ORIGINE DELL'ALUNNO}

Qual è/Quali sono?

\section{CONOSCENZA DELL'ITALIANO COME SECONDA LINGUA}

Percorso di apprendimento

Ha seguito un corso di insegnamento della L2?

\begin{tabular}{|l|l}
\hline Per quante ore settimanali? & Per quanto tempo? \\
\hline
\end{tabular}

Organizzato da:

\section{Abilità}

\begin{tabular}{|l|c|c|c|c|c|c|}
\hline Ampiezza del lessico & Al & A2 & B1 & B2 & C1 & C2 \\
\hline Comprensione orale generale & & & & & & \\
\hline Interazione orale generale & & & & & & \\
\hline Comprensione generale di un testo scritto & & & & & & \\
\hline Produzione scritta generale & & & & & & \\
\hline Padronanza ortoggafica & & & & & & \\
\hline Correttezza grammaticale & & & & & & \\
\hline
\end{tabular}

Esito finale all'esame di $3^{\circ}$ media:

Esito finale della classe $1^{\circ}$ della scuola secondaria di secondo grado:

Esito finale della classe $2^{\circ}$ della scuola secondaria di secondo grado:

Note: (riorientamento, rientro nel paese d'origine, abbandono....)

Data di compilazione

A cura di:

Firmato: 


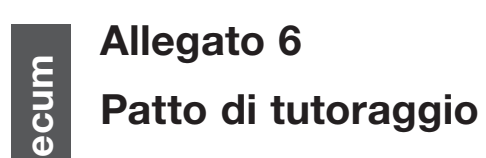

WOW, che bello, ho la possibilità di partecipare al progetto

ALMENO UNA STELLA!!

Mi incontrerò con la mia tutor

il martedì, dalle 14.00 alle 16.00

in biblioteca a ..............

per

parlare, giocare e lavorare col computer, studiare e fare i compiti, scoprire cosa c'è di interessante nel posto in cui vivo, andare a visitare Trento, il Muse, ecc.

So che è una cosa molto importante per me e accetto di partecipare con tutto il mio impegno!

Firma:

Firma genitori:

Pergine, 27 ottobre 2014 


\title{
Allegato 7
}

\section{Scheda per l'osservazione delle caratteristiche di resi- lienza dei ragazzi}

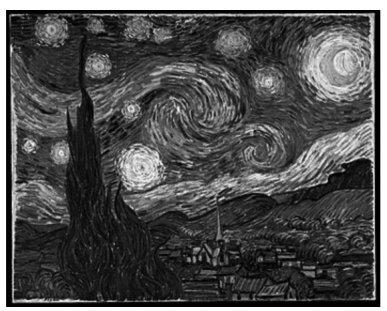

\author{
ALMENO UNA STELLA \\ I giovani tutor \\ accompagnano gli adolescenti stranieri \\ a scuola e nel territorio
}

a. Da 1 a 10 come stai? Cosa vorresti cambiare della situazione che stai vivendo?

b. Quali capacità e risorse pensi di avere? Quali sono gli aspetti del tuo carattere, della tua esperienza di vita che riconosci come positivi?

c. Cosa ti piace fare? In cosa sei creativa/o?

Quali sono le tue passioni?

Cosa ti piacerebbe fare e non ci sei ancora riuscita/o?

d. Per fare le cose che ti piacciono, come ti organizzi? Chiedi aiuto? Chiedi informazioni?

e. Di fronte ad una situazione di difficoltà, come reagisci? Cosa fai di solito per stare meglio nei momenti difficili?

f. Nelle relazioni (a scuola e fuori dalla scuola), quali sono le tue qualità? E quali sono le tue difficoltà?

Con chi ti trovi più a tuo agio? Con chi ti trovi a disagio?

g. Hai vissuto o stai vivendo situazioni di conflitto? Con chi? Cosa fai o cosa hai fatto per risolverle?

h. Quando ridi? Sai ridere anche di te stessa/o? Fammi un esempio.

Nelle situazioni di difficoltà, riesci a sdrammatizzare?

i. A chi racconti le tue esperienze? A chi racconti la tua storia?

I. Sai che le persone hanno diversi tipi di intelligenza:

- logico-matematica

- linguistica-verbale

- spaziale

- musicale

- cinestetica (del corpo)

- relazionale

- ..........

In quali di queste intelligenze ti riconosci di più e ti sembra di essere più dotata/o? 


\section{Allegato 8}

\section{Questionario sulla conoscenza della città}

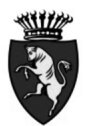

CiTTA' DI TORINO

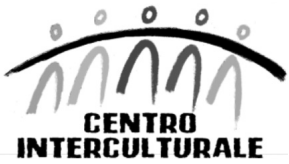

DIREZIONE CULTURA, EDUCAZIONE E GIOVENTU'

PROGETTO ALMENO UNA STELLA - LA MIA CITTÀ

Nome Cognome

Dove vivi?

Che luoghi conosci del tuo quartiere?

Quali sono i luoghi che conosci di Torino? E qual è il tuo luogo preferito?

Quando esci di solito dove vai?

$>$ Con che mezzi ti sposti in città?

$>$ I tuoi genitori ti lasciano uscire liberamente?

$>$ Ci sono dei luoghi importanti per te nel paese d'origine dei tuoi genitori?

Dove ti senti a casa? 


\section{Allegato 9}

\section{Diario di bordo del tutor}

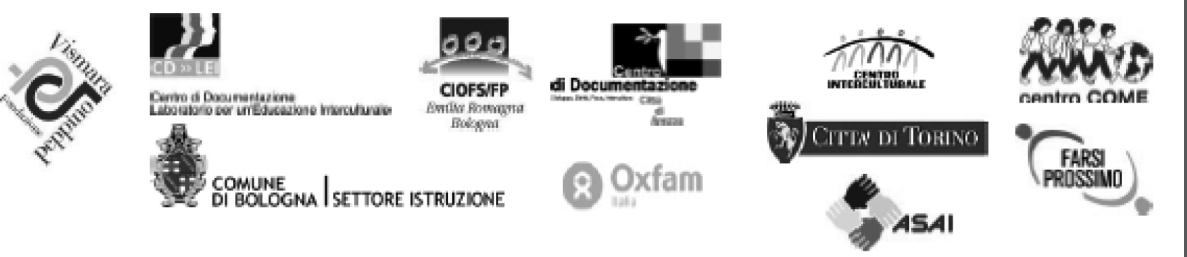

ALMENO UNA STELLA

I giovani tutor accompagnano gli adolescenti stranieri a scuola e nella città

DIARIO DI BORDO DI

\section{MI PRESENTO}

1. Auto-presentazione (la mia storia; il mio percorso scolastico passato, la formazione attuale, i miei progetti per il futuro ...) 
2. Che cosa penso dei ragazzi e delle ragazze immigrati (la loro storia, le loro difficoltà...)

3. Come immagino il mio ruolo di tutor (prima dell'esperienza) 


\section{DIARIO DELL'ESPERIENZA}

Prima osservazione

Data:.

4.1. Descrivo la situazione (spazio, tempi, presenze, relazioni nel gruppo, "clima"...)

\subsection{Le richieste, $i$ bisogni, gli atteggiamenti dei ragazzi}

4.3. Il mio intervento (azioni, compiti, atteggiamenti, materiali usati, esiti...) 
Seconda osservazione

Data:

5.1. Descrivo la situazione

5.2. Le richieste, $i$ bisogni, gli atteggiamenti dei ragazzi

5.3. Il mio intervento 
Terza osservazione

Data:

6.1. Descrivo la situazione

6.2. Le richieste, $i$ bisogni, gli atteggiamenti dei ragazzi

6.3. Il mio intervento 


\subsection{Descrivo la situazione}

7.2. Le richieste, i bisogni, gli atteggiamenti dei ragazzi

\subsection{Il mio intervento}


Quinta osservazione

Data:

8.1. Descrivo la situazione

8.2. Le richieste, $i$ bisogni, gli atteggiamenti dei ragazzi

\subsection{Il mio intervento}


III. I RITRATTI: LE RAGAZZE E I RAGAZZI CHE HO ACCOMPAGNATO

9. Racconto la storia personale, migratoria, scolastica delle ragazze/ dei ragazzi che ho accompagnato 


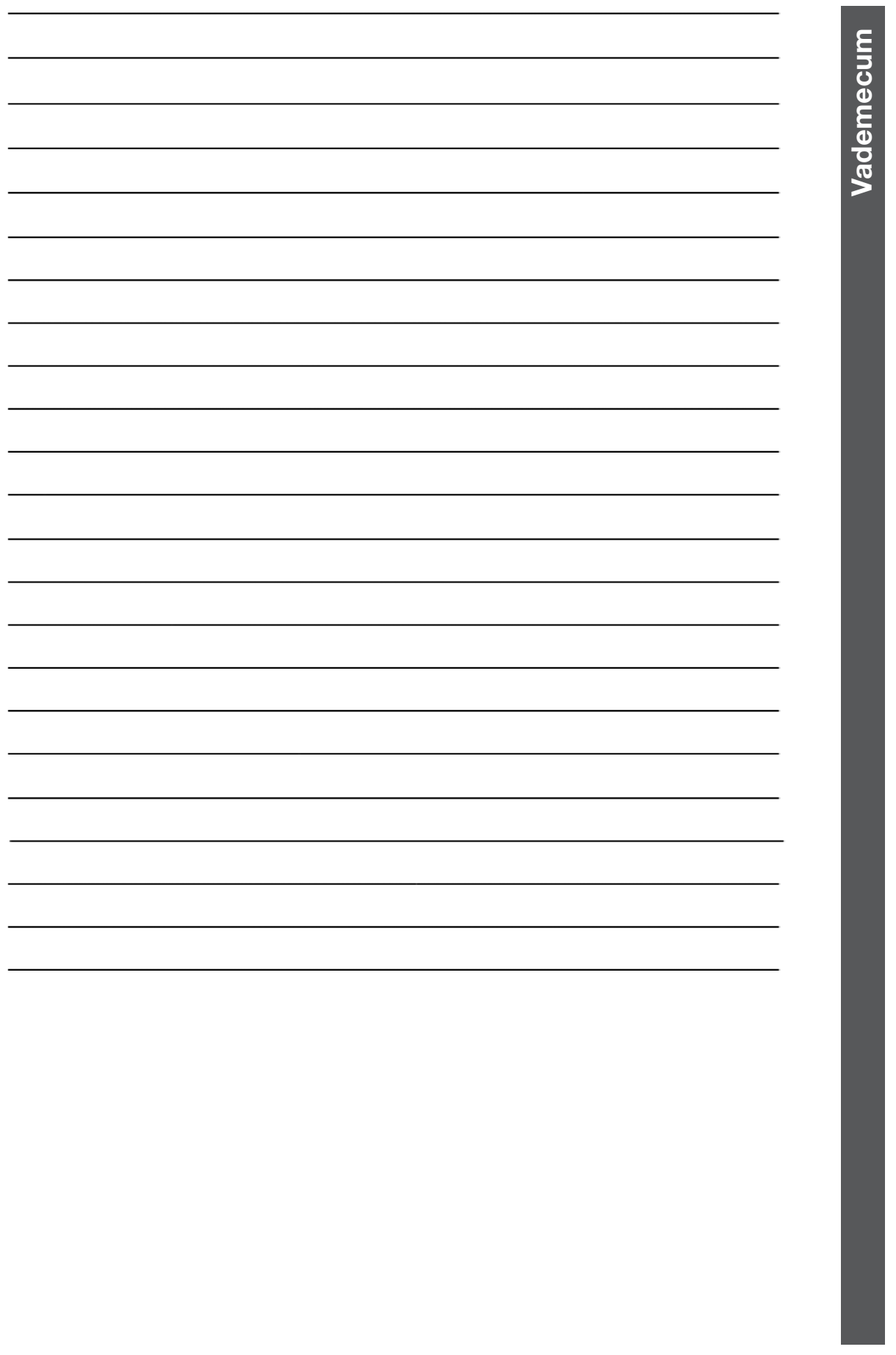


IV. DOPO L'ESPERIENZA

10. Alla fine dell'esperienza, quale valutazione posso esprimere?

Sono soddisfatto dell'esperienza che ho compiuto? Perché?

Quali sono state le criticità?

11. Che cosa può fare un tutor, secondo me?

12. Le proposte che vorrei fare per sostenere l'integrazione delle ragazze e dei ragazzi stranieri 


\title{
Allegato 10 \\ Questionario sulla valutazione dell'esperienza di tutorag- gio da parte dei ragazzi accompagnati
}

\author{
Alunno \\ Nome e cognome:
}

Classe:

Tipo di laboratorio frequentato:

$\square$ Laboratorio per l'esame di terza media

$\square$ Laboratorio di aiuto allo studio nella scuola superiore

1. Come hai saputo del laboratorio? Chi ti ha informato?

2. II calendario e gli orari andavano bene per te?

$\square$ MOLTO $\quad \square$ SI $\square$ NO $\square$ PER NIENTE

3. II laboratorio ti è stato utile?

$\square$ MOLTO $\square$ SI $\square$ NO $\square$ PER NIENTE

4. Hai imparato a studiare meglio?
$\square$ MOLTO
$\square \mathrm{SI}$
$\square \mathrm{NO}$
PER NIENTE

5. II tutor ti ha aiutato/è stato utile?
$\square$ MOLTO
$\square \mathrm{SI}$
$\square \mathrm{NO}$
PER NIENTE

6. Hai imparato cose nuove?
$\square$ MOLTO
$\square \mathrm{SI}$
$\square \mathrm{NO}$
PER NIENTE

7. In futuro ti piacerebbe partecipare ad altri laboratori come questo?
$\square$ MOLTO
$\square \mathrm{S}$
$\square \mathrm{NO}$
$\square$ PER NIENTE

8. Lo consiglieresti a un tuo amico?

Che cosa gli diresti per presentare il laboratorio?

Vuoi dirci altre cose su questa esperienza? 


\section{VAI SU: WWW.francoangeli.it}

\section{PER SCARICARE (GRATUITAMENTE) I CATALOGHI DELLE NOSTRE PUBBLICAZIONI DIVISI PER ARGOMENTI E CENTINAIA DI VOCI: PER FACILITARE LE TUE RICERCHE.}
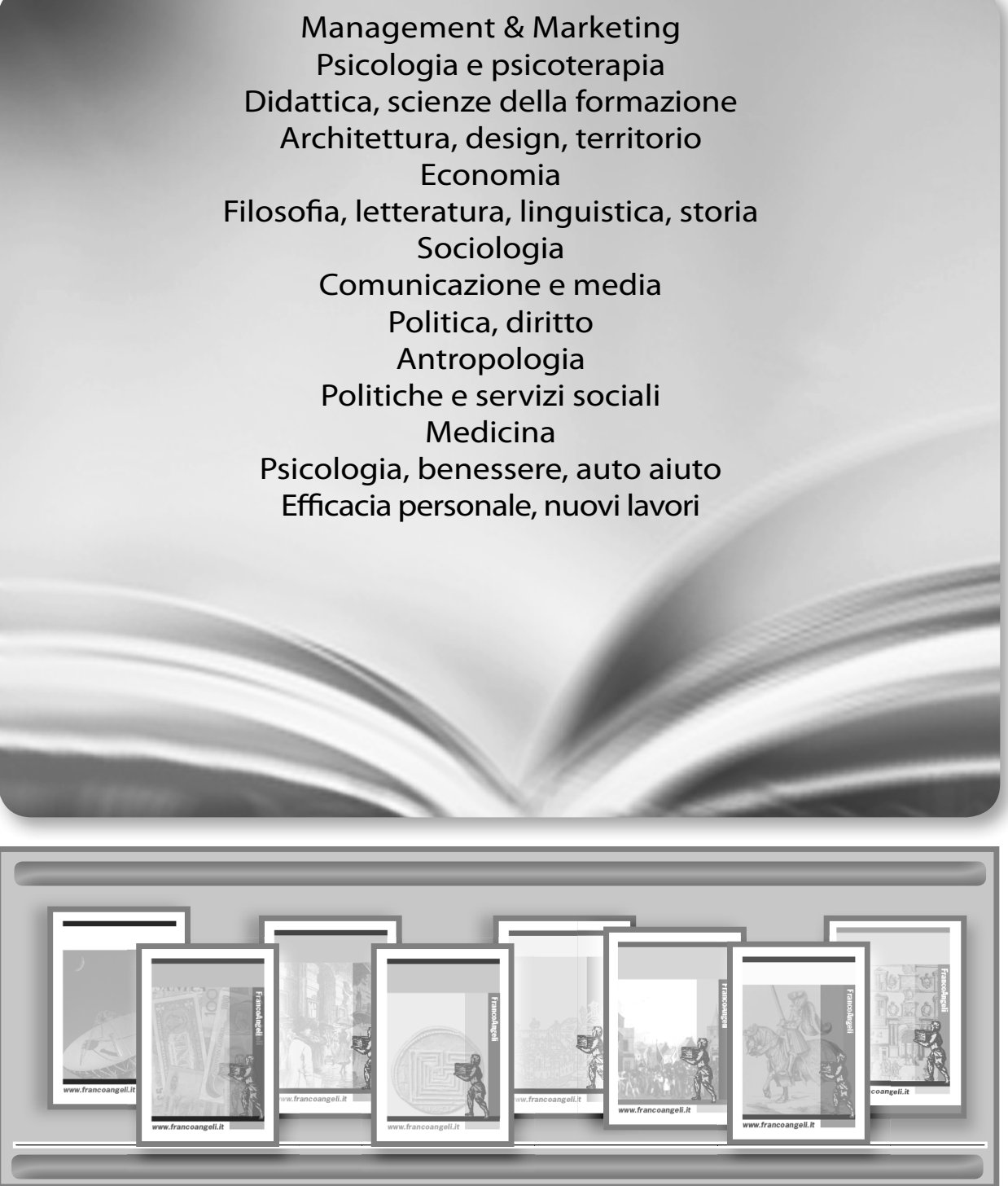


\section{ALMENO UNA STELLA}

Il titolo del libro prende a prestito l'espressione e l'auspicio che Boris Cyrulnik utilizza a proposito di resilienza dei minori che hanno vissuto una situazione di trauma o di frattura nella loro storia. Così egli scrive: "affinché il bambino o l'adolescente possano intraprendere un cammino di resilienza è necessario che accanto a loro ci sia almeno una stella, e cioè figure, rapporti e relazioni che rappresentino dei veri e propri "tutori di resilienza"".

Abbiamo individuato le possibili "stelle" sul cammino di integrazione degli adolescenti immigrati nella figura dei tutor. Si tratta di giovani, per lo più universitari, molti dei quali di origine straniera e quindi con un vissuto di migrazione comune alle ragazze e ai ragazzi accompagnati. Rappresentano delle figure di prossimità per vari aspetti: la vicinanza anagrafica e di età, le esperienze di vita molto simili, le lingue e i linguaggi condivisi. Sono vicini pur senza essere "pari": né compagni di classe, né adulti, ma collocati in quello spazio della giusta distanza che li rende prossimi come fratelli e sorelle maggiori.

Il dispositivo di tutoraggio o di mentoring è largamente diffuso e da tempo in contesti europei ed extraeuropei e sperimentato con esiti positivi come forma di aiuto e sostegno scolastico, mediazione e rispecchiamento, identificazione positiva e ri-motivazione. In Italia invece non è molto praticato, quantomeno in maniera ordinaria, continuativa e monitorata.

Il progetto "Almeno una stella" ha organizzato una rete di accoglienza e di accompagnamento per gli adolescenti neoarrivati, prevedendo azioni dedicate e di qualità per l'apprendimento della lingua e l'aiuto allo studio, per sostenere il momento dell'orientamento e la fase di passaggio dalla scuola secondaria di primo a quella di secondo grado, per far conoscere e abitare la città e i suoi luoghi di aggregazione. $\grave{E}$ stato sperimentato per due anni in sei diversi territori: a Milano (città capofila), Torino, Bologna, Arezzo, nella provincia di Trento, in Friuli. Il tutoraggio si è rivelato fortemente generativo e fecondo e ha prodotto cambiamenti importanti e profondi, sia in coloro che sono stati accompagnati, sia in chi ha accompagnato. Il testo racconta il progetto e le sue declinazioni locali; traccia il ritratto degli adolescenti stranieri, $i$ loro aspetti di vulnerabilità e di riuscita; presenta i diversi stili di essere tutor. Propone inoltre un vademecum operativo per coloro che vorranno sperimentare il dispositivo del tutoraggio a scuola e nella città. 